\title{
Superderivações e superhomomorfismos de Jordan e identidades funcionais
}

\author{
Willian Ribeiro Valencia da Silva \\ DisSERTAÇÃO APRESENTADA \\ AO \\ Instituto de Matemática e Estatística \\ DA \\ Universidade De SÃo Paulo \\ PARA \\ OBTENÇÃO DO TÍTULO \\ DE \\ Mestre em MatemáticA \\ Programa: Matemática \\ Orientador: Prof. Dr. Rodrigo Lucas Rodrigues
}

Durante o desenvolvimento deste trabalho o autor recebeu auxílio financeiro do CNPq.

São Paulo, Agosto de 2015. 


\section{Superderivações e superhomomorfismos de Jordan e identidades funcionais}

Esta versão da dissertação contém as correções e alterações sugeridas pela Comissão Julgadora durante a defesa da versão original do trabalho, realizada em 12/08/2015. Uma cópia da versão original está disponível no

Instituto de Matemática e Estatística da Universidade de São Paulo.

Comissão Julgadora:

- Prof. Dr. Rodrigo Lucas Rodrigues - Centro de Ciências-UFC

- Prof. Dr. Henrique Guzzo Junior - IME-USP

- Prof. Dr. Plamen Koshlukov - IMECC-UNICAMP 


\section{Agradecimentos}

Agradeço a Deus, pela oportunidade de concluir essa dissertação e obter o título de Mestre em Matemática. A minha mãe Cleonice e a minha irmã Cintia, por todo amor e apoio, e a toda minha família pelo incentivo. Ao meu orientador Rodrigo, pela paciência infinita e pelos bondosos conselhos. Aos meus amigos, que ao longo desses dois anos fizeram parte da minha vida, compartilhando ideias e me tornando uma pessoa melhor. Agradeço também à banca examinadora, e ao CNPq, pelo apoio financeiro. 


\section{Resumo}

SILVA, W. R. V. Superderivações e superhomomorfismos de Jordan e identidades funcionais. Dissertação (Mestrado) - Instituto de Matemática e Estatística, Universidade de São Paulo, São Paulo, 2015.

O objetivo dessa dissertação é apresentar a generalização de alguns resultados, válidos para anéis, para o contexto de superálgebras. Em 1957, I. N. Herstein provou, em [16], que toda derivação de Jordan em um anel primo de característica diferente de 2 é uma derivação. Em 1988, M. Brešar demonstrou, em [7], que este fato também é válido no caso em que o anel é semiprimo. Nos Capítulos 2 e 3, apresentamos generalizações desses resultados, dadas por M. Fošner, em 2003, em [10] e [11], e que afirmam que em uma superálgebra associativa prima, cuja parte par é não comutativa, toda superderivação de Jordan é uma superderivação, e que se $\mathcal{A}=\mathcal{A}_{0} \oplus \mathcal{A}_{1}$ é uma superálgebra associativa semiprima e $D=D_{0}+D_{1}$ é uma superderivação de Jordan, então, existem ideais graduados $U$ e $V$ de $\mathcal{A}$ tais que $D_{i}(u x)=D_{i}(u) x+(-1)^{i|u|} u D_{i}(x), i=0,1$, para todos $u \in U$ e $x \in \mathcal{A}$, e $\left[v_{0}, x_{0}\right]=0$, para quaisquer $v_{0} \in V_{0}$ e $x_{0} \in \mathcal{A}_{0}$. Além disso, $U \cap V=0$ e $U \oplus V$ é um ideal essencial de $\mathcal{A}$, isto é, a interseção de $U \oplus V$ com qualquer ideal graduado não nulo de $\mathcal{A}$ é não nula. Se $U=0$, então, $\mathcal{A}_{0}$ é comutativa e se $V=0$, então, $D$ é uma superderivação. Em 1956, I. N. Herstein mostrou, em [15], que todo homomorfismo de Jordan sobrejetor, de um anel qualquer em um anel primo de característica diferente de 2 e 3 , é um homomorfismo ou um antihomomorfismo, e em 1957, M. Smiley provou, em [31], o mesmo resultado sem usar a hipótese de que a característica do anel é diferente de 3. No Capítulo 4, apresentamos a generalização desse resultado dada por K. Beidar, M. Brešar e M. Chebotar, em 2003, em [3], e que afirma que todo superhomomorfismo de Jordan sobrejetor de uma superálgebra associativa qualquer em uma superálgebra associativa prima, cuja parte par não é comutativa, é um superhomomorfismo ou um superantihomomorfismo. No Capítulo 5, introduzimos o resultado de W. Baxter e W. Martindale, 3º, de 1979, dado em [25], que afirma que se $\varphi$ é um homomorfismo de Jordan sobrejetor de um anel $T$ em um anel semiprimo $R$, de característica diferente de 2, então, existe um ideal essencial $E$ de $T$ tal que a restrição de $\varphi$ a $E$ é a "soma direta" de um homomorfismo com um antihomomorfismo. Finalmente, no último capítulo, fazemos uma exposição da teoria de identidades funcionais dada por M. Brešar, M. Chebotar e W. Martindale, $3^{\circ}$, em [8], apresentamos a generalização da teoria para superálgebras dada por Yu Wang [32], em 2011, e ainda um resultado de Yu Wang e Yao Wang [33], de 2014, que afirma que se $\alpha$ é um superhomomorfismo de Jordan de uma superálgebra $\mathcal{A}$ em uma superálgebra unitária $\mathcal{Q}$, tal que $\alpha(\mathcal{A})$ é um subconjunto 4-superlivre de $\mathcal{Q}$, então, $\alpha$ é uma "soma direta" de um superhomomorfismo com um superantihomomorfismo. Finalmente, apresentamos uma contribuição original para a classificação das superderivações de Jordan de grau 0.

Palavras-chave: superderivações, superhomomorfismos, superálgebras, identidades funcionais. 


\section{Abstract}

\section{SILVA, W. R. V. Jordan superderivations and superhomomorphisms and functional iden-}

tities. Dissertação (Mestrado) - Instituto de Matemática e Estatística, Universidade de São Paulo, São Paulo, 2015.

The goal of this dissertation is to present the generalization of some results, which hold in rings, for the context of superalgebras. In 1957, I. N. Herstein proved, in [16], that every Jordan derivation on a prime ring with characteristic not 2 is a derivation. In 1988, M. Brešar proved in [7] that the result still holds when the ring is semiprime. In the Chapters 2 and 3, we present generalizations for these results, given by M. Fošner, in 2003, in [10] and [11], which state that on a prime associative superalgebra, whose even part is noncommutative, every Jordan superderivation is a superderivation, and if $\mathcal{A}=\mathcal{A}_{0} \oplus \mathcal{A}_{1}$ is a semiprime associative superalgebra and $D=D_{0}+D_{1}$ is a Jordan superderivation, then there exist graded ideals $U$ and $V$ of $\mathcal{A}$ such that $D_{i}(u x)=$ $D_{i}(u) x+(-1)^{i|u|} u D_{i}(x), i=0,1$, for all $u \in U$ and $x \in \mathcal{A}$, and $\left[v_{0}, x_{0}\right]=0$, for all $v_{0} \in V_{0}$ and $x_{0} \in \mathcal{A}_{0}$. Moreover, $U \cap V=0$ and $U \oplus V$ is an essential ideal of $\mathcal{A}$, that is, the intersection of $U \oplus V$ and any nonzero graded ideal of $\mathcal{A}$ is nonzero. If $U=0$, then $\mathcal{A}_{0}$ is commutative and if $V=0$, then $D$ is a superderivation. In 1956, I. N. Herstein proved in [15] that every Jordan homomorphism onto a prime ring with characteristic not 2 or 3 , is either a homomorphism or an antihomomorphism, and in 1957, M. Smiley proved, in [31], the same result without assuming that the characteristic of the ring is not 3. In the Chapter 4, we present a generalization of this result given by K. Beidar, M. Brešar and M. Chebotar, in 2003, in [3], which states that every Jordan superhomomorphism from an associative superalgebra onto a prime associative superalgebra, whose even part is noncommutative, is either a superhomomorphism or a superantihomomorphism. In Chapter 5, we present a result given by W. Baxter and W. Martindale, 3rd, in 1979, in [25], which states the if $\varphi$ is a Jordan homomorphism from a ring $T$ onto a semiprime ring $R$, with characteristic not 2 , then there exists an essential ideal $E$ of $T$ such that the restriction of $\varphi$ to $E$ is a "direct sum" of a homomorphism and an antihomomorphism. Finally, in the last chapter, we present the theory of functional identities given by M. Brešar, M. Chebotar and W. Martindale, 3rd, in [8], we also present the generalization of the theory for superalgebras given by Yu Wang [32], in 2011, and a result given by Yu Wang and Yao Wang [33], in 2014, which states that if $\alpha$ is a Jordan superhomomorphism from a superalgebra $\mathcal{A}$ into a unital superalgebra $\mathcal{Q}$, such that $\alpha(\mathcal{A})$ is a 4 -superfree subset of $\mathcal{Q}$, then $\alpha$ is a "direct sum" of a superhomomorphism and superantihomomorphism. Finally, we present an original contribution to the classification of the Jordan superderivations of degree 0 .

Keywords: superderivations, superhomomorphisms, superalgebras, functional identities. 


\section{Sumário}

$\begin{array}{ll}\text { Introdução } & 2\end{array}$

1 Conceitos Preliminares $\quad 3$

1.1 Anéis e ideais primos e semiprimos . . . . . . . . . . . . . . 3

1.2 Superálgebras primas, semiprimas e ideais graduados . . . . . . . . . . . . . 10

1.3 Álgebra de Grassmann, superidentidades e superálgebras de Jordan . . . . . . . . . . 18

1.4 Adjunção de identidades . . . . . . . . . . . . . . . . . . . . . . . 20

2 Derivações e superderivações de Jordan em anéis e superálgebras primas $\quad 21$

2.1 Derivações de Jordan em anéis primos . . . . . . . . . . . . . . . . . 21

2.2 Superderivações de Jordan em superálgebras primas . . . . . . . . . . . . . . . . . 24

2.2.1 Superderivações de Jordan de grau $0 \ldots \ldots$. . . . . . . . . . . . . . . 32

2.2 .2 Superderivações de Jordan de grau $1 \ldots \ldots$. . . . . . . . . . . . . . 36

3 Derivações e superderivações de Jordan em anéis e superálgebras semiprimas 43

3.1 Derivações de Jordan em anéis semiprimos . . . . . . . . . . . . . . . . . . 43

3.2 Superderivações de Jordan em superálgebras semiprimas . . . . . . . . . . . . . . 45

3.2 .1 Superderivações de Jordan de grau $0 \ldots \ldots$. . . . . . . . . . 50

3.2 .2 Superderivações de Jordan de grau $1 \ldots \ldots \ldots$. . . . . . . . . . 54

4 Homomorfismos e superhomomorfismos de Jordan em anéis e superálgebras primas $\quad 65$

4.1 Homomorfismos de Jordan sobre anéis primos . . . . . . . . . . . . . . . . . 65

4.2 Superhomomorfismos de Jordan sobre superálgebras primas . . . . . . . . . . . . . 70

5 Homomorfismos de Jordan em anéis semiprimos $\quad 81$

6 Identidades funcionais e superhomomorfismos de Jordan $\quad 91$

6.1 A teoria de identidades funcionais em anéis . . . . . . . . . . . . . . . . 91

6.2 A teoria de identidades funcionais em superálgebras . . . . . . . . . . . . . . . . 115

6.3 Superhomomorfismos de Jordan em superálgebras . . . . . . . . . . . . . . . . . . 119

$\begin{array}{ll}\text { Referências Bibliográficas } & 133\end{array}$ 


\section{Introdução}

Na física de partículas existe o chamado Modelo Padrão que incorpora tudo o que sabemos até então do mundo material: as partículas de matéria (os férmions, como elétrons, prótons e nêutrons) e as partículas que transmitem a força entre as partículas de matéria (os bósons, como o fóton, no caso do eletromagnetismo e o glúon, na força fraca). Alguns teóricos apresentaram a Teoria de Supersimetria nos anos 70 para ligar esses dois tipos básicos de partículas. A supersimetria interrelaciona portanto partículas completamente diferentes: num conjunto de partículas se os estados quânticos de dois férmions idênticos (por exemplo dois elétrons) são invertidos, o estado quântico total do conjunto se inverte, a inversão dos estados de dois bósons idênticos, por outro lado, não altera o estado; essa característica faz com que férmions jamais ocupem o mesmo estado quântico enquanto que os bósons unem-se prontamente em estados idênticos. Nenhuma simetria simples existente na física possui essa capacidade, já que essas relacionam necessariamente bósons com bósons e férmions com férmions. As simetrias simples são descritas matematicamente pelos grupos e pelas álgebras de Lie, porém elas não são capazes de cancelar ou criar a inversão que ocorre quando os férmions são invertidos, sendo impotentes em "transformar" férmions em bósons ou vice-versa. As transformações supersimétricas atribuem a cada bóson conhecido um "superparceiro" fermiônico e a cada férmion um "superparceiro" bosônico, o que é suficiente para a conversão entre férmions e bósons, a Teoria de Supersimetria utiliza dessa maneira a Teoria das Superálgebras, ou álgebras $\mathbb{Z}_{2^{-}}$ graduadas. As superálgebras e as suas representações fornecem assim uma estrutura algébrica para a formulação de supersimetria. Essa teoria também representa um papel importante relacionado à supergeometria, onde são introduzidos conceitos como os de variedades graduadas, supervariedades e superesquemas. Nas últimas décadas a teoria de superálgebras tem sido um dos campos mais ativos e férteis em álgebra, muitos resultados sobre esse tema têm sido descobertos por autores como V. G. Kac, C. Gómez-Ambrosi, C. T. C. Wall, I. P. Shestakov e outros (por exemplo em [12], [13], [14], [22], [23], [28], [29], [30]). Uma superálgebra $\mathcal{A}$ é uma álgebra $\mathbb{Z}_{2}$-graduada, isto é, existem submódulos $\mathcal{A}_{0}$ e $\mathcal{A}_{1}$ de $\mathcal{A}$ tais que $\mathcal{A}=\mathcal{A}_{0} \oplus \mathcal{A}_{1}$ e $\mathcal{A}_{0} \mathcal{A}_{0} \subseteq \mathcal{A}_{0}$ (e portanto $\mathcal{A}_{0}$ é uma subálgebra de $\mathcal{A}), \mathcal{A}_{0} \mathcal{A}_{1} \subseteq \mathcal{A}_{1}, \mathcal{A}_{1} \mathcal{A}_{0} \subseteq \mathcal{A}_{1}$ e $\mathcal{A}_{1} \mathcal{A}_{1} \subseteq \mathcal{A}_{0}$. Dizemos que $\mathcal{A}_{0}$ é a parte par e $\mathcal{A}_{1}$ é a parte ímpar de $\mathcal{A}$.

No contexto das álgebras não necessariamente associativas, as álgebras de Jordan, assim como as álgebras de Lie, foram as primeiras álgebras não associativas que atraíram a atenção dos matemáticos. As álgebras de Jordan foram introduzidas por Pascual Jordan, físico alemão, em 1933, e tinham por objetivo formalizar a noção de uma álgebra de observáveis. Em física, mais especificamente física quântica, um observável é uma propriedade do estado (quântico) de sistema (este estado quântico é dado por um vetor chamado vetor de estado) que pode ser determinada por alguma sequência de operações físicas (por exemplo submeter o sistema a vários campos eletromagnéticos). Observáveis que fazem sentido fisicamente devem satisfazer "leis de transformação" que relacionam observações 
feitas por diferentes observadores em diferentes referenciais. Essas leis de transformação são automorfismos. Na interpretação usual de mecânica quântica (o "Modelo de Copenhagen", desenvolvido em 1927 por Niels Bohr e Werner Heisenberg, enquanto trabalhavam juntos em Copenhague) os observáveis físicos são representados por matrizes hermitianas. As operações básicas de matrizes são a multiplicação por um escalar complexo, a adição, a multiplicação entre matrizes e a construção da matriz transposta conjugada complexa (operador adjunto), mas essas operações não são "observáveis", no sentido de que não necessariamente produzem novas matrizes hermitianas (por exemplo a multiplicação por escalar só produz uma matriz hermitiana se o escalar for real). Assim as álgebras de Jordan surgem da busca de um novo conjunto algébrico para a mecânica quântica. Pascual Jordan desejava estudar as propriedades intrínsecas das matrizes hermitianas, a fim de transcrevê-las em propriedades algébricas formais e ver quais outros possíveis sistemas não-matriciais satisfaziam esses axiomas. Depois de certa experimentação empírica, Jordan decidiu que, apesar de existirem diversas formas de se combinar duas matrizes hermitianas para se obter outra, todas elas poderiam ser expressas em termos da "quasi-multiplicação" $x \circ y=\frac{1}{2}(x y+y x)$. O próximo passo da investigação empírica das propriedades algébricas das matrizes hermitianas, era decidir quais axiomas ou leis as operações sobre matrizes desse tipo satisfaziam, e Jordan pensou que a lei chave que governava a quasi-multiplicação, além da óbvia comutatividade, era $x^{2} \circ(y \circ x)=\left(x^{2} \circ y\right) \circ x$, a qual chamamos de identidade de Jordan. Assim define-se álgebra de Jordan como sendo uma álgebra que é comutativa e satisfaz a identidade de Jordan (com a própria operação da álgebra). A teoria das álgebras de Jordan tem sido uma área de pesquisa de destaque, com aplicações em diversas áreas, entre elas as álgebras de Lie, geometria diferencial, geometria projetiva, genética, equações diferenciais, probabilidade e estatística.

A teoria de identidades funcionais é relativamente nova, ela surgiu da tese de doutorado de Matej Brešar, em 1990, a qual foi acompanhada nos anos seguintes por uma série de artigos nos quais ele estudou algumas identidades funcionais básicas. A figura central no estabelecimento dos fundamentos da teoria geral foi Konstantin I. Beidar (1951-2004). A principal motivação para o desenvolvimento desta teoria foi a procura de ferramentas capazes de responder as conjecturas de Herstein a repeito dos homomorfismos de Lie e derivações de Lie em anéis associativos, das quais foram obtidas soluções completas, utilizando-se de praticamente toda a teoria avançada de identidades funcionais. Contudo, essa teoria encontra conexões em diferentes áreas, por exemplo em álgebras de Lie, de Jordan e em outras álgebras não associativas, em álgebra linear, na teoria de operadores, em análise funcional e em física-matemática. Uma identidade funcional pode ser informalmente descrita como uma identidade envolvendo elementos quaisquer de um anel e funções que são, a priori, "desconhecidas", isto é, os elementos são multiplicados por valores de funções. O objetivo da teoria geral de identidades funcionais é determinar a forma dessas funções, ou, quando isto não é possível, determinar a estrutura do anel admitindo a identidade funcional em questão.

Uma parte considerável dos teoremas de I. N. Herstein provados para anéis (álgebras) foi recentemente estendida para superálgebras. Nessa dissertação apresentamos algumas generalizações dessas e de outros autores, que se referem aos conceitos de homomorfismos e derivações de Jordan, e seus conceitos generalizadores de superhomomorfismos e superderivações de Jordan. Além disso, fazemos no último capítulo uma exposição da recente Teoria de Identidades Funcionais, conectando ainda essa teoria aos problemas tratados anteriormente na dissertação, e apresentamos uma contribuição original para a classificação das superderivações de Jordan de grau 0. 


\section{Capítulo 1}

\section{Conceitos Preliminares}

Neste capítulo apresentamos e discutimos propriedades dos conceitos a serem utilizados ao longo da dissertação. Também enunciamos e provamos alguns resultados necessários nas demonstrações dos capítulos seguintes. Dividimos esses conceitos em quatro seções.

\subsection{Anéis e ideais primos e semiprimos}

Ao longo da dissertação consideraremos que todos os anéis são associativos, caso contrário diremos explicitamente.

Definição 1.1.1. Um anel $A$ é dito primo se para quaisquer ideais não nulos $I, J$ de $A$ temos que o seu produto $I J$ é não nulo.

Exemplo 1.1.1. O anel dos inteiros $\mathbb{Z}$ é primo, pois se $n \mathbb{Z}$ e $m \mathbb{Z}$ são ideais não nulos de $\mathbb{Z}$, então, $(n \mathbb{Z})(m \mathbb{Z})=(n m) \mathbb{Z} \neq 0$.

Exemplo 1.1.2. Se $n \in \mathbb{Z}$ não é primo podemos escrevê-lo como $n=p q$, com $p$ e $q$ não nulos e diferentes de \pm 1 . Assim, considerando o anel das classes de congruência $\mathbb{Z}_{n}$, vemos que os ideais gerados por $\bar{p}$ e $\bar{q}$ são não nulos mas seu produto $\langle\bar{p}\rangle\langle\bar{q}\rangle=\langle\bar{n}\rangle$ é nulo. Portanto, $\mathbb{Z}_{n}$ não é um anel primo.

Definição 1.1.2. Um ideal $P$ de um anel $A$ é dito primo se dados ideais $I, J$ de $A$ tais que $I J \subseteq P$ então $I \subseteq P$ ou $J \subseteq P$.

A definição acima de ideal primo equivale a "se $I, J$ são ideais de $A$ tais que $P \subseteq I, P \subseteq J$ e $I J \subseteq P$, então, $P=I$ ou $P=J$ ". De fato, assuma a primeira definição e considere ideais $I, J$ de $A$ satisfazendo $P \subseteq I, P \subseteq J$ e $I J \subseteq P$, então, $I \subseteq P$ ou $J \subseteq P$, o que implica $I=P$ ou $J=P$. Suponha agora a segunda definição, e sejam $I, J$ ideais de $A$ tais que $I J \subseteq P$, então, $I+P$ e $J+P$ são ideais de $A$ tais que $P \subseteq I+P, P \subseteq J+P$ e $(I+P)(J+P) \subseteq P$ e, portanto, $P=I+P$ ou $P=J+P$, o que por sua vez implica $I \subseteq P$ ou $J \subseteq P$.

Além disso, claramente se $P$ é um ideal primo e temos um número finito $I_{1}, I_{2}, \ldots, I_{n}$ de ideais de $A$ tais que $I_{1} I_{2} \cdots I_{n} \subseteq P$, então, existe $j \in\{1,2, \ldots, n\}$ tal que $I_{j} \subseteq P$.

Proposição 1.1.1. Um ideal $P$ de um anel $A$ é primo se, e somente se, o anel quociente $A / P$ é um anel primo. 
Demonstração. Assuma que $P$ é um ideal primo de $A$ e sejam $U$ e $V$ ideais de $A / P$ tais que $U V=0$. Considere a projeção natural $\pi: A \rightarrow A / P$. Note $\pi^{-1}(U V)=\pi^{-1}(0)=P$, o que implica $\pi^{-1}(U) \pi^{-1}(V) \subseteq \pi^{-1}(U V)=P$. Como $P$ é primo, temos $\pi^{-1}(U) \subseteq P$ ou $\pi^{-1}(V) \subseteq P$, pois $\pi^{-1}(U)$ e $\pi^{-1}(V)$ são ideais de $A$, uma vez que são imagens inversas de ideais por homomorfismo. Portanto, $U=\pi\left(\pi^{-1}(U)\right) \subseteq \pi(P)=0$ ou $V=\pi\left(\pi^{-1}(V)\right) \subseteq \pi(P)=0$, pois $\pi$ é sobrejetora.

Reciprocamente, suponha que $A / P$ é um anel primo. Se $I$ e $J$ são ideais de $A$ tais que $I J \subseteq P$, então $\pi(I) \pi(J)=\pi(I J) \subseteq \pi(P)=0$. Assim, como $\pi(I)$ e $\pi(J)$ são ideais de $A / P$, pois $\pi$ é sobrejetora, podemos concluir que vale $\pi(I)=0$ ou $\pi(J)=0$, isto é, $I \subseteq \pi^{-1}(0)=P$ ou $J \subseteq$ $\pi^{-1}(0)=P$.

Teorema 1.1.1. Se $P$ é um ideal de um anel $A$, então, as seguintes condições são equivalentes:

(i) $P$ é um ideal primo;

(ii) se $a, b \in A$ são tais que $a A b \subseteq P$, então, $a \in P$ ou $b \in P$;

(iii) se $(a)$ e $(b)$ são ideais principais de $A$ tais que $(a)(b) \subseteq P$, então, $a \in P$ ou $b \in P$;

(iv) se $U$ e $V$ são ideais à direita de $A$ tais que $U V \subseteq P$, então, $U \subseteq P$ ou $V \subseteq P$;

(v) se $U$ e $V$ são ideais à esquerda de $A$ tais que $U V \subseteq P$, então, $U \subseteq P$ ou $V \subseteq P$.

Demonstração. (i) $\Rightarrow$ (ii) Se $a, b \in A$ são tais que $a A b \subseteq P$, segue que as inclusões $A a A b A \subseteq P$ e $(A a A)(A b A) \subseteq P$ são válidas. Desse modo, como $A a A$ e $A b A$ são ideais de $A$ temos $A a A \subseteq P$ ou $A b A \subseteq P$. Agora, se $I=(a)$ e $J=(b)$ concluímos $I^{3} \subseteq A a A \subseteq P$ ou $J^{3} \subseteq A b A \subseteq P$, o que implica $I \subseteq P$ ou $J \subseteq P$, e portanto, $a \in P$ ou $b \in P$.

(ii) $\Rightarrow$ (iii) Suponha que $(a)$ e $(b)$ sejam ideais principais tais que $(a)(b) \subseteq P$, então, $a A b \subseteq(a)(b) \subseteq$ $P$. Logo, $a \in P$ ou $b \in P$.

(iii) $\Rightarrow$ (iv) Suponha que $U$ e $V$ sejam ideais à direita satisfazendo $U V \subseteq P$. Se $U \nsubseteq P$, então existe $u \in U$ tal que $u \notin P$. Para cada $v \in V$, temos $(u)(v) \subseteq U V+A U V \subseteq P$ o que, pela hipótese, implica $v \in P$, pois $u \notin P$. Consequentemente, $V \subseteq P$. Portanto, $U \subseteq P$ ou $V \subseteq P$.

(iii) $\Rightarrow$ (v) Sejam $U$ e $V$ ideais à esquerda de $A$ tais que $U V \subseteq P$. Se $U \nsubseteq P$, então, existe um elemento $u \in U$ tal que $u \notin P$. Desse modo dado $v \in V$, temos $(u)(v) \subseteq U V+U V A \subseteq P, \mathrm{o}$ que implica $v \in P$, pela hipótese, isto é, $V \subseteq P$. Portanto, $U \subseteq P$ ou $V \subseteq P$.

Finalmente, assumindo (iv) ou (v), temos que se $I$ e $J$ são ideais de $A$ tais que $I J \subseteq P$, então, em particular, $I$ e $J$ são ideais à direita e à esquerda e portanto, $I \subseteq P$ ou $J \subseteq P$. Logo, $P$ é um ideal primo.

Proposição 1.1.2. Um anel $A$ é primo se, e somente se, satisfaz a seguinte propriedade:

$$
\text { "se } a, b \in A \text { são tais que } a A b=0 \text {, então, } a=0 \text { ou } b=0 \text { ". }
$$

Demonstração. Um anel $A$ é primo se, e somente se, $A / 0 \cong A$ é anel primo, se, e somente se, 0 é ideal primo. Pelo item (ii) do Teorema 1.1.1, sabemos que isso equivale à propriedade (1.1).

Proposição 1.1.3. Seja $A$ um anel primo de característica diferente de 2. Se $a, b \in A$ são tais que $a r b+b r a=0$, para todo $r \in A$, então, $a=0$ ou $b=0$. 
Demonstração. Suponha $a r b+b r a=0$, para todo $r \in A$. Em particular, para $r=s a t$, onde $s$ e $t$ são quaisquer elementos de $A$, temos $a s a t b+b s a t a=0$. Além disso, $a s b+b s a=0$ implica $b s a=-a s b$. Analogamente, também vale $a t b=-b t a$. Fazendo essas substituições, obtemos $-a s b t a-a s b t a=0$, isto é, 2asbta $=0$. Logo, asbta $=0$, pois $A$ tem característica diferente de 2, ou seja, $a A(b t a)=0$. Desse modo, pela Proposição 1.1.2, segue $a=0$ ou bta $=0$. Entretanto, no segundo caso, como $t$ é um elemento qualquer, temos $b A a=0$, o que implica $b=0$ ou $a=0$.

Dado um anel $A$, definimos $[x, y]=x y-y x$ o comutador de quaisquer dois elementos $x, y \in A$. A seguir, apresentamos duas identidades que serão utilizadas na demonstração do próximo lema.

1. Identidade de Semi-Jacobi: $[x, y z]=y[x, z]+[x, y] z$;

$$
\begin{aligned}
{[x, y z] } & =x y z-y z x \\
& =y x z-y z x+x y z-y x z \\
& =y[x, z]+[x, y] z .
\end{aligned}
$$

2. Identidade de Jacobi: $[x,[y, z]]=[y,[x, z]]+[z,[y, x]]$.

$$
\begin{aligned}
{[y,[x, z]]+[z,[y, x]] } & =y x z-y z x-x z y+z x y+z y x-z x y-y x z+x y z \\
& =x y z-x z y-y z x+z y x \\
& =[x,[y, z]] .
\end{aligned}
$$

Lema 1.1.1. Se $A$ é um anel primo tal que

$$
[a, b]^{2}=0
$$

para todos $a, b \in A$, então, $A$ é comutativo, e portanto, não possui divisores de zero não nulos.

Demonstração. Linearizando (1.2) em $b$ obtemos $[a, b][a, c]+[a, c][a, b]=0$, isto é, $[a, b][a, c]=$ $-[a, c][a, b]$, para quaisquer $a, b, c \in A$. Usando esta igualdade e (1.2), temos

$$
[a, b][a, d[c, b]][a, b]=-[a, d[c, b]][a, b][a, b]=-[a, d[c, b]][a, b]^{2}=0
$$

Agora, aplicando a identidade de Semi-Jacobi, obtemos

$$
\begin{aligned}
0 & =[a, b][a, d[c, b]][a, b] \\
& =[a, b](d[a,[c, b]]+[a, d][c, b])[a, b] \\
& =[a, b] d[a,[c, b]][a, b]+[a, b][a, d][c, b][a, b]
\end{aligned}
$$

Além disso, $[a, b][a, d][c, b][a, b]=-[a, d][a, b][c, b][a, b]=[a, d][c, b][a, b]^{2}=0$, o que implica

$$
[a, b] d[a,[c, b]][a, b]=0, \quad \text { para todo } d \in A,
$$

ou seja, $[a, b] A([a,[c, b]][a, b])=0$. Como $A$ é um anel primo, podemos concluir $[a, b]=0$ ou $[a,[c, b]][a, b]=0$. Assumindo que ocorre a segunda igualdade, trocando $a$ por $b$ temos $[b,[c, a]][b, a]=$ 
0 e portanto, $[b,[a, c]][a, b]=0$. Desse modo, pela identidade de Jacobi, segue

$$
[c,[a, b]][a, b]+[a,[b, c]][a, b]=0 .
$$

Entretanto, $[a,[b, c]][a, b]=-[a,[c, b]][a, b]=0$, e então, $[c,[a, b]][a, b]=0$, ou seja, $0=c[a, b]^{2}-$ $[a, b] c[a, b]=-[a, b] c[a, b]$, para todo $c \in A$, isto é, $[a, b] A[a, b]=0$, e novamente concluímos $[a, b]=$ 0 . Portanto $[a, b]=0$, para todos $a, b \in A$, ou seja, $A$ é comutativo. Agora, se $a \in A$ é tal que existe $0 \neq b \in A$ tal que $a b=0$, então, como $A$ é comutativo, temos $a x b=a b x=0$, para todo $x \in A$, isto é, $a A b=0$, o que implica $a=0$, pois $b \neq 0$.

Definição 1.1.3. Um subconjunto $M$ de $A$ é um m-sistema se satisfaz a seguinte propriedade:

"se $a, b \in M$, então, existe $x \in A$ tal que $a x b \in M$ ".

Lema 1.1.2. Um ideal $P$ de um anel $A$ é primo se, e somente se, $C(P)$, o complementar de $P$ em $A$, é um m-sistema.

Demonstração. Assuma que $P$ é um ideal primo, então, pelo Teorema 1.1.1, temos que se $a, b \in A$ são elementos tais que $a A b \subseteq P$, então, $a \in P$ ou $b \in P$, e a recíproca também é válida. Sejam $a, b \in C(P)$, isto é, $a, b \notin P$ e portanto, $a A b \nsubseteq P$. Assim, existe $x \in A$ tal que $a x b \notin P$, ou seja, $a x b \in C(P)$. Reciprocamente, assuma que $C(P)$ é um m-sistema e sejam $a, b \in A$ tais que $a A b \subseteq P$. Se $a \notin P$ e $b \notin P$, então, existe $x \in A$ tal que $a x b \notin P$, o que é uma contradição. Portanto, $a \in P$ ou $b \in P$ e podemos concluir que $P$ é primo.

Definição 1.1.4. O radical primo, $\mathcal{B}(I)$, de um ideal $I$ de um anel $A$ é o conjunto

$$
\mathcal{B}(I)=\{a \in A \mid \text { se } M \text { é um m-sistema de } A \text { tal que } a \in M \text {, então, } M \cap I \neq \emptyset\} .
$$

Segue, dessa definição, que $I$ e $\mathcal{B}(I)$ estão contidos nos mesmos ideais primos. Claramente, se $P$ é um ideal primo de $A$ que contém $\mathcal{B}(I)$, então, $I \subseteq P$, pois $I \subseteq \mathcal{B}(I)$. Agora, sejam $P$ um ideal primo de $A$ que contém $I$ e $a \in \mathcal{B}(I)$. Se $a \notin P$, então, $a \in C(P)$, o qual é um m-sistema, e portanto $C(P) \cap I \neq \emptyset$, pela definição de $\mathcal{B}(I)$, o que é uma contradição, pois $I \subseteq P$. Logo, $a \in P$ e $\mathcal{B}(I) \subseteq P$. Além disso, para um elemento qualquer $a \in A$, o conjunto $D=\left\{a^{i} \mid i=1,2,3, \ldots\right\}$ é fechado pela multiplicação e assim, é um m-sistema (note que se $a^{i}, a^{j} \in D$, então, $a \in A$ é tal que $\left.a^{i} a a^{j}=a^{i+j+1} \in D\right)$. Desse modo, se $a \in \mathcal{B}(I)$, então, existe um inteiro positivo $n$ tal que $a^{n} \in I$. Com essas observações podemos provar mais alguns resultados.

Teorema 1.1.2. Se $I$ é um ideal de um anel $A$, então, o radical primo de $I, \mathcal{B}(I)$, coincide com a interseção de todos os ideais primos de $A$ que contêm $I$.

Demonstração. Seja $\left\{P_{\alpha} \mid \alpha \in \Lambda\right\}$ o conjunto de todos os ideais primos de $A$ que contêm $I$. Pelas observações anteriores, temos $\mathcal{B}(I) \subseteq \bigcap_{\alpha} P_{\alpha}$. Agora, suponha que $a \in A$ seja tal que $a \notin \mathcal{B}(I)$, então, existe um m-sistema $M$ de $A$ satisfazendo $a \in M$ e $M \cap I=\emptyset$. Consideremos o conjunto

$$
\Omega=\{K \text { ideal de } A \text { tal que } I \subseteq K \text { e } M \cap K=\emptyset\}
$$

Observe $\Omega \neq \emptyset$, pois $I \in \Omega$; e ordenando parcialmente $\Omega$ com a inclusão de conjuntos verifica-se facilmente que toda cadeia de $\Omega$ admite cota superior. Portanto, pelo Lema de Zorn, $\Omega$ possui um 
elemento maximal $P$. Claramente $a \notin P$, pois $a \in M$ e $M \cap P=\emptyset$. Vamos provar que $P$ é primo usando a contrapositiva do item (iii) do Teorema 1.1.1. Suponha que $x, y \in A$ são elementos tais que $x \notin P$ e $y \notin P$. Como $P$ é maximal em $\Omega$, e $P$ está contido propriamente nos ideais $P+(x)$ e $P+(y)$, temos $M \cap(P+(x)) \neq \emptyset \neq M \cap(P+(y))$. Assim, se $m_{1} \in M \cap(P+(x))$ e $m_{2} \in M \cap(P+(y))$, então, existe $b \in A$ tal que $m_{1} b m_{2} \in M$, e $m_{1} b m_{2} \in(P+(x))(P+(y))$, pois $M$ é um m-sistema. Se supossemos $(x)(y) \subseteq P$, teríamos $(P+(x))(P+(y)) \subseteq P$, e então, $m_{1} b m_{2} \in P$, o que seria uma contradição com o fato de que vale $M \cap P=\emptyset$. Portanto, $(x)(y) \nsubseteq P$ e $P$ é um ideal primo.

Desse modo, provamos que $P$ é um ideal primo de $A$ que contém $I$ e $a \notin P$, o que implica $a \notin \bigcap_{\alpha} P_{\alpha} . \log 0, \bigcap_{\alpha} P_{\alpha} \subseteq \mathcal{B}(I)$.

Definição 1.1.5. Um anel $\mathcal{A}$ é semiprimo se não possui ideais nilpotentes não nulos.

Exemplo 1.1.3. Todo anel primo é semiprimo.

Um anel semiprimo $A$ possui a seguinte propriedade: "existe uma família de ideais primos de $A$, $\left\{\mathcal{Q}_{\alpha} \mid \alpha \in \Lambda\right\}$ tal que $\bigcap_{\alpha} \mathcal{Q}_{\alpha}=0$ ". A fim de provar esta propriedade precisamos de alguns resultados preliminares.

Definição 1.1.6. Um ideal $\mathcal{Q}$ de um anel $A$ é semiprimo se satisfaz a seguinte propriedade:

"se $I$ é um ideal de $A$ tal que $I^{2} \subseteq \mathcal{Q}$, então, $I \subseteq \mathcal{Q}$ ".

Desse modo, claramente todo ideal primo também é semiprimo. Além disso, qualquer interseção de ideais semiprimos é um ideal semiprimo. De fato, se $\left\{\mathcal{Q}_{\beta} \mid \beta \in \Psi\right\}$ é uma família de ideais semiprimos de $A$ e $I$ um ideal de $A$ tal que $I^{2} \subseteq \bigcap_{\beta} \mathcal{Q}_{\beta}$, então, para todo $\beta \in \Psi$, temos $I^{2} \subseteq \mathcal{Q}_{\beta}$ e portanto, $I \subseteq \mathcal{Q}_{\beta}$, isto é, $I \subseteq \bigcap_{\beta} \mathcal{Q}_{\beta}$. Observe também que a definição de ideal semiprimo dada acima é equivalente a "se $I$ é um ideal de $A$ tal que $I^{n} \subseteq \mathcal{Q}$, para algum $n \in \mathbb{N}$, então, $I \subseteq \mathcal{Q}$ ".

Proposição 1.1.4. Um ideal $\mathcal{Q}$ de um anel $A$ é semiprimo se, e somente se, o anel quociente $A / \mathcal{Q}$ não contém ideais nilpotentes não nulos, isto é, $A / \mathcal{Q}$ é um anel semiprimo.

Demonstração. Assuma que $\mathcal{Q}$ é um ideal semiprimo de $A$ e seja $U$ um ideal nilpotente de $A / \mathcal{Q}$. Assim, existe $n \in \mathbb{N}$ tal que $U^{n}=0$. Considere a projeção natural $\pi: A \rightarrow A / \mathcal{Q}$. Desse modo, $\pi^{-1}\left(U^{n}\right)=\pi^{-1}(0)=\mathcal{Q}$ e segue $\pi^{-1}(U)^{n} \subseteq \pi^{-1}\left(U^{n}\right)=\mathcal{Q}$, pois se $x \in \pi^{-1}(U)^{n}$, então, $x=$ $\sum_{i} x_{1}^{i} \ldots x_{n}^{i}$, onde $x_{j}^{i} \in \pi^{-1}(U)$, o que implica $\pi(x)=\sum_{i} \pi\left(x_{1}^{i}\right) \ldots \pi\left(x_{n}^{i}\right) \in U^{n}$. Como $\mathcal{Q}$ é semiprimo, temos $\pi^{-1}(U) \in \mathcal{Q}$ e portanto, podemos concluir $U=\pi\left(\pi^{-1}(U)\right) \subseteq \pi(\mathcal{Q})=0$, pois $\pi$ é sobrejetora. Reciprocamente, suponha que $A / \mathcal{Q}$ é um anel semiprimo e que $I$ é um ideal de $A$ tal que $I^{2} \subseteq \mathcal{Q}$. Neste caso, $\pi(I)^{2} \subseteq \pi\left(I^{2}\right) \subseteq \pi(\mathcal{Q})=0$, e como $\pi$ é sobrejetora, segue que $\pi(I)$ é um ideal de $A / \mathcal{Q}$. Portanto, por hipótese, concluímos $\pi(I)=0$ e $I \subseteq \pi^{-1}(0)=\mathcal{Q}$.

Teorema 1.1.3. Se $\mathcal{Q}$ é um ideal de um anel $A$, então, as seguintes condições são equivalentes:

(i) $\mathcal{Q}$ é um ideal semiprimo;

(ii) se $a \in A$ é tal que $a A a \subseteq \mathcal{Q}$, então, $a \in \mathcal{Q}$; 
(iii) se (a) é um ideal principal de $A$ tal que $(a)^{2} \subseteq \mathcal{Q}$, então, $a \in \mathcal{Q}$;

(iv) se $U$ é um ideal à direita de $A$ tal que $U^{2} \subseteq \mathcal{Q}$, então, $U \subseteq \mathcal{Q}$;

(v) se $U$ é um ideal à esquerda de $A$ tal que $U^{2} \subseteq \mathcal{Q}$, então, $U \subseteq \mathcal{Q}$.

Demonstração. (i) $\Rightarrow$ (ii) Se $a \in A$ é tal que $a A a \subseteq \mathcal{Q}$, então, $A a A a A \subseteq \mathcal{Q}$ e $(A a A)(A a A) \subseteq \mathcal{Q}$. Assim, como $A a A$ é um ideal de $A$, temos $A a A \subseteq \mathcal{Q}$. Agora, se $I=(a)$, concluímos que valem $I^{3} \subseteq A a A \subseteq \mathcal{Q}$, o que implica $I \subseteq \mathcal{Q}$. Portanto, $a \in \mathcal{Q}$.

(ii) $\Rightarrow$ (iii) Suponha que (a) seja um ideal principal tal que $(a)^{2} \subseteq \mathcal{Q}$. Desse modo, $a A a \subseteq(a)^{2} \subseteq \mathcal{Q}$ e portanto, $a \in \mathcal{Q}$.

(iii) $\Rightarrow$ (iv) Se $U$ é um ideal à direita satisfazendo $U^{2} \subseteq \mathcal{Q}$ e $u \in U$, temos $(u)^{2} \subseteq U^{2}+A U^{2} \subseteq \mathcal{Q}$ o que, por hipótese, implica $u \in \mathcal{Q}$, de onde segue $U \subseteq \mathcal{Q}$.

(iii) $\Rightarrow$ (v) Se $U$ é um ideal à esquerda de $A$ tal que $U^{2} \subseteq \mathcal{Q}$ e $u \in U$, então, $(u)^{2} \subseteq U^{2}+U^{2} A \subseteq \mathcal{Q}$, o que implica $u \in \mathcal{Q}$, por hipótese. Portanto, $U \subseteq \mathcal{Q}$.

Finalmente, assumindo (iv) ou (v), temos que se $I$ é um ideal de $A$ tal que $I^{2} \subseteq \mathcal{Q}$, então, em particular, $I$ é um ideal à direita e à esquerda. Portanto, $I \subseteq \mathcal{Q}$, e podemos concluir que $\mathcal{Q}$ é ideal semiprimo.

Proposição 1.1.5. Um anel $A$ é semiprimo se, e somente se, satisfaz a seguinte propriedade:

"se $a \in A$ é um elemento tal que $a A a=0$, então, $a=0 "$ "

Demonstração. Um anel $A$ é semiprimo se, e somente se, $A / 0 \cong A$ é um anel semiprimo, se, e somente se, 0 é um ideal semiprimo, o que equivale à propriedade de (1.3), pelo Teorema 1.1.3.

Definição 1.1.7. Um subconjunto $N$ de um anel $A$ é um $\mathbf{n}$-sistema se satisfaz a seguinte propriedade:

"se $a \in N$, então, existe $x \in A$ tal que $a x a \in N "$.

Claramente todo m-sistema de $A$ também é um n-sistema.

Lema 1.1.3. Um ideal $\mathcal{Q}$ é um ideal semiprimo de um anel $A$ se, e somente se, $C(\mathcal{Q})$, o complementar de $\mathcal{Q}$ em $A$, é um n-sistema.

Demonstração. Suponha que $\mathcal{Q}$ é um ideal semiprimo. Pelo Teorema 2.2.1, temos que se $a \in A$ é um elemento tal que $a A a \subseteq \mathcal{Q}$, então, $a \in \mathcal{Q}$. Assim, se $a \in C(\mathcal{Q})$, isto é, $a \notin \mathcal{Q}$, temos $a A a \nsubseteq \mathcal{Q}$. Desse modo, existe $x \in A$ tal que axa $\notin \mathcal{Q}$, ou seja, axa $\in C(\mathcal{Q})$. Reciprocamente, assuma que $C(\mathcal{Q})$ é um n-sistema e seja $a \in A$ tal que $a A a \subseteq \mathcal{Q}$. Se $a \notin \mathcal{Q}$, então, existe $x \in A$ tal que $a x a \notin \mathcal{Q}$, o que é uma contradição. Logo, $a \in \mathcal{Q}$ e $\mathcal{Q}$ é um ideal semiprimo.

Lema 1.1.4. Se $N$ é um n-sistema de um anel $A$ e $a \in N$, então, existe um m-sistema $M$ de $A$ tal que $a \in M$ e $M \subseteq N$.

Demonstração. Vamos construir um $m$-sistema $M=\left\{a_{1}, a_{2}, a_{3}, \ldots\right\}$. Inicialmente, seja $a_{1}=a$. Como $a_{1} \in N$, então, $a_{1} A a_{1} \cap N \neq \emptyset$. Assim, seja $a_{2}$ um elemento qualquer de $a_{1} A a_{1} \cap N$. De modo geral, se $a_{i}$ foi definido, como $a_{i} \in N$, podemos escolher $a_{i+1}$ como um elemento de $a_{i} A a_{i} \cap N \neq \emptyset$. 
Portanto, $a \in M$ e $M \subseteq N$. Desse modo, somente falta mostrar que $M$ é um m-sistema. Para isso, sejam $a_{i}, a_{j} \in M$. Assumindo, sem perda de generalidade, $i \leq j$, temos $a_{j+1} \in a_{j} A a_{j} \subseteq a_{i} A a_{j}$, isto é, $a_{j+1} \in a_{i} A a_{j} \cap M \neq \emptyset$.

Vamos usar agora os resultados provados para o radical primo de um ideal de um anel $A$.

Teorema 1.1.4. Um ideal $\mathcal{Q}$ de um anel $A$ é semiprimo se, e somente se, $\mathcal{B}(\mathcal{Q})=\mathcal{Q}$.

Demonstração. Se $\mathcal{B}(\mathcal{Q})=\mathcal{Q}$, então, $\mathcal{Q}$ é uma interseção de ideais primos e consequentemente, é uma interseção de ideais semiprimos, o que implica que $\mathcal{Q}$ é semiprimo. Reciprocamente, assuma que $\mathcal{Q}$ é semiprimo. Claramente, $\mathcal{Q} \subseteq \mathcal{B}(\mathcal{Q})$. Suponha que esta inclusão seja estrita e tome $a \in \mathcal{B}(\mathcal{Q})$ tal que $a \notin \mathcal{Q}$. Assim, $C(\mathcal{Q})$ é um n-sistema e $a \in C(\mathcal{Q})$, logo existe $M$ um m-sistema tal que $a \in M$ e $M \subseteq C(\mathcal{Q})$. Como $a \in \mathcal{B}(\mathcal{Q})$, temos também $M \cap \mathcal{Q} \neq \emptyset$, o que é uma contradição com a inclusão $M \subseteq C(\mathcal{Q})$. Portanto, $\mathcal{Q}=\mathcal{B}(\mathcal{Q})$.

Corolário 1.1.1. Um ideal $\mathcal{Q}$ de um anel $A$ é semiprimo se, e somente se, $\mathcal{Q}$ é uma interseção de ideais primos de $A$.

Agora, facilmente concluímos o resultado que queríamos. Se $A$ é um anel semiprimo, então, $A / 0 \cong A$ é semiprimo, e portanto, 0 é um ideal semiprimo de $A$. Desse modo, 0 é uma interseção de ideais primos de $A(0=\mathcal{B}(0))$.

Definição 1.1.8. O centro de um anel $A$ é o conjunto

$$
Z(A)=\{x \in A \mid x a=a x, \text { para todo } a \in A\}
$$

Os seguintes lemas desempenham um papel importante no quarto capítulo.

Lema 1.1.5. Se $A$ é um anel semiprimo e $a \in A$ é um elemento nilpotente pertencente a $Z(A)$, então, $a=0$. Em outras palavras, o centro de $A$ não contém elementos nilpotentes não nulos.

Demonstração. Seja $n$ o índice de nilpotência de $a$, isto é, $a^{n}=0$ e $a^{n-1} \neq 0$. Se $n>1$, então, para qualquer $x \in A$, temos $a^{n-1} x a^{n-1}=a^{n} x a^{n-2}=0$, pois $a \in Z(A)$, o que implica $a^{n-1}=0$, pois $A$ é semiprimo; o que é uma contradição. Portanto, $n=1$ e $a=0$.

Lema 1.1.6. Sejam $A$ um anel semiprimo de característica diferente de 2 e $a, b \in A$. Se

$$
a x b+b x a=0, \text { para todo } x \in A,
$$

então, $a x b=b x a=0$, para todo $x \in A$.

Demonstração. Sejam $x$ e $y$ elementos quaisquer de $A$. Pela equação (1.4), temos $a x b=-b x a$ e $a y b=-b y a$. Usando essas relações repetidas vezes, obtemos

$$
(a x b) y(a x b)=-[b(x a y) a] x b=a x(a y b) x b=-(a x b) y(a x b) .
$$

Assim, $2(a x b) y(a x b)=0$, o que resulta em $(a x b) y(a x b)=0$, pois a característica de $A$ é diferente de 2. Logo, como $y$ é qualquer e $A$ é semiprimo, podemos concluir que valem $a x b=0$, e $b x a=-a x b=0$, para todo $x \in A$, como queríamos. 
Lema 1.1.7. Se $A$ é um anel semiprimo de característica diferente de 2 e $a \in A$ um elemento tal que $[a,[a, x]]=0$, para todo $x \in A$, então, $a \in Z(A)$.

Demonstração. Seja $d: A \rightarrow A$ uma função tal que $d(x)=x a-a x$, para todo $x \in A$. Assim,

$$
\begin{aligned}
& d(x+y)=(x+y) a-a(x+y)=(x a-a x)+(y a-a y)=d(x)+d(y) e \\
& d(x y)=(x y) a-a(x y)=(x a) y-(a x) y+x(y a)-x(a y)=d(x) y+x d(y),
\end{aligned}
$$

para todos $x, y \in A$, o que quer dizer que $d$ é uma derivação, como poderá ser visto no próximo capítulo. Além disso,

$$
d^{2}(x)=d(d(x))=d(x a-a x)=(x a-a x) a-a(x a-a x)=[a,[a, x]]=0,
$$

para todo $x \in A$, o que implica

$$
\begin{aligned}
0=d^{2}(x y) & =d(d(x y))=d(d(x) y+x d(y)) \\
& =d(d(x) y)+d(x d(y)) \\
& =d^{2}(x) y+d(x) d(y)+d(x) d(y)+x d^{2}(y) \\
& =2 d(x) d(y) .
\end{aligned}
$$

Portanto, $d(x) d(y)=0$, para todos $x, y \in A$, pois a característica de $A$ é diferente de 2 . Agora, escolhendo $y=r x$, onde $r \in A$, temos $d(y)=d(r) x+r d(x)$, e assim, $0=d(x) d(r x)=$ $d(x)[d(r) x+r d(x)]=d(x) r d(x)$, pois $d(x) d(r)=0$. Desse modo, $d(x) A d(x)=0$, e como $A$ é semiprimo, podemos concluir $d(x)=0$, para todo $x \in A$, ou seja, $x a-a x=0$, para todo $x \in A$. Logo, $a \in Z(A)$.

\subsection{Superálgebras primas, semiprimas e ideais graduados}

Ao longo de toda a dissertação, entendemos álgebra como álgebra sobre um anel comutativo $\Phi$ com identidade e tal que $\frac{1}{2} \in \Phi$, caso contrário diremos explicitamente.

Definição 1.2.1. Uma superálgebra $\mathcal{A}$ é uma álgebra $\mathbb{Z}_{2}$-graduada, isto é, existem submódulos $\mathcal{A}_{0}$ e $\mathcal{A}_{1}$ de $\mathcal{A}$ tais que $\mathcal{A}=\mathcal{A}_{0} \oplus \mathcal{A}_{1}$ e $\mathcal{A}_{0} \mathcal{A}_{0} \subseteq \mathcal{A}_{0}$ (e portanto, $\mathcal{A}_{0}$ é uma subálgebra de $\mathcal{A}$ ), $\mathcal{A}_{0} \mathcal{A}_{1} \subseteq \mathcal{A}_{1}$, $\mathcal{A}_{1} \mathcal{A}_{0} \subseteq \mathcal{A}_{1}$ e $\mathcal{A}_{1} \mathcal{A}_{1} \subseteq \mathcal{A}_{0}$.

Dizemos que $\mathcal{A}_{0}$ é a parte par e $\mathcal{A}_{1}$ é a parte ímpar de $\mathcal{A}$. Quando $\mathcal{A}_{1}=0$, a superálgebra $\mathcal{A}$ é chamada de trivial. Se $a \in \mathcal{A}_{i}, i=0$ ou $i=1$, então, $a$ é dito um elemento homogêneo de grau $i \mathrm{e}$ denotamos $|a|=i$. Uma superálgebra associativa é uma álgebra associativa $\mathbb{Z}_{2}$-graduada.

Um submódulo (ideal) $\mathcal{B}$ de $\mathcal{A}$ é dito um submódulo (ideal) graduado se $\mathcal{B}=\mathcal{B}_{0} \oplus \mathcal{B}_{1}$, onde $\mathcal{B}_{i}=\mathcal{B} \cap \mathcal{A}_{i}, i=0,1$.

Dada uma superálgebra $\mathcal{A}=\mathcal{A}_{0} \oplus \mathcal{A}_{1}$, temos que a aplicação $\sigma: \mathcal{A} \rightarrow \mathcal{A}$ tal que $\sigma\left(a_{0}+a_{1}\right)=$ $a_{0}-a_{1}$ é um automorfismo de $\mathcal{A}$ satisfazendo $\sigma^{2}=$ id. Reciprocamente, dada uma álgebra $\mathcal{A}$ e um automorfismo $\sigma$ de $\mathcal{A}$ tal que $\sigma^{2}=$ id, podemos munir $\mathcal{A}$ de uma $\mathbb{Z}_{2}$-graduação da seguinte maneira: definimos $\mathcal{A}_{0} \doteq\{a \in \mathcal{A} \mid \sigma(a)=a\}$ e $\mathcal{A}_{1} \doteq\{a \in \mathcal{A} \mid \sigma(a)=-a\}$, então, $\mathcal{A}_{0}$ e $\mathcal{A}_{1}$ são submódulos de $\mathcal{A}$ satisfazendo as condições da Definição 1.2.1. Com efeito, para qualquer elemento $a$ de $\mathcal{A}$, podemos 
escrever $a=\frac{a+\sigma(a)}{2}+\frac{a-\sigma(a)}{2}$. Desse modo, é possível caracterizar a $\mathbb{Z}_{2}$-graduação pelo automorfismo $\sigma$.

Exemplo 1.2.1. Sejam $\mathcal{A}$ uma álgebra e $c \in \mathcal{A}$ um elemento invertível tal que $c^{2} \in Z(\mathcal{A})$. Defina $\sigma: \mathcal{A} \rightarrow \mathcal{A}$ por $\sigma(a)=c a c^{-1}$, para todo $a \in \mathcal{A}$. Assim, $\sigma$ é um automorfismo de $\mathcal{A}$ tal que $\sigma^{2}(a)=\sigma\left(c a c^{-1}\right)=c^{2} a c^{-2}=a c^{2} c^{-2}=\operatorname{id}(a)$, para todo $a \in \mathcal{A}$, o que nos permite concluir que $\mathcal{A}=\mathcal{A}_{0} \oplus \mathcal{A}_{1}$ é superálgebra, onde $\mathcal{A}_{0}=\{a \in \mathcal{A} \mid c a=a c\}$ e $\mathcal{A}_{1}=\{a \in \mathcal{A} \mid c a=-a c\}$.

Exemplo 1.2.2. Seja $\mathcal{A}=A \times A$, onde $A$ é uma álgebra. Considerando $\sigma: \mathcal{A} \rightarrow \mathcal{A}$ tal que $\sigma(a, b)=(b, a)$, para todo $(a, b) \in \mathcal{A}$, temos que $\sigma$ é um automorfismo de $\mathcal{A}$, tal que $\sigma^{2}=$ id. Portanto, $\mathcal{A}=\mathcal{A}_{0} \oplus \mathcal{A}_{1}$ é uma superálgebra, onde $\mathcal{A}_{0}=\{(a, a) \mid a \in A\}$ e $\mathcal{A}_{1}=\{(a,-a) \mid a \in A\}$. Neste caso, dizemos que a superálgebra é dada pelo automorfismo de troca.

Lema 1.2.1. Um submódulo $\mathcal{B}$ de uma superálgebra $\mathcal{A}$ é graduado se, e somente se, $\sigma(\mathcal{B})=\mathcal{B}$.

Demonstração. $(\Rightarrow)$ Seja $\mathcal{B}$ seja um submódulo graduado de $\mathcal{A}$, isto é, $\mathcal{B}=\mathcal{B}_{0} \oplus \mathcal{B}_{1}$, onde $\mathcal{B}_{i}=$ $\mathcal{B} \cap \mathcal{A}_{i}, i=0,1$. Dado $b \in \mathcal{B}$, temos $\sigma(b)=\sigma\left(b_{0}+b_{1}\right)=b_{0}-b_{1} \in \mathcal{B}$, pois $b_{0} \in \mathcal{B}_{0}$ e $b_{1} \in \mathcal{B}_{1}$ e $\mathcal{B}$ é um submódulo. Além disso, $b=\mathrm{id}(b)=\sigma^{2}(b)=\sigma(\sigma(b)) \in \sigma(\mathcal{B})$, isto é, $\mathcal{B} \subseteq \sigma(\mathcal{B})$. Finalmente, se $b \in \sigma(\mathcal{B})$, então, $b=\sigma(\bar{b})$, com $\bar{b} \in \mathcal{B}$, e portanto, pelos mesmos argumentos, $b \in \mathcal{B}$. Portanto, $\sigma(\mathcal{B})=\mathcal{B}$.

$(\Leftarrow)$ Suponha $\sigma(\mathcal{B})=\mathcal{B}$. Dado $b \in \mathcal{B} \subseteq \mathcal{A}$, podemos escrever $b=b_{0}+b_{1}$, onde $b_{i} \in \mathcal{A}_{i}, i=0,1$. Assim, $\sigma(b)=b_{0}-b_{1} \in \mathcal{B}$. Como $\mathcal{B}$ é submódulo, temos $b+\sigma(b)=2 b_{0} \in \mathcal{B}$, o que implica $b_{0} \in \mathcal{B}$ e $b-\sigma(b)=2 b_{1} \in \mathcal{B}$. Portanto, $b_{1} \in \mathcal{B}$. Desse modo, $b_{i} \in \mathcal{B} \cap \mathcal{A}_{i}, i=0,1$, isto é, $\mathcal{B}=\mathcal{B}_{0} \oplus \mathcal{B}_{1}$.

Exemplo 1.2.3. O centro de uma superálgebra associativa $\mathcal{A}, Z(\mathcal{A})$, é um submódulo graduado de $\mathcal{A}$. De fato, dado $\sigma(a) \in \sigma(Z(\mathcal{A})$ ), para cada $b \in \mathcal{A}$, sendo $\sigma$ um automorfismo, existe um $\bar{b} \in \mathcal{A}$ tal que $\sigma(\bar{b})=b$. Assim, $\sigma(a) b=\sigma(a) \sigma(\bar{b})=\sigma(a \bar{b})=\sigma(\bar{b} a)=\sigma(\bar{b}) \sigma(a)=b \sigma(a)$, isto é, $\sigma(Z(\mathcal{A})) \subseteq Z(\mathcal{A})$. Por outro lado, se $a \in Z(\mathcal{A})$, então, existe um $\bar{a} \in \mathcal{A}$ tal que $\sigma(\bar{a})=a$, mas

$$
\sigma(\bar{a} b)=\sigma(\bar{a}) \sigma(b)=a \sigma(b)=\sigma(b) a=\sigma(b) \sigma(\bar{a})=\sigma(b \bar{a})
$$

implica $\bar{a} b=b \bar{a}$, para todo $b \in \mathcal{A}$, pois $\sigma$ é injetora. Desse modo, $\bar{a} \in Z(\mathcal{A})$ e consequentemente, $a \in \sigma(Z(\mathcal{A}))$ e $Z(\mathcal{A}) \subseteq \sigma(Z(\mathcal{A}))$.

Definição 1.2.2. Uma superálgebra $\mathcal{A}$ é uma superálgebra prima se o produto de quaisquer dois ideais graduados não nulos de $\mathcal{A}$ é um ideal não nulo.

É importante observar que uma superálgebra prima não é necessariamente uma álgebra prima.

Exemplo 1.2.4. Se na superálgebra $\mathcal{A}$ dada no Exemplo 1.2.2, $A$ é uma álgebra prima, então, $\mathcal{A}$ é uma superálgebra prima, mas não é uma álgebra prima. De fato, $(0 \times A)(A \times 0)=0,0 \times A$ e $A \times 0$ são não nulos, mas não são graduados, pois $\sigma(0 \times A)=A \times 0$ e $\sigma(A \times 0)=0 \times A$.

Lema 1.2.2. Uma superálgebra associativa $\mathcal{A}$ é uma superálgebra prima se, e somente se, satisfaz a seguinte propriedade:

"se $a, b \in \mathcal{A}$ são elementos homogêneos de $\mathcal{A}$ tais que $a \mathcal{A} b=0$, então, $a=0$ ou $b=0$ ". 
Demonstração. Sejam $\mathcal{A}$ uma superálgebra associativa prima e $a, b \in \mathcal{A}_{0} \cup \mathcal{A}_{1}$ tais que $a \mathcal{A} b=0$. Vamos supor, sem perda de generalidade, $a=a_{0} \in \mathcal{A}_{0}$ e $b=b_{1} \in \mathcal{A}_{1}$, pois os outros casos seguem de maneira análoga. Observe que vale $\left(\mathcal{A} a_{0} \mathcal{A}\right)\left(\mathcal{A} b_{1} \mathcal{A}\right) \subseteq \mathcal{A}\left(a_{0} \mathcal{A} b_{1}\right) \mathcal{A}=0$, isto é, $\mathcal{A} a_{0} \mathcal{A}$ e $\mathcal{A} b_{1} \mathcal{A}$ são ideais de $\mathcal{A}$ tais que seu produto é nulo. Além disso, estes ideais são graduados. Com efeito, se $x a_{0} y \in \mathcal{A} a_{0} \mathcal{A}$, então,

$$
\begin{aligned}
\sigma\left(x a_{0} y\right) & =\sigma\left(x_{0} a_{0} y_{0}+x_{1} a_{0} y_{1}+x_{0} a_{0} y_{1}+x_{1} a_{0} y_{0}\right) \\
& =x_{0} a_{0} y_{0}+x_{1} a_{0} y_{1}-x_{0} a_{0} y_{1}-x_{1} a_{0} y_{0} \\
& =\sigma(x) a_{0} y_{0}+\sigma(x) a_{0}\left(-y_{1}\right) \\
& =\sigma(x) a_{0} \sigma(y) \in \mathcal{A} a_{0} \mathcal{A},
\end{aligned}
$$

onde $\sigma$ é o automorfismo definido pela $\mathbb{Z}_{2}$-graduação de $\mathcal{A}$; o que prova $\sigma\left(\mathcal{A} a_{0} \mathcal{A}\right) \subseteq \mathcal{A} a_{0} \mathcal{A}$. Assim, aplicando $\sigma$ nesta última inclusão, segue $\mathcal{A} a_{0} \mathcal{A} \subseteq \sigma\left(\mathcal{A} a_{0} \mathcal{A}\right)$, pois $\sigma^{2}=$ id. Do mesmo modo, podemos provar que $\mathcal{A} b_{1} \mathcal{A}$ é graduado. Como $\mathcal{A}$ é uma superálgebra prima, concluímos que vale $\mathcal{A} a_{0} \mathcal{A}=0$ ou $\mathcal{A} b_{1} \mathcal{A}=0$. Agora, sejam $I=$ ideal $\left\langle a_{0}\right\rangle$ e $J=$ ideal $\left\langle b_{1}\right\rangle$ os ideais de $\mathcal{A}$ gerados por $a_{0}$ e $b_{1}$, respectivamente. Desse modo, $I^{3} \subseteq \mathcal{A} a_{0} \mathcal{A}$ e $J^{3} \subseteq \mathcal{A} b_{1} \mathcal{A}$, e portanto, $I^{3}=0$ ou $J^{3}=0$, o que novamente pela hipótese de $\mathcal{A}$ ser prima implica $I=0$ ou $J=0$. Logo, $a_{0}=0$ ou $b_{1}=0$.

Reciprocamente, suponha que $\mathcal{A}$ satisfaz a propriedade inicial. Sejam $I$ e $J$ ideais graduados de $\mathcal{A}$ tais que $I J=0$ e suponha $I \neq 0$. Desse modo, existe $0 \neq x=x_{0}+x_{1} \in I$. Podemos admitir, sem perda de generalidade, $x_{0} \neq 0$. Como $I$ é graduado, temos $\sigma(I)=I$ e portanto,

$$
0 \neq x_{0}=\frac{1}{2}(x+\sigma(x)) \in I .
$$

Assim, para todo $y \in J$, temos $x_{0} y=0$, o que implica $x_{0} \mathcal{A} y=0$, pois $J$ é ideal. Em particular, para $y_{0} \in J_{0}=J \cap \mathcal{A}_{0}$, segue $x_{0} \mathcal{A} y_{0}=0$ e pela propriedade inicial, concluímos $y_{0}=0$. Analogamente, para $y_{1} \in J_{1}=J \cap \mathcal{A}_{1}$, temos $x_{0} \mathcal{A} y_{1}=0$, o que implica $y_{1}=0$. Logo, $J=J_{0} \oplus J_{1}=0$, pois $J$ é graduado. Portanto, $\mathcal{A}$ é uma superálgebra prima.

Definição 1.2.3. Uma superálgebra $\mathcal{A}$ é uma superálgebra semiprima se não possui ideais graduados nilpotentes não nulos.

Em analogia ao Lema 1.2.2, temos o seguinte

Lema 1.2.3. Uma superálgebra associativa $\mathcal{A}$ é uma superálgebra semiprima se, e somente se, satisfaz a seguinte propriedade:

"se $a \in \mathcal{A}$ é um elemento homogêneo tal que $a \mathcal{A} a=0$, então, $a=0$ ".

Demonstração. Sejam $\mathcal{A}$ uma superálgebra associativa semiprima e $a \in \mathcal{A}_{0} \cup \mathcal{A}_{1}$ tal que $a \mathcal{A} a=$ 0 . Vamos supor, sem perda de generalidade, $a=a_{0} \in \mathcal{A}_{0}$. Note que vale $\left(\mathcal{A} a_{0} \mathcal{A}\right)\left(\mathcal{A} a_{0} \mathcal{A}\right) \subseteq$ $\mathcal{A}\left(a_{0} \mathcal{A} a_{0}\right) \mathcal{A}=0$, isto é, $\mathcal{A} a_{0} \mathcal{A}$ é um ideal nilpotente de $\mathcal{A}$. De fato, este ideal é graduado e como $\mathcal{A}$ é uma superálgebra semiprima, segue $\mathcal{A} a_{0} \mathcal{A}=0$. Definindo agora $I=$ ideal $\left\langle a_{0}\right\rangle$, o ideal de $\mathcal{A}$ gerado por $a_{0}$, temos $I^{3} \subseteq \mathcal{A} a_{0} \mathcal{A}=0$, o que implica $I=0$, ou seja, $a_{0}=0$.

Reciprocamente, assuma que $\mathcal{A}$ satisfaz a propriedade inicial. É suficiente mostrar que $\mathcal{A}$ não possui ideais graduados não nulos cujo quadrado é nulo. Desse modo, considere $I$ um ideal graduado de $\mathcal{A}$ tal que $I^{2}=0$. Para $x_{0} \in I_{0}=I \cap \mathcal{A}_{0}$, temos $x_{0} \mathcal{A} x_{0} \subseteq I^{2}=0$ e portanto, $x_{0}=0$. 
Analogamente, se $x_{1} \in I_{1}=I \cap \mathcal{A}_{1}$, então, $x_{1} \mathcal{A} x_{1} \subseteq I^{2}=0$, o que implica $x_{1}=0$. Como $I$ é graduado, segue $I=I_{0} \oplus I_{1}=0$. Logo, $\mathcal{A}$ é uma superálgebra semiprima.

Portanto, toda superálgebra prima é uma superálgebra semiprima. O próximo lema "conecta" as estruturas de superálgebra prima e de álgebra prima.

Lema 1.2.4. Seja $\mathcal{A}=\mathcal{A}_{0} \oplus \mathcal{A}_{1}$ uma superálgebra associativa. Se $\mathcal{A}$ é superálgebra semiprima, então, $\mathcal{A}$ e $\mathcal{A}_{0}$ são álgebras semiprimas. Se $\mathcal{A}$ é superálgebra prima, então, $\mathcal{A}$ é álgebra prima ou $\mathcal{A}_{0}$ é álgebra prima.

Demonstração. Suponha que $\mathcal{A}$ é superálgebra semiprima. Seja $\sigma$ o automorfismo de $\mathcal{A}$ definido pela $\mathbb{Z}_{2}$-graduação da superálgebra, isto é, $\sigma\left(a_{0}+a_{1}\right)=a_{0}-a_{1}$, para todo $a_{0}+a_{1} \in \mathcal{A}$, e seja $I$ um ideal de $\mathcal{A}$ tal que $I^{2}=0$. Afirmamos que $I \cap \sigma(I)$ é um ideal graduado de $\mathcal{A}$ tal que $(I \cap \sigma(I))^{2}=0$. De fato,

- $I \cap \sigma(I)$ é ideal, pois como $\sigma$ é um automorfismo temos que $\sigma(I)$ é ideal;

- se $a \in I \cap \sigma(I)$, então, $a \in I$ e $a=\sigma(b)$, para algum $b \in I$, o que implica $\sigma(a)=\sigma^{2}(b)=b \in$ $\sigma(I)$, ou seja, $a \in \sigma(I \cap \sigma(I))$; além disso, se $a \in \sigma(I \cap \sigma(I))$, então, $a=\sigma(b)$, para algum $b \in I \cap \sigma(I)$, o que implica $a \in \sigma(I)$ e $a=\sigma(b)=\sigma(\sigma(c))=c \in I$, isto é, $a \in I$;

- como $I \cap \sigma(I) \subseteq I$ e $I^{2}=0$, temos $(I \cap \sigma(I))^{2}=0$.

Além disso, como $\mathcal{A}$ é uma superálgebra semiprima e $I \cap \sigma(I)$ é um ideal graduado nilpotente, temos $I \cap \sigma(I)=0$. Consequentemente,

$$
\begin{aligned}
& I \sigma(I) \subseteq I \cap \sigma(I)=0 \text { implica } I \sigma(I)=0, \\
& \sigma(I) I \subseteq I \cap \sigma(I)=0 \text { implica } \sigma(I) I=0 .
\end{aligned}
$$

Sejam $a, b \in I+\sigma(I), a=a_{1}+a_{2}$ e $b=b_{1}+b_{2}$, onde $a_{1}, b_{1} \in I$ e $a_{2}, b_{2} \in \sigma(I)$. Desse modo, temos $a_{1} b_{2}=0$ e $a_{2} b_{1}=0$. Assim, $a b=a_{1} b_{1}+a_{1} b_{2}+a_{2} b_{1}+a_{2} b_{2}=a_{1} b_{1}+a_{2} b_{2}=0$, pois $a_{1} b_{1} \in I^{2}=0$ e $a_{2} b_{2} \in \sigma(I)^{2}=\sigma\left(I^{2}\right)=0$. Consequentemente, $(I+\sigma(I))^{2}=0$; e como $\sigma^{2}=$ id, este último é um ideal graduado de $\mathcal{A}$, e segue $I+\sigma(I)=0$. Portanto, podemos concluir $I=0$, pois $I \subseteq I+\sigma(I)=0$. Logo, $\mathcal{A}$ é uma álgebra semiprima.

Prosseguindo, seja $I$ um ideal de $\mathcal{A}_{0}$ tal que $I^{2}=0$. Se $x_{1}, y_{1} \in \mathcal{A}_{1}$ e $a, b, c \in I \subseteq \mathcal{A}_{0}$, temos $a x_{1} b y_{1} c=a\left(x_{1} b y_{1}\right) c \in I \mathcal{A}_{0} I$, o que implica $I \mathcal{A}_{1} I \mathcal{A}_{1} I \subseteq I \mathcal{A}_{0} I \subseteq I^{2}=0$, isto é, $I \mathcal{A}_{1} I \mathcal{A}_{1} I=0$. Agora, considere $J$ o ideal de $\mathcal{A}$ gerado por $I$, o qual podemos descrever como

$$
J=\mathcal{A} I+I \mathcal{A}+\mathcal{A} I \mathcal{A}+\Phi I+\mathbb{Z} I
$$

e então, das igualdades $I^{2}=0$ e $I \mathcal{A}_{1} I \mathcal{A}_{1} I=0$, concluímos $J^{3}=0$, e consequentemente, $J=0$, o que implica $I=0$. Portanto, $\mathcal{A}_{0}$ é uma álgebra semiprima.

Suponha agora que $\mathcal{A}$ é superálgebra prima e que $\mathcal{A}$ não é álgebra prima, então, podemos tomar um ideal maximal $P$ com respeito à propriedade $P \cap \sigma(P)=0$. De fato, basta definir o conjunto $\Omega=\{I$ ideal de $\mathcal{A} \mid I \cap \sigma(I)=0\}$ ordenado parcialmente pela inclusão de conjuntos e aplicar o Lema de Zorn. Note que $P$ é um ideal não nulo. Com efeito, como $\mathcal{A}$ não é uma álgebra prima, 
existem ideais $I$ e $J$ não nulos de $\mathcal{A}$ tais que $I J=0$, o que implica que os ideais graduados $I \cap \sigma(I)$ e $J \cap \sigma(J)$ satisfazem $(I \cap \sigma(I))(J \cap \sigma(J))=0$, e, assim, $I \cap \sigma(I)=0$ ou $J \cap \sigma(J)=0$, isto é, $0 \neq I \in \Omega$ ou $0 \neq J \in \Omega$. Além disso, o ideal $P$ é primo. De fato, se $I$ e $J$ são ideais de $\mathcal{A}$ tais que $P \subseteq I, P \subseteq J$ e $I J \subseteq P$, então, $I \cap \sigma(I)$ e $J \cap \sigma(J)$ são ideais graduados de $\mathcal{A}$ tais que $(I \cap \sigma(I))(J \cap \sigma(J)) \subseteq P \cap \sigma(P)=0$, o que implica $I \cap \sigma(I)=0$ ou $J \cap \sigma(J)=0$, ou seja, $I \in \Omega$ ou $J \in \Omega$, pois $\mathcal{A}$ é superálgebra prima. Assim, pela maximalidade de $P$ concluímos $P=I$ ou $P=J$. Portanto, $P$ é um ideal primo. Se $R=P+\sigma(P)$, então, $\sigma(R)=R$ e

$$
\begin{aligned}
& R / P=P+\sigma(P) / P \cong \sigma(P) / P \cap \sigma(P) \cong \sigma(P), \\
& R / \sigma(P)=P+\sigma(P) / \sigma(P) \cong P / P \cap \sigma(P) \cong P,
\end{aligned}
$$

isto é, $R \cong R / P \oplus R / \sigma(P)$. Assim, como $R / \sigma(P) \cong R / P$, podemos concluir $R \cong R / P \oplus R / P$. Munindo $R$ da $\mathbb{Z}_{2}$-graduação definida pelo automorfismo de "troca", como no Exemplo 1.2.2, vemos que $R_{0} \cong R / P$ é primo, pois $R / P \cong R / \sigma(P) \cong P$ é primo. Desse modo, seja $I$ um ideal de $\mathcal{A}_{0}$ tal que $I \cap R_{0}=0$, então, para todo $x \in P$, temos $I(x+\sigma(x))=0$, pois $R \ni x+\sigma(x)=2 x_{0} \in \mathcal{A}_{0}$, ou seja, $x+\sigma(x) \in R_{0}$. Portanto, $I P=I \sigma(P) \subseteq P \cap \sigma(P)=0$, o que implica $I R=0$. Assim, como $I \subseteq \mathcal{A}_{0}$ e $R$ são ideais graduados, $\mathcal{A}$ é superálgebra prima, e $R \neq 0$, pois $0 \neq P \subseteq R$, podemos concluir $I=0$. Provamos que se $I$ é um ideal não nulo de $\mathcal{A}_{0}$, então, como $I \cap R_{0} \neq 0$, segue que $\mathcal{A}_{0}$ é uma álgebra prima, pois $R_{0}$ é uma álgebra prima. De fato, se $I, J$ são ideais de $\mathcal{A}_{0}$ tais que $I J=0$, então, $I J \cap R_{0}=0$, o que implica $\left(I \cap R_{0}\right)\left(J \cap R_{0}\right) \subseteq I J=0$, de onde segue $I \cap R_{0}=0$ ou $J \cap R_{0}=0$, pois $I \cap R_{0}$ e $J \cap R_{0}$ são ideais de $R_{0}$; e consequentemente, $I=0$ ou $J=0$.

Os seguintes lemas serão usados nas demonstrações dos próximos capítulos.

Lema 1.2.5. Seja $\mathcal{A}=\mathcal{A}_{0} \oplus \mathcal{A}_{1}$ uma superálgebra associativa prima. As seguintes condições são válidas:

(1) Se $a_{1} \in \mathcal{A}_{1}$ é tal que $a_{1} \mathcal{A}_{1} a_{1}=0$, então, $a_{1}=0$;

(2) Se $a_{0} \in \mathcal{A}_{0}$ e $a_{1} \in \mathcal{A}_{1}$ são tais que $a_{0} \mathcal{A}_{0} a_{1}=a_{1} \mathcal{A}_{0} a_{0}=0$ ou $a_{0} \mathcal{A}_{1} a_{1}=a_{1} \mathcal{A}_{1} a_{0}=0$, então, $a_{0}=0$ ou $a_{1}=0$.

Demonstração. (1) Dados $b=b_{0}+b_{1}$ e $c=c_{0}+c_{1}$ elementos quaisquer de $\mathcal{A}$, temos

$$
a_{1} b a_{1} c a_{1}=a_{1}\left(b_{0} a_{1} c_{0}\right) a_{1}+a_{1} b_{0}\left(a_{1} c_{1} a_{1}\right)+\left(a_{1} b_{1} a_{1}\right) c a_{1}=0
$$

pois $a_{1} \mathcal{A}_{1} a_{1}=0$. Desse modo, $a_{1} \mathcal{A} a_{1} \mathcal{A} a_{1}=0$, o que implica $a_{1}=0$, pelo fato que $\mathcal{A}$ é uma superálgebra prima.

(2) Se $a_{0} \mathcal{A}_{0} a_{1}=a_{1} \mathcal{A}_{0} a_{0}=0$, então, $\left(a_{0} \mathcal{A}_{1} a_{1}\right) \mathcal{A}_{0}\left(a_{0} \mathcal{A}_{1} a_{1}\right)=0$. Em decorrência do Lema 1.2.4, como $\mathcal{A}_{0}$ é uma álgebra semiprima, temos $a_{0} \mathcal{A}_{1} a_{1}=0$, de onde segue $a_{0} \mathcal{A} a_{1}=0$, o que implica $a_{0}=0$ ou $a_{1}=0$, pois $\mathcal{A}$ é uma superálgebra prima. Por outro lado, supondo $a_{0} \mathcal{A}_{1} a_{1}=a_{1} \mathcal{A}_{1} a_{0}=0$, vale $\left(a_{0} \mathcal{A}_{0} a_{1} \mathcal{A}_{1} a_{0}\right) \mathcal{A}_{0}\left(a_{0} \mathcal{A}_{0} a_{1} \mathcal{A}_{1} a_{0}\right)=0$, o que implica $a_{0} \mathcal{A}_{0} a_{1} \mathcal{A}_{1} a_{0}=$ 0 e consequentemente, $\left(a_{0} \mathcal{A}_{0} a_{1}\right) \mathcal{A}_{1}\left(a_{0} \mathcal{A}_{0} a_{1}\right)=0$. Desse modo, como $a_{0} \mathcal{A}_{0} a_{1} \subseteq \mathcal{A}_{1}$, por (1), segue $a_{0} \mathcal{A}_{0} a_{1}=0$, que juntamente com a hipótese $a_{0} \mathcal{A}_{1} a_{1}=0$ nos permite concluir $a_{0}=0$ ou $a_{1}=0$. 
Lema 1.2.6. Seja $\mathcal{A}$ uma superálgebra associativa prima cuja parte par $\mathcal{A}_{0}$ é comutativa. As seguintes afirmações são válidas:

(1) Se $\left[\mathcal{A}_{0}, \mathcal{A}_{1}\right]=0$, então, $\mathcal{A}$ é comutativa;

(2) Se $a_{0} \in \mathcal{A}_{0}$ e $0 \neq a_{1} \in \mathcal{A}_{1}$ são tais que $\left[a_{0}, a_{1}\right]=0$, então, $a_{0} \in Z(\mathcal{A})$. Em particular, se $\left[\mathcal{A}_{0}, a_{1}\right]=0$, então, $\mathcal{A}$ é comutativa.

(3) Se $0 \neq a_{1} \in \mathcal{A}_{1}$ é tal que $\left[a_{1}, \mathcal{A}_{1}\right]=0$, então, $\mathcal{A}$ é comutativa.

Demonstração. (1) Note que como $\mathcal{A}_{0}$ é comutativa e $\left[\mathcal{A}_{0}, \mathcal{A}_{1}\right]=0$, basta provar que $\mathcal{A}_{1}$ é comutativa. Dados $x_{1}, y_{1} \in \mathcal{A}_{1}$, como $y_{1} x_{1} \in \mathcal{A}_{0}$, por hipótese temos $0=\left[x_{1}, y_{1} x_{1}\right]=$ $x_{1} y_{1} x_{1}-y_{1} x_{1} x_{1}=\left(x_{1} y_{1}-y_{1} x_{1}\right) x_{1}=\left[x_{1}, y_{1}\right] x_{1}$. Assim, para $a_{0} \in \mathcal{A}_{0}$, usando $\left[x_{1}, y_{1}\right] \in \mathcal{A}_{0}$, obtemos que as igualdades $\left[x_{1}, y_{1}\right] a_{0} x_{1}=a_{0}\left[x_{1}, y_{1}\right] x_{1}=0$ são válidas, isto é, $\left[x_{1}, y_{1}\right] \mathcal{A}_{0} x_{1}=0$. Além disso, para $a_{1} \in \mathcal{A}_{1}$, temos $\left[x_{1}, y_{1}\right] a_{1} x_{1}=a_{1}\left[x_{1}, y_{1}\right] x_{1}=0$, pois $\left[x_{1}, y_{1}\right] \in \mathcal{A}_{0}$ e $\left[\mathcal{A}_{0}, \mathcal{A}_{1}\right]=0$. Portanto, $\left[x_{1}, y_{1}\right] \mathcal{A}_{1} x_{1}=0$, e consequentemente $\left[x_{1}, y_{1}\right] \mathcal{A} x_{1}=0$, de onde concluímos $\left[x_{1}, y_{1}\right]=0$, pois $\mathcal{A}$ é uma superálgebra prima.

(2) Dado $x_{1} \in \mathcal{A}_{1}$, temos

$$
\begin{aligned}
a_{1}\left[a_{0}, x_{1}\right] & =a_{1} a_{0} x_{1}-a_{1} x_{1} a_{0} \\
& =\left(a_{0} a_{1} x_{1}-a_{1} x_{1} a_{0}\right)-\left(a_{0} a_{1} x_{1}-a_{1} a_{0} x_{1}\right) \\
& =\left[a_{0}, a_{1} x_{1}\right]-\left[a_{0}, a_{1}\right] x_{1}=0
\end{aligned}
$$

pois $a_{1} x_{1} \in \mathcal{A}_{0}$ e $\left[a_{0}, a_{1}\right]=0$. Para cada $b_{0} \in \mathcal{A}_{0}$, valem as igualdades

$$
\begin{aligned}
a_{1} b_{0}\left[a_{0}, x_{1}\right] & =a_{1}\left(b_{0} a_{0} x_{1}-b_{0} x_{1} a_{0}\right) \\
& =a_{1}\left(a_{0} b_{0} x_{1}-b_{0} x_{1} a_{0}\right) \\
& =a_{1}\left[a_{0}, b_{0} x_{1}\right]=0,
\end{aligned}
$$

isto é, $a_{1} \mathcal{A}_{0}\left[a_{0}, x_{1}\right]=0$, para todo $x_{1} \in \mathcal{A}_{1}$. Além disso, dado $b_{1} \in \mathcal{A}_{1}$, temos

$$
\begin{aligned}
a_{1} b_{1}\left[a_{0}, x_{1}\right] & =a_{1} b_{1} a_{0} x_{1}-a_{1} b_{1} x_{1} a_{0} \\
& =a_{1} b_{1} a_{0} x_{1}-a_{1} a_{0} b_{1} x_{1}+a_{1} a_{0} b_{1} x_{1}-a_{1} b_{1} x_{1} a_{0} \\
& =a_{1}\left[a_{0}, b_{1} x_{1}\right]-a_{1}\left[a_{0}, b_{1}\right] x_{1}=0,
\end{aligned}
$$

isto é, $a_{1} \mathcal{A}_{1}\left[a_{0}, b_{1}\right]=0$, para todo $b_{1} \in \mathcal{A}_{1}$. Portanto, $a_{1} \mathcal{A}\left[a_{0}, b_{1}\right]=0$, o que implica $\left[a_{0}, b_{1}\right]=0$, pois $\mathcal{A}$ é prima e $a_{1} \neq 0$. Assim, $\left[a_{0}, \mathcal{A}_{1}\right]=0$, e como $\mathcal{A}_{0}$ é comutativa, temos $\left[a_{0}, \mathcal{A}\right]=0$, ou seja, $a_{0} \in Z(\mathcal{A})$. Em particular, se $\left[\mathcal{A}_{0}, a_{1}\right]=0$, então, $\mathcal{A}_{0} \subseteq Z(\mathcal{A})$, de onde segue $\left[\mathcal{A}_{0}, \mathcal{A}_{1}\right]=0$. Logo, por (1), concluímos que $\mathcal{A}$ é comutativa.

(3) Para cada $x_{0} \in \mathcal{A}_{0}$ e $x_{1} \in \mathcal{A}_{1}$, temos $\left[a_{1}, x_{0}\right] x_{1}=a_{1} x_{0} x_{1}-x_{0} a_{1} x_{1}=a_{1} x_{0} x_{1}-x_{0} x_{1} a_{1}=$ $\left[a_{1}, x_{0} x_{1}\right]=0$, pois $x_{0} x_{1} \in \mathcal{A}_{1}$. Desse modo, $\left[a_{1}, x_{0}\right] \mathcal{A}_{1}\left[a_{1}, x_{0}\right]=0$. Assim, como $\left[a_{1}, x_{0}\right] \in \mathcal{A}_{1}$, por (1) do Lema 1.2 .5 , vale $\left[a_{1}, x_{0}\right]=0$, para todo $x_{0} \in \mathcal{A}_{0}$, ou seja, $\left[a_{1}, \mathcal{A}_{0}\right]=0$. Logo, o resultado segue de (2).

Lema 1.2.7. Seja $\mathcal{A}=\mathcal{A}_{0} \oplus \mathcal{A}_{1}$ uma superálgebra semiprima associativa. Se $a \in \mathcal{A}_{1}$ é tal que $a x_{1}, x_{1} a \in Z\left(\mathcal{A}_{0}\right)$, para todo $x_{1} \in \mathcal{A}_{1}$, então, $a^{2} \in Z(\mathcal{A})$. 
Demonstração. Se $a \in \mathcal{A}_{1}$, então, $a^{2} \in \mathcal{A}_{0}$ e por hipótese, temos

$$
\begin{gathered}
0=\left[a^{2}, a x_{1}\right]=a^{3} x_{1}-a x_{1} a^{2}=a\left[a^{2}, x_{1}\right] \mathrm{e} \\
0=\left[a^{2}, x_{1} a\right]=a^{2} x_{1} a-x_{1} a^{3}=\left[a^{2}, x_{1}\right] a,
\end{gathered}
$$

para todo $x_{1} \in \mathcal{A}_{1}$. Multiplicando a primeira igualdade à esquerda por $a$, a segunda à direita por $a$, e subtraindo uma da outra, obtemos $a^{2}\left[a^{2}, x_{1}\right]-\left[a^{2}, x_{1}\right] a^{2}=0$, para todo $x_{1} \in \mathcal{A}_{1}$, isto é, $\left[a^{2},\left[a^{2}, \mathcal{A}_{1}\right]\right]=0$. Tomando $x_{1}=a$, decorre $a^{2} \in Z\left(\mathcal{A}_{0}\right)$, e portanto, $\left[a^{2},\left[a^{2}, \mathcal{A}_{0}\right]\right]=0$. Logo, $\left[a^{2},\left[a^{2}, \mathcal{A}\right]\right]=0$. Agora observe que, pela demonstração do Lema 1.2.4, o fato de $\mathcal{A}$ ser superálgebra semiprima associativa, implica em $\mathcal{A}$ ser uma álgebra semiprima associativa. Aplicando, então, o Lema 1.1.7, concluímos $a^{2} \in Z(\mathcal{A})$.

O próximo lema é uma generalização do Lema 1.2.5.

Lema 1.2.8. Sejam $\mathcal{A}$ uma superálgebra associativa semiprima e $U$ um ideal graduado de $\mathcal{A}$. São válidas as seguintes afirmações:

(i) Se $u_{1} \in U_{1}$ é tal que $u_{1} U_{1} u_{1}=0$, então, $u_{1}=0$;

(ii) Se $u_{0} \in U_{0}$ e $u_{1} \in U_{1}$ são tais que $u_{0} U_{i} u_{1}=u_{1} U_{i} u_{0}=0$, onde $i=0$ ou $i=1$, então, $u_{0} U u_{1}=u_{1} U u_{0}=0$

(iii) Se $u_{1} \in U_{1}$ é tal que $u_{1} U_{0}=0$ ou $U_{0} u_{1}=0$, então, $u_{1}=0$.

Demonstração. (i) Inicialmente, observe que as inclusões

$$
\begin{aligned}
u_{1} U u_{1} U u_{1} & \subseteq u_{1}\left(U_{0} u_{1} U_{0}\right) u_{1}+u_{1} U_{0} u_{1} U_{1} u_{1}+u_{1} U_{1} u_{1} U_{0} u_{1}+u_{1} U_{1} u_{1} U_{1} u_{1} \\
& \subseteq u_{1} U_{1} u_{1}+\left(u_{1} U_{1} u_{1}\right) U u_{1}+u_{1} U\left(u_{1} U_{1} u_{1}\right)=0
\end{aligned}
$$

implicam $u_{1} U u_{1} U u_{1}=0$. Assim, considerando $I=\operatorname{ideal}\left\langle u_{1}\right\rangle$, o ideal de $\mathcal{A}$ gerado por $u_{1}$, temos $I^{5}=0$. Portanto, $I=0$, pois $\mathcal{A}$ é semiprima; o que implica $u_{1}=0$.

(ii) Afirmamos que $U_{0}$ é uma álgebra semiprima. Com efeito, sabemos que $\mathcal{A}_{0}$ é uma álgebra semiprima, pelo Lema 1.2.4. Além disso, se $I$ é um ideal de $U_{0}$ tal que $I^{2}=0$, então, considerando $J=$ ideal $\langle I\rangle$, o ideal de $\mathcal{A}_{0}$ gerado por $I$, é possível garantir $J^{3}=0$, o que implica $J=0$ e $I=0$. Se $u_{0} U_{0} u_{1}=u_{1} U_{0} u_{0}=0$, então, $\left(u_{0} U_{1} u_{1}\right) U_{0}\left(u_{0} U_{1} u_{1}\right)=u_{0} U_{1}\left(u_{1} U_{0} u_{0}\right) U_{1} u_{1}=0$. Desse modo, $u_{0} U_{1} u_{1}=0$, pois $u_{0} U_{1} u_{1} \subseteq U_{0}$ e $U_{0}$ é uma álgebra semiprima. Portanto, $u_{0} U u_{1}=0$. Analogamente, $\left(u_{1} U_{1} u_{0}\right) U_{0}\left(u_{1} U_{1} u_{0}\right)=0$ implica $u_{1} U_{1} u_{0}=0$, e então, $u_{1} U u_{0}=0$. Agora, supondo $u_{0} U_{1} u_{1}=u_{1} U_{1} u_{0}=0$, temos $\left(u_{0} U_{0} u_{1}\right) U_{1}\left(u_{0} U_{0} u_{1}\right)=u_{0} U_{0}\left(u_{1} U_{1} u_{0}\right) U_{0} u_{1}=0$. Assim, como $u_{0} U_{0} u_{1} \subseteq U_{1}$, por (i), concluímos $u_{0} U_{0} u_{1}=0$. Portanto, $u_{0} U u_{1}=0$. Do mesmo modo, $\left(u_{1} U_{0} u_{0}\right) U_{1}\left(u_{1} U_{0} u_{0}\right)=0$ implica $u_{1} U_{0} u_{0}=0$. Logo, $u_{1} U u_{0}=0$.

(iii) Se $u_{1} U_{0}=0$, então, $u_{1} U_{0}\left(x_{1} u_{1}\right)=\left(x_{1} u_{1}\right) U_{0} u_{1}=0$, para todo $x_{1} \in \mathcal{A}_{1}$. Assim, como $x_{1} u_{1} \in$ $U \cap \mathcal{A}_{0}=U_{0}$ e $u_{1} \in U_{1}$, temos $u_{1} U\left(x_{1} u_{1}\right)=0$, por (ii). Multiplicando esta última igualdade à esquerda por $x_{1}$, obtemos $\left(x_{1} u_{1}\right) U\left(x_{1} u_{1}\right)=0$. Desse modo, $x_{1} u_{1}=0$, para todo $x_{1} \in \mathcal{A}_{1}$, pois $\mathcal{A}$ é semiprima, isto é, $\mathcal{A}_{1} u_{1}=0$. Portanto, $u_{1} \mathcal{A}_{1} u_{1}=0$. Logo, por (i), no caso particular $U=\mathcal{A}$, concluímos $\mathcal{A}_{1} \ni u_{1}=0$. Finalmente, supondo $U_{0} u_{1}=0$, pelos mesmos raciocínios 
temos $u_{1} U_{0}\left(u_{1} x_{1}\right)=\left(u_{1} x_{1}\right) U_{0} u_{1}=0$, para todo $x_{1} \in \mathcal{A}_{1}$, e consequentemente $\left(u_{1} x_{1}\right) U u_{1}=0$. Multiplicando esta última igualdade à direita por $x_{1}$, temos $\left(u_{1} x_{1}\right) U\left(u_{1} x_{1}\right)=0$, o que implica $u_{1} x_{1}=0$, para todo $x_{1} \in \mathcal{A}_{1}$, isto é, $u_{1} \mathcal{A}_{1}=0$. Portanto, $u_{1} \mathcal{A}_{1} u_{1}=0$, e assim, $u_{1}=0$.

Lema 1.2.9. Sejam $\mathcal{A}$ uma superálgebra associativa semiprima e $U$ um ideal graduado de $\mathcal{A}$. Se $a_{1} \in \mathcal{A}_{1}$ satisfaz $\left[U_{0} a_{1}, U_{0}\right]=0$, então, $U_{0} a_{1} \subseteq Z(\mathcal{A})$.

Demonstração. Para todos $u_{0}, v_{0} \in U_{0}$ e $x_{0} \in \mathcal{A}_{0}$, por hipótese temos

$$
\begin{aligned}
0 & =\left[u_{0} a_{1}, x_{0} v_{0}\right]=u_{0} a_{1} x_{0} v_{0}-x_{0} v_{0} u_{0} a_{1} \\
& =u_{0} a_{1} x_{0} v_{0}-x_{0} u_{0} a_{1} v_{0}+x_{0} u_{0} a_{1} v_{0}-x_{0} v_{0} u_{0} a_{1} \\
& =\left[u_{0} a_{1}, x_{0}\right] v_{0}+x_{0}\left[u_{0} a_{1}, v_{0}\right] \\
& =\left[u_{0} a_{1}, x_{0}\right] v_{0}
\end{aligned}
$$

pois $x_{0} v_{0} \in U \cap \mathcal{A}_{0}=U_{0}$, isto é, $\left[u_{0} a_{1}, x_{0}\right] U_{0}=0$. Assim, como $\left[u_{0} a_{1}, x_{0}\right] \in U_{1}$, por (iii) do Lema 1.2.8, temos $\left[u_{0} a_{1}, x_{0}\right]=0$, para todo $x_{0} \in \mathcal{A}_{0}$, ou seja, $\left[u_{0} a_{1}, \mathcal{A}_{0}\right]=0$. Desse modo, $0=$ $\left[u_{0} a_{1},\left(u_{0} a_{1}\right) x_{1}\right]=u_{0} a_{1}\left[u_{0} a_{1}, x_{1}\right]$ e $0=\left[u_{0} a_{1}, x_{1}\left(u_{0} a_{1}\right)\right]=\left[u_{0} a_{1}, x_{1}\right] u_{0} a_{1}$, para quaisquer $u_{0} \in U_{0}$ e $x_{1} \in \mathcal{A}_{1}$. Subtraindo estas duas últimas igualdades, obtemos $u_{0} a_{1}\left[u_{0} a_{1}, x_{1}\right]-\left[u_{0} a_{1}, x_{1}\right] u_{0} a_{1}=$ $\left[u_{0} a_{1},\left[u_{0} a_{1}, x_{1}\right]\right]=0$, para todo $x_{1} \in \mathcal{A}_{1}$. Portanto, como $\left[u_{0} a_{1},\left[u_{0} a_{1}, x_{0}\right]\right]=\left[u_{0} a_{1}, 0\right]=0$, para todo $x_{0} \in \mathcal{A}_{0}$, concluímos $\left[u_{0} a_{1},\left[u_{0} a_{1}, \mathcal{A}\right]\right]=0$, para todo $u_{0} \in U_{0}$. Finalmente, vale $u_{0} a_{1} \in Z(\mathcal{A})$, para todo $u_{0} \in U_{0}$, pelo Lema 1.1 .7 , pois $\mathcal{A}$ é uma álgebra semiprima.

A seguir, $\left[\mathcal{A}_{0}, \mathcal{A}_{0}\right]$ é o subgrupo aditivo de $\mathcal{A}$ gerado pelos elementos da forma $\left[x_{0}, y_{0}\right]=x_{0} y_{0}-$ $y_{0} x_{0}$, onde $x_{0}, y_{0} \in \mathcal{A}_{0}$, e definimos $U$ como sendo o ideal de $\mathcal{A}$ gerado por $\left[\mathcal{A}_{0}, \mathcal{A}_{0}\right]$. Observe que $U$ é um ideal graduado de $\mathcal{A}$, e vale o próximo resultado.

Lema 1.2.10. Se $\mathcal{A}=\mathcal{A}_{0} \oplus \mathcal{A}_{1}$ é superálgebra associativa semiprima, $a_{1} \in \mathcal{A}_{1}$ é um elemento tal que $U_{0} a_{1} \subseteq Z(\mathcal{A})$ e $a_{1}\left[U_{0}, U_{0}\right]=0$, então, $a_{1} U=0$.

Demonstração. Pelas hipóteses, temos

$$
0=x\left(u_{0} a_{1}\right)\left[v_{0}, w_{0}\right]=u_{0} a_{1} x\left[v_{0}, w_{0}\right]
$$

para todos $u_{0}, v_{0}, w_{0} \in U_{0}$ e $x \in \mathcal{A}$, ou seja, $U_{0} a_{1} \mathcal{A}\left[U_{0}, U_{0}\right]=0$. Em particular, $U_{0} a_{1} \mathcal{A}_{0}\left[U_{0}, U_{0}\right]=0$ e $a_{1} \mathcal{A}_{0}\left[U_{0}, U_{0}\right] \subseteq U_{1}$ implicam $a_{1} \mathcal{A}_{0}\left[U_{0}, U_{0}\right]=0$, por (iii) do Lema 1.2.8; e $U_{0} a_{1} \mathcal{A}_{1}\left[U_{0}, U_{0}\right]=0$ implica $\left(a_{1} \mathcal{A}_{1}\left[U_{0}, U_{0}\right]\right) U_{0}\left(a_{1} \mathcal{A}_{1}\left[U_{0}, U_{0}\right]\right)=0$, e consequentemente, $a_{1} \mathcal{A}_{1}\left[U_{0}, U_{0}\right]=0$, pois $a_{1} \mathcal{A}_{1}\left[U_{0}, U_{0}\right] \subseteq U_{0}$ e, como vimos anteriormente, $U_{0}$ é álgebra semiprima. Portanto, $a_{1} \mathcal{A}\left[U_{0}, U_{0}\right]=0$. A partir deste fato, obtemos

$$
\begin{aligned}
0 & =a_{1} y\left[u_{0}, x_{0} v_{0}\right] \\
& =a_{1} y u_{0} x_{0} v_{0}-a_{1} y x_{0} v_{0} u_{0} \\
& =a_{1} y u_{0} x_{0} v_{0}-a_{1} y x_{0} u_{0} v_{0}+a_{1} y x_{0} u_{0} v_{0}-a_{1} y x_{0} v_{0} u_{0} \\
& =a_{1} y\left[u_{0}, x_{0}\right] v_{0}+a_{1}\left(y x_{0}\right)\left[u_{0}, v_{0}\right] \\
& =a_{1} y\left[u_{0}, x_{0}\right] v_{0},
\end{aligned}
$$

para quaisquer $y \in \mathcal{A}, x_{0} \in \mathcal{A}_{0}$ e $u_{0}, v_{0} \in U_{0}$, isto é, $\left(a_{1} \mathcal{A}\left[U_{0}, \mathcal{A}_{0}\right]\right) U_{0}=0$. Em particular, $\left(a_{1} \mathcal{A}_{0}\left[U_{0}, \mathcal{A}_{0}\right]\right) U_{0}=0$ e $a_{1} \mathcal{A}_{0}\left[U_{0}, \mathcal{A}_{0}\right] \subseteq U_{1}$ implicam $a_{1} \mathcal{A}_{0}\left[U_{0}, \mathcal{A}_{0}\right]=0$; e de $\left(a_{1} \mathcal{A}_{1}\left[U_{0}, \mathcal{A}_{0}\right]\right) U_{0}=0$ 
e $a_{1} \mathcal{A}_{1}\left[U_{0}, \mathcal{A}_{0}\right] \subseteq U_{0}$ segue $a_{1} \mathcal{A}_{1}\left[U_{0}, \mathcal{A}_{0}\right]=0$, pelo Lema 1.2 .8 (iii) e pela semiprimalidade de $U_{0}$. Portanto, $a_{1} \mathcal{A}\left[U_{0}, \mathcal{A}_{0}\right]=0$. Usando os mesmos raciocínios, temos

$$
\begin{aligned}
0 & =a_{1} y\left[z_{0}, x_{0} v_{0}\right] \\
& =a_{1} y z_{0} x_{0} v_{0}-a_{1} y x_{0} v_{0} z_{0} \\
& =a_{1} y z_{0} x_{0} v_{0}-a_{1} y x_{0} z_{0} v_{0}+a_{1} y x_{0} z_{0} v_{0}-a_{1} y x_{0} v_{0} z_{0} \\
& =a_{1} y\left[z_{0}, x_{0}\right] v_{0}-a_{1}\left(y x_{0}\right)\left[v_{0}, z_{0}\right] \\
& =a_{1} y\left[z_{0}, x_{0}\right] v_{0},
\end{aligned}
$$

para todos $y \in \mathcal{A}, x_{0}, z_{0} \in \mathcal{A}_{0}$ e $v_{0} \in U_{0}$, isto é, $\left(a_{1} \mathcal{A}\left[\mathcal{A}_{0}, \mathcal{A}_{0}\right]\right) U_{0}=0$. Assim, $\left(a_{1} \mathcal{A}_{0}\left[\mathcal{A}_{0}, \mathcal{A}_{0}\right]\right) U_{0}=0 \mathrm{e}$ $a_{1} \mathcal{A}_{0}\left[\mathcal{A}_{0}, \mathcal{A}_{0}\right] \subseteq U_{1}$ implicam $a_{1} \mathcal{A}_{0}\left[\mathcal{A}_{0}, \mathcal{A}_{0}\right]=0$ e $\left(a_{1} \mathcal{A}_{1}\left[\mathcal{A}_{0}, \mathcal{A}_{0}\right]\right) U_{0}=0$ e $a_{1} \mathcal{A}_{1}\left[\mathcal{A}_{0}, \mathcal{A}_{0}\right] \subseteq U_{0}$ implicam $a_{1} \mathcal{A}_{1}\left[\mathcal{A}_{0}, \mathcal{A}_{0}\right]=0$, pelo Lema 1.2 .8 (iii) e por $U_{0}$ ser semiprima. Desse modo, $a_{1} \mathcal{A}\left[\mathcal{A}_{0}, \mathcal{A}_{0}\right]=0$, o que implica $a_{1} U=0$, pela definição de $U$.

Lema 1.2.11. Sejam $\mathcal{A}=\mathcal{A}_{0} \oplus \mathcal{A}_{1}$ uma superálgebra associativa semiprima e $a_{0}, b_{0} \in \mathcal{A}_{0}$ tais que $a_{0}\left[\mathcal{A}_{0}, b_{0}\right]=0$. Então, $\left[a_{0}, b_{0}\right]=0$.

Demonstração. Para quaisquer $x_{0}, y_{0} \in \mathcal{A}_{0}$, temos

$$
\begin{aligned}
0 & =a_{0}\left[x_{0} y_{0}, b_{0}\right] \\
& =a_{0} x_{0} y_{0} b_{0}-a_{0} b_{0} x_{0} y_{0} \\
& =a_{0} x_{0} y_{0} b_{0}-a_{0} x_{0} b_{0} y_{0}+a_{0} x_{0} b_{0} y_{0}-a_{0} b_{0} x_{0} y_{0} \\
& =a_{0} x_{0}\left[y_{0}, b_{0}\right]+a_{0}\left[x_{0}, b_{0}\right] y_{0} \\
& =a_{0} x_{0}\left[y_{0}, b_{0}\right] .
\end{aligned}
$$

Desse modo, para cada $x_{0} \in \mathcal{A}_{0}$, temos $\left[a_{0}, b_{0}\right] x_{0}\left[a_{0}, b_{0}\right]=a_{0}\left(b_{0} x_{0}\right)\left[a_{0}, b_{0}\right]-b_{0} a_{0} x_{0}\left[a_{0}, b_{0}\right]=0$, o que nos permite concluir $\left[a_{0}, b_{0}\right]=0$, pois $\mathcal{A}_{0}$ é uma álgebra semiprima.

\section{3 Álgebra de Grassmann, superidentidades e superálgebras de Jordan}

Definimos a álgebra de Grassmann $\Gamma$ como a álgebra associativa gerada pelo conjunto

$$
\left\{1, e_{1}, e_{2}, \ldots, e_{n}, \ldots \mid e_{i} e_{j}=-e_{j} e_{i}\right\}
$$

sobre um anel $\Phi$ de característica diferente de 2 . Uma base para esta álgebra é dada por

$$
\left\{1, e_{i_{1}} e_{i_{2}} \cdots e_{i_{k}} \mid i_{1}<i_{2}<\ldots<i_{k}, k \geq 1\right\}
$$

Podemos dotar a álgebra de Grassmann com uma estrutura de superálgebra definindo uma $\mathbb{Z}_{2}$-graduação da seguinte maneira: $\Gamma=\Gamma_{0} \oplus \Gamma_{1}$, onde $\Gamma_{0}$ é o subespaço gerado por produtos de comprimento par, e $\Gamma_{1}$ é o subespaço gerado por produtos de comprimento ímpar.

Observe que pela definição do produto de $\Gamma$, temos que os elementos de $\Gamma_{0}$ comutam entre si e comutam com elementos de $\Gamma_{1}$, enquanto que os elementos de $\Gamma_{1}$ anticomutam entre si, isto é, se $x_{i} \in \Gamma_{i}, y_{j} \in \Gamma_{j}$, então, $x_{i} y_{j}=(-1)^{i j} y_{j} x_{i}$, onde $i, j \in\{0,1\}$. 
Seja $\mathcal{A}=\mathcal{A}_{0} \oplus \mathcal{A}_{1}$ uma superálgebra e considere a $\Phi$-álgebra $\Gamma \otimes \mathcal{A}$. Definimos a envolvente de Grassmann de $\mathcal{A}$ como sendo a subálgebra $\Gamma(\mathcal{A})=\Gamma_{0} \otimes \mathcal{A}_{0}+\Gamma_{1} \otimes \mathcal{A}_{1}$ de $\Gamma \otimes \mathcal{A}$. Agora, se $\mathcal{M}$ é uma variedade de álgebras sobre $\Phi$ (por exemplo, a variedade das álgebras associativas, ou comutativas, ou das álgebras de Jordan), dizemos que a superálgebra $\mathcal{A}$ pertence a $\mathcal{M}$ (ou $\mathcal{A}$ é uma $\mathcal{M}$-superálgebra) se $\Gamma(\mathcal{A}) \in \mathcal{M}$.

Exemplo 1.3.1. Vamos determinar quando uma superálgebra $\mathcal{A}$ é uma superálgebra associativa. Sejam $a_{i} \in \Gamma_{i}, b_{j} \in \Gamma_{j}, c_{k} \in \Gamma_{k}, x_{i} \in \mathcal{A}_{i}, y_{j} \in \mathcal{A}_{j}$ e $w_{k} \in \mathcal{A}_{k}, i, j, k \in\{0,1\}$. Para que a envolvente de Grassmann de $\mathcal{A}$ seja uma álgebra associativa devemos ter

$$
\left[\left(a_{i} \otimes x_{i}\right)\left(b_{j} \otimes y_{j}\right)\right]\left(c_{k} \otimes w_{k}\right)-\left(a_{i} \otimes x_{i}\right)\left[\left(b_{j} \otimes y_{j}\right)\left(c_{k} \otimes w_{k}\right)\right]=0,
$$

isto é,

$$
\left(\left(a_{i} b_{j}\right) c_{k}\right) \otimes\left(\left(x_{i} y_{j}\right) w_{k}\right)-\left(a_{i}\left(b_{j} c_{k}\right)\right) \otimes\left(x_{i}\left(y_{j} w_{k}\right)\right)=0,
$$

Portanto, $\left(a_{i} b_{j} c_{k}\right) \otimes\left(\left(x_{i} y_{j}\right) w_{k}-x_{i}\left(y_{j} w_{k}\right)\right)=0$. Para que isso aconteça, basta que a igualdade $\left(x_{i} y_{j}\right) w_{k}=x_{i}\left(y_{j} w_{k}\right)$ seja válida, o que quer dizer que para que $\mathcal{A}$ seja uma superálgebra associativa, basta que ela seja associativa como álgebra.

Exemplo 1.3.2. De modo análogo, podemos determinar quando uma superálgebra $\mathcal{A}$ é uma superálgebra comutativa. Sejam $a_{i} \in \Gamma_{i}, b_{j} \in \Gamma_{j}, x_{i} \in \mathcal{A}_{i}$ e $y_{j} \in \mathcal{A}_{j}, i, j \in\{0,1\}$. Para que a envolvente de Grassmann de $\mathcal{A}$ seja uma álgebra comutativa devemos ter

$$
\begin{gathered}
\left(a_{i} \otimes x_{i}\right)\left(b_{j} \otimes y_{j}\right)-\left(b_{j} \otimes y_{j}\right)\left(a_{i} \otimes x_{i}\right)=0 \\
\left(a_{i} b_{j}\right) \otimes\left(x_{i} y_{j}\right)-\left(a_{i} b_{j}\right) \otimes\left((-1)^{i j} y_{j} x_{i}\right)=0 \\
\left(a_{i} b_{j}\right) \otimes\left(x_{i} y_{j}-(-1)^{i j} y_{j} x_{i}\right)=0 .
\end{gathered}
$$

Para tanto, basta que ocorra $x_{i} y_{j}=(-1)^{i j} y_{j} x_{i}$. Quando isso acontece, dizemos que a álgebra é supercomutativa.

Dizemos que as igualdades dos exemplos anteriores, que envolvem apenas elementos homogêneos, são superidentidades.

Define-se álgebra de Jordan como sendo uma álgebra comutativa que satisfaz $x^{2}(y x)=\left(x^{2} y\right) x$ (identidade de Jordan). Dada uma álgebra associativa $A$, é fácil verificar que introduzindo o produto de Jordan em $A$, ela se torna uma álgebra de Jordan com esse produto.

Desse modo, uma superálgebra $\mathcal{A}$ é uma superálgebra de Jordan se a envolvente de Grassmann de $\mathcal{A}, \Gamma(\mathcal{A})$, é uma álgebra de Jordan. Para tanto, basta que sejam satisfeitas as seguintes superidentidades:

(1) Para quaisquer $x_{i} \in \mathcal{A}_{i}, y_{j} \in \mathcal{A}_{j}$,

$$
x_{i} y_{j}-(-1)^{i j} y_{j} x_{i}=0
$$


(2) Para todos $x_{i} \in \mathcal{A}_{i}, y_{j} \in \mathcal{A}_{j}, z_{k} \in \mathcal{A}_{k}, w_{l} \in \mathcal{A}_{l}$,

$$
\begin{aligned}
0= & \left(x_{i} y_{j}\right)\left(z_{k} w_{l}\right)+(-1)^{i(j+k+l)+(j+k) l}\left(w_{l} y_{j}\right)\left(z_{k} x_{i}\right)+(-1)^{i j}\left(y_{j} x_{i}\right)\left(z_{k} w_{l}\right) \\
& +(-1)^{i(j+k+l)+k l}\left(y_{j} w_{l}\right)\left(z_{k} x_{i}\right)+(-1)^{j(k+l)+k l}\left(x_{i} w_{l}\right)\left(z_{k} y_{j}\right) \\
& +(-1)^{j(k+l)+k(l+i)}\left(w_{l} x_{i}\right)\left(z_{k} y_{j}\right)-(-1)^{j(k+l)+k l}\left(\left(x_{i} w_{l}\right) z_{k}\right) y_{j} \\
& -(-1)^{j(k+l)+k(l+i)}\left(\left(w_{l} x_{i}\right) z_{k}\right) y_{j}-\left(\left(x_{i} y_{j}\right) z_{k}\right) w_{l}-(-1)^{i(j+k+l)+(k+j) l}\left(\left(w_{l} y_{j}\right) z_{k}\right) x_{i} \\
& -(-1)^{i j}\left(\left(y_{j} x_{i}\right) z_{k}\right) w_{l}-(-1)^{i(j+k+l)+k l}\left(\left(y_{j} w_{l}\right) z_{k}\right) x_{i}
\end{aligned}
$$

Observe que para obter a última superidentidade, precisamos fazer a linearização completa da identidade de Jordan, uma vez que na álgebra de Grassmann o quadrado de qualquer elemento gerador é nulo.

\subsection{Adjunção de identidades}

Seja $\Phi$ um anel com elemento identidade 1. Dada uma álgebra arbitrária $A$ sobre $\Phi$, podemos construir uma nova álgebra $A^{\#}$ sobre $\Phi$ com identidade, pelo processo de adjunção de identidades. O processo consiste em considerar $\Phi$ como um módulo sobre si mesmo, e assim, 1 é um gerador de $\Phi$, isto é, $\Phi=\Phi \cdot 1$. Desse modo, podemos considerar $A^{\#}=A \oplus \Phi \cdot 1$, onde a multiplicação de $A^{\#}$ é dada por

$$
(a, \alpha)(b, \beta)=(a b+\alpha b+\beta a, \alpha \beta),
$$

para todos $(a, \alpha),(b, \beta) \in A^{\#}$. Dizemos que $A^{\#}$ é a álgebra obtida pela adjunção formal de um elemento identidade à álgebra $A$. De fato, temos que para todo $(a, \alpha) \in A^{\#}$, valem $(0,1)(a, \alpha)=$ $(0 a+\alpha 0+1 a, 1 \alpha)=(a, \alpha)$ e $(a, \alpha)(0,1)=(a 0+1 a+\alpha 0, \alpha 1)=(a, \alpha)$.

Exemplo 1.4.1. Seja $A$ o ideal dos elementos de termo constante 0 na álgebra não comutativa livre gerada por $x$ e $y$ sobre um corpo $F$. Defina $R=A \oplus A \oplus F$, a álgebra obtida pela adjunção formal de um elemento identidade à álgebra $A \oplus A$. Afirmamos que $R$ é um anel semiprimo. De fato, seja $(a, b, \lambda) \in R$ um elemento tal que $(a, b, \lambda) R(a, b, \lambda)=0$, então, em particular, para $(0,0,1) \in R$, temos

$$
\begin{aligned}
0 & =(a, b, \lambda)(0,0,1)(a, b, \lambda) \\
& =(a, b, \lambda)(a, b, \lambda) \\
& =\left(a^{2}+2 \lambda a, b^{2}+2 \lambda b, \lambda^{2}\right) .
\end{aligned}
$$

Assim, concluímos diretamente que vale $\lambda=0$, pois $F$ é corpo, e isso implica $a^{2}=b^{2}=0$. Portanto, $a=b=0$, pois $a, b \in A$ são somas de produtos arbitrários de $x$ e $y$ com coeficientes em $F$. Desse modo, $(a, b, \lambda)=(0,0,0)$. Além disso, o único elemento idempotente não nulo de $R$ é $(0,0,1)$. Com efeito, se $0 \neq(a, b, \lambda) \in R$ é tal que $(a, b, \lambda)^{2}=(a, b, \lambda)$, então, $\left(a^{2}+2 \lambda a, b^{2}+2 \lambda b, \lambda^{2}\right)=(a, b, \lambda)$, de onde vemos $\lambda=0$ ou $\lambda=1$. Entretanto, se $\lambda=0$, então, $a^{2}=a$ e $b^{2}=b$, o que implica $a=b=0$, pois as palavras $a^{2}$ e $b^{2}$ possuem o dobro do tamanho das palavras $a$ e $b$, respectivamente. Assim, $(a, b, \lambda)=0$, o que é uma contradição. Portanto, $\lambda=1$ e temos $a^{2}=-a$ e $b^{2}=-b$, o que nos permite concluir $a=b=0$. Logo, $(a, b, \lambda)=(0,0,1)$. 


\section{Capítulo 2}

\section{Derivações e superderivações de Jordan em anéis e superálgebras primas}

\subsection{Derivações de Jordan em anéis primos}

O objetivo desta seção é demonstrar o famoso resultado de I. N. Herstein [16] que afirma que toda derivação de Jordan em um anel primo de característica diferente de 2 é uma derivação. Recorde que estamos considerando apenas anéis associativos. Iniciamos com algumas definições.

Definição 2.1.1. Uma derivação em um anel $A$ é uma aplicação aditiva $D: A \rightarrow A$ tal que

$$
D(a b)=D(a) b+a D(b)
$$

para todos $a, b \in A$.

Exemplo 2.1.1. Pela demonstração do Lema 1.1.7, se $A$ é um anel e $a$ é um elemento arbitrário de $A$, sabemos que a aplicação $D: A \rightarrow A$, definida por $D(x)=x a-a x$, para todo $x \in A$, é uma derivação.

Dado um anel associativo $A$, podemos construir um novo anel, chamado de anel de Jordan de $A$, redefinindo o produto por $a \circ b=a b+b a$, para todos $a, b \in A$, onde $a b$ é o produto de $a$ por $b$ no anel $A$.

Definição 2.1.2. Uma derivação de Jordan em um anel $A$ é uma derivação no anel de Jordan de A.

Assim, se $D: A \rightarrow A$ é uma derivação de Jordan, então, $D$ é aditiva e satisfaz

$$
D(a \circ b)=D(a) \circ b+a \circ D(b)
$$

para quaisquer $a, b \in A$, isto é,

$$
D(a b+b a)=D(a) b+b D(a)+a D(b)+D(b) a .
$$

Se $A$ é um anel de característica diferente de 2, podemos redefinir uma derivação de Jordan 
como sendo uma aplicação aditiva que satisfaz

$$
D\left(a^{2}\right)=D(a) a+a D(a)
$$

para todo $a \in A$. De fato, linearizando (2.2) obtemos (2.1); e reciprocamente, escolhendo $b=a$ em (2.1), concluímos (2.2), pois $A$ tem característica diferente de 2 . Note também que toda derivação é uma derivação de Jordan, mas a recíproca nem sempre é verdadeira.

Exemplo 2.1.2. Seja $A$ um anel com característica diferente de 2, e tal que uma das seguintes condições é satisfeita:

(1) $A$ contém um elemento $x$ tal que $a x a=0$, para todo $a \in A$, mas $c x d \neq 0$, para alguns elementos $c, d \in A$;

(2) $a^{2}=0$, para todo $a \in A$, mas existem elementos $e, f \in A$ tais que $e f \neq 0$.

No primeiro caso, a aplicação $D: A \rightarrow A$, definida por $D(a)=a x$, para todo $a \in A$, é uma derivação de Jordan. De fato, claramente é aditiva e

$$
\begin{aligned}
D(a \circ b)-D(a) \circ b-a \circ D(b) & =(a \circ b) x-(a x) \circ b-a \circ(b x) \\
& =a b x+b a x-a x b-b a x-a b x-b x a \\
& =-(b x a+a x b)=-(a+b) x(a+b)=0 .
\end{aligned}
$$

Porém, $D$ não é uma derivação. Com efeito,

$$
D(c d)-D(c) d-c D(d)=(c d) x-(c x) d-c(d x)=-c x d \neq 0
$$

No segundo caso, toda aplicação aditiva de $A$ nele mesmo é uma derivação de Jordan, já que o produto de Jordan é nulo, pois $(a+b)^{2}=0$ implica $a \circ b=a b+b a=0$. Entretanto, a função identidade, por exemplo, não é uma derivação, pois ef $\neq 0$. Um exemplo de anel do segundo tipo é a álgebra de Banach obtida de $\mathbb{R}^{3}$ com a norma euclidiana, definindo o produto de $a$ e $b$ em $\mathbb{R}^{3}$ como sendo o seu produto vetorial, $a \times b$, projetado sobre um dos eixos coordenados.

Proposição 2.1.1. Sejam $A$ um anel com característica diferente de 2 e $D$ uma derivação de Jordan de $A$. Então, as seguintes afirmações são válidas:

(1) $D(a b a)=D(a) b a+a D(b) a+a b D(a)$, para todos $a, b \in A$

(2) $D(a b c+c b a)=D(a) b c+a D(b) c+a b D(c)+D(c) b a+c D(b) a+c b D(a)$, para quaisquer $a, b, c \in A$.

Demonstração. (1) Considere o elemento $B=D(a(a b+b a)+(a b+b a) a)$. Por um lado, temos

$$
\begin{aligned}
B= & D(a)(a b+b a)+(a b+b a) D(a)+a D(a b+b a)+D(a b+b a) a \\
= & D(a)(a b+b a)+(a b+b a) D(a)+a(D(a) b+b D(a)+a D(b) \\
& +D(b) a)+(D(a) b+b D(a)+a D(b)+D(b) a) a \\
= & D(a) a b+2 D(a) b a+2 a b D(a)+b a D(a)+a D(a) b+a^{2} D(b) \\
& +2 a D(b) a+b D(a) a+D(b) a^{2},
\end{aligned}
$$


Por outro lado, sabemos

$$
\begin{aligned}
B & =D\left(\left(a^{2} b+b a^{2}\right)+2 a b a\right) \\
& =D\left(a^{2} b+b a^{2}\right)+2 D(a b a) \\
& =D\left(a^{2}\right) b+b D\left(a^{2}\right)+D(b) a^{2}+a^{2} D(b)+2 D(a b a) \\
& =D(a) a b+a D(a) b+b D(a) a+b a D(a)+D(b) a^{2}+a^{2} D(b)+2 D(a b a) .
\end{aligned}
$$

Comparando as duas expressões obtidas, como a característica de $A$ é diferente de 2 , concluímos o desejado.

(2) Basta linearizar a igualdade do enunciado do item (1).

Dada uma derivação de Jordan $D$, denotaremos $a^{b}=D(a b)-D(a) b-a D(b)$, para quaisquer $a, b \in A$. Observe que ocorre $a^{b}=0$, para todos $a, b \in A$, se, e somente se, $D$ é uma derivação em $A$. Portanto, nosso objetivo será provar esta última igualdade. Adotando essa notação, temos as seguintes propriedades.

- Para quaisquer $a, b \in A$, temos

$$
\begin{aligned}
a^{b}+b^{a} & =D(a b)-D(a) b-a D(b)+D(b a)-D(b) a-b D(a) \\
& =D(a b+b a)-D(a) b-b D(a)-a D(b)-D(b) a=0,
\end{aligned}
$$

pois $D$ é uma derivação de Jordan. Portanto, $a^{b}=-b^{a}$.

- Para quaisquer $a, b, c \in A$, sabemos

$$
\begin{aligned}
a^{b+c} & =D(a(b+c))-D(a)(b+c)-a D(b+c) \\
& =D(a b)-D(a) b-a D(b)+D(a c)-D(a) c-a D(c) \\
& =a^{b}+a^{c} .
\end{aligned}
$$

Teorema 2.1.1. (Cusack, [9]) Sejam $A$ um anel de característica diferente de 2 e $D: A \rightarrow A$ uma derivação de Jordan. Para quaisquer $a, b, r \in A$, temos

$$
a^{b} r(a b-b a)+(a b-b a) r a^{b}=0
$$

Demonstração. Seja $B=a b r b a+b a r a b$. Por (1) da Proposição 2.1.1, temos

$$
\begin{aligned}
D(B)= & D(a(b r b) a)+D(b(a r a) b) \\
= & D(a) b r b a+a D(b r b) a+a b r b D(a)+D(b) a r a b+b D(a r a) b+b a r a D(b) \\
= & D(a) b r b a+a D(b) r b a+a b D(r) b a+a b r D(b) a+a b r b D(a) \\
& +D(b) a r a b+b D(a) r a b+b a D(r) a b+b a r D(a) b+b a r a D(b),
\end{aligned}
$$


e por (2) da Proposição 2.1.1, segue

$$
\begin{aligned}
D(B) & =D((a b) r(b a)+(b a) r(a b)) \\
& =D(a b) r b a+a b D(r) b a+a b r D(b a)+D(b a) r a b+b a D(r) a b+b a r D(a b) .
\end{aligned}
$$

Comparando estas duas expressões, obtemos

$$
\begin{aligned}
a^{b} r b a+b^{a} r a b & =-a b r b^{a}-b a r a^{b} \\
a^{b} r(b a-a b) & =(a b-b a) r a^{b} \\
a^{b} r(a b-b a) & =-(a b-b a) r a^{b} .
\end{aligned}
$$

Desse modo, concluímos que a igualdade $a^{b} r(a b-b a)+(a b-b a) r a^{b}=0$ é válida.

Os resultados obtidos nos permitem mostrar o seguinte teorema.

Teorema 2.1.2. (Herstein, [16]) Se $A$ é um anel primo de característica diferente de 2 , e $D: A \rightarrow A$ é uma derivação de Jordan, então, $D$ é uma derivação em $A$.

Demonstração. Fixemos $a$ e $b$ elementos de $A$. Se $a b \neq b a$, então, $a b-b a \neq 0$, e consequentemente, por (2.3) e pela Proposição 1.1.3, temos $a^{b}=0$. Agora, suponhamos $a b=b a$. Se ambos $a$ e $b$ pertencem a $Z(A)$, então, pela definição de derivação de Jordan, seguem as igualdades

$$
\begin{aligned}
D(a b+b a) & =D(a) b+b D(a)+a D(b)+D(b) a \\
2 D(a b) & =2(D(a) b+a D(b)) \\
D(a b) & =D(a) b+a D(b),
\end{aligned}
$$

pois a característica de $A$ é diferente de 2 . Portanto, $a^{b}=0$. Por fim, suponhamos, sem perda de generalidade, que $a$ não pertence a $Z(A)$, isto é, existe $c \in A$ tal que $a c \neq c a$. Portanto $a(b+c) \neq$ $(b+c) a$, o que implica $a^{c}=0$ e $a^{b+c}=0$. Logo,

$$
a^{b}=a^{b}+0=a^{b}+a^{c}=a^{b+c}=0 .
$$

O caso em que $b$ não pertence a $Z(A)$ é análogo.

\subsection{Superderivações de Jordan em superálgebras primas}

Nesta seção apresentamos a generalização do resultado da seção anterior desse capítulo de anéis para superálgebras. Iniciamos generalizando as definições de derivação e derivação de Jordan para superálgebras. O sentido das definições a seguir é justificado pela relação entre superálgebras e a álgebra de Grassmann. Estes resultados estão contidos em [10].

Definição 2.2.1. Sejam $\mathcal{A}$ uma superálgebra e $D: \mathcal{A} \rightarrow \mathcal{A}$ uma aplicação $\Phi$-linear. Dizemos que $D$ é uma superderivação de grau $i$, onde $i=0,1$, se $D\left(\mathcal{A}_{j}\right) \subseteq \mathcal{A}_{j+i(\bmod 2)}$, onde $j=0,1$, e

$$
D(x y)=D(x) y+(-1)^{i|x|} x D(y) \text {, para quaisquer } x, y \in \mathcal{A}_{0} \cup \mathcal{A}_{1} .
$$


Uma superderivação é a soma de uma superderivação de grau 0 com uma superderivação de grau 1.

Dada uma superálgebra $\mathcal{A}$, introduzindo um novo produto definido por

$$
x \circ_{s} y=\frac{1}{2}\left(x y+(-1)^{|x||y|} y x\right), \text { para todos } x, y \in \mathcal{A}_{0} \cup \mathcal{A}_{1},
$$

$\mathcal{A}$ torna-se uma superálgebra de Jordan, o que motiva a seguinte

Definição 2.2.2. Se $D: \mathcal{A} \rightarrow \mathcal{A}$ é uma aplicação $\Phi$-linear, então, $D$ é dita uma superderivação de Jordan de grau $i$, onde $i=0,1$, se $D\left(\mathcal{A}_{j}\right) \subseteq \mathcal{A}_{j+i(\bmod 2)}$, onde $j=0,1$, e

$$
D\left(x \circ_{s} y\right)=D(x) \circ_{s} y+(-1)^{i|x|} x \circ_{s} D(y), \text { para todos } x, y \in \mathcal{A}_{0} \cup \mathcal{A}_{1} .
$$

Uma superderivação de Jordan é a soma de uma superderivação de Jordan de grau 0 com uma superderivação de grau 1.

Note que se a superálgebra for trivial ou se nos restringirmos à parte par da superálgebra, os conceitos de superderivação e de superderivação de Jordan coincidem com os de derivação e de derivação de Jordan, respectivamente.

Lema 2.2.1. Seja $\mathcal{A}$ uma superálgebra. Se $D: \mathcal{A} \rightarrow \mathcal{A}$ é uma superderivação, então, $D$ é uma superderivação de Jordan.

Demonstração. Seja $D=D_{0}+D_{1}$, com $D_{i}$ uma superderivação de grau $i, i=0,1$. Vamos provar que $D_{i}$ é uma superderivação de Jordan de grau $i$. Já sabemos que a inclusão $D\left(\mathcal{A}_{j}\right) \subseteq \mathcal{A}_{i+j(\bmod 2)}$, onde $j=0,1$, é válida. Assim, dados $x, y \in \mathcal{A}_{0} \cup \mathcal{A}_{1}$, temos

$$
\begin{aligned}
D_{i}\left(x \circ_{s} y\right) & =D_{i}\left(\frac{1}{2}\left(x y+(-1)^{|x||y|} y x\right)\right) \\
& =\frac{1}{2}\left(D_{i}(x y)+(-1)^{|x||y|} D_{i}(y x)\right) \\
& =\frac{1}{2}\left(D_{i}(x) y+(-1)^{i|x|} x D_{i}(y)+\left(-1^{|x||y|} D_{i}(y) x+(-1)^{|x||y|+i|y|} y D_{i}(x)\right)\right) \\
& =\frac{1}{2}\left(D_{i}(x) y+(-1)^{(|x|+i)|y|} y D_{i}(x)+(-1)^{i|x|} x D_{i}(y)+(-1)^{|x||y|} D_{i}(y) x\right) .
\end{aligned}
$$

Agora, observe que as igualdades $\left|D_{i}(x)\right|=|x|+i$ e $i|x|+|x|\left|D_{i}(y)\right|=|x|(i+i+|y|)=|x||y|$ implicam

$$
\begin{aligned}
D_{i}\left(x \circ_{s} y\right) & =\frac{1}{2}\left(D_{i}(x) y+(-1)^{\left|D_{i}(x)\right||y|} y D_{i}(x)\right)+(-1)^{i|x|} \frac{1}{2}\left(x D_{i}(y)+(-1)^{|x|\left|D_{i}(y)\right|} D_{i}(y) x\right) \\
& =D_{i}(x) \circ_{s} y+(-1)^{i|x|} x \circ_{s} D_{i}(y) .
\end{aligned}
$$

A recíproca deste lema não é verdadeira, como poderá ser visto nos Exemplos 2.2.1, 2.2.2 e 2.2.3. Todavia, sob determinadas condições, a recíproca é verdadeira. De fato, o resultado que estende o caso de anéis (álgebras) é o seguinte: "toda superderivação de Jordan em uma superálgebra associativa prima, cuja parte par é não comutativa, é uma superderivação". Para provar tal resultado serão necessários alguns lemas.

Denotaremos aqui o comutador de dois elementos $x, y \in A$ por $[x, y]=x y-y x$, e o supercomutador por $[x, y]_{s}=x y-(-1)^{|x||y|} y x$. 
Lema 2.2.2. Seja $\mathcal{A}=\mathcal{A}_{0} \oplus \mathcal{A}_{1}$ uma superálgebra. Se $D$ é uma superderivação de Jordan em $\mathcal{A}$, então,

$$
D\left(\left[x_{1}^{2}, y\right]\right)=\left[\left[D\left(x_{1}\right), x_{1}\right]_{s}, y\right]_{s}+\left[x_{1}^{2}, D(y)\right],
$$

para todos $x_{1} \in \mathcal{A}_{1}, y \in \mathcal{A}$.

Demonstração. Sejam $x_{1} \in \mathcal{A}_{1}$ e $y=y_{0}+y_{1} \in \mathcal{A}$. Observe que as seguintes igualdades são válidas

$$
\begin{aligned}
4 x_{1} \circ_{s}\left(x_{1} \circ_{s} y\right) & =4 x_{1} \circ_{s}\left(x_{1} \circ_{s} y_{0}+x_{1} \circ_{s} y_{1}\right) \\
& =2 x_{1} \circ_{s}\left(x_{1} y_{0}+y_{0} x_{1}+x_{1} y_{1}-y_{1} x_{1}\right) \\
& =2\left(x_{1} \circ_{s}\left(x_{1} y_{0}\right)+x_{1} \circ_{s}\left(y_{0} x_{1}\right)+x_{1} \circ_{s}\left(x_{1} y_{1}\right)-x_{1} \circ_{s}\left(y_{1} x_{1}\right)\right) \\
& =x_{1}^{2} y_{0}-x_{1} y_{0} x_{1}+x_{1} y_{0} x_{1}-y_{0} x_{1}^{2}+x_{1}^{2} y_{1}+x_{1} y_{1} x_{1}-x_{1} y_{1} x_{1}-y_{1} x_{1}^{2} \\
& =x_{1}^{2} y_{0}-y_{0} x_{1}^{2}+x_{1}^{2} y_{1}-y_{1} x_{1}^{2} \\
& =x_{1}^{2} y-y x_{1}^{2}=\left[x_{1}^{2}, y\right] .
\end{aligned}
$$

Além disso, $D=D_{0}+D_{1}$ tal que $D_{i}$ é uma superderivação de Jordan de grau $i$ de $\mathcal{A}$, onde $i=0,1$. Desse modo, vamos provar que $D_{0}$ satisfaz a equação (2.4). Assim, temos

$$
\begin{aligned}
D_{0}\left(\left[x_{1}^{2}, y\right]\right)= & D_{0}\left(4 x_{1} \circ_{s}\left(x_{1} \circ_{s} y\right)\right) \\
= & 4 D_{0}\left(x_{1} \circ_{s}\left(x_{1} \circ_{s} y_{0}\right)\right)+4 D_{0}\left(x_{1} \circ_{s}\left(x_{1} \circ_{s} y_{1}\right)\right) \\
= & 4 D_{0}\left(x_{1}\right) \circ_{s}\left(x_{1} \circ_{s} y_{0}\right)+4 x_{1} \circ_{s} D_{0}\left(x_{1} \circ_{s} y_{0}\right)+4 D_{0}\left(x_{1}\right) \circ_{s}\left(x_{1} \circ_{s} y_{1}\right) \\
& +4 x_{1} \circ_{s} D_{0}\left(x_{1} \circ_{s} y_{1}\right),
\end{aligned}
$$

e calculando cada somando separadamente, obtemos

- $4 D_{0}\left(x_{1}\right) \circ_{s}\left(x_{1} \circ_{s} y_{0}\right)=2 D_{0}\left(x_{1}\right) \circ_{s}\left(x_{1} y_{0}+y_{0} x_{1}\right)$

$$
=D_{0}\left(x_{1}\right) x_{1} y_{0}-x_{1} y_{0} D_{0}\left(x_{1}\right)+D_{0}\left(x_{1}\right) y_{0} x_{1}-y_{0} x_{1} D_{0}\left(x_{1}\right) ;
$$

- $4 x_{1} \circ_{s} D_{0}\left(x_{1} \circ_{s} y_{0}\right)=4 x_{1} \circ_{s}\left(D_{0}\left(x_{1}\right) \circ_{s} y_{0}+x_{1} \circ_{s} D_{0}\left(y_{0}\right)\right)$

$$
\begin{aligned}
= & 2 x_{1} \circ_{s}\left(D_{0}\left(x_{1}\right) y_{0}+y_{0} D_{0}\left(x_{1}\right)+x_{1} D_{0}\left(y_{0}\right)+D_{0}\left(y_{0}\right) x_{1}\right) \\
= & x_{1} D_{0}\left(x_{1}\right) y_{0}-D_{0}\left(x_{1}\right) y_{0} x_{1}+x_{1} y_{0} D_{0}\left(x_{1}\right)-y_{0} D_{0}\left(x_{1}\right) x_{1} \\
& +x_{1}^{2} D_{0}\left(y_{0}\right)-D_{0}\left(y_{0}\right) x_{1}^{2} ;
\end{aligned}
$$

- $4 D_{0}\left(x_{1}\right) \circ_{s}\left(x_{1} \circ_{s} y_{1}\right)=2 D_{0}\left(x_{1}\right) \circ_{s}\left(x_{1} y_{1}-y_{1} x_{1}\right)$

$$
=D_{0}\left(x_{1}\right) x_{1} y_{1}+x_{1} y_{1} D_{0}\left(x_{1}\right)-D_{0}\left(x_{1}\right) y_{1} x_{1}-y_{1} x_{1} D_{0}\left(x_{1}\right) ;
$$

- $4 x_{1} \circ_{s} D_{0}\left(x_{1} \circ_{s} y_{1}\right)=4 x_{1} \circ_{s}\left(D_{0}\left(x_{1}\right) \circ_{s} y_{1}+x_{1} \circ_{s} D_{0}\left(y_{1}\right)\right)$

$$
\begin{aligned}
= & 2 x_{1} \circ_{s}\left(D_{0}\left(x_{1}\right) y_{1}-y_{1} D_{0}\left(x_{1}\right)+x_{1} D_{0}\left(y_{1}\right)-D_{0}\left(y_{1}\right) x_{1}\right) \\
= & x_{1} D_{0}\left(x_{1}\right) y_{1}+D_{0}\left(x_{1}\right) y_{1} x_{1}-x_{1} y_{1} D_{0}\left(x_{1}\right)-y_{1} D_{0}\left(x_{1}\right) x_{1} \\
& +x_{1}^{2} D_{0}\left(y_{1}\right)-D_{0}\left(y_{1}\right) x_{1}^{2} .
\end{aligned}
$$


Consequentemente, a substituição de cada somando na igualdade original resulta em

$$
\begin{aligned}
D_{0}\left(\left[x_{1}^{2}, y\right]\right)= & \left(D_{0}\left(x_{1}\right) x_{1}+x_{1} D_{0}\left(x_{1}\right)\right) y_{0}-y_{0}\left(D_{0}\left(x_{1}\right) x_{1}+x_{1} D_{0}\left(x_{1}\right)\right)+\left(D_{0}\left(x_{1}\right) x_{1}+x_{1} D_{0}\left(x_{1}\right)\right) y_{1} \\
& -y_{1}\left(D_{0}\left(x_{1}\right) x_{1}+x_{1} D_{0}\left(x_{1}\right)\right)+x_{1}^{2} D_{0}\left(y_{0}+y_{1}\right)-D_{0}\left(y_{0}+y_{1}\right) x_{1}^{2} \\
= & {\left[\left[D_{0}\left(x_{1}\right), x_{1}\right]_{s}, y_{0}\right]_{s}+\left[\left[D_{0}\left(x_{1}\right), x_{1}\right]_{s}, y_{1}\right]_{s}+\left[x_{1}^{2}, D_{0}(y)\right] } \\
= & {\left[\left[D_{0}\left(x_{1}\right), x_{1}\right]_{s}, y\right]_{s}+\left[x_{1}^{2}, D_{0}(y)\right] . }
\end{aligned}
$$

Analogamente, pode-se provar que $D_{1}$ satisfaz a equação (2.4), e portanto, $D$ também a satisfaz.

Lema 2.2.3. Seja $D$ uma superderivação de Jordan de grau $i$, onde $i=0,1$, em uma superálgebra $\mathcal{A}=\mathcal{A}_{0} \oplus \mathcal{A}_{1}$. Defina uma aplicação $\delta: \mathcal{A} \times \mathcal{A} \rightarrow \mathcal{A}$ por

$$
\delta(x, y)=D(x y)-D(x) y-(-1)^{i|x|} x D(y), \text { para todos } x, y \in \mathcal{A}_{0} \cup \mathcal{A}_{1} .
$$

Então, $\delta$ é biaditiva e

(i) $\delta\left(x_{0}, y_{1}\right)=-\delta\left(y_{1}, x_{0}\right)$, para todos $x_{0} \in \mathcal{A}_{0}$ e $y_{1} \in \mathcal{A}_{1}$;

(ii) $\delta\left(x_{1}, y_{1}\right)=\delta\left(y_{1}, x_{1}\right)$, para quaisquer $x_{1}, y_{1} \in \mathcal{A}_{1}$.

Além disso, supondo que vale $D\left(x_{0} y_{0}\right)=D\left(x_{0}\right) y_{0}+x_{0} D\left(y_{0}\right)$, para todos $x_{0}, y_{0} \in \mathcal{A}_{0}$, temos:

(iii) $\left[\delta\left(\mathcal{A}_{1}, \mathcal{A}_{1}\right), \mathcal{A}_{0}\right]=0$;

(iv) $x_{0} \delta\left(x_{1}, y_{1}\right)=\delta\left(x_{0} x_{1}, y_{1}\right)+\delta\left(x_{0}, x_{1}\right) y_{1}$, para quaisquer $x_{0} \in \mathcal{A}_{0}, x_{1}, y_{1} \in \mathcal{A}_{1}$;

(v) $\delta\left(x_{1}, y_{1}\right) x_{0}=\delta\left(x_{1} x_{0}, y_{1}\right)-(-1)^{i} y_{1} \delta\left(x_{0}, x_{1}\right)$, para todos $x_{0} \in \mathcal{A}_{0}, x_{1}, y_{1} \in \mathcal{A}_{1}$;

(vi) $\delta\left(x_{0}, \mathcal{A}_{1}\right) \mathcal{A}_{1}\left[\mathcal{A}_{0}, x_{0}\right]=0$, para qualquer $x_{0} \in \mathcal{A}_{0}$;

(vii) $\left[\mathcal{A}_{0}, x_{0}\right] \mathcal{A}_{1} \delta\left(x_{0}, \mathcal{A}_{1}\right)=0$, para todo $x_{0} \in \mathcal{A}_{0}$.

Demonstração. Dados $x, z \in \mathcal{A}_{j}$ e $y, w \in \mathcal{A}_{k}$, onde $j, k=0,1$, temos

$$
\text { - } \begin{aligned}
\delta(x+z, y) & =D(x y+z y)-D(x) y-D(z) y-(-1)^{i|x+z|}(x+z) D(y) \\
& =D(x y)-D(x) y-(-1)^{i|x|} x D(y)+D(z y)-D(z) y-(-1)^{i|z|} z D(y) \\
& =\delta(x, y)+\delta(z, y),
\end{aligned}
$$

pois $x+z \in \mathcal{A}_{j}$ implica $|x+z|=j=|x|=|z|$.

$$
\begin{aligned}
-\delta(x, y+w) & =D(x y+x w)-D(x) y-D(x) w-(-1)^{i|x|} x(D(y)+D(w)) \\
& =D(x y)-D(x) y-(-1)^{i|x|} x D(y)+D(x w)-D(x) w-(-1)^{i|x|} x D(w) \\
& =\delta(x, y)+\delta(x, w),
\end{aligned}
$$

o que implica que $\delta$ é biaditiva.

Agora, sejam $x_{i}, y_{i}, z_{i}$ elementos arbitrários de $\mathcal{A}_{i}$, onde $i=0,1$.

(i) Pelo fato de $D$ ser uma superderivação de Jordan de grau $i$, seguem as igualdades 


$$
\begin{aligned}
0= & D\left(x_{0} \circ_{s} y_{1}\right)-D\left(x_{0}\right) \circ_{s} y_{1}-(-1)^{i\left|x_{0}\right|} x_{0} \circ_{s} D\left(y_{1}\right) \\
= & D\left(x_{0} y_{1}\right)+D\left(y_{1} x_{0}\right)-D\left(x_{0}\right) y_{1}-(-1)^{i\left|y_{1}\right|} y_{1} D\left(x_{0}\right)-(-1)^{i\left|x_{0}\right|} x_{0} D\left(y_{1}\right) \\
& -(-1)^{i\left|x_{0}\right|} D\left(y_{1}\right) x_{0} \\
= & D\left(x_{0} y_{1}\right)-D\left(x_{0}\right) y_{1}-(-1)^{i\left|x_{0}\right|} x_{0} D\left(y_{1}\right)+D\left(y_{1} x_{0}\right)-D\left(y_{1}\right) x_{0}-(-1)^{i\left|y_{1}\right|} y_{1} D\left(x_{0}\right) \\
= & \delta\left(x_{0}, y_{1}\right)+\delta\left(y_{1}, x_{0}\right) .
\end{aligned}
$$

(ii) De modo análogo ao item (i), temos

$$
\begin{aligned}
0= & D\left(x_{1} \circ_{s} y_{1}\right)-D\left(x_{1}\right) \circ_{s} y_{1}-(-1)^{i\left|x_{1}\right|} x_{1} \circ_{s} D\left(y_{1}\right) \\
= & D\left(x_{1} y_{1}\right)-D\left(y_{1} x_{1}\right)-D\left(x_{1}\right) y_{1}-(-1)^{(i+1)\left|y_{1}\right|} y_{1} D\left(x_{1}\right)-(-1)^{i\left|x_{1}\right|} x_{1} D\left(y_{1}\right) \\
& -(-1)^{i\left|x_{1}\right|}(-1)^{(i+1)\left|x_{1}\right|} D\left(y_{1}\right) x_{1} \\
= & D\left(x_{1} y_{1}\right)-D\left(x_{1}\right) y_{1}-(-1)^{i\left|x_{1}\right|} x_{1} D\left(y_{1}\right)-D\left(y_{1} x_{1}\right)+D\left(y_{1}\right) x_{1}+(-1)^{i\left|y_{1}\right|} y_{1} D\left(x_{1}\right) \\
= & \delta\left(x_{1}, y_{1}\right)-\delta\left(y_{1}, x_{1}\right) .
\end{aligned}
$$

(iii) Pela hipótese que $\left.D\right|_{\mathcal{A}_{0}}$ é uma derivação e como $x_{1}^{2} \in \mathcal{A}_{0}$, são válidas

$$
\begin{aligned}
D\left(\left[x_{1}^{2}, x_{0}\right]\right) & =D\left(x_{1}^{2} x_{0}\right)-D\left(x_{0} x_{1}^{2}\right) \\
& =D\left(x_{1}^{2}\right) x_{0}+x_{1}^{2} D\left(x_{0}\right)-D\left(x_{0}\right) x_{1}^{2}-x_{0} D\left(x_{1}^{2}\right) \\
& =\left[D\left(x_{1}^{2}\right), x_{0}\right]+\left[x_{1}^{2}, D\left(x_{0}\right)\right] .
\end{aligned}
$$

Além disso, pelo Lema 2.2.2, também sabemos $D\left(\left[x_{1}^{2}, x_{0}\right]\right)=\left[\left[D\left(x_{1}\right), x_{1}\right]_{s}, x_{0}\right]_{s}+\left[x_{1}^{2}, D\left(x_{0}\right)\right]$, o que implica

$$
\begin{aligned}
0 & =\left[D\left(x_{1}^{2}\right), x_{0}\right]-\left[\left[D\left(x_{1}\right), x_{1}\right]_{s}, x_{0}\right]_{s} \\
& =\left[D\left(x_{1}^{2}\right)-D\left(x_{1}\right) x_{1}+(-1)^{(i+1)\left|x_{1}\right|} x_{1} D\left(x_{1}\right), x_{0}\right] \\
& =\left[D\left(x_{1}^{2}\right)-D\left(x_{1}\right) x_{1}-(-1)^{i\left|x_{1}\right|} x_{1} D\left(x_{1}\right), x_{0}\right] \\
& =\left[\delta\left(x_{1}, x_{1}\right), x_{0}\right] .
\end{aligned}
$$

Linearizando esta última igualdade, obtemos $\left[\delta\left(x_{1}, y_{1}\right), x_{0}\right]+\left[\delta\left(y_{1}, x_{1}\right), x_{0}\right]=0$. Assim, por (ii), concluímos $\delta\left(x_{1}, y_{1}\right)=\delta\left(y_{1}, x_{1}\right)$. Logo, $\left[\delta\left(x_{1}, y_{1}\right), x_{0}\right]=0$, pois $\mathcal{A}$ é uma álgebra sobre um anel $\Phi$ com característica diferente de 2 .

(iv) Considere o elemento $D\left(x_{0} x_{1} y_{1}\right)$. Por um lado, temos

$$
\begin{aligned}
D\left(x_{0}\left(x_{1} y_{1}\right)\right) & =D\left(x_{0}\right) x_{1} y_{1}+x_{0} D\left(x_{1} y_{1}\right) \quad\left(\text { pois } x_{1} y_{1} \in \mathcal{A}_{0}\right) \\
& =D\left(x_{0}\right) x_{1} y_{1}+x_{0}\left(\delta\left(x_{1}, y_{1}\right)+D\left(x_{1}\right) y_{1}+(-1)^{i\left|x_{1}\right|} x_{1} D\left(y_{1}\right)\right) \\
& =D\left(x_{0}\right) x_{1} y_{1}+x_{0} \delta\left(x_{1}, y_{1}\right)+x_{0} D\left(x_{1}\right) y_{1}+(-1)^{i} x_{0} x_{1} D\left(y_{1}\right) .
\end{aligned}
$$


Por outro lado, ocorrem

$$
\begin{aligned}
D\left(\left(x_{0} x_{1}\right) y_{1}\right)= & \delta\left(x_{0} x_{1}, y_{1}\right)+D\left(x_{0} x_{1}\right) y_{1}+(-1)^{i\left|x_{0} x_{1}\right|} x_{0} x_{1} D\left(y_{1}\right) \\
= & \delta\left(x_{0} x_{1}, y_{1}\right)+\left(\delta\left(x_{0}, x_{1}\right)+D\left(x_{0}\right) x_{1}+(-1)^{i\left|x_{0}\right|} x_{0} D\left(x_{1}\right)\right) y_{1}+ \\
& +(-1)^{i} x_{0} x_{1} D\left(y_{1}\right) \\
= & \delta\left(x_{0} x_{1}, y_{1}\right)+\delta\left(x_{0}, x_{1}\right) y_{1}+D\left(x_{0}\right) x_{1} y_{1}+x_{0} D\left(x_{1}\right) y_{1}+(-1)^{i} x_{0} x_{1} D\left(y_{1}\right) .
\end{aligned}
$$

Igualando as duas expressões encontradas, resulta em

$$
x_{0} \delta\left(x_{1}, y_{1}\right)=\delta\left(x_{0} x_{1}, y_{1}\right)+\delta\left(x_{0}, x_{1}\right) y_{1}
$$

(v) De modo análogo ao item (iv), basta considerar o elemento $D\left(y_{1} x_{1} x_{0}\right)$. Com efeito, por um lado, sabemos

$$
\begin{aligned}
D\left(\left(y_{1} x_{1}\right) x_{0}\right) & =D\left(y_{1} x_{1}\right) x_{0}+y_{1} x_{1} D\left(x_{0}\right) \quad\left(\text { pois } y_{1} x_{1} \in \mathcal{A}_{0}\right) \\
& =\left(\delta\left(y_{1}, x_{1}\right)+D\left(y_{1}\right) x_{1}+(-1)^{i\left|y_{1}\right|} y_{1} D\left(x_{1}\right)\right) x_{0}+y_{1} x_{1} D\left(x_{0}\right) \\
& =\delta\left(y_{1}, x_{1}\right) x_{0}+D\left(y_{1}\right) x_{1} x_{0}+(-1)^{i} y_{1} D\left(x_{1}\right) x_{0}+y_{1} x_{1} D\left(x_{0}\right),
\end{aligned}
$$

enquanto que, por outro lado, obtemos

$$
\begin{aligned}
D\left(y_{1}\left(x_{1} x_{0}\right)\right)= & \delta\left(y_{1}, x_{1} x_{0}\right)+D\left(y_{1}\right) x_{1} x_{0}+(-1)^{i\left|y_{1}\right|} y_{1} D\left(x_{1} x_{0}\right) \\
= & \delta\left(y_{1}, x_{1} x_{0}\right)+D\left(y_{1}\right) x_{1} x_{0}+(-1)^{i} y_{1}\left(\delta\left(x_{1}, x_{0}\right)+D\left(x_{1} x_{0}+\right.\right. \\
& \left.+(-1)^{i\left|x_{1}\right|} x_{1} D\left(x_{0}\right)\right) \\
= & \delta\left(y_{1}, x_{1} x_{0}\right)+D\left(y_{1}\right) x_{1} x_{0}+(-1)^{i} y_{1} \delta\left(x_{1}, x_{0}\right)+(-1)^{i} y_{1} D\left(x_{1} x_{0}\right)+ \\
& +y_{1} x_{1} D\left(x_{0}\right) .
\end{aligned}
$$

Novamente, igualando as duas expressões encontradas, segue

$$
\delta\left(y_{1}, x_{1}\right) x_{0}=\delta\left(y_{1}, x_{1} x_{0}\right)+(-1)^{i} y_{1} \delta\left(x_{1}, x_{0}\right)
$$

Finalmente, por (i) e (ii), como $x_{1} x_{0} \in \mathcal{A}_{1}$, concluímos

$$
\delta\left(x_{1}, y_{1}\right) x_{0}=\delta\left(x_{1} x_{0}, y_{1}\right)-(-1)^{i} y_{1} \delta\left(x_{0}, x_{1}\right)
$$

(vi) Em vista do item (iii), sabemos que os elementos da forma $\delta\left(x_{1}, y_{1}\right)$ comutam com $x_{0} \in \mathcal{A}_{0}$, e pelo item (iv), temos $\delta\left(x_{0}, x_{1}\right) y_{1}=x_{0} \delta\left(x_{1}, y_{1}\right)-\delta\left(x_{0} x_{1}, y_{1}\right)$. Desse modo,

$$
\begin{aligned}
{\left[\delta\left(x_{0}, x_{1}\right) y_{1}, x_{0}\right] } & =\delta\left(x_{0}, x_{1}\right) y_{1} x_{0}-x_{0} \delta\left(x_{0}, x_{1}\right) y_{1} \\
& =x_{0} \delta\left(x_{1}, y_{1}\right) x_{0}-\delta\left(x_{0} x_{1}, y_{1}\right) x_{0}-x_{0}^{2} \delta\left(x_{1}, y_{1}\right)+x_{0} \delta\left(x_{0} x_{1}, y_{1}\right) \\
& =x_{0}^{2} \delta\left(x_{1}, y_{1}\right)-x_{0}^{2} \delta\left(x_{1}, y_{1}\right)-\left(\delta\left(x_{0} x_{1}, y_{1}\right) x_{0}-x_{0} \delta\left(x_{0} x_{1}, y_{1}\right)\right) \\
& =-\left[\delta\left(x_{0} x_{1}, y_{1}\right), x_{0}\right]=0
\end{aligned}
$$


pois $x_{0} x_{1} \in \mathcal{A}_{1}$, ou seja, $\left[\delta\left(x_{0}, \mathcal{A}_{1}\right) \mathcal{A}_{1}, x_{0}\right]=0$, para todo $x_{0} \in \mathcal{A}_{0}$. Assim,

$$
\begin{aligned}
\delta\left(x_{0}, x_{1}\right) z_{1}\left[z_{0}, x_{0}\right]= & \delta\left(x_{0}, x_{1}\right) z_{1} z_{0} x_{0}-\delta\left(x_{0}, x_{1}\right) z_{1} x_{0} z_{0} \\
= & \delta\left(x_{0}, x_{1}\right) z_{1} z_{0} x_{0}-x_{0} \delta\left(x_{0}, x_{1}\right) z_{1} z_{0}+x_{0} \delta\left(x_{0}, x_{1}\right) z_{1} z_{0} \\
& -\delta\left(x_{0}, x_{1}\right) z_{1} x_{0} z_{0} \\
= & {\left[\delta\left(x_{0}, x_{1}\right) z_{1} z_{0}, x_{0}\right]-\left[\delta\left(x_{0}, x_{1}\right) z_{1}, x_{0}\right] z_{0}=0, }
\end{aligned}
$$

isto é, $\delta\left(x_{0}, \mathcal{A}_{1}\right) \mathcal{A}_{1}\left[\mathcal{A}_{0}, x_{0}\right]=0$.

(vii) De modo análogo, por (v), sabemos $y_{1} \delta\left(x_{0}, x_{1}\right)=(-1)^{i} \delta\left(x_{1} x_{0}, y_{1}\right)-(-1)^{i} \delta\left(x_{1}, y_{1}\right) x_{0}$, e usando também o resultado de (iii), temos

$$
\begin{aligned}
{\left[y_{1} \delta\left(x_{0}, x_{1}\right), x_{0}\right]=} & y_{1} \delta\left(x_{0}, x_{1}\right) x_{0}-x_{0} y_{1} \delta\left(x_{0}, x_{1}\right) \\
= & (-1)^{i} \delta\left(x_{1} x_{0}, y_{1}\right) x_{0}-(-1)^{i} \delta\left(x_{1}, y_{1}\right) x_{0}^{2}-(-1)^{i} x_{0} \delta\left(x_{1} x_{0}, y_{1}\right) \\
& +(-1)^{i} x_{0} \delta\left(x_{1}, y_{1}\right) x_{0} \\
= & -(-1)^{i}\left(\delta\left(x_{1}, y_{1}\right) x_{0}-x_{0} \delta\left(x_{1}, y_{1}\right)\right) x_{0}+(-1)^{i}\left(\delta\left(x_{1} x_{0}, y_{1}\right) x_{0}\right. \\
& \left.-x_{0} \delta\left(x_{1} x_{0}, y_{1}\right)\right) \\
= & -(-1)^{i}\left[\delta\left(x_{1}, y_{1}\right), x_{0}\right] x_{0}+(-1)^{i}\left[\delta\left(x_{1} x_{0}, y_{1}\right), x_{0}\right]=0,
\end{aligned}
$$

isto é, $\left[\mathcal{A}_{1} \delta\left(x_{0}, \mathcal{A}_{1}\right), x_{0}\right]=0$. Consequentemente, segue que são válidas

$$
\begin{aligned}
{\left[y_{0}, x_{0}\right] x_{1} \delta\left(x_{0}, y_{1}\right)=} & y_{0} x_{0} x_{1} \delta\left(x_{0}, y_{1}\right)-x_{0} y_{0} x_{1} \delta\left(x_{0}, y_{1}\right) \\
= & \left(y_{0} x_{0} x_{1} \delta\left(x_{0}, y_{1}\right)-y_{0} x_{1} \delta\left(x_{0}, y_{1}\right) x_{0}\right)+\left(y_{0} x_{1} \delta\left(x_{0}, y_{1}\right) x_{0}\right. \\
& -x_{0} y_{0} x_{1} \delta\left(x_{0}, y_{1}\right) \\
= & -y_{0}\left[x_{1} \delta\left(x_{0}, y_{1}\right), x_{0}\right]+\left[y_{0} x_{1} \delta\left(x_{0}, y_{1}\right), x_{0}\right]=0 .
\end{aligned}
$$

Logo, $\left[\mathcal{A}_{0}, x_{0}\right] \mathcal{A}_{1} \delta\left(x_{0}, \mathcal{A}_{1}\right)=0$.

Lema 2.2.4. Sejam $\mathcal{A}$ uma álgebra, $\mathcal{B}$ uma subálgebra de $\mathcal{A}, \mathcal{M}$ um $\mathcal{A}$-bimódulo e $D: \mathcal{A} \rightarrow \mathcal{M}$ uma aplicação linear satisfazendo $D(b \circ a)=D(b) \circ a+b \circ D(a)$, para quaisquer $b \in \mathcal{B}$ e $a \in \mathcal{A}$. Então,

$$
\begin{gathered}
{\left[b, b^{\prime}\right] \mathcal{A}\left[b, b^{\prime}\right] \mathcal{A}\left(D\left(b b^{\prime}\right)-D(b) b^{\prime}-b D\left(b^{\prime}\right)\right)=0, \text { para todos } b, b^{\prime} \in \mathcal{B} \text { e }} \\
\left(D\left(b b^{\prime}\right)-D(b) b^{\prime}-b D\left(b^{\prime}\right)\right) \mathcal{A}\left[b, b^{\prime}\right] \mathcal{A}\left[b, b^{\prime}\right]=0, \text { para quaisquer } b, b^{\prime} \in \mathcal{B}
\end{gathered}
$$

Demonstração. Inicialmente, observe que podemos escrever

$$
\begin{aligned}
2 b \circ(b \circ a)-b^{2} \circ a & =b \circ(b a+a b)-\frac{1}{2}\left(b^{2} a+a b^{2}\right) \\
& =\frac{1}{2}\left(b^{2} a+2 b a b+a b^{2}\right)-\frac{1}{2}\left(b^{2} a+a b^{2}\right) \\
& =b a b,
\end{aligned}
$$


para quaisquer $a \in \mathcal{A}, b \in \mathcal{B}$. Assim, usando essa igualdade, temos

$$
\begin{aligned}
D(b a b)= & D(2 b \circ(b \circ a))-D\left(b^{2} \circ a\right) \\
= & 2 D(b) \circ(b \circ a)+2 b \circ(D(b) \circ a+b \circ D(a))-D\left(b^{2}\right) \circ a-b^{2} \circ D(a) \\
= & D(b) \circ(b a+a b)+b \circ(D(b) a+a D(b)+b D(a)+D(a) b)-\frac{1}{2}\left(D\left(b^{2}\right) a+a D\left(b^{2}\right)\right. \\
& \left.+b^{2} D(a)+D(a) b^{2}\right) \\
= & \frac{1}{2}\left(D(b) b a+D(b) a b+b a D(b)+a b D(b)+b D(b) a+b a D(b)+b^{2} D(a)+b D(a) b\right. \\
& \left.+D(b) a b+a D(b) b+b D(a) b+D(a) b^{2}-D\left(b^{2}\right) a-a D\left(b^{2}\right)-b^{2} D(a)-D(a) b^{2}\right) .
\end{aligned}
$$

Agora, escolhendo $a=b$, segue $D(b \circ b)=D(b) \circ b+b \circ D(b)$, o que implica $D\left(b^{2}\right)=D(b) b+b D(b)$. Substituindo na igualdade acima, temos

$$
\begin{aligned}
D(b a b)= & \frac{1}{2}\left(2 D(b) a b+2 b D(a) b+2 b a D(b)+D(b) b a+a b D(b)+b D(b) a+b^{2} D(a)\right. \\
& \left.+a D(b) b+D(a) b^{2}-D(b) b a-b D(b) a-a D(b) b-a b D(b)-b^{2} D(a)-D(a) b^{2}\right) \\
= & D(b) a b+b D(a) b+b a D(b) .
\end{aligned}
$$

Linearizando a igualdade obtida, segue

$$
D\left(b a b^{\prime}+b^{\prime} a b\right)=D(b) a b^{\prime}+D\left(b^{\prime}\right) a b+b D(a) b^{\prime}+b^{\prime} D(a) b+b a D\left(b^{\prime}\right)+b^{\prime} a D(b),
$$

para todos $a \in \mathcal{A}, b, b^{\prime} \in \mathcal{B}$. Prosseguindo, considere o elemento $W=D\left(b b^{\prime} a b^{\prime} b+b^{\prime} b a b b^{\prime}\right)$. Desse modo, são válidas

$$
\begin{aligned}
W= & D\left(b\left(b^{\prime} a b^{\prime}\right) b\right)+D\left(b^{\prime}(b a b) b^{\prime}\right) \\
= & D(b) b^{\prime} a b^{\prime} b+b D\left(b^{\prime}\right) a b^{\prime} b+b b^{\prime} D(a) b^{\prime} b+b b^{\prime} a D\left(b^{\prime}\right) b+b b^{\prime} a b^{\prime} D(b)+D\left(b^{\prime}\right) b a b b^{\prime} \\
& +b^{\prime} D(b) a b b^{\prime}+b^{\prime} b D(a) b b^{\prime}+b^{\prime} b a D(b) b^{\prime}+b^{\prime} b a b D\left(b^{\prime}\right) \\
= & \left(D(b) b^{\prime}+b D\left(b^{\prime}\right)\right) a b^{\prime} b+\left(D\left(b^{\prime}\right) b+b^{\prime} D(b)\right) a b b^{\prime}+b b^{\prime} a\left(b^{\prime} D(b)+D\left(b^{\prime}\right) b\right) \\
& +b^{\prime} b a\left(D(b) b^{\prime}+b D\left(b^{\prime}\right)\right)+b b^{\prime} D(a) b^{\prime} b+b^{\prime} b D(a) b b^{\prime},
\end{aligned}
$$

e também,

$$
\begin{aligned}
W & =D\left(\left(b b^{\prime}\right) a\left(b^{\prime} b\right)+\left(b^{\prime} b\right) a\left(b b^{\prime}\right)\right) \\
& =D\left(b b^{\prime}\right) a b^{\prime} b+b b^{\prime} D(a) b^{\prime} b+b b^{\prime} a D\left(b^{\prime} b\right)+D\left(b^{\prime} b\right) a b b^{\prime}+b^{\prime} b D(a) b b^{\prime}+b^{\prime} b a D\left(b b^{\prime}\right) .
\end{aligned}
$$

Igualando as expressões encontradas, e usando a igualdade

$$
D\left(b b^{\prime}\right)+D\left(b^{\prime} b\right)=D(b) b^{\prime}+b D\left(b^{\prime}\right)+D\left(b^{\prime}\right) b+b^{\prime} D(b),
$$


chegamos a

$$
\begin{aligned}
0= & \left(D\left(b b^{\prime}\right)-D(b) b^{\prime}-b D\left(b^{\prime}\right)\right) a b b^{\prime}+\left(D\left(b^{\prime} b\right)-D\left(b^{\prime}\right) b-b^{\prime} D(b)\right) a b^{\prime} b \\
& +b b^{\prime} a\left(D\left(b b^{\prime}\right)-D(b) b^{\prime}-b D\left(b^{\prime}\right)\right)+b^{\prime} b a\left(D\left(b^{\prime} b\right)-D\left(b^{\prime}\right) b-b^{\prime} D(b)\right) \\
= & \left(D\left(b b^{\prime}\right)-D(b) b^{\prime}-b D\left(b^{\prime}\right)\right) a\left[b, b^{\prime}\right]+\left[b, b^{\prime}\right] a\left(D\left(b b^{\prime}\right)-D(b) b^{\prime}-b D\left(b^{\prime}\right)\right),
\end{aligned}
$$

para todos $a \in \mathcal{A}, b, b^{\prime} \in \mathcal{B}$. Assim, denotando $m=D\left(b b^{\prime}\right)-D(b) b^{\prime}-b D\left(b^{\prime}\right)$ e $c=\left[b, b^{\prime}\right]$, temos $m a c+c a m=0$, isto é, $m a c=-c a m$, para todo $a \in \mathcal{A}$. Além disso, $c a m a^{\prime} c+c a c a^{\prime} m=0$ implica

$$
c a c a^{\prime} m=-c a m a^{\prime} c=m a c a^{\prime} c=m\left(a c a^{\prime}\right) c=-c a c a^{\prime} m .
$$

Portanto, $2 c a c a^{\prime} m=0$ e consequentemente, $c a c a^{\prime} m=0$, para quaisquer $a, a^{\prime} \in \mathcal{A}$, ou seja,

$$
\left[b, b^{\prime}\right] \mathcal{A}\left[b, b^{\prime}\right] \mathcal{A}\left(D\left(b b^{\prime}\right)-D(b) b^{\prime}-b D\left(b^{\prime}\right)\right)=0, \text { para todos } b, b^{\prime} \in \mathcal{B} .
$$

Finalmente, note que também são válidas

$$
m a c a^{\prime} c=-c a m a^{\prime} c=c a c a^{\prime} m=c\left(a c a^{\prime}\right) m=-m a c a^{\prime} c .
$$

isto é, $2 m a c a^{\prime} c=0$, o que implica $m a c a^{\prime} c=0$, para todos $a, a^{\prime} \in \mathcal{A}$. Logo,

$$
\left(D\left(b b^{\prime}\right)-D(b) b^{\prime}-b D\left(b^{\prime}\right)\right) \mathcal{A}\left[b, b^{\prime}\right] \mathcal{A}\left[b, b^{\prime}\right]=0, \text { para quaisquer } b, b^{\prime} \in \mathcal{B}
$$

\subsubsection{Superderivações de Jordan de grau 0}

Nesta seção, vamos nos focar no estudo de superderivações de Jordan de grau 0.

Proposição 2.2.1. Sejam $\mathcal{A}$ uma álgebra e $D: \mathcal{A} \rightarrow \mathcal{A}$ uma aplicação linear. Suponha que exista uma subálgebra $\mathcal{A}_{0}$ de $\mathcal{A}$ tal que as seguintes condições são válidas:

(1) $D\left(x_{0} \circ x\right)=D\left(x_{0}\right) \circ x+x_{0} \circ D(x)$, para todos $x_{0} \in \mathcal{A}_{0}$ e $x \in \mathcal{A}$;

(2) $D\left(\mathcal{A}_{0}\right) \subseteq \mathcal{A}_{0}$

(3) Se $a_{0}, b_{0}, c_{0} \in \mathcal{A}_{0}$ são tais que $a_{0} \mathcal{A} b_{0} \mathcal{A} c_{0}=0$, então, $a_{0}=0$ ou $b_{0}=0$ ou $c_{0}=0$.

Então, $\left.D\right|_{\mathcal{A}_{0}}$ é uma derivação.

Demonstração. Queremos provar que a igualdade $\delta\left(x_{0}, y_{0}\right)=D\left(x_{0} y_{0}\right)-D\left(x_{0}\right) y_{0}-x_{0} D\left(y_{0}\right)=0$ é válida, para quaisquer $x_{0}, y_{0} \in \mathcal{A}_{0}$. Inicialmente, considerando $\mathcal{B}=\mathcal{A}_{0}$ e $\mathcal{M}=\mathcal{A}$ no Lema 2.2.4, temos que vale

$$
\left[x_{0}, y_{0}\right] \mathcal{A}\left[x_{0}, y_{0}\right] \mathcal{A} \delta\left(x_{0}, y_{0}\right)=0
$$

para quaisquer $x_{0}, y_{0} \in \mathcal{A}_{0}$. Assim, como $\left[x_{0}, y_{0}\right] \in \mathcal{A}_{0}$ e $\delta\left(x_{0}, y_{0}\right) \in \mathcal{A}_{0}$, por $(2)$, segue $\left[x_{0}, y_{0}\right]=0$ ou $\delta\left(x_{0}, y_{0}\right)=0$, pelo item (3). Agora, fixamos um elemento $x_{0} \in \mathcal{A}_{0}$ e definimos

$$
A_{1}=\left\{y_{0} \in \mathcal{A}_{0} \mid \delta\left(x_{0}, y_{0}\right)=0\right\} \text { e } A_{2}=\left\{y_{0} \in \mathcal{A}_{0} \mid\left[x_{0}, y_{0}\right]=0\right\} .
$$

Note que $A_{1}$ e $A_{2}$ são subgrupos aditivos de $\mathcal{A}_{0}$ tais que $\mathcal{A}_{0}=A_{1} \cup A_{2}$. Desse modo, como um grupo não pode ser a união de dois subgrupos próprios, concluímos $\mathcal{A}_{0}=A_{1}$ ou $\mathcal{A}_{0}=A_{2}$, isto é, 
$\delta\left(x_{0}, \mathcal{A}_{0}\right)=0$ ou $\left[x_{0}, \mathcal{A}_{0}\right]=0$, para cada $x_{0} \in \mathcal{A}_{0}$. Prosseguindo, considerando os subgrupos

$$
A_{1}^{\prime}=\left\{x_{0} \in \mathcal{A}_{0} \mid \delta\left(x_{0}, \mathcal{A}_{0}\right)=0\right\} \text { e } A_{2}^{\prime}=\left\{x_{0} \in \mathcal{A}_{0} \mid\left[x_{0}, \mathcal{A}_{0}\right]=0\right\},
$$

temos $\mathcal{A}_{0}=A_{1}^{\prime} \cup A_{2}^{\prime}$. Portanto, $\mathcal{A}_{0}=A_{1}^{\prime}$ ou $\mathcal{A}_{0}=A_{2}^{\prime}$, isto é, $\delta\left(\mathcal{A}_{0}, \mathcal{A}_{0}\right)=0$ ou $\mathcal{A}_{0}$ é comutativo. Entretanto, se $\mathcal{A}_{0}$ é comutativo, então, $x_{0} \circ y_{0}=x_{0} y_{0}$, para quaisquer $x_{0}, y_{0} \in \mathcal{A}_{0}$, e concluímos que $D$ restrita a $\mathcal{A}_{0}$ é, de fato, uma derivação.

O próximo teorema nos permitirá concluir que toda superderivação de Jordan de grau 0 em uma superálgebra associativa prima, cuja parte par não é comutativa, é uma superderivação. Uma superderivação de Jordan que não é superderivação é chamada de superderivação de Jordan própria.

Teorema 2.2.1. (Fošner, [10]) Seja $\mathcal{A}=\mathcal{A}_{0} \oplus \mathcal{A}_{1}$ uma superálgebra prima. Suponha que exista uma superderivação de Jordan própria $D: \mathcal{A} \rightarrow \mathcal{A}$ de grau 0. Então, $\mathcal{A}_{0}$ é uma álgebra comutativa e $D\left(\mathcal{A}_{1}\right) \neq 0$.

Demonstração. Inicialmente, observe que se $\mathcal{A}_{0}$ é a parte par de $\mathcal{A}$ e $D$ é uma superderivação de Jordan própria, então, pela Proposição $2.2 .1,\left.D\right|_{\mathcal{A}_{0}}$ é uma derivação. Vejamos que $\mathcal{A}_{0}$ é comutativa. Para cada $x_{0} \in \mathcal{A}_{0}$, como $\delta\left(x_{0}, \mathcal{A}_{1}\right) \subseteq \mathcal{A}_{1}$ e $\left[\mathcal{A}_{0}, x_{0}\right] \subseteq \mathcal{A}_{0}$, por (vi) e (vii) do Lema 2.2.3, temos

$$
\delta\left(x_{0}, \mathcal{A}_{1}\right) \mathcal{A}_{1}\left[\mathcal{A}_{0}, x_{0}\right]=\left[\mathcal{A}_{0}, x_{0}\right] \mathcal{A}_{1} \delta\left(x_{0}, \mathcal{A}_{1}\right)=0 .
$$

Desse modo, pelo Lema 1.2.5, segue $\delta\left(x_{0}, \mathcal{A}_{1}\right)=0$ ou $\left[\mathcal{A}_{0}, x_{0}\right]=0$. Agora, definimos

$$
A_{1}=\left\{x_{0} \in \mathcal{A}_{0} \mid \delta\left(x_{0}, \mathcal{A}_{1}\right)=0\right\} \text { e } A_{2}=\left\{x_{0} \in \mathcal{A}_{0} \mid\left[\mathcal{A}_{0}, x_{0}\right]=0\right\} .
$$

Analogamente ao caso da Proposição 2.2.1, temos $\mathcal{A}_{0}=A_{1} \cup A_{2}$, o que implica que $\mathcal{A}_{0}=A_{1}$ ou $\mathcal{A}_{0}=A_{2}$, isto é, $\delta\left(\mathcal{A}_{0}, \mathcal{A}_{1}\right)=0$ ou $\mathcal{A}_{0}$ é comutativa. Se $\delta\left(\mathcal{A}_{0}, \mathcal{A}_{1}\right)=0$, então, temos $\delta\left(\mathcal{A}_{1}, \mathcal{A}_{0}\right)=0$, por (i) do Lema 2.2.3. Além disso, dados $x_{1}, y_{1} \in \mathcal{A}_{1}$, então, $x_{1}^{2} \in \mathcal{A}_{0}$,

$$
\begin{aligned}
D\left(\left[x_{1}^{2}, y_{1}\right]\right) & =D\left(x_{1}^{2} y_{1}\right)-D\left(y_{1} x_{1}^{2}\right) \\
& =\delta\left(x_{1}^{2}, y_{1}\right)+D\left(x_{1}^{2}\right) y_{1}+x_{1}^{2} D\left(y_{1}\right)-\delta\left(y_{1}, x_{1}^{2}\right)-D\left(y_{1}\right) x_{1}^{2}-y_{1} D\left(x_{1}^{2}\right) \\
& =\left[D\left(x_{1}^{2}\right), y_{1}\right]+\left[x_{1}^{2}, D\left(y_{1}\right)\right],
\end{aligned}
$$

e também,

$$
D\left(\left[x_{1}^{2}, y_{1}\right]\right)=\left[D\left(x_{1}\right) x_{1}+x_{1} D\left(x_{1}\right), y_{1}\right]+\left[x_{1}^{2}, D\left(y_{1}\right)\right]
$$

pelo Lema 2.2.2. Igualando as expressões encontradas, segue $\left[\delta\left(x_{1}, x_{1}\right), y_{1}\right]=0$, para todos $x_{1}, y_{1} \in$ $\mathcal{A}_{1}$. Linearizando esta última igualdade, obtemos

$$
\left[\delta\left(x_{1}, z_{1}\right), y_{1}\right]+\left[\delta\left(z_{1}, x_{1}\right), y_{1}\right]=0
$$

para quaisquer $x_{1}, y_{1}, z_{1} \in \mathcal{A}_{1}$. Assim, como $\delta\left(z_{1}, x_{1}\right)=\delta\left(x_{1}, z_{1}\right)$, por (ii) do Lema 2.2.3, temos $2\left[\delta\left(x_{1}, z_{1}\right), y_{1}\right]=0$, o que implica

$$
\left[\delta\left(x_{1}, z_{1}\right), y_{1}\right]=0
$$

ou seja, $\left[\delta\left(\mathcal{A}_{1}, \mathcal{A}_{1}\right), \mathcal{A}_{1}\right]=0$. Além disso, por (iii) do Lema 2.2 .3 , também vale $\left[\delta\left(\mathcal{A}_{1}, \mathcal{A}_{1}\right), \mathcal{A}_{0}\right]=0$. 
Portanto, $\left[\delta\left(\mathcal{A}_{1}, \mathcal{A}_{1}\right), \mathcal{A}\right]=0$, ou seja, $\delta\left(\mathcal{A}_{1}, \mathcal{A}_{1}\right) \subseteq Z(\mathcal{A})$. Consequentemente, por (v) do Lema 2.2.3, para cada $x_{1}, y_{1} \in \mathcal{A}_{1}$ e $x_{0} \in \mathcal{A}_{0}$, valem

$$
\delta\left(x_{1}, y_{1}\right) x_{0}=\delta\left(x_{1} x_{0}, y_{1}\right)-(-1)^{i} y_{1} \delta\left(x_{0}, x_{1}\right)=\delta\left(x_{1} x_{0}, y_{1}\right) \in Z(\mathcal{A})
$$

pois $\delta\left(\mathcal{A}_{0}, \mathcal{A}_{1}\right)=0$, o que implica $\delta\left(\mathcal{A}_{1}, \mathcal{A}_{1}\right) \mathcal{A}_{0} \subseteq Z(\mathcal{A})$. Desse modo, para cada $x_{1}, y_{1} \in \mathcal{A}_{1}$, $x_{0}, y_{0} \in \mathcal{A}_{0}$ e $a=a_{0}+a_{1} \in \mathcal{A}$, temos

$$
\begin{aligned}
\delta\left(x_{1}, y_{1}\right) a\left[x_{0}, y_{0}\right]= & \delta\left(x_{1}, y_{1}\right) a_{0}\left[x_{0}, y_{0}\right]+\delta\left(x_{1}, y_{1}\right) a_{1}\left[x_{0}, y_{0}\right] \\
= & \delta\left(x_{1}, y_{1}\right) a_{0} x_{0} y_{0}-\delta\left(x_{1}, y_{1}\right) a_{0} y_{0} x_{0}+\delta\left(x_{1}, y_{1}\right) a_{1} x_{0} y_{0} \\
& -\delta\left(x_{1}, y_{1}\right) a_{1} y_{0} x_{0} \\
= & \left(\delta\left(x_{1}, y_{1}\right) a_{0} x_{0} y_{0}-x_{0} \delta\left(x_{1}, y_{1}\right) a_{0} y_{0}\right)+\left(a_{1} \delta\left(x_{1}, y_{1}\right) x_{0} y_{0}\right. \\
& \left.-a_{1} y_{0} \delta\left(x_{1}, y_{1}\right) x_{0}\right) \\
= & {\left[\delta\left(x_{1}, y_{1}\right) a_{0}, x_{0}\right] y_{0}+a_{1}\left[\delta\left(x_{1}, y_{1}\right) x_{0}, y_{0}\right]=0 }
\end{aligned}
$$

ou seja, $\delta\left(x_{1}, y_{1}\right) \mathcal{A}\left[x_{0}, y_{0}\right]=0$. Assim, como $\mathcal{A}$ é uma superálgebra prima e $\delta\left(x_{1}, y_{1}\right),\left[x_{0}, y_{0}\right] \in \mathcal{A}_{0}$ são elementos homogêneos, segue $\delta\left(x_{1}, y_{1}\right)=0$ ou $\left[x_{0}, y_{0}\right]=0$, isto é, $\delta\left(\mathcal{A}_{1}, \mathcal{A}_{1}\right)=0$ ou $\mathcal{A}_{0}$ é comutativa. Entretanto, como também já provamos $\delta\left(\mathcal{A}_{0}, \mathcal{A}_{0}\right)=\delta\left(\mathcal{A}_{0}, \mathcal{A}_{1}\right)=\delta\left(\mathcal{A}_{1}, \mathcal{A}_{0}\right)=0$, não podemos ter $\delta\left(\mathcal{A}_{1}, \mathcal{A}_{1}\right)=0$, pois $D$ é uma superderivação de Jordan própria. Logo, concluímos que $\mathcal{A}_{0}$ é uma álgebra comutativa.

Finalmente, somente falta mostrar $D\left(\mathcal{A}_{1}\right) \neq 0$. Para isso, vamos supor $D\left(\mathcal{A}_{1}\right)=0$. Inicialmente, observe que para $x_{0} \in \mathcal{A}_{0}$ e $x_{1} \in \mathcal{A}_{1}$ elementos quaisquer, temos $x_{0} \circ_{s} x_{1}=\frac{1}{2}\left(x_{0} x_{1}+x_{1} x_{0}\right) \in \mathcal{A}_{1}$. Assim, $0=D\left(x_{0} \circ_{s} x_{1}\right)=D\left(x_{0}\right) \circ_{s} x_{1}+x_{0} \circ_{s} D\left(x_{1}\right)=D\left(x_{0}\right) \circ_{s} x_{1}$, ou seja,

$$
D\left(x_{0}\right) x_{1}+x_{1} D\left(x_{0}\right)=0 .
$$

Em particular, $D\left(x_{0}^{2}\right) x_{1}+x_{1} D\left(x_{0}^{2}\right)=0$. Além disso, como $\left.D\right|_{\mathcal{A}_{0}}$ é uma derivação, também temos $D\left(x_{0}^{2}\right)=2 x_{0} D\left(x_{0}\right)$, pois $\mathcal{A}_{0}$ é comutativa. Substituindo e usando a equação $(2.5)$, seguem as igualdades

$$
\begin{aligned}
0 & =x_{0} D\left(x_{0}\right) x_{1}+x_{1} x_{0} D\left(x_{0}\right) \\
& =-x_{0} x_{1} D\left(x_{0}\right)+x_{1} x_{0} D\left(x_{0}\right) \\
& =\left[x_{1}, x_{0}\right] D\left(x_{0}\right) .
\end{aligned}
$$

Em consequência disso, temos

$$
\begin{aligned}
{\left[x_{1}, x_{0}\right] y_{0} D\left(x_{0}\right) } & =x_{1} x_{0} y_{0} D\left(x_{0}\right)-x_{0} x_{1} y_{0} D\left(x_{0}\right) \\
& =x_{1} y_{0} x_{0} D\left(x_{0}\right)-x_{0} x_{1} y_{0} D\left(x_{0}\right) \\
& =\left[x_{1} y_{0}, x_{0}\right] D\left(x_{0}\right)=0,
\end{aligned}
$$


para qualquer $y_{0} \in \mathcal{A}_{0}$, o que implica $\left[x_{1}, x_{0}\right] \mathcal{A}_{0} D\left(x_{0}\right)=0$ e

$$
\begin{aligned}
{\left[x_{1}, x_{0}\right] y_{1} D\left(x_{0}\right) } & =x_{1} x_{0} y_{1} D\left(x_{0}\right)-x_{0} x_{1} y_{1} D\left(x_{0}\right) \\
& =x_{1} x_{0} y_{1} D\left(x_{0}\right)-\left(x_{1} y_{1}\right) x_{0} D\left(x_{0}\right) \\
& =-x_{1}\left[y_{1}, x_{0}\right] D\left(x_{0}\right)=0,
\end{aligned}
$$

para todo $y_{1} \in \mathcal{A}_{1}$, isto é, $\left[x_{1}, x_{0}\right] \mathcal{A}_{1} D\left(x_{0}\right)=0$. Portanto, $\left[x_{1}, x_{0}\right] \mathcal{A} D\left(x_{0}\right)=0$. Assim, $\left[x_{1}, x_{0}\right]=0$ ou $D\left(x_{0}\right)=0$, para quaisquer $x_{0} \in \mathcal{A}_{0}, x_{1} \in \mathcal{A}_{1}$, pois $\mathcal{A}$ é uma superálgebra prima. $\operatorname{Se} D\left(\mathcal{A}_{0}\right)=0$, então, como estamos supondo $D\left(\mathcal{A}_{1}\right)=0$, teríamos $D=0$, contradizendo o fato de que $D$ é uma superderivaçã de Jordan própria. Entretanto, se $\left[\mathcal{A}_{1}, \mathcal{A}_{0}\right]=0$, então, da igualdade (2.5), temos $D\left(x_{0}\right) x_{1}=0=x_{1} D\left(x_{0}\right)$, para todos $x_{0} \in \mathcal{A}_{0}$ e $x_{1} \in \mathcal{A}_{1}$. Substituindo $x_{1}$ por $y_{0} x_{1}$ e $x_{1} y_{0}$ vale $D\left(x_{0}\right) y_{0} x_{1}=0=x_{1} y_{0} D\left(x_{0}\right)$, ou seja, $D\left(x_{0}\right) \mathcal{A}_{0} x_{1}=0=x_{1} \mathcal{A}_{0} D\left(x_{0}\right)$. Logo, por (ii) do Lema 1.2.5, temos $D\left(x_{0}\right)=0$, para todo $x_{0} \in \mathcal{A}_{0}$, o que nos leva a mesma contradição anterior. Portanto, $D\left(\mathcal{A}_{1}\right) \neq 0$.

A seguir, daremos alguns exemplos de superderivações de Jordan próprias de grau 0, que ilustram o Teorema 2.2.1.

Exemplo 2.2.1. Seja $\mathcal{A}=\mathcal{A}_{0} \oplus \mathcal{A}_{1}$ uma superálgebra prima, comutativa como álgebra e tal que $\mathcal{A}_{1} \neq 0$. Escolha $0 \neq a_{0} \in \mathcal{A}_{0}$ e defina $D: \mathcal{A} \rightarrow \mathcal{A}$ por $D\left(x_{0}+x_{1}\right)=a_{0} x_{1}$, para todos $x_{i} \in \mathcal{A}_{i}$, onde $i=0,1$. Além disso, seja $y_{1} \in \mathcal{A}_{1}$ um elemento tal que $a_{0} y_{1}^{2} \neq 0$. Então, $D$ é uma superderivação de Jordan própria de grau 0 . De fato, $D$ é uma aplicação linear tal que $D\left(\mathcal{A}_{i}\right) \subseteq \mathcal{A}_{i}$, onde $i=0,1$, e para todos $x, y \in \mathcal{A}$, temos

$$
\begin{aligned}
D\left(x \circ_{s} y\right)= & D\left(x_{0} \circ_{s} y_{0}+x_{0} \circ_{s} y_{1}+x_{1} \circ_{s} y_{0}+x_{1} \circ_{s} y_{1}\right) \\
= & \frac{1}{2} D\left(x_{0} y_{0}+y_{0} x_{0}+x_{0} y_{1}+y_{1} x_{0}+x_{1} y_{0}+y_{0} x_{1}+x_{1} y_{1}-y_{1} x_{1}\right) \\
= & \frac{1}{2} a_{0}\left(x_{0} y_{1}+y_{1} x_{0}+x_{1} y_{0}+y_{0} x_{1}\right) \\
= & a_{0} x_{0} y_{1}+a_{0} x_{1} y_{0} \\
= & \frac{1}{2}\left(\left(a_{0} x_{1}\right) y_{0}+y_{0}\left(a_{0} x_{1}\right)+\left(a_{0} x_{1}\right) y_{1}-y_{1}\left(a_{0} x_{1}\right)+x_{0}\left(a_{0} y_{1}\right)+\left(a_{0} y_{1}\right) x_{0}+x_{1}\left(a_{0} y_{1}\right)\right. \\
& \left.-\left(a_{0} y_{1}\right) x_{1}\right) \\
= & \left(a_{0} x_{1}\right) \circ_{s} y_{0}+\left(a_{0} x_{1}\right) \circ_{s} y_{1}+x_{0} \circ_{s}\left(a_{0} y_{1}\right)+x_{1} \circ_{s}\left(a_{0} y_{1}\right) \\
= & \left(a_{0} x_{1}\right) \circ_{s} y+x \circ_{s}\left(a_{0} y_{1}\right) \\
= & D(x) \circ_{s} y+x \circ_{s} D(y) .
\end{aligned}
$$

Contudo, $D\left(y_{1}^{2}\right)=0$ mas $D\left(y_{1}\right) y_{1}+y_{1} D\left(y_{1}\right)=2 a_{0} y_{1}^{2} \neq 0$, isto é, $D$ não é superderivação.

Exemplo 2.2.2. Seja $\mathcal{A}=\mathcal{Q}(\alpha, \beta)$ a superálgebra dos quatérnios, isto é, $\mathcal{A}$ é uma álgebra sobre $\Phi$, de dimensão 4 , com uma base dada por $1, u, v, u v$, e multiplicação definida como segue: $u v=$ $-v u, 0 \neq u^{2}=\alpha \in \Phi$ e $0 \neq v^{2}=\beta \in \Phi$. Uma $\mathbb{Z}_{2}$-graduação para $\mathcal{A}$ pode ser definida por $\mathcal{A}_{0}=\Phi 1+\Phi u v$ e $\mathcal{A}_{1}=\Phi u+\Phi v$. Além disso, consideramos a aplicação $D: \mathcal{A} \rightarrow \mathcal{A}$ dada por $D\left(\lambda_{1} 1+\lambda_{2} u v+\lambda_{3} u+\lambda_{4} v\right)=\lambda_{3} u-\lambda_{4} v$, para todo $\lambda_{i} \in \Phi, i=1,2,3,4$. Então, $D$ é uma 
superderivação de Jordan de grau 0 própria. De fato, se $x=u \in \mathcal{A}$, então, $D\left(x^{2}\right)=D(\alpha)=0$, mas $D(x) x+x D(x)=u^{2}+u^{2}=2 \alpha \neq 0$.

\subsubsection{Superderivações de Jordan de grau 1}

Finalmente, encerramos o capítulo com o estudo das superderivações de Jordan de grau 1.

Proposição 2.2.2. Seja $D: \mathcal{A}_{0} \rightarrow \mathcal{M}$ uma derivação de Jordan, onde $\mathcal{A}_{0}$ é uma álgebra semiprima, que não é comutativa, e $\mathcal{M}$ é um $\mathcal{A}_{0}$-bimódulo. Suponha que as igualdades $a_{0} \mathcal{A}_{0} m=m \mathcal{A}_{0} a_{0}=0$, com $a_{0} \in \mathcal{A}_{0}$ e $m \in \mathcal{M}$, impliquem $a_{0}=0$ ou $m=0$. Então, $D$ é uma derivação.

Demonstração. Primeiramente, observe que escolhendo $\mathcal{A}=\mathcal{B}=\mathcal{A}_{0}$ no Lema 2.2.4, temos

$$
\begin{gathered}
{\left[x_{0}, y_{0}\right] \mathcal{A}_{0}\left[x_{0}, y_{0}\right] \mathcal{A}_{0} \delta\left(x_{0}, y_{0}\right)=0, \text { para todos } x_{0}, y_{0} \in \mathcal{A}_{0} \text { e }} \\
\delta\left(x_{0}, y_{0}\right) \mathcal{A}_{0}\left[x_{0}, y_{0}\right] \mathcal{A}_{0}\left[x_{0}, y_{0}\right]=0, \text { para quaisquer } x_{0}, y_{0} \in \mathcal{A}_{0},
\end{gathered}
$$

onde $\delta\left(x_{0}, y_{0}\right)=D\left(x_{0} y_{0}\right)-D\left(x_{0}\right) y_{0}-x_{0} D\left(y_{0}\right)$. Assim, dados $x_{0}, y_{0} \in \mathcal{A}_{0}$, denotando $a_{0}=\left[x_{0}, y_{0}\right] \in$ $\mathcal{A}_{0}$ e $m=\delta\left(x_{0}, y_{0}\right) \in \mathcal{M}$, segue

$$
\left(a_{0} \mathcal{A}_{0} a_{0}\right) \mathcal{A}_{0} m=0=m \mathcal{A}_{0}\left(a_{0} \mathcal{A}_{0} a_{0}\right),
$$

o que implica, por hipótese, $m=0$ ou $a_{0} \mathcal{A}_{0} a_{0}=0$. Desse modo, como $\mathcal{A}_{0}$ é semiprima, o segundo caso equivale a $a_{0}=0$. Provamos, então, que para quaisquer $x_{0}, y_{0} \in \mathcal{A}_{0}$, vale $\delta\left(x_{0}, y_{0}\right)=0$ ou $\left[x_{0}, y_{0}\right]=0$. Fixando $x_{0} \in \mathcal{A}_{0}$, e definindo

$$
A_{1}=\left\{y_{0} \in \mathcal{A}_{0} \mid \delta\left(x_{0}, y_{0}\right)=0\right\} \text { e } A_{2}=\left\{y_{0} \in \mathcal{A}_{0} \mid\left[x_{0}, y_{0}\right]=0\right\} .
$$

temos que $A_{1}$ e $A_{2}$ são subgrupos aditivos de $\mathcal{A}_{0}$ tais que $\mathcal{A}_{0}=A_{1} \cup A_{2}$, o que implica $\mathcal{A}_{0}=A_{1}$ ou $\mathcal{A}_{0}=A_{2}$, pois um grupo não pode ser união de dois subgrupos próprios. Portanto, $\delta\left(x_{0}, \mathcal{A}_{0}\right)=0$ ou $\left[x_{0}, \mathcal{A}_{0}\right]=0$, para cada $x_{0} \in \mathcal{A}_{0}$. Analogamente, considerando os subgrupos de $\mathcal{A}_{0}$

$$
A_{1}^{\prime}=\left\{x_{0} \in \mathcal{A}_{0} \mid \delta\left(x_{0}, \mathcal{A}_{0}\right)=0\right\} \text { e } A_{2}^{\prime}=\left\{x_{0} \in \mathcal{A}_{0} \mid\left[x_{0}, \mathcal{A}_{0}\right]=0\right\},
$$

temos $\mathcal{A}_{0}=A_{1}^{\prime} \cup A_{2}^{\prime}$, o que implica $\mathcal{A}_{0}=A_{1}^{\prime}$ ou $\mathcal{A}_{0}=A_{2}^{\prime}$. Entretanto, se $\mathcal{A}_{0}=A_{2}^{\prime}$, então, $\left[\mathcal{A}_{0}, \mathcal{A}_{0}\right]=0$, isto é, $\mathcal{A}_{0}$ é comutativo, contradizendo a hipótese. Portanto, $\mathcal{A}_{0}=A_{1}^{\prime}$, ou seja, $\delta\left(\mathcal{A}_{0}, \mathcal{A}_{0}\right)=0$, o que significa que $D$ é uma derivação.

Teorema 2.2.2. (Fošner, [10]) Seja $\mathcal{A}=\mathcal{A}_{0} \oplus \mathcal{A}_{1}$ uma superálgebra associativa prima. Suponha que exista uma superderivação de Jordan pópria $D: \mathcal{A} \rightarrow \mathcal{A}$ de grau 1. Então, $\mathcal{A}$ é uma álgebra comutativa e $D\left(\mathcal{A}_{0}\right) \neq 0$.

Demonstração. Inicialmente, suponhamos por absurdo que $\mathcal{A}_{0}$ não seja comutativa. Desse modo, como $\mathcal{A}$ é superálgebra associativa prima, pelo Lema 1.2.4, $\mathcal{A}_{0}$ é uma álgebra associativa semiprima. Além disso, $D\left(\mathcal{A}_{0}\right) \subseteq \mathcal{A}_{1}$ e $\mathcal{A}_{1}$ é um $\mathcal{A}_{0}$-bimódulo, pela definição de $\mathbb{Z}_{2}$-graduação. Por (ii) do Lema 1.2 .5 , se $a_{0} \in \mathcal{A}_{0}$ e $a_{1} \in \mathcal{A}_{1}$ são elementos tais que $a_{0} \mathcal{A}_{0} a_{1}=a_{1} \mathcal{A}_{0} a_{0}=0$, então, $a_{0}=0$ ou $a_{1}=0$. Assim, $\left.D\right|_{\mathcal{A}_{0}}$ satisfaz as hipóteses da Proposição 2.2.2, pois esta é uma derivação de Jordan, e 
consequentemente, $\left.D\right|_{\mathcal{A}_{0}}$ é uma derivação. Seja $\delta(x, y)=D(x y)-D(x) y-(-1)^{1|x|} x D(y)$, onde $x, y \in \mathcal{A}_{0} \cup \mathcal{A}_{1}$. As seguintes igualdades decorrem do Lema 2.2.3

$$
\begin{aligned}
\delta\left(x_{0} x_{1}, y_{1}\right)+\delta\left(x_{0}, x_{1}\right) y_{1} & =x_{0} \delta\left(x_{1}, y_{1}\right) \quad(\text { por (iv) }) \\
& =\delta\left(x_{1}, y_{1}\right) x_{0} \quad(\text { por (iii) }) \\
& =\delta\left(x_{1} x_{0}, y_{1}\right)+y_{1} \delta\left(x_{0}, x_{1}\right) \quad(\text { por (v)) }
\end{aligned}
$$

Assim, por (iii) do Lema 2.2.3, seguem

$$
\begin{aligned}
{\left[\delta\left(x_{0}, x_{1}\right) y_{1}, y_{0}\right] } & =-\left[\delta\left(x_{0} x_{1}, y_{1}\right), y_{0}\right]+\left[\delta\left(x_{1} x_{0}, y_{1}\right), y_{0}\right]+\left[y_{1} \delta\left(x_{0}, x_{1}\right), y_{0}\right] \\
& =\left[y_{1} \delta\left(x_{0}, x_{1}\right), y_{0}\right] .
\end{aligned}
$$

Multiplicando a igualdade obtida por $z_{1}\left[z_{0}, x_{0}\right]$ à direita, onde $z_{1} \in \mathcal{A}_{1}$ e $x_{0}, z_{0} \in \mathcal{A}_{0}$, temos

$$
\begin{aligned}
{\left[\delta\left(x_{0}, x_{1}\right) y_{1}, y_{0}\right] z_{1}\left[z_{0}, x_{0}\right] } & =\left[y_{1} \delta\left(x_{0}, x_{1}\right), y_{0}\right] z_{1}\left[z_{0}, x_{0}\right] \\
& =y_{1} \delta\left(x_{0}, x_{1}\right)\left(y_{0} z_{1}\right)\left[z_{0}, x_{0}\right]-y_{0} y_{1} \delta\left(x_{0}, x_{1}\right) z_{1}\left[z_{0}, x_{0}\right]=0
\end{aligned}
$$

por (vi) do Lema 2.2.3, para quaisquer $z_{i} \in \mathcal{A}_{i}, i=0,1$, isto é,

$$
\left[\delta\left(x_{0}, x_{1}\right) y_{1}, y_{0}\right] \mathcal{A}_{1}\left[\mathcal{A}_{0}, x_{0}\right]=0 .
$$

Por outro lado, por (vii) do Lema 2.2.3, decorre

$$
\left[z_{0}, x_{0}\right] z_{1}\left[\delta\left(x_{0}, x_{1}\right) y_{1}, y_{0}\right]=\left[z_{0}, x_{0}\right] z_{1} \delta\left(x_{0}, x_{1}\right) y_{1} y_{0}-\left[z_{0}, x_{0}\right] z_{1} y_{0} \delta\left(x_{0}, x_{1}\right) y_{1}=0
$$

para quaisquer $z_{i} \in \mathcal{A}_{i}$, onde $i=0,1$, isto é,

$$
\left[\mathcal{A}_{0}, x_{0}\right] \mathcal{A}_{1}\left[\delta\left(x_{0}, x_{1}\right) y_{1}, y_{0}\right]=0 .
$$

Desse modo, como $\left[\delta\left(x_{0}, x_{1}\right) y_{1}, y_{0}\right] \in \mathcal{A}_{1}$ e $\left[\mathcal{A}_{0}, x_{0}\right] \subseteq \mathcal{A}_{0}$, aplicando (ii) do Lema 1.2.5, segue $\left[\delta\left(x_{0}, x_{1}\right) y_{1}, y_{0}\right]=0$ ou $\left[\mathcal{A}_{0}, x_{0}\right]=0$, para todo $x_{0} \in \mathcal{A}_{0}$. Porém, estamos supondo que $\mathcal{A}_{0}$ não é comutativa, o que implica que ocorre $\left[\delta\left(x_{0}, x_{1}\right) y_{1}, y_{0}\right]=0$. Desta igualdade e dos itens (iii) e (iv) do Lema 2.2.3, temos

$$
\begin{aligned}
0 & =\left[\delta\left(x_{0}, x_{1}\right) y_{1}, y_{0}\right] \\
& =\left[x_{0} \delta\left(x_{1}, y_{1}\right), y_{0}\right]-\left[\delta\left(x_{0} x_{1}, y_{1}\right), y_{0}\right] \\
& =\left[x_{0} \delta\left(x_{1}, y_{1}\right), y_{0}\right] \\
& =\left[\delta\left(x_{1}, y_{1}\right) x_{0}, y_{0}\right]
\end{aligned}
$$

ou seja, $\delta\left(x_{1}, y_{1}\right) x_{0}$ comuta com qualquer elemento de $\mathcal{A}_{0}$, o que implica

$$
\begin{aligned}
\delta\left(x_{1}, y_{1}\right) x_{0} y_{0} & =y_{0}\left(\delta\left(x_{1}, y_{1}\right) x_{0}\right) \\
& =y_{0} x_{0} \delta\left(x_{1}, y_{1}\right) \\
& =\delta\left(x_{1}, y_{1}\right)\left(y_{0} x_{0}\right)
\end{aligned}
$$


pois $\delta\left(x_{1}, y_{1}\right)$ também comuta com qualquer elemento de $\mathcal{A}_{0}$. Portanto, $\delta\left(x_{1}, y_{1}\right)\left[x_{0}, y_{0}\right]=0$.

Analogamente,

$$
\begin{aligned}
x_{0} y_{0} \delta\left(x_{1}, y_{1}\right) & =x_{0}\left(\delta\left(x_{1}, y_{1}\right) y_{0}\right) \\
& =\delta\left(x_{1}, y_{1}\right)\left(y_{0} x_{0}\right) \\
& =y_{0} x_{0} \delta\left(x_{1}, y_{1}\right)
\end{aligned}
$$

isto é, $\left[x_{0}, y_{0}\right] \delta\left(x_{1}, y_{1}\right)=0$. Substituindo $y_{0}$ por $y_{0} z_{0}$, onde $y_{0}$ e $z_{0}$ pertencem a $\mathcal{A}_{0}$, obtemos

$$
\begin{aligned}
0 & =\delta\left(x_{1}, y_{1}\right)\left[x_{0}, y_{0} z_{0}\right] \\
& =\delta\left(x_{1}, y_{1}\right)\left(x_{0} y_{0}\right) z_{0}-\delta\left(x_{1}, y_{1}\right) y_{0} z_{0} x_{0} \\
& =\delta\left(x_{1}, y_{1}\right) z_{0}\left(x_{0} y_{0}\right)-\delta\left(x_{1}, y_{1}\right) z_{0}\left(y_{0} x_{0}\right) \\
& =\delta\left(x_{1}, y_{1}\right) z_{0}\left[x_{0}, y_{0}\right] \mathrm{e} \\
0 & =\left[x_{0}, y_{0} z_{0}\right] \delta\left(x_{1}, y_{1}\right) \\
& =\left(x_{0} y_{0}\right) z_{0} \delta\left(x_{1}, y_{1}\right)-y_{0} z_{0} x_{0} \delta\left(x_{1}, y_{1}\right) \\
& =\left(x_{0} y_{0}\right) z_{0} \delta\left(x_{1}, y_{1}\right)-\left(y_{0} x_{0}\right) z_{0} \delta\left(x_{1}, y_{1}\right) \\
& =\left[x_{0}, y_{0}\right] z_{0} \delta\left(x_{1}, y_{1}\right),
\end{aligned}
$$

para qualquer $z_{0} \in \mathcal{A}_{0}$. Portanto, chegamos as seguintes igualdades

$$
\left[x_{0}, y_{0}\right] \mathcal{A}_{0} \delta\left(x_{1}, y_{1}\right)=0 \text { e } \delta\left(x_{1}, y_{1}\right) \mathcal{A}_{0}\left[x_{0}, y_{0}\right]=0
$$

para todos $x_{0}, y_{0} \in \mathcal{A}_{0}$ e $x_{1}, y_{1} \in \mathcal{A}_{1}$. Assim, como $\delta\left(x_{1}, y_{1}\right) \in \mathcal{A}_{1}$ e $\left[x_{0}, y_{0}\right] \in \mathcal{A}_{0}$, por (ii) do Lema 1.2 .5 , segue $\delta\left(x_{1}, y_{1}\right)=0$ ou $\left[x_{0}, y_{0}\right]=0$, para quaisquer $x_{0}, y_{0} \in \mathcal{A}_{0}$ e $x_{1}, y_{1} \in \mathcal{A}_{1}$. Entretanto, em vista de que sabemos $\left[\mathcal{A}_{0}, \mathcal{A}_{0}\right] \neq 0$, existem $x_{0}, y_{0} \in \mathcal{A}_{0}$ tais que $\left[x_{0}, y_{0}\right] \neq 0$. Logo, $\delta\left(x_{1}, y_{1}\right)=0$, para todos $x_{1}, y_{1} \in \mathcal{A}_{1}$.

Agora, por (iv) e (v) do Lema 2.2.3, temos

$$
\begin{gathered}
\delta\left(x_{0}, x_{1}\right) y_{1}=x_{0} \delta\left(x_{1}, y_{1}\right)-\delta\left(x_{0} x_{1}, y_{1}\right)=0 \mathrm{e} \\
y_{1} \delta\left(x_{0}, x_{1}\right)=\delta\left(x_{1}, y_{1}\right) x_{0}-\delta\left(x_{1} x_{0}, y_{1}\right)=0,
\end{gathered}
$$

para quaisquer $x_{0} \in \mathcal{A}_{0}$ e $x_{1}, y_{1} \in \mathcal{A}_{1}$. Aplicando (ii) do Lema 1.2.5, segue $\delta\left(\mathcal{A}_{0}, \mathcal{A}_{1}\right)=0$. Portanto, $\delta\left(\mathcal{A}_{1}, \mathcal{A}_{0}\right)=0$, por (i) do Lema 2.2.3. Então, estão provadas

$$
\delta\left(\mathcal{A}_{0}, \mathcal{A}_{0}\right)=\delta\left(\mathcal{A}_{0}, \mathcal{A}_{1}\right)=\delta\left(\mathcal{A}_{1}, \mathcal{A}_{0}\right)=\delta\left(\mathcal{A}_{1}, \mathcal{A}_{1}\right)=0
$$

isto é, $D$ é uma superderivação de grau 1 , o que contradiz a hipótese. Logo, $\mathcal{A}_{0}$ é uma álgebra comutativa.

Prosseguindo, suponha $D\left(\mathcal{A}_{0}\right)=0$. Assim, $D\left(x_{0} y_{0}\right)=0=D\left(x_{0}\right) y_{0}+x_{0} D\left(y_{0}\right)$, para todos $x_{0}, y_{0} \in \mathcal{A}_{0}$, e podemos aplicar todos os itens do Lema 2.2.3. Por (iii), temos $\left[\delta\left(\mathcal{A}_{1}, \mathcal{A}_{1}\right), \mathcal{A}_{0}\right]=0$, mas como $\delta\left(\mathcal{A}_{1}, \mathcal{A}_{1}\right) \subseteq \mathcal{A}_{1}$ e $\mathcal{A}_{0}$ é comutativa, segue de (ii) do Lema 1.2.6, que vale $\delta\left(\mathcal{A}_{1}, \mathcal{A}_{1}\right)=0$ ou $\mathcal{A}$ é comutativa. Se $\mathcal{A}$ é comutativa, e se $x_{0}, y_{0} \in \mathcal{A}_{0}$ e $x_{1}, y_{1} \in \mathcal{A}_{1}$, então, $x_{0} \circ_{s} y_{0}=x_{0} y_{0}$, $x_{0} \circ_{s} y_{1}=x_{0} y_{1}, x_{1} \circ_{s} y_{0}=x_{1} y_{0}$ e $x_{1} \circ_{s} y_{1}=0$, o que implica 
- $D\left(x_{0} y_{0}\right)=D\left(x_{0} \circ_{s} y_{0}\right)=D\left(x_{0}\right) \circ_{s} y_{0}+x_{0} \circ_{s} D\left(y_{0}\right)=D\left(x_{0}\right) y_{0}+x_{0} D\left(y_{0}\right)$;

- $D\left(x_{0} y_{1}\right)=D\left(x_{0} \circ_{s} y_{1}\right)=D\left(x_{0}\right) \circ_{s} y_{1}+x_{0} \circ_{s} D\left(y_{1}\right)=0+x_{0} D\left(y_{1}\right)=D\left(x_{0}\right) y_{1}+x_{0} D\left(y_{1}\right)$;

- $D\left(x_{1} y_{0}\right)=D\left(x_{1} \circ_{s} y_{0}\right)=D\left(x_{1}\right) \circ_{s} y_{0}-x_{1} \circ_{s} D\left(y_{0}\right)=D\left(x_{1}\right) y_{0}-0=D\left(x_{1}\right) y_{0}-x_{1} D\left(y_{0}\right)$;

- $D\left(x_{1} y_{1}\right)=0=D\left(x_{1} \circ_{s} y_{1}\right)=D\left(x_{1}\right) \circ_{s} y_{1}-x_{1} \circ_{s} D\left(y_{1}\right)=D\left(x_{1}\right) y_{1}-x_{1} D\left(y_{1}\right)$,

ou seja, $D$ é superderivação de grau 1 , o que contradiz a hipótese. Portanto, $\delta\left(\mathcal{A}_{1}, \mathcal{A}_{1}\right)=0$. Além disso, por (iv) do Lema 2.2.3, temos

$$
\delta\left(x_{0}, x_{1}\right) y_{1}=x_{0} \delta\left(x_{1}, y_{1}\right)-\delta\left(x_{0} x_{1}, y_{1}\right)=0
$$

e por $(\mathbf{v})$, vale

$$
y_{1} \delta\left(x_{0}, x_{1}\right)=\delta\left(x_{1}, y_{1}\right) x_{0}-\delta\left(x_{1} x_{0}, y_{1}\right)=0
$$

para qualquer $y_{1} \in \mathcal{A}_{1}$. Assim, substituindo $y_{1}$ por $y_{0} y_{1}$ e por $y_{1} y_{0}$, onde $y_{0} \in \mathcal{A}_{0}$ é um elemento qualquer, obtemos $\delta\left(x_{0}, x_{1}\right) \mathcal{A}_{0} y_{1}=y_{1} \mathcal{A}_{0} \delta\left(x_{0}, x_{1}\right)=0$, pois $\delta\left(x_{0}, x_{1}\right) \in \mathcal{A}_{0}$ e $y_{1} \in \mathcal{A}_{1}$. Aplicando (ii) do Lema 1.2.5, concluímos $\delta\left(x_{0}, x_{1}\right)=0$, para todos $x_{0} \in \mathcal{A}_{0}, x_{1} \in \mathcal{A}_{1}$, uma vez que podemos tomar $y_{1} \neq 0$. Desse modo, está provado $\delta\left(\mathcal{A}_{0}, \mathcal{A}_{1}\right)=0$, o que implica $\delta\left(\mathcal{A}_{1}, \mathcal{A}_{0}\right)=0$, por (i) do Lema 2.2.3. Porém, já demonstramos que vale $\delta\left(\mathcal{A}_{0}, \mathcal{A}_{0}\right)=0$, pois $\mathcal{A}_{0}$ é comutativa, e também $\delta\left(\mathcal{A}_{1}, \mathcal{A}_{1}\right)=0$. Desse modo, $D$ é uma superderivação de grau 1 , o que é uma contradição. Logo, $D\left(\mathcal{A}_{0}\right) \neq 0$.

Finalmente, somente falta provar que $\mathcal{A}$ é comutativa. Sejam $x_{0} \in \mathcal{A}_{0}$ tal que $D\left(x_{0}\right) \neq 0$ e $x_{1} \in \mathcal{A}_{1}$. Para qualquer $y_{0} \in \mathcal{A}_{0}$, temos

$$
x_{1}^{2}\left(D\left(x_{0}\right) y_{0} x_{1}\right)=D\left(x_{0}\right) y_{0} x_{1} x_{1}^{2}=D\left(x_{0}\right)\left(y_{0} x_{1}^{2}\right) x_{1}=D\left(x_{0}\right) x_{1}^{2} y_{0} x_{1},
$$

e para todo $y_{1} \in \mathcal{A}_{1}$, vale

$$
x_{1}^{2}\left(D\left(x_{0}\right) y_{1}\right) x_{1}=D\left(x_{0}\right) y_{1} x_{1}^{2} x_{1}=D\left(x_{0}\right)\left(y_{1} x_{1}\right) x_{1}^{2}=D\left(x_{0}\right) x_{1}^{2} y_{1} x_{1},
$$

pois $\mathcal{A}_{0}$ é comutativa. Da primeira igualdade, concluímos $\left[x_{1}^{2}, D\left(x_{0}\right)\right] y_{0} x_{1}=0$, para todo $y_{0} \in \mathcal{A}_{0}$, e da segunda resulta $\left[x_{1}^{2}, D\left(x_{0}\right)\right] y_{1} x_{1}=0$, para qualquer $y_{1} \in \mathcal{A}_{1}$. Portanto, $\left[x_{1}^{2}, D\left(x_{0}\right)\right] \mathcal{A} x_{1}=0$. Agora, pelo fato de $\mathcal{A}$ ser uma superálgebra prima, se $x_{1} \neq 0$, então, $\left[x_{1}^{2}, D\left(x_{0}\right)\right]=0$. Além disso, se $x_{1}=0$, então, obviamente $\left[x_{1}^{2}, D\left(x_{0}\right)\right]=0$. Desse modo, $x_{1}^{2} \in Z(\mathcal{A})$, para todo $x_{1} \in \mathcal{A}_{1}$, por (ii) do Lema 1.2.6, pois $x_{1}^{2} \in \mathcal{A}_{0}$ e $0 \neq D\left(x_{0}\right) \in \mathcal{A}_{1}$.

Agora, pelo Lema 2.2.2, segue

$$
\begin{aligned}
0=D(0)=D\left(\left[x_{1}^{2}, y\right]\right) & =\left[\left[D\left(x_{1}\right), x_{1}\right]_{s}, y\right]_{s}+\left[x_{1}^{2}, D(y)\right] \\
& =\left[\left[D\left(x_{1}\right), x_{1}\right], y\right]_{s}
\end{aligned}
$$

para todo $y \in \mathcal{A}$. Particularmente, a igualdade $\left[\left[D\left(x_{1}\right), x_{1}\right], \mathcal{A}_{0}\right]=0$ é válida. Portanto, se $0 \neq$ $\left[D\left(x_{1}\right), x_{1}\right] \in \mathcal{A}_{1}$, então, o item (ii) do Lema 1.2.6 implica que $\mathcal{A}$ é comutativa, como queremos provar. Suponhamos, então, $\left[D\left(x_{1}\right), x_{1}\right]=0$, para todo $x_{1} \in \mathcal{A}_{1}$. Afirmamos que vale $D\left(x_{1}\right) \in Z(\mathcal{A})$, para todo $x_{1} \in \mathcal{A}_{1}$. Com efeito, se $x_{1}=0$, então, $D\left(x_{1}\right)=0 \in Z(\mathcal{A})$. Caso contrário, $D\left(x_{1}\right) \in Z(\mathcal{A})$, por (ii) do Lema 1.2.6, pois $D\left(x_{1}\right) \in \mathcal{A}_{0}$. 
Pela definição de superderivação de Jordan de grau 1, obtemos

$$
\begin{aligned}
D\left(x_{1}^{2} \circ y_{1}\right) & =D\left(x_{1}^{2} \circ_{s} y_{1}\right) \\
& =D\left(x_{1}^{2}\right) \circ_{s} y_{1}+x_{1}^{2} \circ_{s} D\left(y_{1}\right) \\
& =\frac{1}{2}\left(D\left(x_{1}^{2}\right) y_{1}-y_{1} D\left(x_{1}^{2}\right)\right)+x_{1}^{2} \circ D\left(y_{1}\right) \\
& =\frac{1}{2}\left[D\left(x_{1}^{2}\right), y_{1}\right]+x_{1}^{2} \circ D\left(y_{1}\right),
\end{aligned}
$$

para quaisquer $x_{1}, y_{1} \in \mathcal{A}_{1}$. Assim, $D\left(x_{1}^{2} \circ y_{1}\right) \in Z(\mathcal{A})$ e $x_{1}^{2} \circ D\left(y_{1}\right) \in Z(\mathcal{A})$, pois $x_{1}^{2} \in Z(\mathcal{A})$ e $D\left(\mathcal{A}_{1}\right) \subseteq Z(\mathcal{A})$, o que implica $\left[D\left(x_{1}^{2}\right), y_{1}\right]=2 D\left(x_{1}^{2} \circ y_{1}\right)-2 x_{1}^{2} \circ D\left(y_{1}\right) \in Z(\mathcal{A})$.

Logo, $\left[\left[D\left(x_{1}^{2}\right), y_{1}\right], y_{1}\right]=0 \mathrm{e}$

$$
\begin{aligned}
0 & =-\left[\left[D\left(x_{1}^{2}\right), y_{1}\right], y_{1}\right] \\
& =-\left[D\left(x_{1}^{2}\right), y_{1}\right] y_{1}+y_{1}\left[D\left(x_{1}^{2}\right), y_{1}\right] \\
& =-D\left(x_{1}^{2}\right) y_{1}^{2}+y_{1} D\left(x_{1}^{2}\right) y_{1}+y_{1}\left[D\left(x_{1}^{2}\right), y_{1}\right] \\
& =-y_{1}^{2} D\left(x_{1}^{2}\right)+y_{1} D\left(x_{1}^{2}\right) y_{1}+y_{1}\left[D\left(x_{1}^{2}\right), y_{1}\right] \\
& =2 y_{1}\left[D\left(x_{1}^{2}\right), y_{1}\right]
\end{aligned}
$$

pois $y_{1}^{2} \in Z(\mathcal{A})$. Analogamente, também temos $\left[D\left(x_{1}^{2}\right), y_{1}\right] y_{1}=0$. Assim, substituindo $y_{1}$ por $y_{1} y_{0}$ e $y_{0} y_{1}$, onde $y_{0} \in \mathcal{A}_{0}$ é qualquer, obtemos

$$
y_{1} \mathcal{A}_{0}\left[D\left(x_{1}^{2}\right), y_{1}\right]=\left[D\left(x_{1}^{2}\right), y_{1}\right] \mathcal{A}_{0} y_{1}=0 .
$$

Escolhendo $y_{1} \neq 0$ e aplicando (ii) do Lema 1.2.5, concluímos $\left[D\left(x_{1}^{2}\right), y_{1}\right]=0$. Portanto, esta igualdade ocorre para todo $y_{1} \in \mathcal{A}_{1}$. Agora, por (iii) do Lema 1.2.6, temos $D\left(x_{1}^{2}\right)=0$, para todo $x_{1} \in \mathcal{A}_{1}$, ou $\mathcal{A}$ é comutativa. Suponhamos, então, que a primeira premissa é válida. A linearização da igualdade resulta em

$$
D\left(x_{1} y_{1}+y_{1} x_{1}\right)=0 \text {, para quaisquer } x_{1}, y_{1} \in \mathcal{A}_{1},
$$

ou seja, $D\left(x_{1} y_{1}\right)=-D\left(y_{1} x_{1}\right)$. Assim, como $D\left(\mathcal{A}_{1}\right) \subseteq Z(\mathcal{A})$, pela definição de superderivação de Jordan de grau 1 , temos

$$
\begin{aligned}
D\left(x_{1} \circ_{s} y_{1}\right) & =D\left(x_{1}\right) \circ_{s} y_{1}-x_{1} \circ_{s} D\left(y_{1}\right) \\
D\left(x_{1} y_{1}\right)-D\left(y_{1} x_{1}\right) & =D\left(x_{1}\right) y_{1}+y_{1} D\left(x_{1}\right)-x_{1} D\left(y_{1}\right)-D\left(y_{1}\right) x_{1} \\
2 D\left(x_{1} y_{1}\right) & =2\left(D\left(x_{1}\right) y_{1}-x_{1} D\left(y_{1}\right)\right),
\end{aligned}
$$

de onde concluímos que $\delta\left(x_{1}, y_{1}\right)=0$, para todos $x_{1}, y_{1} \in \mathcal{A}_{1}$, pois $\Phi$ tem característica diferente de 2 , isto é, $\delta\left(\mathcal{A}_{1}, \mathcal{A}_{1}\right)=0$. 
Agora, temos

$$
\begin{aligned}
\delta\left(x_{1} x_{0}, y_{1}\right)+\delta\left(x_{1}, x_{0}\right) y_{1}= & D\left(x_{1} x_{0} y_{1}\right)-D\left(x_{1} x_{0}\right) y_{1}+x_{1} x_{0} D\left(y_{1}\right)+D\left(x_{1} x_{0}\right) y_{1} \\
& -D\left(x_{1}\right) x_{0} y_{1}+x_{1} D\left(x_{0}\right) y_{1} \\
= & D\left(x_{1} x_{0} y_{1}\right)+x_{1} x_{0} D\left(y_{1}\right)-D\left(x_{1}\right) x_{0} y_{1}+x_{1} D\left(x_{0}\right) y_{1} \\
= & \delta\left(x_{1}, x_{0} y_{1}\right)-x_{1} D\left(x_{0} y_{1}\right)-x_{1} \delta\left(x_{0}, y_{1}\right)+x_{1} D\left(x_{0} y_{1}\right) \\
= & \delta\left(x_{1}, x_{0} y_{1}\right)-x_{1} \delta\left(x_{0}, y_{1}\right),
\end{aligned}
$$

para quaisquer $x_{0} \in \mathcal{A}_{0}, x_{1}, y_{1} \in \mathcal{A}_{1}$, o que resulta em

$$
\delta\left(x_{1}, x_{0}\right) y_{1}=x_{1} \delta\left(y_{1}, x_{0}\right),
$$

por (i) do Lema 2.2.3. Em particular, escolhendo $y_{1}=x_{1}$, obtemos $\delta\left(x_{1}, x_{0}\right) x_{1}=x_{1} \delta\left(x_{1}, x_{0}\right)$, o que implica $\left[\delta\left(x_{1}, x_{0}\right), x_{1}\right]=0$. Além disso se $0 \neq x_{1} \in \mathcal{A}_{1}$, então, $\delta\left(x_{1}, x_{0}\right) \in \mathcal{A}_{0}$ é um elemento do centro de $\mathcal{A}$, por (ii) do Lema 1.2.6; e trivialmente se $x_{1}=0$, então, $\delta\left(x_{1}, x_{0}\right)=0 \in Z(\mathcal{A})$. Desse modo, pela equação $(2.6)$, temos

$$
\begin{aligned}
\delta\left(x_{1}, x_{0}\right)\left[y_{1}, x_{1}\right] & =\delta\left(x_{1}, x_{0}\right) y_{1} x_{1}-\delta\left(x_{1}, x_{0}\right) x_{1} y_{1} \\
& =x_{1} \delta\left(y_{1}, x_{0}\right) x_{1}-x_{1} \delta\left(x_{1}, x_{0}\right) y_{1} \\
& =x_{1}^{2} \delta\left(y_{1}, x_{0}\right)-x_{1}^{2} \delta\left(x_{1}, x_{0}\right)=0 .
\end{aligned}
$$

Desse modo, $\delta\left(x_{1}, x_{0}\right) a\left[y_{1}, x_{1}\right]=a \delta\left(x_{1}, x_{0}\right)\left[y_{1}, x_{1}\right]=0$, para todo $a \in \mathcal{A}$. Assim, como $\mathcal{A}$ é uma superálgebra prima e $x_{0} \in \mathcal{A}_{0}$ e $y_{1} \in \mathcal{A}_{1}$ são elementos quaisquer, concluímos $\delta\left(x_{1}, \mathcal{A}_{0}\right)=0$ ou $\left[\mathcal{A}_{1}, x_{1}\right]=0$, o que implica $\delta\left(\mathcal{A}_{1}, \mathcal{A}_{0}\right)=0$ ou $\mathcal{A}$ é comutativa, por (iii) do Lema 1.2.6. Finalmente, se $\delta\left(\mathcal{A}_{1}, \mathcal{A}_{0}\right)=0$, então, a igualdade $\delta\left(\mathcal{A}_{0}, \mathcal{A}_{1}\right)=0$ também é válida. Neste caso, já provamos

$$
\delta\left(\mathcal{A}_{1}, \mathcal{A}_{1}\right)=\delta\left(\mathcal{A}_{1}, \mathcal{A}_{0}\right)=\delta\left(\mathcal{A}_{0}, \mathcal{A}_{1}\right)=0
$$

Porém, como por hipótese $D$ não é uma superderivação, necessariamente devemos ter

$$
\delta\left(\mathcal{A}_{0}, \mathcal{A}_{0}\right) \neq 0
$$

Entretanto, temos o seguinte

$$
\begin{aligned}
\delta\left(x_{0}, y_{0} x_{1}\right)+x_{0} \delta\left(y_{0}, x_{1}\right)= & D\left(x_{0} y_{0} x_{1}\right)-D\left(x_{0}\right) y_{0} x_{1}-x_{0} D\left(y_{0}\right) x_{1}-x_{0} y_{0} D\left(x_{1}\right) \\
= & D\left(x_{0} y_{0} x_{1}\right)-D\left(x_{0} y_{0}\right) x_{1}-x_{0} y_{0} D\left(x_{1}\right)+D\left(x_{0} y_{0}\right) x_{1} \\
& -D\left(x_{0}\right) y_{0} x_{1}-x_{0} D\left(y_{0}\right) x_{1} \\
= & \delta\left(x_{0} y_{0}, x_{1}\right)+\delta\left(x_{0}, y_{0}\right) x_{1} .
\end{aligned}
$$

o que implica $\delta\left(x_{0}, y_{0}\right) x_{1}=0$. Multiplicando esta igualdade à direita por $\delta\left(x_{0}, y_{0}\right)$, obtemos

$$
\delta\left(x_{0}, y_{0}\right) \mathcal{A}_{1} \delta\left(x_{0}, y_{0}\right)=0
$$

pois $x_{1} \in \mathcal{A}_{1}$ um elemento qualquer. Todavia, por (i) do Lema 1.2.5, temos $\delta\left(x_{0}, y_{0}\right)=0$, para 
todos $x_{0}, y_{0} \in \mathcal{A}_{0}$, contradizendo a hipótese. Portanto, em qualquer caso, $\mathcal{A}$ é comutativa.

Encerramos o capítulo apresentando um exemplo de superderivação de Jordan própria de grau 1 , ilustrando o teorema anterior.

Exemplo 2.2.3. Considere $\Phi[X]$ a álgebra dos polinômios sobre um corpo $\Phi$ e seja $\mathcal{A}=\Phi[X] \oplus$ $\Phi[X]$ a superálgebra com graduação dada por $\mathcal{A}_{0}=\Phi[X] \oplus 0$ e $\mathcal{A}_{1}=0 \oplus \Phi[X]$, e multiplicação definida como segue: $\left(p_{1}, q_{1}\right) \cdot\left(p_{2}, q_{2}\right)=\left(p_{1} p_{2}+q_{1} q_{2}, p_{1} q_{2}+q_{1} p_{2}\right)$. Definimos $D: \mathcal{A} \rightarrow \mathcal{A}$ por $D(p, q)=\left(0, p^{\prime}\right)$, onde $p^{\prime}$ é a derivada de $p$, para todo $(p, q) \in \mathcal{A}$. Então, $D$ é uma superderivação de Jordan própria de grau 1 de $\mathcal{A}$. Com efeito, escolhendo $(0, x),(x, 0) \in \mathcal{A}$, temos $D((0, x)(x, 0))=$ $D\left(0, x^{2}\right)=(0,0)$, mas $D(0, x)(x, 0)-(0, x) D(x, 0)=(0,0)(x, 0)-(0, x)(0,1)=(x, 0)$.

Corolário 2.2.1. Toda superderivação de Jordan em uma superálgebra associativa prima, cuja parte par é não comutativa, é uma superderivação.

Demonstração. É uma consequência dos Teoremas 2.2.1 e 2.2.2. 


\section{Capítulo 3}

\section{Derivações e superderivações de Jordan em anéis e superálgebras semiprimas}

Neste capítulo, apresentamos alguns resultados de Matej Brešar [7] e de Maja Fošner [11], os quais estendem o caso primo, que foi estudado no capítulo anterior, para o caso semiprimo.

\subsection{Derivações de Jordan em anéis semiprimos}

Inicialmente, recorde que um anel $A$ é semiprimo se não possui ideais nilpotentes não nulos. Isso equivale a satisfazer a propriedade: "se $a \in A$ é um elemento tal que $a A a=0$, então, $a=0$ ".

Nesta seção, o resultado principal que pretendemos provar afirma que toda derivação de Jordan de um anel semiprimo de característica diferente de 2 é uma derivação. A fim de demonstrá-lo, usaremos alguns fatos que já foram verificados no capítulo anterior. Iremos manter a notação $a^{b}=$ $D(a b)-D(a) b-a D(b)$, onde $a, b \in A$ e $D: A \rightarrow A$ é uma derivação de Jordan de um anel semiprimo $A$. Assim, já sabemos que são válidas as seguintes propriedades:

1. Para quaisquer $a, b \in A$, temos

$$
\begin{aligned}
a^{b}+b^{a} & =D(a b)-D(a) b-a D(b)+D(b a)-D(b) a-b D(a) \\
& =D(a b+b a)-D(a) b-b D(a)-a D(b)-D(b) a=0,
\end{aligned}
$$

pois $D$ é derivação de Jordan. Portanto, $a^{b}=-b^{a}$.

2. Para todos $a, b, c \in A$, seguem

$$
\begin{aligned}
a^{b+c} & =D(a(b+c))-D(a)(b+c)-a D(b+c) \\
& =D(a b)-D(a) b-a D(b)+D(a c)-D(a) c-a D(c) \\
& =a^{b}+a^{c} .
\end{aligned}
$$

Além disso, dados $a, b, x \in A$, pelo Teorema 2.1.1, vale

$$
a^{b} x[a, b]+[a, b] x a^{b}=0 .
$$


O próximo resultado, assim como a Proposição 2.2.2, estabelece condições para que uma derivação de Jordan seja uma derivação.

Teorema 3.1.1. (Brešar, [7]) Se $A$ é um anel semiprimo de característica diferente de 2 e $D: A \rightarrow A$ é uma derivação de Jordan de $A$, então, $D$ é uma derivação.

Demonstração. Na nossa notação, queremos mostrar que a igualdade $a^{b}=0$ é válida, para quaisquer $a, b \in A$. Pelos Teorema 2.1.1 e Lema 1.1.6 já sabemos que, para todos $a, b, x \in A$, temos

$$
a^{b} x[a, b]=0 .
$$

Substituindo $b$ por $b+c$ na igualdade acima, segue $a^{b+c} x[a, b+c]=0$, o que implica $a^{b} x[a, c]+$ $a^{c} x[a, b]=0$. Por essa relação e por (3.2), obtemos $\left(a^{b} x[a, c]\right) y\left(a^{b} x[a, c]\right)=-a^{b}\left(x[a, c] y a^{c} x\right)[a, b]=$ 0 , para quaisquer $a, b, c, x, y \in A$. Assim, como $A$ é um anel semiprimo, concluímos

$$
a^{b} x[a, c]=0
$$

para todos $a, b, c, x \in A$. Trocando $a$ por $a+d$ última igualdade, temos $(a+d)^{b} x[a+d, c]=0$, o que implica $a^{b} x[d, c]+d^{b} x[a, c]=0$. Por esta igualdade e por (3.3), obtemos $\left(a^{b} x[d, c]\right) y\left(a^{b} x[d, c]\right)=$ $-a^{b}\left(x[d, c] y d^{b} x\right)[a, c]=0$. Desse modo, segue

$$
a^{b} x[d, c]=0
$$

para todos $a, b, c, d, x \in A$, pois $A$ é um anel semiprimo. Em particular, $\left[a^{b}, c\right] x\left[a^{b}, c\right]=a^{b}(c x)\left[a^{b}, c\right]-$ $c a^{b} x\left[a^{b}, c\right]=0$, para quaisquer $a, b, c, x \in A$, o que nos leva a $\left[a^{b}, c\right]=0$, para todos $a, b, c \in A$, visto que $A$ é um anel semiprimo. Portanto, $a^{b} \in Z(A)$, para quaisquer $a, b \in A$. Agora, usando (3.4) obtemos $\left(a^{b}[d, c]\right) x\left(a^{b}[d, c]\right)=a^{b}\left([d, c] x a^{b}\right)[d, c]=0$, de onde segue

$$
a^{b}[d, c]=0
$$

para quaisquer $a, b, c, d \in A$. Usando novamente as propriedades da notação, temos

$$
\begin{aligned}
2\left(a^{b}\right)^{2} & =a^{b} a^{b}+a^{b}\left(-b^{a}\right) \\
& =a^{b}\left(a^{b}-b^{a}\right) \\
& =a^{b}(D(a b)-D(a) b-a D(b)-D(b a)+D(b) a+b D(a)) \\
& =a^{b}(D(a b-b a)+[b, D(a)]+[D(b), a]) .
\end{aligned}
$$

Além disso, de (3.5), decorre $a^{b}[b, D(a)]=a^{b}[D(b), a]=0$, o que reduz a relação acima a

$$
2\left(a^{b}\right)^{2}=a^{b} D([a, b]) .
$$

Novamente por (3.5), vale $a^{b}[a, b]=0$, o que nos permite concluir $a^{b}[a, b]+[a, b] a^{b}=0$, pois $a^{b} \in Z(A)$. Assim, como $D$ é uma derivação de Jordan, temos as seguintes igualdades

$$
0=D(0)=D\left(a^{b}[a, b]+[a, b] a^{b}\right)=D\left(a^{b}\right)[a, b]+a^{b} D([a, b])+D([a, b]) a^{b}+[a, b] D\left(a^{b}\right),
$$


que podem ser reescritas como

$$
D\left(a^{b}\right)[a, b]+4\left(a^{b}\right)^{2}+[a, b] D\left(a^{b}\right)=0 .
$$

pela igualdade (3.6) e pois $a^{b} \in Z(A)$.

Finalmente, multiplicando (3.7) por $a^{b}$, podemos concluir que vale $4\left(a^{b}\right)^{3}=0$, para todos $a, b \in A$, por (3.5), o que implica $\left(a^{b}\right)^{3}=0$, pois a característica de $A$ é diferente de 2 . Desse modo, $a^{b}$ é um elemento nilpotente que pertence a $Z(A)$. Portanto, pelo Lema 1.1.5, temos $a^{b}=0$, para todos $a, b \in A$. Logo, $D$ é uma derivação de $A$.

\subsection{Superderivações de Jordan em superálgebras semiprimas}

Conforme as definições dadas anteriormente, uma superálgebra $\mathcal{A}$ é semiprima se não possui ideais graduados nilpotentes não nulos, equivalentemente a superálgebra $\mathcal{A}$ deve satisfazer "se $a$ é um elemento homogêneo arbitrário de $\mathcal{A}$ tal que $a \mathcal{A} a=0$, então, $a=0$ ".

O objetivo dessa seção é generalizar o resultado anterior para superálgebras. Para isso, usaremos alguns fatos já enunciados e provados no capítulo anterior, como por exemplo, o Lema 1.2.4 que mostra que se $\mathcal{A}=\mathcal{A}_{0} \oplus \mathcal{A}_{1}$ é uma superálgebra associativa semiprima, então, $\mathcal{A}$ e $\mathcal{A}_{0}$ são álgebras associativas semiprimas.

Ao longo de toda essa seção, $\mathcal{A}=\mathcal{A}_{0} \oplus \mathcal{A}_{1}$ denotará uma superálgebra, isto é, $\mathcal{A}$ é uma álgebra $\mathbb{Z}_{2}$-graduada sobre um anel $\Phi$ comutativo, com identidade e tal que $\frac{1}{2} \in \Phi$, associativa semiprima e $D=D_{0}+D_{1}$ uma superderivação de Jordan de $\mathcal{A}$, onde $D_{i}$ representa uma superderivação de Jordan de grau $i$ de $\mathcal{A}$, para $i=0,1$. Além disso, temos as operações $\circ$ e $\circ_{s}$ dadas por

$$
\begin{gathered}
a \circ b=\frac{1}{2}(a b+b a), \text { para todos } a, b \in \mathcal{A} \text { e } \\
a \circ_{s} b=\frac{1}{2}\left(a b+(-1)^{|a||b|} b a\right), \text { para quaisquer } a, b \in \mathcal{A}_{0} \cup \mathcal{A}_{1} .
\end{gathered}
$$

Definimos também aplicações bilineares $\delta_{i}: \mathcal{A} \times \mathcal{A} \rightarrow \mathcal{A}$, onde $i=0,1$, por

$$
\delta_{i}(x, y)=D_{i}(x y)-D_{i}(x) y-(-1)^{i|x|} x D_{i}(y),
$$

para todos $x, y \in \mathcal{A}_{0} \cup \mathcal{A}_{1}$. Observe que apesar de termos provado anteriormente que $\delta_{i}$ é biaditiva, a bilinearidade segue trivialmente, pois $D_{i}$ é $\Phi$-linear. Além disso, claramente $D_{i}$ é uma superderivação de grau $i$ de $\mathcal{A}$ se, e somente se, $\delta_{i}=0$, onde $i=0,1$.

Se $x$ e $y$ são elementos de $\mathcal{A}$ tais que $x \mathcal{A} y=0$, o que vamos denotar por $x \perp y$, então, seguem $x y=y x=y \mathcal{A} x=0$. Com efeito, $($ yax $) \mathcal{A}($ yax $)=y a(x \mathcal{A} y) a x=0$, para todo $a \in \mathcal{A}$, o que implica $y \mathcal{A} x=0$, pois $\mathcal{A}$ também é semiprima como álgebra; e $(x y) \mathcal{A}(x y)=x(y \mathcal{A} x) y=0$ e $(y x) \mathcal{A}(y x)=y(x \mathcal{A} y) x=0$ implicam $x y=y x=0$.

A fim de provarmos a generalização desejada, necessitaremos de alguns lemas auxiliares.

Lema 3.2.1. Se $x$ e $y$ são elementos homogêneos de $\mathcal{A}$ tais que $x \perp y$, então, $x \circ_{s} D_{i}(y)=0$, onde $i=0,1$.

Demonstração. Por hipótese, $x \mathcal{A} y=0$, o que implica $y \mathcal{A} x=x y=y x=0$. Desse modo, $x \circ_{s} y=$ 
$x y+(-1)^{|x||y|} y x=0$. Assim, temos $D_{i}\left(x \circ_{s} y\right)=0$, pois $D_{i}$ é $\Phi$-linear, o que implica $D_{i}(x) \circ_{s} y+$ $(-1)^{i|x|} x \circ_{s} D_{i}(y)=0$, uma vez que $D_{i}$ é uma superderivação de Jordan de $\mathcal{A}$ de grau $i$.

Consequentemente, as igualdades são válidas

$$
\begin{aligned}
0 & =(x a)\left(D_{i}(x) \circ_{s} y+(-1)^{i|x|} x \circ_{s} D_{i}(y)\right) \\
& =x\left(a D_{i}(x)\right) y+(-1)^{\left|D_{i}(x)\right||y|}(x a y) D_{i}(x)+(-1)^{i|x|}(x a)\left(x \circ_{s} D_{i}(y)\right) \\
& =(-1)^{i|x|}(x a)\left(x \circ_{s} D_{i}(y)\right),
\end{aligned}
$$

para todo $a \in \mathcal{A}$, ou seja, $x a\left(x \circ_{s} D_{i}(y)\right)=0$, o que resulta em

$$
\left(x \circ_{s} D_{i}(y)\right) a\left(x \circ_{s} D_{i}(y)\right)=x\left(D_{i}(y) a\right)\left(x \circ_{s} D_{i}(y)\right)+(-1)^{|x|\left|D_{i}(y)\right|} D_{i}(y)\left(x a\left(x \circ_{s} D_{i}(y)\right)\right)=0,
$$

para todo $a \in \mathcal{A}$, isto é, $\left(x \circ_{s} D_{i}(y)\right) \mathcal{A}\left(x \circ_{s} D_{i}(y)\right)=0$, de onde concluímos $x \circ_{s} D_{i}(y)=0$, pois $\mathcal{A}$ é semiprima.

Lema 3.2.2. São válidas as seguintes igualdades:

(i) $\delta_{i}\left(x_{0}, y_{0}\right)=-\delta_{i}\left(y_{0}, x_{0}\right), \delta_{i}\left(x_{0}, y_{1}\right)=-\delta_{i}\left(y_{1}, x_{0}\right)$ e $\delta_{i}\left(x_{1}, y_{1}\right)=\delta_{i}\left(y_{1}, x_{1}\right)$, para quaisquer $x_{0}, y_{0} \in$ $\mathcal{A}_{0}$ e $x_{1}, y_{1} \in \mathcal{A}_{1}$

(ii) $D_{i}\left(\left[x_{1}^{2}, y\right]\right)=\left[\left[D_{i}\left(x_{1}\right), x_{1}\right]_{s}, y\right]_{s}+\left[x_{1}^{2}, D_{i}(y)\right]$, para todos $x_{1} \in \mathcal{A}_{1}$ e $y \in \mathcal{A}$.

Demonstração. As igualdades $\delta_{i}\left(x_{0}, y_{1}\right)=-\delta_{i}\left(y_{1}, x_{0}\right)$ e $\delta_{i}\left(x_{1}, y_{1}\right)=\delta_{i}\left(y_{1}, x_{1}\right)$ já foram demonstradas em (i) e (ii) do Lema 2.2.3 do Capítulo 2. Além disso, (ii) está provado no Lema 2.2 .2 do Capítulo 2. Finalmente, somente falta mostrar que a igualdade $\delta_{i}\left(x_{0}, y_{0}\right)=-\delta_{i}\left(y_{0}, x_{0}\right)$ é válida. Com efeito,

$$
\begin{aligned}
\delta_{i}\left(x_{0}, y_{0}\right)+\delta_{i}\left(y_{0}, x_{0}\right)= & D_{i}\left(x_{0} y_{0}\right)-D_{i}\left(x_{0}\right) y_{0}-(-1)^{i\left|x_{0}\right|} x_{0} D_{i}\left(y_{0}\right) \\
& +D_{i}\left(y_{0} x_{0}\right)-D_{i}\left(y_{0}\right) x_{0}-(-1)^{i\left|y_{0}\right|} y_{0} D_{i}\left(x_{0}\right) \\
= & D_{i}\left(x_{0} y_{0}+y_{0} x_{0}\right)-\left(D_{i}\left(x_{0}\right) y_{0}+y_{0} D_{i}\left(x_{0}\right)\right) \\
& -\left(x_{0} D_{i}\left(y_{0}\right)+D_{i}\left(y_{0}\right) x_{0}\right) \\
= & 2\left(D_{i}\left(x_{0} \circ_{s} y_{0}\right)-D_{i}\left(x_{0}\right) \circ_{s} y_{0}-(-1)^{i\left|x_{0}\right|} x_{0} \circ_{s} D_{i}\left(y_{0}\right)\right)=0,
\end{aligned}
$$

pois $D_{i}$ é superderivação de Jordan de grau $i$ de $\mathcal{A}$. Portanto, $\delta_{i}\left(x_{0}, y_{0}\right)=-\delta_{i}\left(y_{0}, x_{0}\right)$, para todos $x_{0}, y_{0} \in \mathcal{A}_{0}$.

Lema 3.2.3. Seja $U$ um ideal graduado de $\mathcal{A}$. Se $\delta_{i}\left(U_{0}, \mathcal{A}_{0}\right)=0$, então, as seguintes afirmações são verdadeiras:

(i) $\left[\delta_{i}\left(\mathcal{A}_{1}, \mathcal{A}_{1}\right), U_{0}\right]=0$;

(ii) $\delta_{i}\left(u_{0}, x_{1}\right) y_{1}=u_{0} \delta_{i}\left(x_{1}, y_{1}\right)-\delta_{i}\left(u_{0} x_{1}, y_{1}\right)$, para todos $u_{0} \in U_{0}$ e $x_{1}, y_{1} \in \mathcal{A}_{1}$;

(iii) $\delta_{i}\left(x_{0}, u_{1}\right) y_{1}=x_{0} \delta_{i}\left(u_{1}, y_{1}\right)-\delta_{i}\left(x_{0} u_{1}, y_{1}\right)$, para quaisquer $x_{0} \in \mathcal{A}_{0}, u_{1} \in U_{1}$ e $y_{1} \in \mathcal{A}_{1}$;

(iv) $(-1)^{i} y_{1} \delta_{i}\left(u_{0}, x_{1}\right)=\delta_{i}\left(x_{1} u_{0}, y_{1}\right)-\delta_{i}\left(x_{1}, y_{1}\right) u_{0}$, para todos $u_{0} \in U_{0}$ e $x_{1}, y_{1} \in \mathcal{A}_{1}$;

(v) $(-1)^{i} y_{1} \delta_{i}\left(x_{0}, u_{1}\right)=\delta_{i}\left(u_{1} x_{0}, y_{1}\right)-\delta_{i}\left(u_{1}, y_{1}\right) x_{0}$, para quaisquer $x_{0} \in \mathcal{A}_{0}, u_{1} \in U_{1}$ e $y_{1} \in \mathcal{A}_{1}$; 
(vi) $\delta_{0}\left(U_{0}, \mathcal{A}_{1}\right) \perp\left[\mathcal{A}_{0}, U_{0}\right]$;

(vii) $\delta_{1}\left(U_{0}, \mathcal{A}_{1}\right) \mathcal{A}_{1}\left[\mathcal{A}_{0}, U_{0}\right]=\left[\mathcal{A}_{0}, U_{0}\right] \mathcal{A}_{1} \delta_{1}\left(U_{0}, \mathcal{A}_{1}\right)=0$.

Demonstração. (i) Para cada $x_{1} \in \mathcal{A}_{1}$ e $u_{0} \in U_{0}$, temos

$$
\begin{aligned}
D_{i}\left(\left[x_{1}^{2}, u_{0}\right]\right)= & D_{i}\left(x_{1}^{2} u_{0}\right)-D_{i}\left(u_{0} x_{1}^{2}\right) \\
= & \delta_{i}\left(x_{1}^{2}, u_{0}\right)+D_{i}\left(x_{1}^{2}\right) u_{0}+(-1)^{i\left|x_{1}^{2}\right|} x_{1}^{2} D_{i}\left(u_{0}\right) \\
& -\delta_{i}\left(u_{0}, x_{1}^{2}\right)-D_{i}\left(u_{0}\right) x_{1}^{2}-(-1)^{i\left|u_{0}\right|} u_{0} D_{i}\left(x_{1}^{2}\right) \\
= & -2 \delta_{i}\left(u_{0}, x_{1}^{2}\right)+D_{i}\left(x_{1}^{2}\right) u_{0}+x_{1}^{2} D_{i}\left(u_{0}\right)-D_{i}\left(u_{0}\right) x_{1}^{2}-u_{0} D_{i}\left(x_{1}^{2}\right) \\
= & {\left[D_{i}\left(x_{1}^{2}\right), u_{0}\right]+\left[x_{1}^{2}, D_{i}\left(u_{0}\right)\right] }
\end{aligned}
$$

pois $x_{1}^{2} \in \mathcal{A}_{0} \delta_{i}\left(U_{0}, \mathcal{A}_{0}\right)=0$. Por outro lado, por (ii) do Lema 3.2.2, também vale

$$
D_{i}\left(\left[x_{1}^{2}, u_{0}\right]\right)=\left[\left[D_{i}\left(x_{1}\right), x_{1}\right]_{s}, u_{0}\right]_{s}+\left[x_{1}^{2}, D_{i}\left(u_{0}\right)\right] .
$$

Desse modo, igualando as duas expressões obtidas, segue $\left[\left[D_{i}\left(x_{1}\right), x_{1}\right]_{s}, u_{0}\right]_{s}=\left[D_{i}\left(x_{1}^{2}\right), u_{0}\right]$, o que equivale a $\left[D_{i}\left(x_{1}\right) x_{1}-(-1)^{\left|D_{i}\left(x_{1}\right)\right|\left|x_{1}\right|} x_{1} D_{i}\left(x_{1}\right), u_{0}\right]=\left[D_{i}\left(x_{1}^{2}\right), u_{0}\right]$. Portanto,

$$
\left[D_{i}\left(x_{1}^{2}\right)-D_{i}\left(x_{1}\right) x_{1}-(-1)^{i\left|x_{1}\right|} x_{1} D_{i}\left(x_{1}\right), u_{0}\right]=0
$$

isto é, $\left[\delta_{i}\left(x_{1}, x_{1}\right), u_{0}\right]=0$. Linearizando esta última igualdade, obtemos $\left[\delta_{i}\left(x_{1}, y_{1}\right), u_{0}\right]+$ $\left[\delta_{i}\left(y_{1}, x_{1}\right), u_{0}\right]=0$, o que implica $2\left[\delta_{i}\left(x_{1}, y_{1}\right), u_{0}\right]=0$, por (i) do Lema 3.2.2. Consequentemente, $\left[\delta_{i}\left(x_{1}, y_{1}\right), u_{0}\right]=0$, para todos $x_{1}, y_{1} \in \mathcal{A}_{1}, u_{0} \in U_{0}$, ou seja, $\left[\delta_{i}\left(\mathcal{A}_{1}, \mathcal{A}_{1}\right), U_{0}\right]=0$.

(ii) Dados $u_{0} \in U_{0}, x_{1}, y_{1} \in \mathcal{A}_{1}$, vamos determinar $D_{i}\left(u_{0} x_{1} y_{1}\right)$. Por um lado, temos

$$
\begin{aligned}
D_{i}\left(u_{0}\left(x_{1} y_{1}\right)\right) & =\delta_{i}\left(u_{0}, x_{1} y_{1}\right)+D_{i}\left(u_{0}\right) x_{1} y_{1}+(-1)^{i\left|u_{0}\right|} u_{0} D_{i}\left(x_{1} y_{1}\right) \\
& =D_{i}\left(u_{0}\right) x_{1} y_{1}+u_{0}\left(\delta_{i}\left(x_{1}, y_{1}\right)+D_{i}\left(x_{1}\right) y_{1}+(-1)^{i\left|x_{1}\right|} x_{1} D_{i}\left(y_{1}\right)\right) .
\end{aligned}
$$

Por outro lado, também vale

$$
\begin{aligned}
D_{i}\left(\left(u_{0} x_{1}\right) y_{1}\right)= & \delta_{i}\left(u_{0} x_{1}, y_{1}\right)+D_{i}\left(u_{0} x_{1}\right) y_{1}+(-1)^{i\left|u_{0} x_{1}\right|} u_{0} x_{1} D_{i}\left(y_{1}\right) \\
= & \delta_{i}\left(u_{0} x_{1}, y_{1}\right)+\left(\delta_{i}\left(u_{0}, x_{1}\right)+D_{i}\left(u_{0}\right) x_{1}+(-1)^{i\left|u_{0}\right|} u_{0} D_{i}\left(x_{1}\right)\right) y_{1} \\
& +(-1)^{i} u_{0} x_{1} D_{i}\left(y_{1}\right) .
\end{aligned}
$$

Igualando as duas expressões, obtemos $u_{0} \delta_{i}\left(x_{1}, y_{1}\right)=\delta_{i}\left(u_{0} x_{1}, y_{1}\right)+\delta_{i}\left(u_{0}, x_{1}\right) y_{1}$, ou seja,

$$
\delta_{i}\left(u_{0}, x_{1}\right) y_{1}=u_{0} \delta_{i}\left(x_{1}, y_{1}\right)-\delta_{i}\left(u_{0} x_{1}, y_{1}\right)
$$

(iii) Para cada $x_{0} \in \mathcal{A}_{0}, u_{1} \in U_{1}$ e $y_{1} \in \mathcal{A}_{1}$, calculemos $D_{i}\left(x_{0} u_{1} y_{1}\right)$. Por um lado, vale

$$
\begin{aligned}
D_{i}\left(x_{0}\left(u_{1} y_{1}\right)\right) & =\delta_{i}\left(x_{0}, u_{1} y_{1}\right)+D_{i}\left(x_{0}\right) u_{1} y_{1}+(-1)^{i\left|x_{0}\right|} x_{0} D_{i}\left(u_{1} y_{1}\right) \\
& =D_{i}\left(x_{0}\right) u_{1} y_{1}+x_{0}\left(\delta_{i}\left(u_{1}, y_{1}\right)+D_{i}\left(u_{1}\right) y_{1}+(-1)^{i\left|u_{1}\right|} u_{1} D_{i}\left(y_{1}\right)\right) .
\end{aligned}
$$


Por outro lado, também temos

$$
\begin{aligned}
D_{i}\left(\left(x_{0} u_{1}\right) y_{1}\right)= & \delta_{i}\left(x_{0} u_{1}, y_{1}\right)+D_{i}\left(x_{0} u_{1}\right) y_{1}+(-1)^{i\left|x_{0} u_{1}\right|} x_{0} u_{1} D_{i}\left(y_{1}\right) \\
= & \delta_{i}\left(x_{0} u_{1}, y_{1}\right)+\left(\delta_{i}\left(x_{0}, u_{1}\right)+D_{i}\left(x_{0}\right) u_{1}+(-1)^{i\left|x_{0}\right|} x_{0} D_{i}\left(u_{1}\right)\right) y_{1} \\
& +(-1)^{i} x_{0} u_{1} D_{i}\left(y_{1}\right) .
\end{aligned}
$$

A comparação das duas expressões obtidas, resulta em

$$
x_{0} \delta_{i}\left(u_{1}, y_{1}\right)=\delta_{i}\left(x_{0} u_{1}, y_{1}\right)+\delta_{i}\left(x_{0}, u_{1}\right) y_{1},
$$

ou seja, $\delta_{i}\left(x_{0}, u_{1}\right) y_{1}=x_{0} \delta_{i}\left(u_{1}, y_{1}\right)-\delta_{i}\left(x_{0} u_{1}, y_{1}\right)$.

(iv) Dados $u_{0} \in U_{0}, x_{1}, y_{1} \in \mathcal{A}_{1}$, vamos determinar $D_{i}\left(y_{1} x_{1} u_{0}\right)$. Por um lado, as seguintes igualdades são válidas

$$
\begin{aligned}
D_{i}\left(y_{1}\left(x_{1} u_{0}\right)\right)= & \delta_{i}\left(y_{1}, x_{1} u_{0}\right)+D_{i}\left(y_{1}\right) x_{1} u_{0}+(-1)^{i\left|y_{1}\right|} y_{1} D_{i}\left(x_{1} u_{0}\right) \\
= & \delta_{i}\left(y_{1}, x_{1} u_{0}\right)+D_{i}\left(y_{1}\right) x_{1} u_{0}+(-1)^{i} y_{1}\left(\delta_{i}\left(x_{1}, u_{0}\right)+D_{i}\left(x_{1}\right) u_{0}\right. \\
& \left.+(-1)^{i\left|x_{1}\right|} x_{1} D_{i}\left(u_{0}\right)\right) .
\end{aligned}
$$

Por outro lado, também sabemos

$$
\begin{aligned}
D_{i}\left(\left(y_{1} x_{1}\right) u_{0}\right) & =\delta_{i}\left(y_{1} x_{1}, u_{0}\right)+D_{i}\left(y_{1} x_{1}\right) u_{0}+(-1)^{i\left|y_{1} x_{1}\right|} y_{1} x_{1} D_{i}\left(u_{0}\right) \\
& =\left(\delta_{i}\left(y_{1}, x_{1}\right)+D_{i}\left(y_{1}\right) x_{1}+(-1)^{i\left|y_{1}\right|} y_{1} D_{i}\left(x_{1}\right)\right) u_{0}+y_{1} x_{1} D_{i}\left(u_{0}\right) .
\end{aligned}
$$

Igualando estas duas expressões, obtemos $\delta_{i}\left(y_{1}, x_{1} u_{0}\right)+(-1)^{i} y_{1} \delta_{i}\left(x_{1}, u_{0}\right)=\delta_{i}\left(y_{1}, x_{1}\right) u_{0}$, ou seja,

$$
(-1)^{i} y_{1} \delta_{i}\left(x_{1}, u_{0}\right)=\delta_{i}\left(x_{1} u_{0}, y_{1}\right)-\delta_{i}\left(x_{1}, y_{1}\right) u_{0}
$$

(v) Para cada $x_{0} \in \mathcal{A}_{0}, u_{1} \in U_{1}$ e $y_{1} \in \mathcal{A}_{1}$, calculemos $D_{i}\left(y_{1} u_{1} x_{0}\right)$. Por um lado, note

$$
\begin{aligned}
D_{i}\left(y_{1}\left(u_{1} x_{0}\right)\right)= & \delta_{i}\left(y_{1}, u_{1} x_{0}\right)+D_{i}\left(y_{1}\right) u_{1} x_{0}+(-1)^{i\left|y_{1}\right|} y_{1} D_{i}\left(u_{1} x_{0}\right) \\
= & \delta_{i}\left(y_{1}, u_{1} x_{0}\right)+D_{i}\left(y_{1}\right) u_{1} x_{0}+(-1)^{i} y_{1}\left(\delta_{i}\left(u_{1}, x_{0}\right)+D_{i}\left(u_{1}\right) x_{0}\right. \\
& \left.+(-1)^{i\left|u_{1}\right|} u_{1} D_{i}\left(x_{0}\right)\right),
\end{aligned}
$$

Por outro lado, também temos

$$
\begin{aligned}
D_{i}\left(\left(y_{1} u_{1}\right) x_{0}\right) & =\delta_{i}\left(y_{1} u_{1}, x_{0}\right)+D_{i}\left(y_{1} u_{1}\right) x_{0}+(-1)^{i\left|y_{1} u_{1}\right|} y_{1} u_{1} D_{i}\left(x_{0}\right) \\
& =\left(\delta_{i}\left(y_{1}, u_{1}\right)+D_{i}\left(y_{1}\right) u_{1}+(-1)^{i\left|y_{1}\right|} y_{1} D_{i}\left(u_{1}\right)\right) x_{0}+y_{1} u_{1} D_{i}\left(x_{0}\right) .
\end{aligned}
$$

A comparação das duas expressões obtidas, resulta em $\delta_{i}\left(y_{1}, u_{1} x_{0}\right)+(-1)^{i} y_{1} \delta_{i}\left(u_{1}, x_{0}\right)=$ $\delta_{i}\left(y_{1}, u_{1}\right) x_{0}$, ou seja,

$$
(-1)^{i} y_{1} \delta_{i}\left(x_{0}, u_{1}\right)=\delta_{i}\left(u_{1} x_{0}, y_{1}\right)-\delta_{i}\left(u_{1}, y_{1}\right) x_{0}
$$


(vi) Observe que pelos itens (i) e (ii), temos

$$
\left[\delta_{i}\left(u_{0}, x_{1}\right) y_{1}, u_{0}\right]=\left[u_{0} \delta_{i}\left(x_{1}, y_{1}\right), u_{0}\right]-\left[\delta_{i}\left(u_{0} x_{1}, y_{1}\right), u_{0}\right]=0
$$

para quaisquer $u_{0} \in U_{0}$ e $x_{1}, y_{1} \in \mathcal{A}_{1}$, isto é, $\left[\delta_{i}\left(u_{0}, \mathcal{A}_{1}\right) \mathcal{A}_{1}, u_{0}\right]=0$, para todo $u_{0} \in U_{0}$. Desse modo, as seguintes igualdades são válidas:

$$
\begin{aligned}
\delta_{i}\left(u_{0}, x_{1}\right) z_{1}\left[z_{0}, u_{0}\right]= & \delta_{i}\left(u_{0}, x_{1}\right) z_{1} z_{0} u_{0}-\delta_{i}\left(u_{0}, x_{1}\right) z_{1} u_{0} z_{0} \\
= & \delta_{i}\left(u_{0}, x_{1}\right) z_{1} z_{0} u_{0}-u_{0} \delta_{i}\left(u_{0}, x_{1}\right) z_{1} z_{0}+u_{0} \delta_{i}\left(u_{0}, x_{1}\right) z_{1} z_{0} \\
& -\delta_{i}\left(u_{0}, x_{1}\right) z_{1} u_{0} z_{0} \\
= & {\left[\delta_{i}\left(u_{0}, x_{1}\right)\left(z_{1} z_{0}\right), u_{0}\right]-\left[\delta_{i}\left(u_{0}, x_{1}\right) z_{1}, u_{0}\right] z_{0}=0 }
\end{aligned}
$$

para todos $u_{0} \in U_{0}, z_{0} \in \mathcal{A}_{0}$ e $x_{1}, z_{1} \in \mathcal{A}_{1}$, isto é,

$$
\delta_{i}\left(u_{0}, \mathcal{A}_{1}\right) \mathcal{A}_{1}\left[\mathcal{A}_{0}, u_{0}\right]=0
$$

para qualquer $u_{0} \in U_{0}$. Além disso, por (i) e (iv), também temos

$$
\begin{aligned}
{\left[y_{1} \delta_{i}\left(u_{0}, x_{1}\right), u_{0}\right] } & =(-1)^{i}\left[\delta_{i}\left(x_{1} u_{0}, y_{1}\right), u_{0}\right]-(-1)^{i}\left[\delta_{i}\left(x_{1}, y_{1}\right) u_{0}, u_{0}\right] \\
& =(-1)^{i}\left[\delta_{i}\left(x_{1} u_{0}, y_{1}\right), u_{0}\right]-(-1)^{i}\left[\delta_{i}\left(x_{1}, y_{1}\right), u_{0}\right] u_{0}=0
\end{aligned}
$$

para todos $x_{1}, y_{1} \in \mathcal{A}_{1}$. Substituindo $y_{1}$ por $z_{0} z_{1}$, onde $z_{i} \in \mathcal{A}_{i}, i=0,1$, obtemos

$$
\begin{aligned}
0 & =\left[\left(z_{0} z_{1}\right) \delta_{i}\left(u_{0}, x_{1}\right), u_{0}\right] \\
& =z_{0} z_{1} \delta_{i}\left(u_{0}, x_{1}\right) u_{0}-u_{0} z_{0} z_{1} \delta_{i}\left(u_{0}, x_{1}\right) \\
& =z_{0} u_{0} z_{1} \delta_{i}\left(u_{0}, x_{1}\right)-u_{0} z_{0} z_{1} \delta_{i}\left(u_{0}, x_{1}\right)+z_{0} z_{1} \delta_{i}\left(u_{0}, x_{1}\right) u_{0}-z_{0} u_{0} z_{1} \delta_{i}\left(u_{0}, x_{1}\right) \\
& =\left[z_{0}, u_{0}\right] z_{1} \delta_{i}\left(u_{0}, x_{1}\right)+z_{0}\left[z_{1} \delta_{i}\left(u_{0}, x_{1}\right), u_{0}\right] \\
& =\left[z_{0}, u_{0}\right] z_{1} \delta_{i}\left(u_{0}, x_{1}\right),
\end{aligned}
$$

isto é, $\left[\mathcal{A}_{0}, u_{0}\right] \mathcal{A}_{1} \delta_{i}\left(u_{0}, \mathcal{A}_{1}\right)=0$. Agora, a linearização de (3.9) resulta em

$$
\delta_{i}\left(u_{0}, x_{1}\right) y_{1}\left[z_{0}, v_{0}\right]+\delta_{i}\left(v_{0}, x_{1}\right) y_{1}\left[z_{0}, u_{0}\right]=0
$$

ou seja, $\delta_{i}\left(u_{0}, x_{1}\right) y_{1}\left[z_{0}, v_{0}\right]=-\delta_{i}\left(v_{0}, x_{1}\right) y_{1}\left[z_{0}, u_{0}\right]$, para quaisquer $u_{0}, v_{0} \in U_{0}, z_{0} \in \mathcal{A}_{0} \mathrm{e}$ $x_{1}, y_{1} \in \mathcal{A}_{1}$. Por esta última igualdade, temos

$$
\begin{aligned}
& \left(\delta_{i}\left(u_{0}, x_{1}\right) y_{1}\left[z_{0}, v_{0}\right]\right) a_{1}\left(\delta_{i}\left(u_{0}, x_{1}\right) y_{1}\left[z_{0}, v_{0}\right]\right) \\
& \quad=-\delta_{i}\left(u_{0}, x_{1}\right) y_{1}\left(\left[z_{0}, v_{0}\right] a_{1} \delta_{i}\left(v_{0}, x_{1}\right)\right) y_{1}\left[z_{0}, u_{0}\right]=0 \mathrm{e} \\
& \quad\left(\left[z_{0}, v_{0}\right] y_{1} \delta_{i}\left(u_{0}, x_{1}\right)\right) a_{1}\left(\left[z_{0}, v_{0}\right] y_{1} \delta_{i}\left(u_{0}, x_{1}\right)\right) \\
& \quad=-\left(\left[z_{0}, v_{0}\right] y_{1} \delta_{i}\left(v_{0}, x_{1}\right)\right) a_{1}\left[z_{0}, u_{0}\right] y_{1} \delta_{i}\left(u_{0}, x_{1}\right)=0,
\end{aligned}
$$

para todo $a_{1} \in \mathcal{A}_{1}$. Se $i=0$, então, $\delta_{0}\left(u_{0}, x_{1}\right) \in \mathcal{A}_{1}$ e $\left[z_{0}, u_{0}\right] \in \mathcal{A}_{0}$. Assim, por (ii) do Lema 1.2.8, para o caso particular $U=\mathcal{A}$, como $\delta_{0}\left(u_{0}, x_{1}\right) \mathcal{A}_{1}\left[z_{0}, u_{0}\right]=\left[z_{0}, u_{0}\right] \mathcal{A}_{1} \delta_{0}\left(u_{0}, x_{1}\right)=0$, concluímos $\delta_{0}\left(u_{0}, x_{1}\right) \mathcal{A}\left[z_{0}, u_{0}\right]=\left[z_{0}, u_{0}\right] \mathcal{A} \delta_{0}\left(u_{0}, x_{1}\right)=0$, para todo $u_{0} \in U_{0}$, de onde obtemos $\delta_{0}\left(u_{0}, x_{1}\right) a\left[z_{0}, v_{0}\right]+\delta_{0}\left(v_{0}, x_{1}\right) a\left[z_{0}, u_{0}\right]=0$, ou seja, $\delta_{0}\left(u_{0}, x_{1}\right) a\left[z_{0}, v_{0}\right]=-\delta_{0}\left(v_{0}, x_{1}\right) a\left[z_{0}, u_{0}\right]$, 
para todo $a \in \mathcal{A}$. Consequentemente, segue que são válidas as igualdades

$$
\begin{aligned}
& \left(\delta_{0}\left(u_{0}, x_{1}\right) y\left[z_{0}, v_{0}\right]\right) a\left(\delta_{0}\left(u_{0}, x_{1}\right) y\left[z_{0}, v_{0}\right]\right) \\
& \quad=-\delta_{0}\left(u_{0}, x_{1}\right) y\left(\left[z_{0}, v_{0}\right] a \delta_{0}\left(v_{0}, x_{1}\right)\right) y\left[z_{0}, u_{0}\right]=0
\end{aligned}
$$

para todo $a \in \mathcal{A}$. Portanto, $\delta_{0}\left(u_{0}, x_{1}\right) y\left[z_{0}, v_{0}\right]=0$, para todo $y \in \mathcal{A}$, pois $\mathcal{A}$ é semiprima. Logo, $\delta_{0}\left(U_{0}, \mathcal{A}_{1}\right) \mathcal{A}\left[\mathcal{A}_{0}, U_{0}\right]=0$, como queríamos provar.

(vii) Se $i=1$, então, $\left[z_{0}, v_{0}\right] \in \mathcal{A}_{0}$ e $\delta_{1}\left(u_{0}, x_{1}\right) \in \mathcal{A}_{0}$, para todos $u_{0}, v_{0} \in U_{0}, z_{0} \in \mathcal{A}_{0}$ e $x_{1} \in \mathcal{A}_{1}$. Desse modo, $\left[z_{0}, v_{0}\right] y_{1} \delta_{1}\left(u_{0}, x_{1}\right), \delta_{1}\left(u_{0}, x_{1}\right) y_{1}\left[z_{0}, v_{0}\right] \in \mathcal{A}_{1}$, para todo $y_{1} \in \mathcal{A}_{1}$. Agora, pelas igualdades (3.10) e (3.11), ocorrem

$$
\begin{gathered}
\left(\left[z_{0}, v_{0}\right] y_{1} \delta_{1}\left(u_{0}, x_{1}\right)\right) \mathcal{A}_{1}\left(\left[z_{0}, v_{0}\right] y_{1} \delta_{1}\left(u_{0}, x_{1}\right)\right)=0 \mathrm{e} \\
\left(\delta_{1}\left(u_{0}, x_{1}\right) y_{1}\left[z_{0}, v_{0}\right]\right) \mathcal{A}_{1}\left(\delta_{1}\left(u_{0}, x_{1}\right) y_{1}\left[z_{0}, v_{0}\right]\right)=0 .
\end{gathered}
$$

Portanto, (i) do Lema 1.2 .8 implica $\left[z_{0}, v_{0}\right] y_{1} \delta_{1}\left(u_{0}, x_{1}\right)=\delta_{1}\left(u_{0}, x_{1}\right) y_{1}\left[z_{0}, v_{0}\right]=0$, para todo $y_{1} \in \mathcal{A}_{1}$, isto é,

$$
\left[z_{0}, v_{0}\right] \mathcal{A}_{1} \delta_{1}\left(u_{0}, x_{1}\right)=\delta_{1}\left(u_{0}, x_{1}\right) \mathcal{A}_{1}\left[z_{0}, v_{0}\right]=0
$$

\subsubsection{Superderivações de Jordan de grau 0}

Nesta seção, vamos estudar as superderivações de Jordan de grau 0. A seguinte notação é adotada: $\left[\mathcal{A}_{0}, \mathcal{A}_{0}\right]$ representa o subgrupo aditivo de $\mathcal{A}$ gerado pelos elementos da forma $\left[x_{0}, y_{0}\right]=$ $x_{0} y_{0}-y_{0} x_{0}$, com $x_{0}, y_{0} \in \mathcal{A}_{0}$, e definimos $U$ como sendo o ideal de $\mathcal{A}$ gerado por $\left[\mathcal{A}_{0}, \mathcal{A}_{0}\right]$. Observe que $U$ é um ideal graduado de $\mathcal{A}$.

Teorema 3.2.1. (Fošner, [11]) Para a aplicação bilinear $\delta_{0}$, definida em (3.8), e o ideal $U$ de $\mathcal{A}$, definido anteriormente, vale $\delta_{0}(U, \mathcal{A})=0$.

Demonstração. Primeiramente, observe que como $\left.D_{0}\right|_{\mathcal{A}_{0}}$ é uma derivação de Jordan e $\mathcal{A}_{0}$ é uma álgebra semiprima, já vimos na Seção 3.1 que ocorre

$$
\delta_{0}\left(\mathcal{A}_{0}, \mathcal{A}_{0}\right)=0
$$

isto é, $\left.D_{0}\right|_{\mathcal{A}_{0}}$ é uma derivação em $\mathcal{A}_{0}$. Assim, aplicando (vi) do Lema 3.2 .3 para o caso $U=\mathcal{A}$, obtemos

$$
\delta_{0}\left(\mathcal{A}_{0}, \mathcal{A}_{1}\right) \perp\left[\mathcal{A}_{0}, \mathcal{A}_{0}\right]
$$

Em particular, para quaisquer $x_{0}, y_{0} \in \mathcal{A}_{0}, y_{0}, y_{1} \in \mathcal{A}_{1}$ e $z \in \mathcal{A}$ valem as seguintes igualdades

$$
\begin{aligned}
& {\left[y_{1} \delta_{0}\left(x_{0}, x_{1}\right), y_{0}\right] z\left[y_{1} \delta_{0}\left(x_{0}, x_{1}\right), y_{0}\right]} \\
& \quad=y_{1}\left(\delta_{0}\left(x_{0}, x_{1}\right)\left(y_{0} z\right)\left[y_{1} \delta_{0}\left(x_{0}, x_{1}\right), y_{0}\right]\right)-y_{0} y_{1}\left(\delta_{0}\left(x_{0}, x_{1}\right) z\left[y_{1} \delta_{0}\left(x_{0}, x_{1}\right), y_{0}\right]\right)=0 \quad \mathrm{e} \\
& \quad\left[\delta_{0}\left(x_{0}, x_{1}\right) y_{1}, y_{0}\right] z\left[\delta_{0}\left(x_{0}, x_{1}\right) y_{1}, y_{0}\right] \\
& \quad=\left(\delta_{0}\left(x_{0}, x_{1}\right)\left(y_{1} y_{0} z\right)\left[\delta_{0}\left(x_{0}, x_{1}\right) y_{1}, y_{0}\right]\right)-y_{0}\left(\delta_{0}\left(x_{0}, x_{1}\right)\left(y_{1} z\right)\left[\delta_{0}\left(x_{0}, x_{1}\right) y_{1}, y_{0}\right]\right)=0,
\end{aligned}
$$

pois $y_{1} \delta_{0}\left(x_{0}, x_{1}\right), \delta_{0}\left(x_{0}, x_{1}\right) y_{1} \in \mathcal{A}_{0}$. Desse modo, como $\mathcal{A}$ é uma superálgebra semiprima, concluímos

$$
\left[y_{1} \delta_{0}\left(x_{0}, x_{1}\right), y_{0}\right]=\left[\delta_{0}\left(x_{0}, x_{1}\right) y_{1}, y_{0}\right]=0
$$


para todo $y_{0} \in \mathcal{A}_{0}$, isto é,

$$
\mathcal{A}_{1} \delta_{0}\left(\mathcal{A}_{0}, \mathcal{A}_{1}\right), \delta_{0}\left(\mathcal{A}_{0}, \mathcal{A}_{1}\right) \mathcal{A}_{1} \in Z\left(\mathcal{A}_{0}\right)
$$

o que implica $\delta_{0}\left(x_{0}, x_{1}\right)^{2} \in Z(\mathcal{A})$, para todos $x_{0} \in \mathcal{A}_{0}$ e $x_{1} \in \mathcal{A}_{1}$, pelo Lema 1.2.7.

Prosseguindo, dado $u_{0} \in U_{0} \subseteq \mathcal{A}_{0}$, observemos que vale $\delta_{0}\left(u_{0}, x_{1}\right) y_{1} \perp v$, para todos $v \in U_{0} \cup U_{1}$ e $x_{1}, y_{1} \in \mathcal{A}_{1}$, pois $\delta_{0}\left(\mathcal{A}_{0}, \mathcal{A}_{1}\right) \perp\left[\mathcal{A}_{0}, \mathcal{A}_{0}\right]$ e $U$ é o ideal gerado pelos elementos da forma $\left[x_{0}, y_{0}\right]$, onde $x_{0}, y_{0} \in \mathcal{A}_{0}$. Agora, aplicando o Lema 3.2.1, temos $\delta_{0}\left(u_{0}, x_{1}\right) y_{1} \circ_{s} D_{0}(v)=0$, ou seja,

$$
\delta_{0}\left(u_{0}, x_{1}\right) y_{1} D_{0}(v)+D_{0}(v) \delta_{0}\left(u_{0}, x_{1}\right) y_{1}=0
$$

Assim, para $y_{1}=\delta_{0}\left(u_{0}, x_{1}\right) \in \mathcal{A}_{1}$, a igualdade se torna $\delta_{0}\left(u_{0}, x_{1}\right)^{2} D_{0}(v)+D_{0}(v) \delta_{0}\left(u_{0}, x_{1}\right)^{2}=0$, o que implica $2 \delta_{0}\left(u_{0}, x_{1}\right)^{2} D_{0}(v)=0$, pois $\delta_{0}\left(u_{0}, x_{1}\right)^{2} \in Z(\mathcal{A})$. Portanto, $\delta_{0}\left(u_{0}, x_{1}\right)^{2} D_{0}(v)=0$. Além disso, pela definição da aplicação $\delta_{0}$, também temos

$$
\left(\delta_{0}\left(u_{0}, x_{1}\right) y_{1}\right)^{2}=\delta_{0}\left(u_{0}, x_{1}\right) y_{1}\left(D_{0}\left(u_{0} x_{1}\right)-D_{0}\left(u_{0}\right) x_{1}-u_{0} D_{0}\left(x_{1}\right)\right) y_{1},
$$

Desse modo, novamente escolhendo $y_{1}=\delta_{0}\left(u_{0}, x_{1}\right)$, chegamos a

$$
\begin{aligned}
\delta_{0}\left(u_{0}, x_{1}\right)^{4}= & \left(\delta_{0}\left(u_{0}, x_{1}\right)^{2} D_{0}\left(u_{0} x_{1}\right)-\left(\delta_{0}\left(u_{0}, x_{1}\right)^{2} D_{0}\left(u_{0}\right)\right) x_{1}\right. \\
& \left.-\left(\delta_{0}\left(u_{0}, x_{1}\right)^{2} u_{0}\right) D_{0}\left(x_{1}\right)\right) \delta_{0}\left(u_{0}, x_{1}\right)^{2}=0
\end{aligned}
$$

pois $u_{0}, u_{0} x_{1} \in U_{0} \cup U_{1}$. Consequentemente, também vale

$$
\delta_{0}\left(u_{0}, x_{1}\right)^{2} a \delta_{0}\left(u_{0}, x_{1}\right)^{2}=\delta_{0}\left(u_{0}, x_{1}\right)^{4} a=0,
$$

para todo $a \in \mathcal{A}$, o que implica $\delta_{0}\left(u_{0}, x_{1}\right)^{2}=0$, pois $\mathcal{A}$ é semiprima.

Note que se $v \in U_{1}$, multiplicando (3.15) por $\delta_{0}\left(u_{0}, x_{1}\right) y_{1}$ à esquerda, obtemos

$$
\begin{aligned}
0 & =\left(\delta_{0}\left(u_{0}, x_{1}\right) y_{1}\right)^{2} D_{0}(v)+\delta_{0}\left(u_{0}, x_{1}\right) y_{1} D_{0}(v) \delta_{0}\left(u_{0}, x_{1}\right) y_{1} \\
& =\left(\delta_{0}\left(u_{0}, x_{1}\right) y_{1}\right)^{2} D_{0}(v)+D_{0}(v) \delta_{0}\left(u_{0}, x_{1}\right)^{2} y_{1}^{2} \quad\left(\delta_{0}\left(\mathcal{A}_{0}, \mathcal{A}_{1}\right) \mathcal{A}_{1} \subseteq Z\left(\mathcal{A}_{0}\right)\right) \\
& =\left(\delta_{0}\left(u_{0}, x_{1}\right) y_{1}\right)^{2} D_{0}(v) .
\end{aligned}
$$

Se $v \in U_{0}$, então, por (3.14) e (3.15), segue

$$
0=\delta_{0}\left(u_{0}, x_{1}\right) y_{1} D_{0}(v)+D_{0}(v) \delta_{0}\left(u_{0}, x_{1}\right) y_{1}=2 \delta_{0}\left(u_{0}, x_{1}\right) y_{1} D_{0}(v)
$$

pois $D_{0}(v) \in \mathcal{A}_{0}$, o que implica $\delta_{0}\left(u_{0}, x_{1}\right) y_{1} D_{0}(v)=0$.

Agora, multiplicando (3.16) por $\delta_{0}\left(u_{0}, x_{1}\right) y_{1}$, temos

$$
\begin{aligned}
\left(\delta_{0}\left(u_{0}, x_{1}\right) y_{1}\right)^{3}= & \left(\delta_{0}\left(u_{0}, x_{1}\right) y_{1}\right)^{2} D_{0}\left(u_{0} x_{1}\right)-\left(\delta_{0}\left(u_{0}, x_{1}\right) y_{1}\right)^{2} D_{0}\left(u_{0}\right) x_{1} \\
& \left.-\left(\delta_{0}\left(u_{0}, x_{1}\right) y_{1}\right)^{2} u_{0} D_{0}\left(x_{1}\right)\right) y_{1}=0
\end{aligned}
$$

pois $u_{0} \in U_{0}$ e $u_{0} x_{1} \in U_{1}$. Portanto $\delta_{0}\left(u_{0}, x_{1}\right) y_{1}$ é um elemento nilpotente de $Z\left(\mathcal{A}_{0}\right)$, o que implica $\delta_{0}\left(u_{0}, x_{1}\right) y_{1}=0$, pelo Lema 1.1 .5 , pois $\mathcal{A}_{0}$ é uma álgebra semiprima. Consequentemente, $\delta_{0}\left(u_{0}, x_{1}\right) y_{1} \delta_{0}\left(u_{0}, x_{1}\right)=0$, para todo $y_{1} \in \mathcal{A}_{1}$. Desse modo, aplicando o Lema 1.2 .8 para o caso $U=\mathcal{A}$, obtemos $\delta_{0}\left(u_{0}, x_{1}\right)=0$, pois este último é um elemento de $\mathcal{A}_{1}$, para todos $u_{0} \in U_{0}$ e $x_{1} \in \mathcal{A}_{1}$. Assim, está provado $\delta_{0}\left(U_{0}, \mathcal{A}_{1}\right)=0$. 
Além disso, dado $u_{1} \in U_{1}$, temos $\delta_{0}\left(u_{1}, x_{0}\right) y_{1} \perp v$, para todos $v \in U_{0} \cup U_{1}, x_{0} \in \mathcal{A}_{0}$ e $y_{1} \in \mathcal{A}_{1}$, pois $\delta_{0}\left(u_{1}, x_{0}\right)=-\delta_{0}\left(x_{0}, u_{1}\right), \delta_{0}\left(\mathcal{A}_{0}, \mathcal{A}_{1}\right) \perp\left[\mathcal{A}_{0}, \mathcal{A}_{0}\right]$ e $U$ é o ideal gerado pelos elementos da forma $\left[z_{0}, w_{0}\right]$, onde $\left.z_{0}, w_{0} \in \mathcal{A}_{0}\right)$. Aplicando o Lema 3.2.1, obtemos $\delta_{0}\left(u_{1}, x_{0}\right) y_{1} \circ_{s} D_{0}(v)=0$, ou seja,

$$
\delta_{0}\left(u_{1}, x_{0}\right) y_{1} D_{0}(v)+D_{0}(v) \delta_{0}\left(u_{1}, x_{0}\right) y_{1}=0 .
$$

Em particular, tomando $y_{1}=\delta_{0}\left(u_{1}, x_{0}\right) \in \mathcal{A}_{1}$, segue $\delta_{0}\left(u_{1}, x_{0}\right)^{2} D_{0}(v)+D_{0}(v) \delta_{0}\left(u_{1}, x_{0}\right)^{2}=0$, o que implica $2 \delta_{0}\left(u_{1}, x_{0}\right)^{2} D_{0}(v)=0$, pois $\delta_{0}\left(u_{1}, x_{0}\right)^{2} \in Z(\mathcal{A})$. Portanto, $\delta_{0}\left(u_{1}, x_{0}\right)^{2} D_{0}(v)=0$.

Agora, pela definição da aplicação $\delta_{0}$, também temos

$$
\left(\delta_{0}\left(u_{1}, x_{0}\right) y_{1}\right)^{2}=\delta_{0}\left(u_{1}, x_{0}\right) y_{1}\left(D_{0}\left(u_{1} x_{0}\right)-D_{0}\left(u_{1}\right) x_{0}-u_{1} D_{0}\left(x_{0}\right)\right) y_{1} .
$$

Analogamente, escolhendo $y_{1}=\delta_{0}\left(u_{1}, x_{0}\right)$, chegamos a

$$
\begin{aligned}
\delta_{0}\left(u_{1}, x_{0}\right)^{4}= & \left(\delta_{0}\left(u_{1}, x_{0}\right)^{2} D_{0}\left(u_{1} x_{0}\right)-\left(\delta_{0}\left(u_{1}, x_{0}\right)^{2} D_{0}\left(u_{1}\right)\right) x_{0}\right. \\
& \left.-\left(\delta_{0}\left(u_{1}, x_{0}\right)^{2} u_{1}\right) D_{0}\left(x_{0}\right)\right) \delta_{0}\left(u_{1}, x_{0}\right)^{2}=0,
\end{aligned}
$$

pois $u_{1}$ e $u_{1} x_{0}$ pertencem a $U_{0} \cup U_{1}$. Consequentemente,

$$
\delta_{0}\left(u_{1}, x_{0}\right)^{2} a \delta_{0}\left(u_{1}, x_{0}\right)^{2}=\delta_{0}\left(u_{1}, x_{0}\right)^{4} a=0
$$

para todo $a \in \mathcal{A}$, o que implica $\delta_{0}\left(u_{1}, x_{0}\right)^{2}=0$, pela semiprimalidade de $\mathcal{A}$.

Prosseguindo, dado $v \in U_{1}$, multiplicando (3.17) à esquerda por $\delta_{0}\left(u_{1}, x_{0}\right) y_{1}$, obtemos

$$
\begin{aligned}
0 & =\left(\delta_{0}\left(u_{1}, x_{0}\right) y_{1}\right)^{2} D_{0}(v)+\delta_{0}\left(u_{1}, x_{0}\right) y_{1} D_{0}(v) \delta_{0}\left(u_{1}, x_{0}\right) y_{1} \\
& =\left(\delta_{0}\left(u_{1}, x_{0}\right) y_{1}\right)^{2} D_{0}(v)+D_{0}(v) \delta_{0}\left(u_{1}, x_{0}\right)^{2} y_{1}^{2} \quad\left(\delta_{0}\left(\mathcal{A}_{1}, \mathcal{A}_{0}\right) \mathcal{A}_{1} \subseteq Z\left(\mathcal{A}_{0}\right)\right) \\
& =\left(\delta_{0}\left(u_{1}, x_{0}\right) y_{1}\right)^{2} D_{0}(v) .
\end{aligned}
$$

Repetindo o mesmo processo para (3.18), segue

$$
\begin{aligned}
\left(\delta_{0}\left(u_{1}, x_{0}\right) y_{1}\right)^{3}= & \left(\delta_{0}\left(u_{1}, x_{0}\right) y_{1}\right)^{2} D_{0}\left(u_{1} x_{0}\right)-\left(\delta_{0}\left(u_{1}, x_{0}\right) y_{1}\right)^{2} D_{0}\left(u_{1}\right) x_{0} \\
& \left.-\left(\delta_{0}\left(u_{1}, x_{0}\right) y_{1}\right)^{2} u_{1} D_{0}\left(x_{0}\right)\right) y_{1}=0
\end{aligned}
$$

pois $u_{1}, u_{1} x_{0} \in U_{1}$, isto é, $\delta_{0}\left(u_{1}, x_{0}\right) y_{1}$ é um elemento nilpotente que pertence ao centro de $\mathcal{A}_{0}$. Assim, como $\mathcal{A}_{0}$ é uma álgebra semiprima, concluímos $\delta_{0}\left(u_{1}, x_{0}\right) y_{1}=0$, pelo Lema 1.1.5. Desse modo, claramente $\delta_{0}\left(u_{1}, x_{0}\right) y_{1} \delta_{0}\left(u_{1}, x_{0}\right)=0$, para todo $y_{1} \in \mathcal{A}_{1}$. Agora, aplicando o Lema 1.2 .8 para o caso $U=\mathcal{A}$, obtemos $\delta_{0}\left(u_{1}, x_{0}\right)=0$, pois este último é um elemento de $\mathcal{A}_{1}$, para todos $u_{1} \in U_{1}, x_{0} \in \mathcal{A}_{0}$, o que prova que a igualdade $\delta_{0}\left(U_{1}, \mathcal{A}_{0}\right)=0$ é válida.

Dados $u_{1} \in U_{1}$ e $x_{1} \in \mathcal{A}_{1}$, como $x_{1}^{2} \in \mathcal{A}_{0}$, temos $\delta_{0}\left(x_{1}^{2}, u_{1}\right)=0$, o que implica

$$
\begin{aligned}
D_{0}\left(\left[x_{1}^{2}, u_{1}\right]\right) & =D_{0}\left(x_{1}^{2} u_{1}\right)-D_{0}\left(u_{1} x_{1}^{2}\right) \\
& =\delta_{0}\left(x_{1}^{2}, u_{1}\right)+D_{0}\left(x_{1}^{2}\right) u_{1}+x_{1}^{2} D_{0}\left(u_{1}\right)-\delta_{0}\left(u_{1}, x_{1}^{2}\right)-D_{0}\left(u_{1}\right) x_{1}^{2}-u_{1} D_{0}\left(x_{1}^{2}\right) \\
& =\left[D_{0}\left(x_{1}^{2}\right), u_{1}\right]+\left[x_{1}^{2}, D_{0}\left(u_{1}\right)\right] .
\end{aligned}
$$


Por outro lado, (ii) do Lema 3.2.2 nos fornece

$$
\begin{aligned}
D_{0}\left(\left[x_{1}^{2}, u_{1}\right]\right) & =\left[\left[D_{0}\left(x_{1}\right), x_{1}\right]_{s}, u_{1}\right]_{s}+\left[x_{1}^{2}, D_{0}\left(u_{1}\right)\right] \\
& =\left[D_{0}\left(x_{1}\right) x_{1}+x_{1} D_{0}\left(x_{1}\right), u_{1}\right]+\left[x_{1}^{2}, D_{0}\left(u_{1}\right)\right] .
\end{aligned}
$$

Assim, comparando as igualdades encontradas, obtemos

$$
\left[D_{0}\left(x_{1}^{2}\right), u_{1}\right]=\left[D_{0}\left(x_{1}\right) x_{1}+x_{1} D_{0}\left(x_{1}\right), u_{1}\right]
$$

ou seja, $\left[\delta_{0}\left(x_{1}, x_{1}\right), u_{1}\right]=0$, para todos $u_{1} \in U_{1}, x_{1} \in \mathcal{A}_{1}$.

Além disso, como $\delta_{0}\left(\mathcal{A}_{0}, \mathcal{A}_{0}\right)=0$, temos $\delta_{0}\left(U_{0}, \mathcal{A}_{0}\right)=0$, o que implica $\left[\delta_{0}\left(\mathcal{A}_{1}, \mathcal{A}_{1}\right), U_{0}\right]=0$, por (i) do Lema 3.2.3. Desse modo, vale $\left[\delta_{0}\left(x_{1}, x_{1}\right), U_{0}\right]=0$, e consequentemente, $\left[\delta_{0}\left(x_{1}, x_{1}\right), U\right]=0$, para todo $x_{1} \in \mathcal{A}_{1}$. Logo, linearizando esta igualdade, obtemos $\left[\delta_{0}\left(x_{1}, y_{1}\right), u\right]+\left[\delta_{0}\left(y_{1}, x_{1}\right), u\right]=0$, para todo $u \in U$, o que equivale a $\left[\delta_{0}\left(x_{1}, y_{1}\right), U\right]=0$, pelo Lema 3.2 .2 e pois $\frac{1}{2} \in \Phi$, isto é,

$$
\left[\delta_{0}\left(\mathcal{A}_{1}, \mathcal{A}_{1}\right), U\right]=0
$$

Prosseguindo, de (3.14) e de (i) e (ii) do Lema 3.2.3, no caso $U=\mathcal{A}$, decorrem as seguintes igualdades

$$
\begin{aligned}
{\left[x_{0} \delta_{0}\left(x_{1}, y_{1}\right), y_{0}\right]=} & x_{0} \delta_{0}\left(x_{1}, y_{1}\right) y_{0}-y_{0} x_{0} \delta_{0}\left(x_{1}, y_{1}\right) \\
= & \delta_{0}\left(x_{0}, x_{1}\right) y_{1} y_{0}+\delta_{0}\left(x_{0} x_{1}, y_{1}\right) y_{0}-y_{0} \delta_{0}\left(x_{0}, x_{1}\right) y_{1} \\
& -y_{0} \delta_{0}\left(x_{0} x_{1}, y_{1}\right) \\
= & {\left[\delta_{0}\left(x_{0}, x_{1}\right) y_{1}, y_{0}\right]+\left[\delta_{0}\left(x_{0} x_{1}, y_{1}\right), y_{0}\right]=0, }
\end{aligned}
$$

para quaisquer $x_{0}, y_{0} \in \mathcal{A}_{0}, x_{1}, y_{1} \in \mathcal{A}_{1}$, o que implica

$$
\begin{aligned}
0 & =\left[x_{0} \delta_{0}\left(x_{1}, y_{1}\right), y_{0}\right] \\
& =x_{0} \delta_{0}\left(x_{1}, y_{1}\right) y_{0}-y_{0} x_{0} \delta_{0}\left(x_{1}, y_{1}\right) \\
& =x_{0} \delta_{0}\left(x_{1}, y_{1}\right) y_{0}-x_{0} y_{0} \delta_{0}\left(x_{1}, y_{1}\right)+x_{0} y_{0} \delta_{0}\left(x_{1}, y_{1}\right)-y_{0} x_{0} \delta_{0}\left(x_{1}, y_{1}\right) \\
& =x_{0}\left[\delta_{0}\left(x_{1}, y_{1}\right), y_{0}\right]+\left[x_{0}, y_{0}\right] \delta_{0}\left(x_{1}, y_{1}\right) \\
& =\left[x_{0}, y_{0}\right] \delta_{0}\left(x_{1}, y_{1}\right),
\end{aligned}
$$

para todos $x_{0}, y_{0} \in \mathcal{A}_{0}, x_{1}, y_{1} \in \mathcal{A}_{1}$, isto é,

$$
\left[\mathcal{A}_{0}, \mathcal{A}_{0}\right] \delta_{0}\left(\mathcal{A}_{1}, \mathcal{A}_{1}\right)=0
$$

Agora, temos $a_{0}\left[x_{0}, y_{0}\right], a_{1}\left[x_{0}, y_{0}\right] \in U$, para cada $a_{0}, x_{0}, y_{0} \in \mathcal{A}_{0}$ e $a_{1}, x_{1}, y_{1} \in \mathcal{A}_{1}$, pois $U=$ ideal $\left\langle\left[\mathcal{A}_{0}, \mathcal{A}_{0}\right]\right\rangle$; e $\delta_{0}\left(x_{1}, y_{1}\right) a_{i}\left[x_{0}, y_{0}\right]=a_{i}\left[x_{0}, y_{0}\right] \delta_{0}\left(x_{1}, y_{1}\right)=0$, para $i=0,1$, por $(3.19)$ e $(3.20)$, ou seja, $\delta_{0}\left(\mathcal{A}_{1}, \mathcal{A}_{1}\right) \mathcal{A}_{i}\left[\mathcal{A}_{0}, \mathcal{A}_{0}\right]=0$. Portanto, temos

$$
\delta_{0}\left(\mathcal{A}_{1}, \mathcal{A}_{1}\right) \perp\left[\mathcal{A}_{0}, \mathcal{A}_{0}\right] .
$$

Além disso, dado $u_{1} \in U_{1}$, note que, por (3.21), vale $\delta_{0}\left(u_{1}, x_{1}\right) \perp v$, para todos $x_{1} \in \mathcal{A}_{1}$ e $v \in U_{0} \cup U_{1}$. Assim, $\delta_{0}\left(u_{1}, x_{1}\right) D_{0}\left(\delta_{0}\left(u_{1}, x_{1}\right) v\right)=\delta_{0}\left(u_{1}, x_{1}\right) D_{0}(0)=0$ são válidas. Desse modo, 
usando os fatos

$$
\delta_{0}\left(U_{0}, \mathcal{A}_{0}\right)=\delta_{0}\left(U_{1}, \mathcal{A}_{0}\right)=0,
$$

como $\delta_{0}\left(u_{1}, x_{1}\right) \in \mathcal{A}_{0}$, seguem as igualdades

$$
\begin{aligned}
0 & =\delta_{0}\left(u_{1}, x_{1}\right) D_{0}\left(\delta_{0}\left(u_{1}, x_{1}\right) v\right) \\
& =\delta_{0}\left(u_{1}, x_{1}\right) D_{0}\left(\delta_{0}\left(u_{1}, x_{1}\right)\right) v+\delta_{0}\left(u_{1}, x_{1}\right)^{2} D_{0}(v) \\
& =\delta_{0}\left(u_{1}, x_{1}\right)^{2} D_{0}(v) .
\end{aligned}
$$

Consequentemente, pela definição da aplicação $\delta_{0}$, obtemos

$$
\delta_{0}\left(u_{1}, x_{1}\right)^{3}=\delta_{0}\left(u_{1}, x_{1}\right)^{2}\left(D_{0}\left(u_{1} x_{1}\right)-D_{0}\left(u_{1}\right) x_{1}-u_{1} D_{0}\left(x_{1}\right)=0,\right.
$$

pois $u_{1}, u_{1} x_{1} \in U_{0} \cup U_{1}$. Portanto, $\delta_{0}\left(u_{1}, x_{1}\right)$ é um elemento nilpotente que pertence ao centro de $\mathcal{A}_{0}$, por (i) do Lema 3.2.3, no caso $U=\mathcal{A}$. Assim, como $\mathcal{A}_{0}$ é uma álgebra semiprima, novamente pelo Lema 1.1.5, concluímos $\delta_{0}\left(u_{1}, x_{1}\right)=0$, para todos $u_{1} \in U_{1}, x_{1} \in \mathcal{A}_{1}$. Logo, estão provadas

$$
\delta_{0}\left(U_{0}, \mathcal{A}_{0}\right)=\delta_{0}\left(U_{0}, \mathcal{A}_{1}\right)=\delta_{0}\left(U_{1}, \mathcal{A}_{0}\right)=\delta_{0}\left(U_{1}, \mathcal{A}_{1}\right)=0
$$

o que nos permite concluir $\delta_{0}(U, \mathcal{A})=0$.

\subsubsection{Superderivações de Jordan de grau 1}

Nos próximos páragrafos, mantendo as notações fixadas no início da Seção 3.2, abordaremos o caso em que as superderivações de Jordan são de grau 1.

Lema 3.2.4. Se $D: \mathcal{A}_{0} \rightarrow \mathcal{A}_{1}$ é uma aplicação linear satisfazendo $D(x \circ y)=D(x) \circ y+x \circ D(y)$, para quaisquer $x, y \in \mathcal{A}_{0}$, então,

$$
[x, y] \perp(D(z w)-D(z) w-z D(w))
$$

para todos $x, y, z, w \in \mathcal{A}_{0}$.

Demonstração. Primeiramente, observe que pelo Lema 2.2.4, podemos concluir

$$
\begin{gathered}
{[x, y] \mathcal{A}_{0}[x, y] \mathcal{A}_{0}(D(x y)-D(x) y-x D(y))=0 \mathrm{e}} \\
(D(x y)-D(x) y-x D(y)) \mathcal{A}_{0}[x, y] \mathcal{A}_{0}[x, y]=0
\end{gathered}
$$

para quaisquer $x, y \in \mathcal{A}_{0}$. Denotando $c=[x, y]=c(x, y)$ e $m=D(x y)-D(x) y-x D(y)=m(x, y)$, as igualdades anteriores se resumem a $c \mathcal{A}_{0} c \mathcal{A}_{0} m=m \mathcal{A}_{0} c \mathcal{A}_{0} c=0$. Assim, dados $x_{0} \in \mathcal{A}_{0}$ e $x_{1} \in \mathcal{A}_{1}$, temos $\left(c x_{0} m\right) x_{1}\left(c x_{0} m\right)=c\left(x_{0} m x_{1}\right) c x_{0} m=0$, o que implica $c x_{0} m=0$, para todo $x_{0} \in \mathcal{A}_{0}$, por (i) do Lema 1.2.8. Analogamente, das igualdades $\left(m x_{0} c\right) x_{1}\left(m x_{0} c\right)=m x_{0} c\left(x_{1} m x_{0}\right) c=0$ resulta $m x_{0} c=0$, para todo $x_{0} \in \mathcal{A}_{0}$. Portanto, $c \in \mathcal{A}_{0}$ e $m \in \mathcal{A}_{1}$ são elementos satisfazendo

$$
c \mathcal{A}_{0} m=m \mathcal{A}_{0} c=0
$$

o que implica $c \mathcal{A} m=m \mathcal{A} c=0$, por (ii) do Lema 1.2.8, ou seja, $m \perp c$. Além disso, temos $c(x+$ $z, y)=c(x, y)+c(z, y), c(x, y+w)=c(x, y)+c(x, w), m(x+z, y)=m(x, y)+m(z, y), m(x, y+w)=$ 
$m(x, y)+m(x, w)$ e $c(x, y) a m(x, y)=m(x, y) a c(x, y)=0$, para quaisquer $x, y, z, w \in \mathcal{A}_{0}$ e $a \in \mathcal{A}$. Assim, linearizando esta última igualdade em $x$, obtemos $c(x, y) \operatorname{am}(z, y)+c(z, y) a m(x, y)=0$, isto é, $c(x, y) a m(z, y)=-c(z, y) a m(x, y)$, o que implica

$$
(c(x, y) a m(z, y)) b(c(x, y) a m(z, y))=-c(z, y)(a m(x, y) b c(x, y) a) m(z, y)=0,
$$

para todo $b \in \mathcal{A}$. Desse modo, como $\mathcal{A}$ é semiprima, pelo Lema 1.2 .4 , segue $c(x, y) \operatorname{am}(z, y)=0$, para todo $a \in \mathcal{A}$, cuja linearização em $y$ resulta em $c(x, y) \operatorname{am}(z, w)+c(x, w) a m(z, y)=0$, isto é, $c(x, y) a m(z, w)=-c(x, w) a m(z, y)$, o que implica

$$
(c(x, y) a m(z, w)) b(c(x, y) a m(z, w))=-c(x, w)(a m(z, y) b c(x, y) a) m(z, w)=0,
$$

para qualquer $b \in \mathcal{A}$, ou seja, $c(x, y) a m(z, w)=0$, para todos $x, y, z, w \in \mathcal{A}_{0}$ e $a \in \mathcal{A}$.

Teorema 3.2.2. (Fošner, [11]) Para a aplicação bilinear $\delta_{1}$, definida em (3.8), e o ideal $U$ de $\mathcal{A}$, definido no início da seção 3.2.1, a igualdade $\delta_{1}(U, \mathcal{A})=0$ é válida.

Demonstração. O primeiro passo é observar que, pelo Lema 3.2.4, temos $\left[\mathcal{A}_{0}, \mathcal{A}_{0}\right] \perp \delta_{1}\left(\mathcal{A}_{0}, \mathcal{A}_{0}\right)$, pois $\left.D_{1}\right|_{\mathcal{A}_{0}}$ satisfaz as hipóteses deste lema e $\delta_{1}\left(x_{0}, y_{0}\right)=D_{1}\left(x_{0} y_{0}\right)-D_{1}\left(x_{0}\right) y_{0}-x_{0} D_{1}\left(y_{0}\right)$, para quaisquer $x_{0}, y_{0} \in \mathcal{A}_{0}$. Em particular, ocorre

$$
\begin{aligned}
& {\left[y_{1} \delta_{1}\left(x_{0}, y_{0}\right), z_{0}\right] a\left[y_{1} \delta_{1}\left(x_{0}, y_{0}\right), z_{0}\right]} \\
& \quad=\left[y_{1} \delta_{1}\left(x_{0}, y_{0}\right), z_{0}\right]\left(a y_{1}\right) \delta_{1}\left(x_{0}, y_{0}\right) z_{0}-\left[y_{1} \delta_{1}\left(x_{0}, y_{0}\right), z_{0}\right]\left(a z_{0} y_{1}\right) \delta_{1}\left(x_{0}, y_{0}\right)=0
\end{aligned}
$$

para todo $a \in \mathcal{A}$, o que implica $\left[y_{1} \delta_{1}\left(x_{0}, y_{0}\right), z_{0}\right]=0$, quaisquer $x_{0}, y_{0}, z_{0} \in \mathcal{A}_{0}$ e $y_{1} \in \mathcal{A}_{1}$, pois $A$ é semiprima.

Analogamente, também obtemos que valem as igualdades

$$
\begin{aligned}
& {\left[\delta_{1}\left(x_{0}, y_{0}\right) y_{1}, z_{0}\right] a\left[\delta_{1}\left(x_{0}, y_{0}\right) y_{1}, z_{0}\right]} \\
& \quad=\left[\delta_{1}\left(x_{0}, y_{0}\right) y_{1}, z_{0}\right] a \delta_{1}\left(x_{0}, y_{0}\right) y_{1} z_{0}-\left[\delta_{1}\left(x_{0}, y_{0}\right) y_{1}, z_{0}\right]\left(a z_{0}\right) \delta_{1}\left(x_{0}, y_{0}\right) y_{1}=0
\end{aligned}
$$

para qualquer $a \in \mathcal{A}$, o que implica $\left[\delta_{1}\left(x_{0}, y_{0}\right) y_{1}, z_{0}\right]=0$, para todos $x_{0}, y_{0}, z_{0} \in \mathcal{A}_{0}$ e $y_{1} \in \mathcal{A}_{1}$. Assim, estão provadas as inclusões

$$
\mathcal{A}_{1} \delta_{1}\left(\mathcal{A}_{0}, \mathcal{A}_{0}\right), \delta_{1}\left(\mathcal{A}_{0}, \mathcal{A}_{0}\right) \mathcal{A}_{1} \subseteq Z\left(\mathcal{A}_{0}\right)
$$

Desse modo, do Lema 1.2.7, decorre

$$
\delta_{1}\left(x_{0}, y_{0}\right)^{2} \in Z(\mathcal{A}), \text { para todos } x_{0}, y_{0} \in \mathcal{A}_{0}
$$

Agora, observe que, dados $u_{0}, v_{0} \in U_{0}$, pela definição de $U$ e pelo Lema 3.2.4, podemos afirmar $\delta_{1}\left(u_{0}, y_{0}\right) y_{1} \perp v_{0}$, para quaisquer $y_{0} \in \mathcal{A}_{0}$ e $y_{1} \in \mathcal{A}_{1}$. Assim, temos $\delta_{1}\left(u_{0}, y_{0}\right) y_{1} \circ_{s} D_{1}\left(v_{0}\right)=0$, pelo Lema 3.2.1, isto é,

$$
\delta_{1}\left(u_{0}, y_{0}\right) y_{1} D_{1}\left(v_{0}\right)+D_{1}\left(v_{0}\right) \delta_{1}\left(u_{0}, y_{0}\right) y_{1}=0
$$

para quaisquer $y_{0} \in \mathcal{A}_{0}$ e $y_{1} \in \mathcal{A}_{1}$. Em particular, tomando $y_{1}=\delta_{1}\left(u_{0}, y_{0}\right) \in \mathcal{A}_{1}$, por (3.23), obtemos

$$
0=\delta_{1}\left(u_{0}, y_{0}\right)^{2} D_{1}\left(v_{0}\right)+D_{1}\left(v_{0}\right) \delta_{1}\left(u_{0}, y_{0}\right)^{2}=2 \delta_{1}\left(u_{0}, y_{0}\right)^{2} D_{1}\left(v_{0}\right)
$$


o que implica $\delta_{1}\left(u_{0}, y_{0}\right)^{2} D_{1}\left(v_{0}\right)=0$, para todo $v_{0} \in U_{0}$.

Além disso, pela definição de $\delta_{1}$, também temos

$$
\left(\delta_{1}\left(u_{0}, y_{0}\right) y_{1}\right)^{2}=\delta_{1}\left(u_{0}, y_{0}\right) y_{1}\left(D_{1}\left(u_{0} y_{0}\right)-D_{1}\left(u_{0}\right) y_{0}-u_{0} D_{1}\left(y_{0}\right)\right) y_{1},
$$

para todo $y_{i} \in \mathcal{A}_{i}$, onde $i=0,1$. Assim, a escolha de $y_{1}=\delta_{1}\left(u_{0}, y_{0}\right) \in \mathcal{A}_{1}$ resulta em

$$
\left(\delta_{1}\left(u_{0}, y_{0}\right)\right)^{4}=\delta_{1}\left(u_{0}, y_{0}\right)^{2}\left(D_{1}\left(u_{0} y_{0}\right)-D_{1}\left(u_{0}\right) y_{0}-u_{0} D_{1}\left(y_{0}\right)\right) \delta_{1}\left(u_{0}, y_{0}\right)=0
$$

pois $u_{0}, u_{0} y_{0} \in U_{0}$. Consequentemente, por (3.23), segue

$$
\delta_{1}\left(u_{0}, y_{0}\right)^{2} a \delta_{1}\left(u_{0}, y_{0}\right)^{2}=\delta_{1}\left(u_{0}, y_{0}\right)^{4} a=0
$$

para todo $a \in \mathcal{A}$, o que implica $\delta_{1}\left(u_{0}, y_{0}\right)^{2}=0$, para todos $u_{0} \in U_{0}$ e $y_{0} \in \mathcal{A}_{0}$, pela semiprimalidade de $\mathcal{A}$.

Prosseguindo, multiplicando (3.24) à esquerda por $\delta_{1}\left(u_{0}, y_{0}\right) y_{1}$, obtemos

$$
\begin{aligned}
0 & =\left(\delta_{1}\left(u_{0}, y_{0}\right) y_{1}\right)^{2} D_{1}\left(v_{0}\right)+\delta_{1}\left(u_{0}, y_{0}\right) y_{1}\left(D_{1}\left(v_{0}\right) \delta_{1}\left(u_{0}, y_{0}\right)\right) y_{1} \\
& =\left(\delta_{1}\left(u_{0}, y_{0}\right) y_{1}\right)^{2} D_{1}\left(v_{0}\right)+D_{1}\left(v_{0}\right) \delta_{1}\left(u_{0}, y_{0}\right)^{2} y_{1}^{2} \quad\left(\delta_{1}\left(\mathcal{A}_{0}, \mathcal{A}_{0}\right) \mathcal{A}_{1} \subseteq Z\left(\mathcal{A}_{0}\right)\right) \\
& =\left(\delta_{1}\left(u_{0}, y_{0}\right) y_{1}\right)^{2} D_{1}\left(v_{0}\right)
\end{aligned}
$$

Portanto, a multiplicação de (3.25) à esquerda por $\delta_{1}\left(u_{0}, y_{0}\right) y_{1}$ resulta em

$$
\left(\delta_{1}\left(u_{0}, y_{0}\right) y_{1}\right)^{3}=\left(\delta_{1}\left(u_{0}, y_{0}\right) y_{1}\right)^{2}\left(D_{1}\left(u_{0} y_{0}\right)-D_{1}\left(u_{0}\right) y_{0}-u_{0} D_{1}\left(y_{0}\right)\right) y_{1}=0 .
$$

Desse modo, $\delta_{1}\left(u_{0}, y_{0}\right) y_{1}$ é um elemento nilpotente que pertence ao centro da álgebra semiprima $\mathcal{A}_{0}$, o que implica $\delta_{1}\left(u_{0}, y_{0}\right) y_{1}=0$. Assim, também temos $\delta_{1}\left(u_{0}, y_{0}\right) y_{1} \delta_{1}\left(u_{0}, y_{0}\right)=0$, para todo $y_{1} \in \mathcal{A}_{1}$. Logo, como $\delta_{1}\left(u_{0}, y_{0}\right) \in \mathcal{A}_{1}$, por (i) do Lema 1.2.8 no caso $U=\mathcal{A}$, obtemos $\delta_{1}\left(u_{0}, y_{0}\right)=0$, para todos $u_{0} \in U_{0}$ e $y_{0} \in \mathcal{A}_{0}$, isto é,

$$
\delta_{1}\left(U_{0}, \mathcal{A}_{0}\right)=0
$$

Agora, por (vii) do Lema 3.2.3, seguem as igualdades

$$
\begin{aligned}
0= & \delta_{1}\left(u_{0}, x_{1}\right) y_{1}\left[x_{0}, y_{0} v_{0}\right] \\
= & \delta_{1}\left(u_{0}, x_{1}\right) y_{1} x_{0} y_{0} v_{0}-\delta_{1}\left(u_{0}, x_{1}\right) y_{1} y_{0} v_{0} x_{0} \\
= & \delta_{1}\left(u_{0}, x_{1}\right) y_{1} x_{0} y_{0} v_{0}-\delta_{1}\left(u_{0}, x_{1}\right) y_{1} y_{0} x_{0} v_{0}+\delta_{1}\left(u_{0}, x_{1}\right) y_{1} y_{0} x_{0} v_{0} \\
& -\delta_{1}\left(u_{0}, x_{1}\right) y_{1} y_{0} v_{0} x_{0} \\
= & \delta_{1}\left(u_{0}, x_{1}\right) y_{1}\left[x_{0}, y_{0}\right] v_{0}+\delta_{1}\left(u_{0}, x_{1}\right) y_{1} y_{0}\left[x_{0}, v_{0}\right] \\
= & \delta_{1}\left(u_{0}, x_{1}\right) y_{1}\left[x_{0}, y_{0}\right] v_{0},
\end{aligned}
$$

para todos $u_{0}, v_{0} \in U_{0}, x_{0}, y_{0} \in \mathcal{A}_{0}$ e $x_{1}, y_{1} \in \mathcal{A}_{1}$, ou seja, $\delta_{1}\left(u_{0}, x_{1}\right) y_{1}\left[x_{0}, y_{0}\right] U_{0}=0$ 
Analogamente, novamente por (vii) do Lema 3.2.3, temos

$$
\begin{aligned}
0= & {\left[v_{0} x_{0}, y_{0}\right] y_{1} \delta_{1}\left(u_{0}, x_{1}\right) } \\
= & v_{0} x_{0} y_{0} y_{1} \delta_{1}\left(u_{0}, x_{1}\right)-y_{0} v_{0} x_{0} y_{1} \delta_{1}\left(u_{0}, x_{1}\right) \\
= & v_{0} x_{0} y_{0} y_{1} \delta_{1}\left(u_{0}, x_{1}\right)-v_{0} y_{0} x_{0} y_{1} \delta_{1}\left(u_{0}, x_{1}\right)+v_{0} y_{0} x_{0} y_{1} \delta_{1}\left(u_{0}, x_{1}\right) \\
& -y_{0} v_{0} x_{0} y_{1} \delta_{1}\left(u_{0}, x_{1}\right) \\
= & v_{0}\left[x_{0}, y_{0}\right] y_{1} \delta_{1}\left(u_{0}, x_{1}\right)+\left[v_{0}, y_{0}\right] x_{0} y_{1} \delta_{1}\left(u_{0}, x_{1}\right) \\
= & v_{0}\left[x_{0}, y_{0}\right] y_{1} \delta_{1}\left(u_{0}, x_{1}\right),
\end{aligned}
$$

para quaisquer $u_{0}, v_{0} \in U_{0}, x_{0}, y_{0} \in \mathcal{A}_{0}$ e $x_{1}, y_{1} \in \mathcal{A}_{1}$, isto é, $U_{0}\left[x_{0}, y_{0}\right] y_{1} \delta_{1}\left(u_{0}, x_{1}\right)=0$.

Assim, em decorrência da pertinência $\delta_{1}\left(u_{0}, x_{1}\right) y_{1}\left[x_{0}, y_{0}\right],\left[x_{0}, y_{0}\right] y_{1} \delta_{1}\left(u_{0}, x_{1}\right) \in U_{1}$ e por (iii) do Lema 1.2.8, segue

$$
\delta_{1}\left(U_{0}, \mathcal{A}_{1}\right) \mathcal{A}_{1}\left[\mathcal{A}_{0}, \mathcal{A}_{0}\right]=\left[\mathcal{A}_{0}, \mathcal{A}_{0}\right] \mathcal{A}_{1} \delta_{1}\left(U_{0}, \mathcal{A}_{1}\right)=0,
$$

Desse modo, usando (i), (ii) e (iv) do Lema 3.2.3, temos as igualdades

$$
\begin{aligned}
\delta_{1}\left(u_{0}, x_{1}\right) y_{1}-y_{1} \delta_{1}\left(u_{0}, x_{1}\right) & =u_{0} \delta_{1}\left(x_{1}, y_{1}\right)-\delta_{1}\left(u_{0} x_{1}, y_{1}\right)+\delta_{1}\left(x_{1} u_{0}, y_{1}\right)-\delta_{1}\left(x_{1}, y_{1}\right) u_{0} \\
& =-\left[\delta_{1}\left(x_{1}, y_{1}\right), u_{0}\right]+\delta_{1}\left(x_{1} u_{0}, y_{1}\right)-\delta_{1}\left(u_{0} x_{1}, y_{1}\right) \\
& =\delta_{1}\left(x_{1} u_{0}, y_{1}\right)-\delta_{1}\left(u_{0} x_{1}, y_{1}\right)
\end{aligned}
$$

para todos $u_{0} \in U_{0}$ e $x_{1}, y_{1} \in \mathcal{A}_{1}$. Portanto, para qualquer $v_{0} \in U_{0}$, temos

$$
\left[\delta_{1}\left(u_{0}, x_{1}\right) y_{1}, v_{0}\right]-\left[y_{1} \delta_{1}\left(u_{0}, x_{1}\right), v_{0}\right]=\left[\delta_{1}\left(x_{1} u_{0}, y_{1}\right), v_{0}\right]-\left[\delta_{1}\left(u_{0} x_{1}, y_{1}\right), v_{0}\right]=0
$$

pois $x_{1} u_{0}, u_{0} x_{1} \in \mathcal{A}_{1}$, ou seja, $\left[\delta_{1}\left(u_{0}, x_{1}\right) y_{1}, v_{0}\right]=\left[y_{1} \delta_{1}\left(u_{0}, x_{1}\right), v_{0}\right]$, para todos $u_{0}, v_{0} \in U_{0}$ e $x_{1}, y_{1} \in \mathcal{A}_{1}$, que multiplicando à direita por $z_{1}\left[z_{0}, y_{0}\right]$, resulta em $\left[\delta_{1}\left(u_{0}, x_{1}\right) y_{1}, v_{0}\right] z_{1}\left[z_{0}, y_{0}\right]=$ $\left[y_{1} \delta_{1}\left(u_{0}, x_{1}\right), v_{0}\right] z_{1}\left[z_{0}, y_{0}\right]=0$, para quaisquer $z_{1} \in \mathcal{A}_{1}$ e $z_{0}, y_{0} \in \mathcal{A}_{0}$, por (3.27). Logo, os elementos $\left[\delta_{1}\left(u_{0}, x_{1}\right) y_{1}, v_{0}\right] \in \mathcal{A}_{1}$ e $\left[z_{0}, y_{0}\right] \in \mathcal{A}_{0}$ são tais que

$$
\left[\delta_{1}\left(u_{0}, x_{1}\right) y_{1}, v_{0}\right] \mathcal{A}_{1}\left[z_{0}, y_{0}\right]=\left[y_{1} \delta_{1}\left(u_{0}, x_{1}\right), v_{0}\right] \mathcal{A}_{1}\left[z_{0}, y_{0}\right]=0
$$

o que, por (ii) do Lema 1.2.8, implica

$$
\left[\delta_{1}\left(u_{0}, x_{1}\right) y_{1}, v_{0}\right] \mathcal{A}\left[z_{0}, y_{0}\right]=\left[y_{1} \delta_{1}\left(u_{0}, x_{1}\right), v_{0}\right] \mathcal{A}\left[z_{0}, y_{0}\right]=0
$$

Em particular, também temos

$$
\begin{aligned}
& {\left[\delta_{1}\left(u_{0}, x_{1}\right) y_{1}, v_{0}\right] a\left[\delta_{1}\left(u_{0}, x_{1}\right) y_{1}, v_{0}\right]=} \\
& {\left[\delta_{1}\left(u_{0}, x_{1}\right) y_{1}, v_{0}\right]\left(a \delta_{1}\left(u_{0}, x_{1}\right) y_{1}\right) v_{0}-\left[\delta_{1}\left(u_{0}, x_{1}\right) y_{1}, v_{0}\right] a v_{0} \delta_{1}\left(u_{0}, x_{1}\right) y_{1}=0 \mathrm{e}} \\
& {\left[y_{1} \delta_{1}\left(u_{0}, x_{1}\right), v_{0}\right] a\left[y_{1} \delta_{1}\left(u_{0}, x_{1}\right), v_{0}\right]=} \\
& {\left[y_{1} \delta_{1}\left(u_{0}, x_{1}\right), v_{0}\right]\left(a y_{1} \delta_{1}\left(u_{0}, x_{1}\right)\right) v_{0}-\left[y_{1} \delta_{1}\left(u_{0}, x_{1}\right), v_{0}\right] a v_{0} y_{1} \delta_{1}\left(u_{0}, x_{1}\right)=0,}
\end{aligned}
$$

para todo $a \in \mathcal{A}$, pois $v_{0} \in U=\operatorname{ideal}\left\langle\left[\mathcal{A}_{0}, \mathcal{A}_{0}\right]\right\rangle$, de onde decorre

$$
\left[\delta_{1}\left(u_{0}, x_{1}\right) y_{1}, v_{0}\right]=\left[y_{1} \delta_{1}\left(u_{0}, x_{1}\right), v_{0}\right]=0
$$

para todos $u_{0}, v_{0} \in U_{0}, x_{1}, y_{1} \in \mathcal{A}_{1}$, pela semiprimalidade de $\mathcal{A}$. Consequentemente, por (ii) e (iv) 
do Lema 3.2.3, obtemos as igualdades $\left[u_{0} \delta_{1}\left(x_{1}, y_{1}\right), v_{0}\right]=\left[\delta_{1}\left(u_{0}, x_{1}\right) y_{1}, v_{0}\right]+\left[\delta_{1}\left(u_{0} x_{1}, y_{1}\right), v_{0}\right]=0$ e $\left[\delta_{1}\left(x_{1}, y_{1}\right) u_{0}, v_{0}\right]=\left[\delta_{1}\left(x_{1} u_{0}, y_{1}\right), v_{0}\right]+\left[y_{1} \delta_{1}\left(u_{0}, x_{1}\right), v_{0}\right]=0$, para todos $u_{0}, v_{0} \in U_{0}, x_{1}, y_{1} \in \mathcal{A}_{1}$, isto é, são válidas

$$
\left[U_{0} \delta_{1}\left(\mathcal{A}_{1}, \mathcal{A}_{1}\right), U_{0}\right]=\left[\delta_{1}\left(\mathcal{A}_{1}, \mathcal{A}_{1}\right) U_{0}, U_{0}\right]=0 .
$$

Desse modo, como $\delta_{1}\left(\mathcal{A}_{1}, \mathcal{A}_{1}\right) \subseteq \mathcal{A}_{1}$, concluímos $U_{0} \delta_{1}\left(\mathcal{A}_{1}, \mathcal{A}_{1}\right) \subseteq Z(\mathcal{A})$, pelo Lema 1.2.9.

Agora, por (3.27) e por (i) do Lema 3.2.3, temos

$$
\begin{aligned}
0 & =\left[u_{0} x_{1}, v_{0}\right] \\
& =u_{0} x_{1} v_{0}-v_{0} u_{0} x_{1} \\
& =u_{0} x_{1} v_{0}-u_{0} v_{0} x_{1}+u_{0} v_{0} x_{1}-v_{0} u_{0} x_{1} \\
& =u_{0}\left[x_{1}, v_{0}\right]+\left[u_{0}, v_{0}\right] x_{1} \\
& =\left[u_{0}, v_{0}\right] x_{1}
\end{aligned}
$$

para todos $u_{0}, v_{0} \in U_{0}$ e $x_{1} \in \delta_{1}\left(\mathcal{A}_{1}, \mathcal{A}_{1}\right)$, isto é, $\left[U_{0}, U_{0}\right] \delta_{1}\left(\mathcal{A}_{1}, \mathcal{A}_{1}\right)=0$.

Similarmente, também valem

$$
\begin{aligned}
0 & =\left[x_{1} u_{0}, v_{0}\right] \\
& =x_{1} u_{0} v_{0}-v_{0} x_{1} u_{0} \\
& =x_{1} u_{0} v_{0}-x_{1} v_{0} u_{0}+x_{1} v_{0} u_{0}-v_{0} x_{1} u_{0} \\
& =x_{1}\left[u_{0}, v_{0}\right]+\left[x_{1}, v_{0}\right] u_{0} \\
& =x_{1}\left[u_{0}, v_{0}\right]
\end{aligned}
$$

para quaisquer $u_{0}, v_{0} \in U_{0}$ e $x_{1} \in \delta_{1}\left(\mathcal{A}_{1}, \mathcal{A}_{1}\right)$, ou seja, $\delta_{1}\left(\mathcal{A}_{1}, \mathcal{A}_{1}\right)\left[U_{0}, U_{0}\right]=0$.

Desse modo, pelo Lema 1.2.10, a igualdade $\delta_{1}\left(\mathcal{A}_{1}, \mathcal{A}_{1}\right) U=0$ é satisfeita, e consequentemente segue

$$
\delta_{1}\left(\mathcal{A}_{1}, \mathcal{A}_{1}\right) \perp\left[\mathcal{A}_{0}, \mathcal{A}_{0}\right] .
$$

Em particular, $\left[\delta_{1}\left(\mathcal{A}_{1}, \mathcal{A}_{1}\right) \mathcal{A}_{1}, \mathcal{A}_{0}\right] \mathcal{A}\left[\delta_{1}\left(\mathcal{A}_{1}, \mathcal{A}_{1}\right) \mathcal{A}_{1}, \mathcal{A}_{0}\right]=0$, pois $\delta_{1}\left(\mathcal{A}_{1}, \mathcal{A}_{1}\right) \mathcal{A}_{1} \subseteq \mathcal{A}_{0}$, o que implica $\left[\delta_{1}\left(\mathcal{A}_{1}, \mathcal{A}_{1}\right) \mathcal{A}_{1}, \mathcal{A}_{0}\right]=0$, pois $\mathcal{A}$ é semiprima, ou seja, $\delta_{1}\left(\mathcal{A}_{1}, \mathcal{A}_{1}\right) \mathcal{A}_{1} \subseteq Z\left(\mathcal{A}_{0}\right)$. Analogamente, $\left[\mathcal{A}_{1} \delta_{1}\left(\mathcal{A}_{1}, \mathcal{A}_{1}\right), \mathcal{A}_{0}\right] \mathcal{A}\left[\mathcal{A}_{1} \delta_{1}\left(\mathcal{A}_{1}, \mathcal{A}_{1}\right), \mathcal{A}_{0}\right]=0$ nos leva a $\left[\mathcal{A}_{1} \delta_{1}\left(\mathcal{A}_{1}, \mathcal{A}_{1}\right), \mathcal{A}_{0}\right]=0$, isto é, $\mathcal{A}_{1} \delta_{1}\left(\mathcal{A}_{1}, \mathcal{A}_{1}\right) \subseteq Z\left(\mathcal{A}_{0}\right)$. Assim, aplicando o Lema 1.2 .7 concluímos

$$
\delta_{1}\left(x_{1}, y_{1}\right)^{2} \in Z(\mathcal{A}), \text { para todos } x_{1}, y_{1} \in \mathcal{A}_{1}
$$

Se $u_{1} \in U_{1}, v \in U_{0} \cup U_{1}$ e $x_{1}, y_{1} \in \mathcal{A}_{1}$, então, por (3.29) e pela definição de $U$, temos $\delta_{1}\left(u_{1}, x_{1}\right) y_{1} \perp v$, o que implica $\delta_{1}\left(u_{1}, x_{1}\right) y_{1} \circ_{s} D_{1}(v)=0$, pelo Lema 3.2.1, ou seja,

$$
\delta_{1}\left(u_{1}, x_{1}\right) y_{1} D_{1}(v)+D_{1}(v) \delta_{1}\left(u_{1}, x_{1}\right) y_{1}=0 .
$$

Além disso, por (3.30), a substituição $y_{1}=\delta_{1}\left(u_{1}, x_{1}\right) \in \mathcal{A}_{1}$, resulta em $\delta_{1}\left(u_{1}, x_{1}\right)^{2} D_{1}(v)=0$, e pela definição da aplicação $\delta_{1}$, obtemos

$$
\left(\delta_{1}\left(u_{1}, x_{1}\right) y_{1}\right)^{2}=\delta_{1}\left(u_{1}, x_{1}\right) y_{1}\left(D_{1}\left(u_{1} x_{1}\right)-D_{1}\left(u_{1}\right) x_{1}+u_{1} D_{1}\left(x_{1}\right)\right) y_{1},
$$


Assim, novamente tomando $y_{1}=\delta_{1}\left(u_{1}, x_{1}\right)$, seguem

$$
\left(\delta_{1}\left(u_{1}, x_{1}\right)\right)^{4}=\delta_{1}\left(u_{1}, x_{1}\right)^{2}\left(D_{1}\left(u_{1} x_{1}\right)-D_{1}\left(u_{1}\right) x_{1}+u_{1} D_{1}\left(x_{1}\right)\right) \delta_{1}\left(u_{1}, x_{1}\right)=0,
$$

pois $u_{1} x_{1}, u_{1} \in U_{0} \cup U_{1}$. Portanto, por (3.30), obtemos $\delta_{1}\left(u_{1}, x_{1}\right)^{2} a \delta_{1}\left(u_{1}, x_{1}\right)^{2}=\delta_{1}\left(u_{1}, x_{1}\right)^{4} a=0$, para todo $a \in \mathcal{A}$, o que implica $\delta_{1}\left(u_{1}, x_{1}\right)^{2}=0$, para todos $u_{1} \in U_{1}, x_{1} \in \mathcal{A}_{1}$, pois $\mathcal{A}$ é semiprima.

Se $v \in U_{0}$, então, multiplicando a equação (3.31) à esquerda por $\delta_{1}\left(u_{1}, x_{1}\right) y_{1}$, obtemos

$$
\begin{aligned}
0 & =\left(\delta_{1}\left(u_{1}, x_{1}\right) y_{1}\right)^{2} D_{1}(v)+\delta_{1}\left(u_{1}, x_{1}\right) y_{1}\left(D_{1}(v) \delta_{1}\left(u_{1}, x_{1}\right)\right) y_{1} \\
& =\left(\delta_{1}\left(u_{1}, x_{1}\right) y_{1}\right)^{2} D_{1}(v)+D_{1}(v) \delta_{1}\left(u_{1}, x_{1}\right)^{2} y_{1}^{2} \\
& =\left(\delta_{1}\left(u_{1}, x_{1}\right) y_{1}\right)^{2} D_{1}(v)
\end{aligned}
$$

pois $\delta_{1}\left(\mathcal{A}_{1}, \mathcal{A}_{1}\right) \mathcal{A}_{1} \subseteq Z\left(\mathcal{A}_{0}\right)$. Assim, também por esta última inclusão, se $v \in U_{1}$, então, $D_{1}(v) \in \mathcal{A}_{0}$, e por $(3.31)$, concluímos $\delta_{1}\left(u_{1}, x_{1}\right) y_{1} D_{1}(v)=0$.

Agora, multiplicando a equação (3.32) à esquerda por $\delta_{1}\left(u_{1}, x_{1}\right) y_{1}$, chegamos a

$$
\left.\left(\delta_{1}\left(u_{1}, x_{1}\right) y_{1}\right)^{3}=\left(\delta_{1}\left(u_{1}, x_{1}\right) y_{1}\right)^{2}\left(D_{1}\left(u_{1} x_{1}\right)-D_{1}\left(u_{1}\right)\right) x_{1}+u_{1} D_{1}\left(x_{1}\right)\right) y_{1}=0 .
$$

Desse modo, como $\delta_{1}\left(u_{1}, x_{1}\right) y_{1}$ é um elemento nilpotente pertencente ao centro da álgebra semiprima $\mathcal{A}_{0}$, temos $\delta_{1}\left(u_{1}, x_{1}\right) y_{1}=0$, para todos $u_{1} \in U_{1}$ e $x_{1}, y_{1} \in \mathcal{A}_{1}$. Portanto, $\delta_{1}\left(u_{1}, x_{1}\right) \in \mathcal{A}_{1}$ é um elemento satisfazendo $\delta_{1}\left(u_{1}, x_{1}\right) \mathcal{A}_{1} \delta_{1}\left(u_{1}, x_{1}\right)=0$, o que implica $\delta_{1}\left(u_{1}, x_{1}\right)=0$, para todos $u_{1} \in U_{1}$ e $x_{1} \in \mathcal{A}_{1}$, por (i) do Lema 1.2 .8 no caso $U=\mathcal{A}$. Logo, provamos que a seguinte igualdade é válida.

$$
\delta_{1}\left(U_{1}, \mathcal{A}_{1}\right)=0
$$

Além disso, por (iii) do Lema 3.2.3, temos $\delta_{1}\left(x_{0}, u_{1}\right) x_{1}=x_{0} \delta_{1}\left(u_{1}, x_{1}\right)-\delta_{1}\left(x_{0} u_{1}, x_{1}\right)=0$, para todos $x_{0} \in \mathcal{A}_{0}, u_{1} \in U_{1}$ e $x_{1} \in \mathcal{A}_{1}$. Assim, para qualquer $a=a_{0}+a_{1} \in \mathcal{A}$, obtemos $\delta_{1}\left(x_{0}, u_{1}\right) a x_{1}=\delta_{1}\left(x_{0}, u_{1}\right)\left(a_{0} x_{1}\right)+\left(\delta_{1}\left(x_{0}, u_{1}\right) a_{1}\right) x_{1}=0$, isto é,

$$
\delta_{1}\left(x_{0}, u_{1}\right) \perp x_{1} \text {, para todos } x_{0} \in \mathcal{A}_{0}, u_{1} \in U_{1} \text { e } x_{1} \in \mathcal{A}_{1},
$$

o que implica $\delta_{1}\left(x_{0}, u_{1}\right) \circ_{s} D_{1}\left(x_{1}\right)=0$, pelo Lema 3.2.1, ou seja,

$$
\delta_{1}\left(x_{0}, u_{1}\right) D_{1}\left(x_{1}\right)+D_{1}\left(x_{1}\right) \delta_{1}\left(x_{0}, u_{1}\right)=0
$$

para quaisquer $x_{0} \in \mathcal{A}_{0}, x_{1} \in \mathcal{A}_{1}$ e $u_{1} \in U_{1}$.

Dado $y_{0} \in \mathcal{A}_{0}$, para todo $a=a_{0}+a_{1} \in \mathcal{A}$, temos $\delta_{1}\left(x_{0}, u_{1}\right) y_{0} a x_{1}=\delta_{1}\left(x_{0}, u_{1}\right)\left(y_{0} a_{0} x_{1}\right)+$ $\left(\delta_{1}\left(x_{0}, u_{1}\right) y_{0} a_{1}\right) x_{1}=0$, o que nos permite concluir $\delta_{1}\left(x_{0}, u_{1}\right) y_{0} \perp x_{1}$, e novamente pelo Lema 3.2.1, obtemos

$$
\delta_{1}\left(x_{0}, u_{1}\right) y_{0} D_{1}\left(x_{1}\right)+D_{1}\left(x_{1}\right) \delta_{1}\left(x_{0}, u_{1}\right) y_{0}=0
$$

para todo $y_{0} \in \mathcal{A}_{0}$.

Agora, multiplicando (3.35) à direita por $y_{0}$, temos $\delta_{1}\left(x_{0}, u_{1}\right) D_{1}\left(x_{1}\right) y_{0}+D_{1}\left(x_{1}\right) \delta_{1}\left(x_{0}, u_{1}\right) y_{0}=$ 0 , e subtraindo esta igualdade de (3.36), são válidas $\delta_{1}\left(x_{0}, u_{1}\right) y_{0} D_{1}\left(x_{1}\right)-\delta_{1}\left(x_{0}, u_{1}\right) D_{1}\left(x_{1}\right) y_{0}=$ $\delta_{1}\left(x_{0}, u_{1}\right)\left[y_{0}, D_{1}\left(x_{1}\right)\right]=0$, para todos $x_{0}, y_{0} \in \mathcal{A}_{0}, u_{1} \in U_{1}$ e $x_{1} \in \mathcal{A}_{1}$. Desse modo, como 
$\delta_{1}\left(x_{0}, u_{1}\right), D_{1}\left(x_{1}\right) \in \mathcal{A}_{0}$, aplicando o Lema 1.2.11, concluímos $\left[\delta_{1}\left(x_{0}, u_{1}\right), D_{1}\left(x_{1}\right)\right]=0$. Portanto,

$$
\begin{aligned}
{\left[\delta_{1}\left(x_{0}, u_{1}\right) y_{0}, D_{1}\left(x_{1}\right)\right]=} & \delta_{1}\left(x_{0}, u_{1}\right) y_{0} D_{1}\left(x_{1}\right)-D_{1}\left(x_{1}\right) \delta_{1}\left(x_{0}, u_{1}\right) y_{0} \\
= & \delta_{1}\left(x_{0}, u_{1}\right) y_{0} D_{1}\left(x_{1}\right)-\delta_{1}\left(x_{0}, u_{1}\right) D_{1}\left(x_{1}\right) y_{0}+\delta_{1}\left(x_{0}, u_{1}\right) D_{1}\left(x_{1}\right) y_{0} \\
& -D_{1}\left(x_{1}\right) \delta_{1}\left(x_{0}, u_{1}\right) y_{0} \\
= & \delta_{1}\left(x_{0}, u_{1}\right)\left[y_{0}, D_{1}\left(x_{1}\right)\right]+\left[\delta_{1}\left(x_{0}, u_{1}\right), D_{1}\left(x_{1}\right)\right] y_{0}=0,
\end{aligned}
$$

o que resulta, por (3.36), em $\delta_{1}\left(x_{0}, u_{1}\right) y_{0} D_{1}\left(x_{1}\right)=0$, para todos $x_{0}, y_{0} \in \mathcal{A}_{0}, u_{1} \in U_{1}$ e $x_{1} \in \mathcal{A}_{1}$. Assim, esta última igualdade e (3.34) implicam

$$
\delta_{1}\left(x_{0}, u_{1}\right) y_{0} \delta_{1}\left(x_{0}, u_{1}\right)=\delta_{1}\left(x_{0}, u_{1}\right) y_{0}\left(D_{1}\left(x_{0} u_{1}\right)-D_{1}\left(x_{0}\right) u_{1}-x_{0} D_{1}\left(u_{1}\right)\right)=0,
$$

o que nos permite concluir $\delta_{1}\left(x_{0}, u_{1}\right)=0$, para todos $x_{0} \in \mathcal{A}_{0}$ e $u_{1} \in U_{1}$, isto é,

$$
\delta_{1}\left(\mathcal{A}_{0}, U_{1}\right)=0
$$

Prosseguindo, por (ii) e (iv) do Lema 3.2 .3 e por (3.33), seguem

$$
\begin{aligned}
& \delta_{1}\left(u_{0}, x_{1}\right) u_{1}=u_{0} \delta_{1}\left(x_{1}, u_{1}\right)-\delta_{1}\left(u_{0} x_{1}, u_{1}\right)=0 \mathrm{e} \\
& u_{1} \delta_{1}\left(u_{0}, x_{1}\right)=-\delta_{1}\left(x_{1} u_{0}, u_{1}\right)+\delta_{1}\left(x_{1}, u_{1}\right) u_{0}=0,
\end{aligned}
$$

para todos $u_{0} \in U_{0}, u_{1} \in U_{1}$ e $x_{1} \in \mathcal{A}_{1}$. Portanto, temos $\delta_{1}\left(U_{0}, \mathcal{A}_{1}\right) \mathcal{A}_{0} U_{1}=U_{1} \mathcal{A}_{0} \delta_{1}\left(U_{0}, \mathcal{A}_{1}\right)=0$, pois $\mathcal{A}_{0} U_{1}, U_{1} \mathcal{A}_{0} \subseteq U \cap \mathcal{A}_{1}=U_{1}$. Desse modo, por (ii) do Lema 1.2 .8 , no caso $U=\mathcal{A}$, valem $\delta_{1}\left(U_{0}, \mathcal{A}_{1}\right) \mathcal{A} U_{1}=U_{1} \mathcal{A} \delta_{1}\left(U_{0}, \mathcal{A}_{1}\right)=0$, pois $\delta_{1}\left(U_{0}, \mathcal{A}_{1}\right) \subseteq \mathcal{A}_{0}$ e $U_{1} \subseteq \mathcal{A}_{1}$, isto é,

$$
\delta_{1}\left(U_{0}, \mathcal{A}_{1}\right) \perp U_{1}
$$

Assim, dados $u_{0} \in U_{0}$ e $u_{1} \in U_{1}$, temos $\delta_{1}\left(u_{0}, x_{1}\right) y_{0} \perp u_{1}$, para todos $y_{0} \in \mathcal{A}_{0}$ e $x_{1} \in \mathcal{A}_{1}$, o que implica

$$
0=D_{1}\left(\left(\delta_{1}\left(u_{0}, x_{1}\right) y_{0}\right) u_{1}\right)=D_{1}\left(\delta_{1}\left(u_{0}, x_{1}\right) y_{0}\right) u_{1}+\delta_{1}\left(u_{0}, x_{1}\right) y_{0} D_{1}\left(u_{1}\right)
$$

por (3.37). Logo, multiplicando esta igualdade à esquerda por $\delta_{1}\left(u_{0}, x_{1}\right) z_{0}$, onde $z_{0} \in \mathcal{A}_{0}$, obtemos

$$
\begin{aligned}
0 & =\delta_{1}\left(u_{0}, x_{1}\right)\left(z_{0} D_{1}\left(\delta_{1}\left(u_{0}, x_{1}\right) y_{0}\right)\right) u_{1}+\delta_{1}\left(u_{0}, x_{1}\right) z_{0} \delta_{1}\left(u_{0}, x_{1}\right) y_{0} D_{1}\left(u_{1}\right) \\
& =\delta_{1}\left(u_{0}, x_{1}\right) z_{0} \delta_{1}\left(u_{0}, x_{1}\right) y_{0} D_{1}\left(u_{1}\right) .
\end{aligned}
$$

Portanto, $\left(\delta_{1}\left(u_{0}, x_{1}\right) y_{0} D_{1}\left(u_{1}\right)\right) \mathcal{A}_{0}\left(\delta_{1}\left(u_{0}, x_{1}\right) y_{0} D_{1}\left(u_{1}\right)\right)=0$, para todos $u_{0} \in U_{0}, u_{1} \in U_{1}, y_{0} \in$ $\mathcal{A}_{0}$ e $x_{1} \in \mathcal{A}_{1}$, pois $y_{0} D_{1}\left(u_{1}\right) \mathcal{A}_{0} \subseteq \mathcal{A}_{0}$, de onde resulta

$$
\delta_{1}\left(U_{0}, \mathcal{A}_{1}\right) \mathcal{A}_{0} D_{1}\left(U_{1}\right)=0
$$

pois $\delta_{1}\left(U_{0}, \mathcal{A}_{1}\right) \mathcal{A}_{0} D_{1}\left(U_{1}\right) \subseteq \mathcal{A}_{0}$ e $\mathcal{A}_{0}$ é uma álgebra semiprima.

Agora, por (3.38), temos $\delta_{1}\left(u_{0}, x_{1}\right) v_{0} a y_{1}=\delta_{1}\left(u_{0}, x_{1}\right)\left(v_{0} a_{0} y_{1}\right)+\left(\delta_{1}\left(u_{0}, x_{1}\right) v_{0} a_{1}\right) y_{1}=0$, para todo $a \in \mathcal{A}$, isto é, $\delta_{1}\left(u_{0}, x_{1}\right) v_{0} \perp y_{1}$, para quaisquer $u_{0}, v_{0} \in U_{0}$ e $x_{1}, y_{1} \in \mathcal{A}_{1}$. Assim, aplicando o Lema 3.2.1, obtemos $\delta_{1}\left(u_{0}, x_{1}\right) v_{0} \circ_{s} D_{1}\left(y_{1}\right)=0$, ou seja,

$$
\delta_{1}\left(u_{0}, x_{1}\right) v_{0} D_{1}\left(y_{1}\right)+D_{1}\left(y_{1}\right) \delta_{1}\left(u_{0}, x_{1}\right) v_{0}=0 .
$$


Analogamente, dado $x_{0} \in \mathcal{A}_{0}$, vale $x_{0} \delta_{1}\left(u_{0}, x_{1}\right) v_{0} \perp y_{1}$, e obtemos

$$
x_{0} \delta_{1}\left(u_{0}, x_{1}\right) v_{0} D_{1}\left(y_{1}\right)+D_{1}\left(y_{1}\right) x_{0} \delta_{1}\left(u_{0}, x_{1}\right) v_{0}=0 .
$$

Além disso, a multiplicação de (3.40) por $x_{0}$ à esquerda, resulta em

$$
x_{0} \delta_{1}\left(u_{0}, x_{1}\right) v_{0} D_{1}\left(y_{1}\right)+x_{0} D_{1}\left(y_{1}\right) \delta_{1}\left(u_{0}, x_{1}\right) v_{0}=0
$$

que comparando com (3.41), nos permite concluir $\left[x_{0}, D_{1}\left(y_{1}\right)\right] \delta_{1}\left(u_{0}, x_{1}\right) v_{0}=0$, para todos $u_{0}, v_{0} \in$ $U_{0}, x_{0} \in \mathcal{A}_{0}$ e $x_{1}, y_{1} \in \mathcal{A}_{1}$. Logo, a seguinte igualdade é válida

$$
\left(\left[\mathcal{A}_{0}, D_{1}\left(\mathcal{A}_{1}\right)\right] \delta_{1}\left(U_{0}, \mathcal{A}_{1}\right)\right) U_{0}\left(\left[\mathcal{A}_{0}, D_{1}\left(\mathcal{A}_{1}\right)\right] \delta_{1}\left(U_{0}, \mathcal{A}_{1}\right)\right)=0
$$

o que implica $\left[\mathcal{A}_{0}, D_{1}\left(\mathcal{A}_{1}\right)\right] \delta_{1}\left(U_{0}, \mathcal{A}_{1}\right)=0$, pois $\left[\mathcal{A}_{0}, D_{1}\left(\mathcal{A}_{1}\right)\right] \delta_{1}\left(U_{0}, \mathcal{A}_{1}\right) \subseteq U_{0}$, pela definição de $U$, e $U_{0}$ é uma álgebra semiprima. Do mesmo modo, também temos $\delta_{1}\left(u_{0}, x_{1}\right) v_{0} x_{0} \perp y_{1}$, de onde obtemos

$$
\delta_{1}\left(u_{0}, x_{1}\right) v_{0} x_{0} D_{1}\left(y_{1}\right)+D_{1}\left(y_{1}\right) \delta_{1}\left(u_{0}, x_{1}\right) v_{0} x_{0}=0 .
$$

Prosseguindo, observe que a multiplicação de (3.40) à direita por $x_{0}$, nos leva a

$$
\delta_{1}\left(u_{0}, x_{1}\right) v_{0} D_{1}\left(y_{1}\right) x_{0}+D_{1}\left(y_{1}\right) \delta_{1}\left(u_{0}, x_{1}\right) v_{0} x_{0}=0
$$

que comparando com (3.42) implica $\delta_{1}\left(u_{0}, x_{1}\right) v_{0}\left[x_{0}, D_{1}\left(y_{1}\right)\right]=0$, para quaisquer $u_{0}, v_{0} \in U_{0}, x_{0} \in$ $\mathcal{A}_{0}$ e $x_{1}, y_{1} \in \mathcal{A}_{1}$. Portanto,

$$
\left.\delta_{1}\left(U_{0}, \mathcal{A}_{1}\right)\left(\left[\mathcal{A}_{0}, D_{1}\left(\mathcal{A}_{1}\right)\right] U_{0} \delta_{1}\left(U_{0}, \mathcal{A}_{1}\right)\right)\left[\mathcal{A}_{0}, D_{1}\left(\mathcal{A}_{1}\right)\right]\right)=0 \text { e }
$$

assim, $\delta_{1}\left(U_{0}, \mathcal{A}_{1}\right)\left[\mathcal{A}_{0}, D_{1}\left(\mathcal{A}_{1}\right)\right]=0$.

Desse modo, pelo Lema 1.2 .11 , segue $\left[\delta_{1}\left(U_{0}, \mathcal{A}_{1}\right), D_{1}\left(\mathcal{A}_{1}\right)\right]=0$, o que implica que valem as seguintes igualdades

$$
\begin{aligned}
{\left[\delta_{1}\left(u_{0}, x_{1}\right) x_{0}, D_{1}\left(y_{1}\right)\right]=} & \delta_{1}\left(u_{0}, x_{1}\right) x_{0} D_{1}\left(y_{1}\right)-D_{1}\left(y_{1}\right) \delta_{1}\left(u_{0}, x_{1}\right) x_{0} \\
= & \delta_{1}\left(u_{0}, x_{1}\right) x_{0} D_{1}\left(y_{1}\right)-\delta_{1}\left(u_{0}, x_{1}\right) D_{1}\left(y_{1}\right) x_{0}+\delta_{1}\left(u_{0}, x_{1}\right) D_{1}\left(y_{1}\right) x_{0} \\
& -D_{1}\left(y_{1}\right) \delta_{1}\left(u_{0}, x_{1}\right) x_{0} \\
= & \delta_{1}\left(u_{0}, x_{1}\right)\left[x_{0}, D_{1}\left(y_{1}\right)\right]+\left[\delta_{1}\left(u_{0}, x_{1}\right), D_{1}\left(y_{1}\right)\right] x_{0}=0,
\end{aligned}
$$

para todos $u_{0} \in U_{0}, x_{0} \in \mathcal{A}_{0}$ e $x_{1}, y_{1} \in \mathcal{A}_{1}$. Portanto, $\delta_{1}\left(U_{0}, \mathcal{A}_{1}\right) U_{0} D_{1}\left(\mathcal{A}_{1}\right)=0$, por $(3.40)$; e, por (3.38), chegamos a

$$
\begin{aligned}
\delta_{1}\left(u_{0}, x_{1}\right) v_{0} \delta_{1}\left(u_{0}, x_{1}\right)= & \delta_{1}\left(u_{0}, x_{1}\right) v_{0}\left(D_{1}\left(u_{0} x_{1}\right)-D_{1}\left(u_{0}\right) x_{1}-u_{0} D_{1}\left(x_{1}\right)\right) \\
= & \delta_{1}\left(u_{0}, x_{1}\right) v_{0} D_{1}\left(u_{0} x_{1}\right)-\left(\delta_{1}\left(u_{0}, x_{1}\right) v_{0} D_{1}\left(u_{0}\right)\right) x_{1} \\
& -\delta_{1}\left(u_{0}, x_{1}\right)\left(v_{0} u_{0}\right) D_{1}\left(x_{1}\right)=0
\end{aligned}
$$

para todo $v_{0} \in U_{0}$.

Assim, temos $\left(\delta_{1}\left(U_{0}, \mathcal{A}_{1}\right) U_{0}\right) U_{0}\left(\delta_{1}\left(U_{0}, \mathcal{A}_{1}\right) U_{0}\right)=\left(U_{0} \delta_{1}\left(U_{0}, \mathcal{A}_{1}\right)\right) U_{0}\left(U_{0} \delta_{1}\left(U_{0}, \mathcal{A}_{1}\right)\right)=0$, de onde segue $\delta_{1}\left(U_{0}, \mathcal{A}_{1}\right) U_{0}=U_{0} \delta_{1}\left(U_{0}, \mathcal{A}_{1}\right)=0$, pois estes conjuntos estão contidos em $U_{0}$ e $U_{0}$ é semiprima. Consequentemente, $\delta_{1}\left(U_{0}, \mathcal{A}_{1}\right) U=U \delta_{1}\left(U_{0}, \mathcal{A}_{1}\right)=0$, por (3.38). 
Agora, por (3.26), obtemos

$$
\begin{aligned}
0 & =\delta_{1}\left(u_{0}, x_{1}\right) x_{0} D_{1}\left(\left(\delta_{1}\left(u_{0}, x_{1}\right) y_{0}\right) v_{0}\right) \\
& =\delta_{1}\left(u_{0}, x_{1}\right)\left(x_{0} D_{1}\left(\delta_{1}\left(u_{0}, x_{1}\right) y_{0}\right) v_{0}\right)+\delta_{1}\left(u_{0}, x_{1}\right) x_{0} \delta_{1}\left(u_{0}, x_{1}\right) y_{0} D_{1}\left(v_{0}\right) \\
& =\delta_{1}\left(u_{0}, x_{1}\right) x_{0} \delta_{1}\left(u_{0}, x_{1}\right) y_{0} D_{1}\left(v_{0}\right)
\end{aligned}
$$

para todos $u_{0}, v_{0} \in U_{0}, x_{0}, y_{0} \in \mathcal{A}_{0}$ e $x_{1} \in \mathcal{A}_{1}$. Portanto,

$$
\left(\delta_{1}\left(u_{0}, x_{1}\right) x_{0} D_{1}\left(v_{0}\right)\right) \mathcal{A}_{1}\left(\delta_{1}\left(u_{0}, x_{1}\right) x_{0} D_{1}\left(v_{0}\right)\right)=0,
$$

pois $x_{0} D_{1}\left(v_{0}\right) \mathcal{A}_{1} \subseteq \mathcal{A}_{0}$, de onde resulta $\delta_{1}\left(U_{0}, \mathcal{A}_{1}\right) \mathcal{A}_{0} D_{1}\left(U_{0}\right)=0$, por (i) do Lema 1.2 .8 , pois $\delta_{1}\left(U_{0}, \mathcal{A}_{1}\right) \mathcal{A}_{0} D_{1}\left(U_{0}\right) \subseteq \mathcal{A}_{1}$. Finalmente, usando esta última igualdade, (3.39) e $\delta_{1}\left(U_{0}, \mathcal{A}_{1}\right) U=0$, segue

$$
\delta_{1}\left(u_{0}, x_{1}\right) x_{0} \delta_{1}\left(u_{0}, x_{1}\right)=\delta_{1}\left(u_{0}, x_{1}\right) x_{0}\left(D_{1}\left(u_{0} x_{1}\right)-D_{1}\left(u_{0}\right) x_{1}-u_{0} D_{1}\left(x_{1}\right)\right)=0 .
$$

Logo,

$$
\delta_{1}\left(U_{0}, \mathcal{A}_{1}\right)=0
$$

pois $\mathcal{A}_{0}$ é semiprima, o que conclui a prova do teorema, pois em $(3.26),(3.33),(3.37)$ e (3.43) provamos as igualdades

$$
\delta_{1}\left(U_{0}, \mathcal{A}_{0}\right)=\delta_{1}\left(U_{0}, \mathcal{A}_{1}\right)=\delta_{1}\left(U_{1}, \mathcal{A}_{0}\right)=\delta_{1}\left(U_{1}, \mathcal{A}_{1}\right)=0 .
$$

Finalmente, estamos em condições de provar o seguinte teorema que estende o resultado da seção anterior.

Teorema 3.2.3. (Fošner, [11]) Seja $\mathcal{A}=\mathcal{A}_{0} \oplus \mathcal{A}_{1}$ uma superálgebra associativa semiprima e $D=D_{0}+D_{1}$ uma superderivação de Jordan. Então, existem ideais graduados $U$ e $V$ de $\mathcal{A}$ tais que $D_{i}(u x)=D_{i}(u) x+(-1)^{i|u|} u D_{i}(x), i=0,1$, para todos $u \in U$ e $x \in \mathcal{A}$, e $\left[v_{0}, x_{0}\right]=0$, para todos $v_{0} \in V_{0}$ e $x_{0} \in \mathcal{A}_{0}$. Além disso, $U \cap V=0$ e $U \oplus V$ é um ideal essencial de $\mathcal{A}$, isto é, a interseção de $U \oplus V$ com qualquer ideal graduado não nulo de $\mathcal{A}$ é não nula. Se $U=0$, então, $\mathcal{A}_{0}$ é comutativa e se $V=0$, então, $D$ é uma superderivação.

Demonstração. Observe que definindo $U$ como sendo o ideal de $\mathcal{A}$ gerado por $\left[\mathcal{A}_{0}, \mathcal{A}_{0}\right]$, então, $U$ é graduado e os Teoremas 3.2.1 e 3.2.2 implicam

$$
\begin{aligned}
D(u x) & =D_{0}(u x)+D_{1}(u x) \\
& =D_{0}(u) x+u D_{0}(x)+D_{1}(u) x+(-1)^{|u|} u D_{1}(x),
\end{aligned}
$$

para todos $u \in U$ e $x \in \mathcal{A}$. Agora, se $V=\operatorname{Ann}(U)=\{v \in \mathcal{A} \mid v u=u v=0$, para todo $u \in U\}$, o anulador de $U$, então, $V$ é também um ideal graduado. Com efeito, considere $v \in V$, queremos provar que $\sigma(v) \in V$. Mas, $\sigma(U)=U$, logo para cada $u \in U, u=\sigma(\bar{u}), \bar{u} \in U$, e segue que

$$
\sigma(v) u=\sigma(v) \sigma(\bar{u})=\sigma(v \bar{u})=\sigma(0)=0,
$$

do mesmo modo, $u \sigma(v)=0$, isto é, $\sigma(v) \in V$. E se $v_{0} \in V_{0}$, temos

$$
\left[v_{0}, x_{0}\right] y_{0}\left[v_{0}, x_{0}\right]=v_{0}\left(x_{0} y_{0}\left[v_{0}, x_{0}\right]\right)-x_{0} v_{0}\left(y_{0}\left[v_{0}, x_{0}\right]\right)=0
$$


para todos $x_{0}, y_{0} \in \mathcal{A}_{0}$, pois $x_{0} y_{0}\left[v_{0}, x_{0}\right], y_{0}\left[v_{0}, x_{0}\right] \in U_{0}$. Logo, $\left[v_{0}, x_{0}\right]=0$, para todos $v_{0} \in V_{0}$ e $x_{0} \in \mathcal{A}_{0}$, pois $\mathcal{A}_{0}$ é semiprima. Agora, vamos mostrar que se verifica

$$
\delta_{1}\left(\mathcal{A}_{0}, \mathcal{A}_{1}\right) \perp\left[\mathcal{A}_{0}, \mathcal{A}_{0}\right] .
$$

Para isso, inicialmente considere o elemento $D_{1}\left(u_{0} x_{0} y_{1}\right)$, onde $u_{0} \in U_{0}, x_{0} \in \mathcal{A}_{0}$ e $y_{1} \in \mathcal{A}_{1}$. Por um lado, temos

$$
D_{1}\left(u_{0}\left(x_{0} y_{1}\right)\right)=D_{1}\left(u_{0}\right) x_{0} y_{1}+u_{0} D_{1}\left(x_{0} y_{1}\right)
$$

e por outro lado, valem

$$
\begin{aligned}
D_{1}\left(\left(u_{0} x_{0}\right) y_{1}\right) & =D_{1}\left(u_{0} x_{0}\right) y_{1}+u_{0} x_{0} D_{1}\left(y_{1}\right) \\
& =D_{1}\left(u_{0}\right) x_{0} y_{1}+u_{0} D_{1}\left(x_{0}\right) y_{1}+u_{0} x_{0} D_{1}\left(y_{1}\right) .
\end{aligned}
$$

Assim, comparando as expressões obtidas, temos $u_{0}\left(D_{1}\left(x_{0} y_{1}\right)-D_{1}\left(x_{0}\right) y_{1}-x_{0} D_{1}\left(y_{1}\right)\right)=0$, isto é, $u_{0} \delta_{1}\left(x_{0}, y_{1}\right)=0$. Em particular, escolhendo $u_{0}=\left[y_{0}, z_{0}\right] w_{0} \in U_{0}$ chegamos a $\left[y_{0}, z_{0}\right] w_{0} \delta_{1}\left(x_{0}, y_{1}\right)=0$, para todos $x_{0}, y_{0}, z_{0}, w_{0} \in \mathcal{A}_{0}$ e $y_{1} \in \mathcal{A}_{1}$, ou seja,

$$
\left[\mathcal{A}_{0}, \mathcal{A}_{0}\right] \mathcal{A}_{0} \delta_{1}\left(\mathcal{A}_{0}, \mathcal{A}_{1}\right)=0
$$

Além disso, note que a igualdade $\mathcal{A}_{1}\left[\mathcal{A}_{0}, \mathcal{A}_{0}\right] \mathcal{A}_{1} \delta_{1}\left(\mathcal{A}_{0}, \mathcal{A}_{1}\right)=0$ é válida, pois $\mathcal{A}_{1}\left[\mathcal{A}_{0}, \mathcal{A}_{0}\right] \mathcal{A}_{1} \subseteq U_{0}$, o que implica

$$
\left(\left[\mathcal{A}_{0}, \mathcal{A}_{0}\right] \mathcal{A}_{1} \delta_{1}\left(\mathcal{A}_{0}, \mathcal{A}_{1}\right)\right) \mathcal{A}_{1}\left(\left[\mathcal{A}_{0}, \mathcal{A}_{0}\right] \mathcal{A}_{1} \delta_{1}\left(\mathcal{A}_{0}, \mathcal{A}_{1}\right)\right)=0
$$

Portanto, por (i) do Lema 1.2.8, concluímos

$$
\left[\mathcal{A}_{0}, \mathcal{A}_{0}\right] \mathcal{A}_{1} \delta_{1}\left(\mathcal{A}_{0}, \mathcal{A}_{1}\right)=0
$$

pois este último conjunto está contido em $\mathcal{A}_{1}$. Assim, por (3.45) e (3.46), temos que (3.44) está provado.

Claramente se $U=0$, então, $\left[\mathcal{A}_{0}, \mathcal{A}_{0}\right]=0$, isto é, $\mathcal{A}_{0}$ é comutativa. Observe que

$$
\delta_{0}(x, y), \delta_{1}(x, y) \in V
$$

para $x, y \in \mathcal{A}_{0} \cup \mathcal{A}_{1}$, por (3.12), (3.13), (3.21), (3.29), (3.44) e pelo Lema 3.2.4. Portanto, $V=0$ implica que $D$ é uma superderivação, ou seja, a soma de superderivações de graus 0 e 1 .

Finalmente, por definição, temos $U V=0$ e, portanto, $U \cap V=0$, pois $(U \cap V)^{2} \subseteq U V=0$ e $\mathcal{A}$ é semiprima. Supondo $(U+V) \cap I=0$, para algum ideal graduado $I$ de $\mathcal{A}$, temos $U I=V I=0$, o que implica $I \subseteq \operatorname{Ann}(U) \cap \operatorname{Ann}(V)=\operatorname{Ann}(U) \cap \operatorname{Ann}(\operatorname{Ann}(U))=0$. De fato, provamos acima que o anulador de um ideal graduado é graduado. Além disso, a interseção de ideais graduados é um ideal graduado. Portanto $\operatorname{Ann}(U) \cap \operatorname{Ann}(\operatorname{Ann}(U))$ é ideal graduado. Considere agora um elemento $x_{i} \in(\operatorname{Ann}(U) \cap \operatorname{Ann}(\operatorname{Ann}(U))) \cap \mathcal{A}_{i}, i=0$ ou 1 . Temos que o ideal graduado de $\mathcal{A}, J=\operatorname{ideal}\left\langle x_{i}\right\rangle$, é tal que $J^{2}=0$, e assim, $J=0$ e $x_{i}=0$. Portanto $\operatorname{Ann}(U) \cap \operatorname{Ann}(\operatorname{Ann}(U))=0$, como queríamos.

Desse modo, $U \oplus V$ é um ideal essencial de $\mathcal{A}$ e a demonstração está completa.

Observemos que esse teorema também mostra que a restrição de $D$ a $U$ é uma superderivação 
e que $V$ vista como superálgebra tem parte par comutativa. Encerramos esse capítulo com um exemplo mostrando que, em geral, $U$ e $V$ não podem ser escolhidos de modo que sua soma seja igual a $\mathcal{A}$.

Exemplo 3.2.1. Sejam $A=A_{0} \oplus A_{1}$ e $B=B_{0} \oplus B_{1}$ superálgebras associativas primas satisfazendo as seguintes condições: nenhuma delas contêm um elemento identidade, $A_{0}$ é uma álgebra não comutativa, $B$ é uma álgebra comutativa e $B_{1} \neq 0$. Por exemplo, pode-se tomar $A$ como sendo a superálgebra trivial de todos os operadores de posto finito de um espaço vetorial de dimensão infinita sobre um corpo $\Phi$ e $B=X \Phi[X]$, a álgebra de polinômios sobre $\Phi$ com termo constante nulo, com a graduação $B_{0}=\Phi\left[X^{2}\right]$ e $B_{1}=X \Phi\left[X^{2}\right]$. Seja $\mathcal{A}=A \oplus B \oplus \Phi$ a álgebra resultante do processo de adjunção de identidade à álgebra $A \oplus B$. Defina $\mathcal{A}_{0}=A_{0} \oplus B_{0} \oplus \Phi 1$ e $\mathcal{A}_{1}=A_{1} \oplus B_{1}$. Desse modo, $\mathcal{A}$ se torna uma superálgebra associativa semiprima cuja parte par é não comutativa. Considere $b=b_{0}+b_{1} \in B$ um elemento tal que $b_{0} \neq 0$ e defina $D: \mathcal{A} \rightarrow \mathcal{A}$ por $D\left(x_{0}+x_{1}+y_{0}+y_{1}+\lambda\right)=b y_{1}$, para quaisquer $x_{0} \in A_{0}, x_{1} \in A_{1}, y_{0} \in B_{0}, y_{1} \in B_{1}$ e $\lambda \in \Phi$. Então, $D$ é uma superderivação de Jordan que não é uma superderivação. Além disso, como $\mathcal{A}$ é uma álgebra com unidade cujos únicos idempotentes centrais são 0 e $1, \mathcal{A}$ não contém ideais próprios $U$ e $V$ tais que $\mathcal{A}=U \oplus V$. 


\section{Capítulo 4}

\section{Homomorfismos e superhomomorfismos de Jordan em anéis e superálgebras primas}

O estudo de aplicações aditivas entre anéis, que preservam quadrados, foi iniciado por Ancochea ([1],[2]), em conexão com problemas que surgiram da geometria projetiva. Kaplansky e Hua ([19],[24]), deram sequência aos estudos de Ancochea, obtendo resultados novos. Em seguida, Jacobson e Rickart ([20]), passaram a estudar mais profundamente essas aplicações.

Nesse capítulo, apresentaremos alguns resultados de Herstein e Smiley ([15],[31]), de Beidar, Bresar e Chebotar [3], a respeito de homomorfismos e superhomomorfismos de Jordan. Na primeira seção, lidaremos com homomorfismos de Jordan sobrejetores de um anel em um anel primo e na segunda provaremos uma generalização para superálgebras.

\subsection{Homomorfismos de Jordan sobre anéis primos}

Iniciamos recordando algumas notações. Dado um anel associativo $(A,+, \cdot)$, denotamos o produto de Jordan de $A$ por $a \circ b=a b+b a$, onde $a, b \in A$ e $a b$ representa o produto de $a$ por $b$; e o anel $(A,+, \circ)$ é chamado de anel de Jordan de $A$. O comutador de quaisquer dois elementos $a$ e $b$ de $A$ é definido como sendo $[a, b]=a b-b a$. Nesta seção, todos os anéis considerados serão associativos.

Uma aplicação $\phi$, de um anel $A$ em um anel $A^{\prime}$, é dita um homomorfismo de Jordan se for aditiva e se $\phi(a \circ b)=\phi(a) \circ \phi(b)$, para quaisquer $a, b \in A$. Em outras palavras, $\phi$ é um homomorfismo de Jordan se

(i) $\phi(a+b)=\phi(a)+\phi(b)$;

(ii) $\phi(a b+b a)=\phi(a) \phi(b)+\phi(b) \phi(a)$,

para todos $a, b \in A$. Observe que se a característica de $A^{\prime}$ é diferente de 2 , esta definição se torna equivalente a $\phi$ ser aditiva e $\phi\left(a^{2}\right)=\phi(a)^{2}$, para todo $a \in A$. De fato, se assumimos a primeira definição, então, escolhendo $b=a$, temos $2 \phi\left(a^{2}\right)=2 \phi(a)^{2}$. Portanto, vale a segunda definição. Reciprocamente, se a segunda definição é assumida, então, linearizando a igualdade $\phi\left(a^{2}\right)=\phi(a)^{2}$, 
obtemos $\phi(a b+b a)=\phi(a) \phi(b)+\phi(b) \phi(a)$ e a primeira definição é válida. Além disso, também temos o seguinte

Lema 4.1.1. Se $\phi: A \rightarrow A^{\prime}$ é um homomorfismo de Jordan, então, as seguintes igualdades são válidas:

(i) $\phi(a b a)=\phi(a) \phi(b) \phi(a)$, para quaisquer $a, b \in A$;

(ii) $\phi(a b c+c b a)=\phi(a) \phi(b) \phi(c)+\phi(c) \phi(b) \phi(a)$, para quaisquer $a, b, c \in A$.

Demonstração. Primeiramente, observe que como $\phi$ é um homomorfismo de Jordan, temos

$$
\begin{aligned}
\phi((a \circ b) \circ a) & =\phi(a \circ b) \circ \phi(a) \\
\phi\left(a b a+b a^{2}+a^{2} b+a b a\right) & =\phi(a) \phi(b) \phi(a)+\phi(b) \phi(a)^{2}+\phi(a)^{2} \phi(b)+\phi(a) \phi(b) \phi(a) \\
2 \phi(a b a)+\phi\left(b \circ a^{2}\right) & =2 \phi(a) \phi(b) \phi(a)+\phi(b) \circ \phi\left(a^{2}\right) \\
\phi(a b a) & =\phi(a) \phi(b) \phi(a),
\end{aligned}
$$

para todos $a, b \in A$, o que prova (i). O item (ii) apenas é a linearização da igualdade de (i).

A partir de agora, usaremos as notações $a^{b}=\phi(a b)-\phi(a) \phi(b)$ e $a_{b}=\phi(a b)-\phi(b) \phi(a)$, onde $a, b \in A$. Seja $\phi: A \rightarrow A^{\prime}$ um homomorfismo de Jordan. Observe que vale $a^{b}=0$, para todos $a, b \in A$, se, e somente se, $\phi$ é um homomorfismo; e $a_{b}=0$, para todos $a, b \in A$, se, e somente se, $\phi$ é um antihomomorfismo, isto é, uma aplicação aditiva tal que $\phi(a b)=\phi(b) \phi(a)$, para todos $a, b \in A$. Fixadas estas notações, efetuaremos alguns cálculos.

Lema 4.1.2. Se $\phi: A \rightarrow A^{\prime}$ é um homomorfismo de Jordan e $a, b \in A$ são elementos arbitrários, então, são válidas as seguintes propriedades:

(1) $b^{a}=-a^{b}$ e $b_{a}=-a_{b}$

(2) $[\phi(a), \phi(b)]=a_{b}-a^{b}$;

(3) $\phi([a, b])=2 \phi(a b)-\phi(a \circ b)=a_{b}+a^{b}$;

(4) $a^{b} a_{b}=a_{b} a^{b}=0$.

Demonstração. (1)

$$
\begin{aligned}
b^{a}+a^{b} & =\phi(b a)-\phi(b) \phi(a)+\phi(a b)-\phi(a) \phi(b) \\
& =\phi(b a+a b)-(\phi(b) \phi(a)+\phi(a) \phi(b))=0 \mathrm{e} \\
b_{a}+a_{b} & =\phi(b a)-\phi(a) \phi(b)+\phi(a b)-\phi(b) \phi(a) \\
& =\phi(b a+a b)-(\phi(b) \phi(a)+\phi(a) \phi(b))=0 .
\end{aligned}
$$

(2)

$$
\begin{aligned}
{[\phi(a), \phi(b)] } & =\phi(a) \phi(b)-\phi(b) \phi(a) \\
& =\phi(a b)-\phi(b) \phi(a)-\phi(a b)+\phi(a) \phi(b) \\
& =a_{b}-a^{b} .
\end{aligned}
$$


$(3)$

$$
\begin{aligned}
\phi([a, b]) & =\phi(a b)-\phi(b a) \\
& =\phi(a b)-\phi(b) \phi(a)-\phi(b a)+\phi(b) \phi(a) \\
& =a_{b}-b^{a}=a_{b}+a^{b} .
\end{aligned}
$$

(4) É uma consequência do Lema 4.1.1. De fato,

$$
\begin{aligned}
a^{b} a_{b} & =(\phi(a b)-\phi(a) \phi(b))(\phi(a b)-\phi(b) \phi(a)) \\
& =\phi(a b)^{2}+\phi(a) \phi(b)^{2} \phi(a)-(\phi(a b) \phi(b) \phi(a)+\phi(a) \phi(b) \phi(a b)) \mathrm{e} \\
& =\phi\left((a b)^{2}+a b^{2} a-(a b) b a+a b(a b)\right)=\phi(0)=0 \\
a_{b} a^{b} & =(\phi(a b)-\phi(b) \phi(a))(\phi(a b)-\phi(a) \phi(b)) \\
& =\phi(a b)^{2}+\phi(b) \phi(a)^{2} \phi(b)-(\phi(a b) \phi(a) \phi(b)+\phi(b) \phi(a) \phi(a b)) \\
& =\phi\left((a b)^{2}+b a^{2} b-(a b) a b-(b a) a b\right)=\phi(0)=0 . \quad \square
\end{aligned}
$$

Além disso, pelo Lema 4.1.1, também temos

$$
\begin{aligned}
\phi(r) a^{b}+a_{b} \phi(r) & =\phi(r) \phi(a b)-\phi(r) \phi(a) \phi(b)+\phi(a b) \phi(r)-\phi(b) \phi(a) \phi(r) \\
& =\phi(r) \circ \phi(a b)-(\phi(r) \phi(a) \phi(b)+\phi(b) \phi(a) \phi(r)) \\
& =\phi(r \circ(a b))-\phi(r a b+b a r) \\
& =\phi(r a b+a b r-r a b-b a r)=\phi(a b r-b a r) \\
& =\phi([a, b] r),
\end{aligned}
$$

para todos $a, b, r \in A$, isto é,

$$
\phi(r) a^{b}+a_{b} \phi(r)=\phi([a, b] r) \text {, para quaisquer } a, b, r \in A \text {. }
$$

Analogamente,

$$
\begin{aligned}
a^{b} \phi(r)+\phi(r) a_{b} & =\phi(a b) \phi(r)-\phi(a) \phi(b) \phi(r)+\phi(r) \phi(a b)-\phi(r) \phi(b) \phi(a) \\
& =\phi(a b) \circ \phi(r)-(\phi(a) \phi(b) \phi(r)+\phi(r) \phi(b) \phi(a)) \\
& =\phi((a b) \circ r)-\phi(a b r+r b a) \\
& =\phi(a b r+r a b-a b r-r b a)=\phi(r a b-r b a) \\
& =\phi(r[a, b]),
\end{aligned}
$$

para quaisquer $a, b, r \in A$, ou seja,

$$
a^{b} \phi(r)+\phi(r) a_{b}=\phi(r[a, b]), \text { para todos } a, b, r \in A
$$

Assim, multiplicando (4.1) à esquerda por $a^{b}$, por (4) do Lema 4.1.1, obtemos

$$
a^{b} \phi(r) a^{b}=a^{b} \phi([a, b] r), \text { para quaisquer } a, b, r \in A .
$$


Do mesmo modo, multiplicando (4.1) à direita por $a_{b}$, segue

$$
a_{b} \phi(r) a_{b}=\phi([a, b] r) a_{b}, \text { para todos } a, b, r \in A .
$$

Portanto, tomando $r=[a, b] r$ em (4.2), por (4.3), (4.4), (i) do Lema 4.1.1 e (3) do Lema 4.1.2, temos

$$
\begin{aligned}
a^{b} \phi([a, b] r)+\phi([a, b] r) a_{b} & =\phi([a, b] r[a, b]) \\
a^{b} \phi(r) a^{b}+a_{b} \phi(r) a_{b} & =\phi([a, b]) \phi(r) \phi([a, b]) \\
a^{b} \phi(r) a^{b}+a_{b} \phi(r) a_{b} & =\left(a_{b}+a^{b}\right) \phi(r)\left(a_{b}+a^{b}\right),
\end{aligned}
$$

isto é,

$$
a_{b} \phi(r) a^{b}+a^{b} \phi(r) a_{b}=0 \text {, para quaisquer } a, b, r \in A \text {. }
$$

Agora, podemos provar a seguinte proposição.

Proposição 4.1.1. Seja $\phi: A \rightarrow A^{\prime}$ um homomorfismo de Jordan sobrejetor de um anel $A$ em um anel primo $A^{\prime}$. Se a característica de $A^{\prime}$ é diferente de 2 , então, uma das três afirmações é válida.

(i) $x^{y}=0$, para todos $x, y \in A$;

(ii) $x_{y}=0$, para todos $x, y \in A$;

(iii) $\left(x_{y}\right)^{2}=\left(x^{y}\right)^{2}=0$, para todos $x, y \in A$.

Demonstraçẫo. Vamos provar que se (iii) não é verdadeira, então, (i) ou (ii) é válida. Suponha que existam $c, d \in A$ tais que $\left(c^{d}\right)^{2} \neq 0$. Escolhendo $a=c$ e $b=d$ em (4.5), segue $c_{d} \phi(r) c^{d}+c^{d} \phi(r) c_{d}=0$, que multiplicando à esquerda por $c^{d}$ resulta em $\left(c^{d}\right)^{2} \phi(r) c_{d}=0$, para todo $r \in A$, pois $c^{d} c_{d}=0$, o que implica $\left(c^{d}\right)^{2} A^{\prime} c_{d}=0$, pela sobrejetividade de $\phi$. Logo, como $A^{\prime}$ é um anel primo e $\left(c^{d}\right)^{2} \neq 0$, concluímos $c_{d}=0$.

Além disso, linearizando (4.5) e $a^{b} a_{b}=0$ em $b$, obtemos

$$
\begin{gathered}
a_{b} \phi(r) a^{x}+a_{x} \phi(r) a^{b}+a^{b} \phi(r) a_{x}+a^{x} \phi(r) a_{b}=0 \mathrm{e} \\
a^{b} a_{x}+a^{x} a_{b}=0,
\end{gathered}
$$

para todos $a, b, r, x \in A$. Assim, novamente tomando $a=c$ e $b=d$, seguem $c_{x} \phi(r) c^{d}+c^{d} \phi(r) c_{x}=0$ e $c^{d} c_{x}=0$, para quaisquer $r, x \in A$, pois $c_{d}=0$. Consequentemente, multiplicando a penúltima igualdade à esquerda por $c^{d}$, como $c^{d} c_{x}=0$, temos $\left(c^{d}\right)^{2} \phi(r) c_{x}=0$, para todo $r \in A$, o que implica $c_{x}=0$ e $x_{c}=-c_{x}=0$, para todo $x \in A$, pois $\phi$ é sobrejetor, $A^{\prime}$ é anel primo e $\left(c^{d}\right)^{2} \neq 0$. Analogamente, como $\left(d^{c}\right)^{2}=\left(c^{d}\right)^{2} \neq 0$, pelos mesmos cálculos anteriores, podemos concluir $d_{x}=$ $x_{d}=0$, para todo $x \in A$.

Agora, a linearização de (4.6) resulta em

$$
\begin{aligned}
a_{b} \phi(r) y^{x}+y_{b} \phi(r) a^{x}+a_{x} \phi(r) y^{b}+y_{x} \phi(r) a^{b}+a^{b} \phi(r) y_{x}+y^{b} \phi(r) a_{x} \\
+a^{x} \phi(r) y_{b}+y^{x} \phi(r) a_{b}=0,
\end{aligned}
$$

para todos $a, b, r, y, x \in A$. Desse modo, escolhendo $a=c$ e $b=d$, obtemos

$$
c^{d} \phi(r) y_{x}+y_{x} \phi(r) c^{d}=0,
$$


para quaisquer $r, y, x \in A$, pois $c_{d}=y_{d}=c_{x}=0$

Prosseguindo, linearizando (4.7), chegamos a

$$
a^{b} y_{x}+y^{b} a_{x}+a^{x} y_{b}+y^{x} a_{b}=0
$$

para todos $a, b, r, x, y \in A$. Assim, para $a=c$ e $b=d$, temos $c^{d} y_{x}=0$, para todos $x, y \in A$, pois $c_{x}=y_{d}=c_{d}=0$. Consequentemente, multiplicando (4.8) à esquerda por $c^{d}$, segue $\left(c^{d}\right)^{2} \phi(r) y_{x}=0$, para todo $r \in A$, o que implica $y_{x}=0$, para todos $x, y \in A$, pois $\phi$ é sobrejetor, $A^{\prime}$ é primo e $\left(c^{d}\right)^{2} \neq 0$. Logo, (ii) é válida.

Suponhamos que existam $c, d \in A$ tais que $\left(c_{d}\right)^{2} \neq 0$. Escolhendo $a=c$ e $b=d$ em (4.5), segue $c_{d} \phi(r) c^{d}+c^{d} \phi(r) c_{d}=0$. Assim, multiplicando esta igualdade à direita por $c_{d}$, temos $c^{d} \phi(r)\left(c_{d}\right)^{2}=0$, para todo $r \in A$, pois $c^{d} c_{d}=0$, o que implica $c^{d} A^{\prime}\left(c_{d}\right)^{2}=0$, de onde é possível concluir $c^{d}=0$.

Além disso, (4.6) e (4.7) no caso particular $a=c$ e $b=d$ nos fornecem $c_{d} \phi(r) c^{x}+c^{x} \phi(r) c_{d}=0$ e $c^{x} c_{d}=0$, para todos $r, x \in A$, pois $c^{d}=0$. Desse modo, multiplicando a penúltima igualdade à direita por $c_{d}$, obtemos $c^{x} \phi(r)\left(c_{d}\right)^{2}=0$, para todo $r \in A$, pois $c^{x} c_{d}=0$, o que implica $c^{x}=0$, e também $x^{c}=-c_{x}=0$, para todo $x \in A$, novamente pois $\phi$ é sobrejetor, $A^{\prime}$ é um anel primo e $\left(c_{d}\right)^{2} \neq 0$. De modo análogo, como $\left(d_{c}\right)^{2}=\left(c_{d}\right)^{2} \neq 0$, podemos concluir $d^{x}=x^{d}=0$, para todo $x \in A$.

Finalmente, linearizando (4.6), escolhendo $a=c, b=d$ e usando $c^{d}=c^{x}=y^{d}=0$, obtemos

$$
y^{x} \phi(r) c_{d}+c_{d} \phi(r) y^{x}=0
$$

para todos $r, y, x \in A$; e linearizando (4.7), tomando $a=c, b=d$, como as igualdades $c^{x}=y^{d}=$ $c^{d}=0$ são válidas, temos $y^{x} c_{d}=0$, para quaisquer $x, y \in A$. Consequentemente, multiplicando (4.9) à direita por $c_{d}$, segue $y^{x} \phi(r)\left(c_{d}\right)^{2}=0$, para todo $r \in A$. Logo, $y^{x}=0$, para todos $x, y \in A$, o que prova (i).

Antes de apresentarmos o resultado principal dessa seção, observemos que se ocorrer a terceira possibilidade da Proposição 4.1.1, isto é, $\left(x_{y}\right)^{2}=\left(x^{y}\right)^{2}=0$, para todos $x, y \in A$, então, pelas propriedades (2) e (4) do Lema 4.1.2, temos

$$
\begin{aligned}
{[\phi(x), \phi(y)]^{2} } & =\left(x_{y}-x^{y}\right)^{2} \\
& =\left(x_{y}\right)^{2}-x_{y} x^{y}-x^{y} x_{y}+\left(x^{y}\right)^{2}=0
\end{aligned}
$$

para todos $x, y \in A$, o que significa que $A^{\prime}$ é um anel primo tal que $[a, b]^{2}=0$, para quaisquer $a, b \in A^{\prime}$, pois $\phi$ é sobrejetor. Assim, pelo Lema 1.1.1, $A^{\prime}$ é um anel comutativo e não possui divisores de zero não nulos. Finalmente, podemos concluir o seguinte

Teorema 4.1.1. (Smiley, [31]) Seja $\phi: A \rightarrow A^{\prime}$ um homomorfismo de Jordan sobrejetor de um anel $A$ em um anel primo $A^{\prime}$. Se a característica de $A^{\prime}$ é diferente de 2, então, $\phi$ é um homomorfismo ou $\phi$ é um antihomomorfismo.

Demonstração. Pela Proposição 4.1.1, sabemos que $\phi$ é um homomorfismo ou $\phi$ é um antihomomorfismo ou $\left(x_{y}\right)^{2}=\left(x^{y}\right)^{2}=0$, para todos $x, y \in A$. Entretanto, se ocorrer este último caso, pelo que foi observado anteriormente, temos que $A^{\prime}$ é um anel comutativo que não possui divisores de zero não nulos, e portanto, $\phi$ é homomorfismo e antihomomorfismo. 
Além disso, observamos que a condição de que a característica de $A^{\prime}$ seja diferente de 2 é usada somente nas igualdades $\phi\left(a^{2}\right)=\phi(a)^{2}$ e $\phi(a b a)=\phi(a) \phi(b) \phi(a)$. Desse modo, assumindo que estas duas igualdades são válidas, podemos omitir a condição citada.

\subsection{Superhomomorfismos de Jordan sobre superálgebras primas}

Nessa seção, usaremos alguns resultados do Capítulo 1, a saber o Lema 1.2.4, que afirma que se $\mathcal{A}=\mathcal{A}_{0} \oplus \mathcal{A}_{1}$ é uma superálgebra associativa prima, então, $\mathcal{A}$ e $\mathcal{A}_{0}$ são álgebras semiprimas e pelo menos uma delas é prima; e (2) do Lema 1.2.5, que nos diz que se $\mathcal{A}=\mathcal{A}_{0} \oplus \mathcal{A}_{1}$ é uma superálgebra associativa prima e $a_{0} \in \mathcal{A}_{0}$ e $a_{1} \in \mathcal{A}_{1}$ são elementos homogêneos tais que $a_{0} \mathcal{A}_{1} a_{1}=a_{1} \mathcal{A}_{1} a_{0}=0$, então, $a_{0}=0$ ou $a_{1}=0$. Além disso, recorde que uma superálgebra associativa $\mathcal{A}$ é prima se, e somente se, para quaisquer elementos $a, b \in \mathcal{A}_{0} \cup \mathcal{A}_{1}$ tais que $a \mathcal{A} b=0$, tem-se $a=0$ ou $b=0$; e para uma superálgebra arbitrária $\mathcal{A}$, introduzindo um novo produto dado por

$$
x \circ_{s} y=\frac{1}{2}\left(x y+(-1)^{|x||y|} y x\right),
$$

para cada par de elementos $x, y \in \mathcal{A}_{0} \cup \mathcal{A}_{1}, \mathcal{A}$ se torna uma superálgebra de Jordan. Note que se $|x|=0$ ou $|y|=0$, então, $x \circ_{s} y=x \circ y$, onde $\circ$ é o "produto de Jordan" da álgebra $\mathcal{A}$ dado por $x \circ y=\frac{1}{2}(x y+y x)$, e se $|x|=|y|=1$, então, $x \circ_{s} y=\frac{1}{2}[x, y]$.

Definição 4.2.1. Sejam $\mathcal{A}=\mathcal{A}_{0} \oplus \mathcal{A}_{1}$ e $\mathcal{B}=\mathcal{B}_{0} \oplus \mathcal{B}_{1}$ superálgebras. Uma aplicação $\varphi: \mathcal{B} \rightarrow \mathcal{A}$, é dita um superhomomorfismo (respectivamente, um superantihomomorfismo) se $\varphi$ é um homomorfismo de $\Phi$-módulos tal que $\varphi\left(\mathcal{B}_{i}\right) \subseteq \mathcal{A}_{i}, i=0,1$, isto é, $\varphi$ preserva a $\mathbb{Z}_{2}$-graduação, e $\varphi(x y)=\varphi(x) \varphi(y)$ (respectivamente, $\varphi(x y)=(-1)^{|x||y|} \varphi(y) \varphi(x)$ ), para quaisquer $x, y \in \mathcal{B}_{0} \cup \mathcal{B}_{1}$.

Definição 4.2.2. Sejam $\mathcal{A}=\mathcal{A}_{0} \oplus \mathcal{A}_{1}$ e $\mathcal{B}=\mathcal{B}_{0} \oplus \mathcal{B}_{1}$ superálgebras. Uma aplicação $\varphi: \mathcal{B} \rightarrow \mathcal{A}$ é chamada de superhomomorfismo de Jordan se $\varphi$ é um homomorfismo de $\Phi$-módulos que

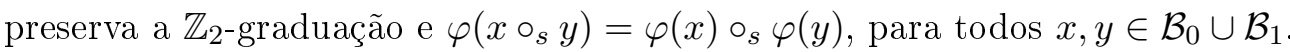

Afirmamos que se $\varphi: \mathcal{B} \rightarrow \mathcal{A}$ é um superhomomorfismo, então, $\varphi$ é um superhomomorfismo de Jordan. De fato, assumindo que $\varphi$ é um superhomomorfismo, temos que $\varphi$ é um homomorfismo de $\Phi$-módulos que preserva a $\mathbb{Z}_{2}$-graduação e tal que

$$
\begin{aligned}
\varphi\left(x \circ_{s} y\right) & =\varphi(x y)+(-1)^{|x||y|} \varphi(y x) \\
& =\varphi(x) \varphi(y)+(-1)^{|\varphi(x)||\varphi(y)|} \varphi(y) \varphi(x) \\
& =\varphi(x) \circ_{s} \varphi(y),
\end{aligned}
$$

para todos $x, y \in \mathcal{B}_{0} \cup \mathcal{B}_{1}$. Um resultado análogo também é válido para superantihomomorfismos, pois

$$
\begin{aligned}
\varphi\left(x \circ_{s} y\right) & =\varphi(x y)+(-1)^{|x||y|} \varphi(y x) \\
& =(-1)^{|x||y|} \varphi(y) \varphi(x)+(-1)^{2|x||y|} \varphi(x) \varphi(y) \\
& =\varphi(x) \varphi(y)+(-1)^{|\varphi(x)||\varphi(y)|} \varphi(y) \varphi(x) \\
& =\varphi(x) \circ_{s} \varphi(y),
\end{aligned}
$$

para quaisquer $x, y \in \mathcal{B}_{0} \cup \mathcal{B}_{1}$. 
A seguir, providenciamos alguns exemplos.

Exemplo 4.2.1. Em geral, um superantihomomorfismo entre superálgebras não é um antihomomorfismo de álgebras, contudo o seguinte é válido: se $\Phi$ contém um elemento $i$ tal que $i^{2}=-1 \mathrm{e}$ definimos $l: \mathcal{A} \rightarrow \mathcal{A}$ por $l\left(x_{0}+x_{1}\right)=x_{0}+i x_{1}, x_{i} \in \mathcal{A}_{i}$, então, um homomorfismo de $\Phi$-módulos que preserva a $\mathbb{Z}_{2}$-graduação $\varphi: \mathcal{B} \rightarrow \mathcal{A}$ é um superantihomomorfismo de superálgebras se, e somente se, $l \circ \varphi$ é um antihomomorfismo de álgebras. Com efeito, se $\varphi$ é um superantihomomorfismo, então,

$$
\varphi(x y)=(-1)^{|x||y|} \varphi(y) \varphi(x)
$$

para $x, y \in \mathcal{B}_{0} \cup \mathcal{B}_{1}$. Assim, dados $u=u_{0}+u_{1}$ e $v=v_{0}+v_{1} \in \mathcal{B}$, temos

$$
\begin{aligned}
& (l \circ \varphi)(u v)=l\left(\varphi\left(u_{0} v_{0}\right)+\varphi\left(u_{1} v_{1}\right)+\varphi\left(u_{0} v_{1}\right)+\varphi\left(u_{1} v_{0}\right)\right) \\
& =l\left(\left(\varphi\left(v_{0}\right) \varphi\left(u_{0}\right)-\varphi\left(v_{1}\right) \varphi\left(u_{1}\right)\right)+\left(\varphi\left(v_{1}\right) \varphi\left(u_{0}\right)+\varphi\left(v_{0}\right) \varphi\left(u_{1}\right)\right)\right) \\
& =\left(\varphi\left(v_{0}\right) \varphi\left(u_{0}\right)-\varphi\left(v_{1}\right) \varphi\left(u_{1}\right)\right)+i\left(\varphi\left(v_{1}\right) \varphi\left(u_{0}\right)+\varphi\left(v_{0}\right) \varphi\left(u_{1}\right)\right) \mathrm{e} \\
& (l \circ \varphi)(v) \cdot(l \circ \varphi)(u)=l\left(\varphi\left(v_{0}\right)+\varphi\left(v_{1}\right)\right) \cdot l\left(\varphi\left(u_{0}\right)+\varphi\left(u_{1}\right)\right) \\
& =\left(\varphi\left(v_{0}\right)+i \varphi\left(v_{1}\right)\right)\left(\varphi\left(u_{0}\right)+i \varphi\left(u_{1}\right)\right) \\
& =\varphi\left(v_{0}\right) \varphi\left(u_{0}\right)-\varphi\left(v_{1}\right) \varphi\left(u_{1}\right)+i \varphi\left(v_{1}\right) \varphi\left(u_{0}\right)+i \varphi\left(v_{0}\right) \varphi\left(u_{1}\right) .
\end{aligned}
$$

Portanto, segue $(l \circ \varphi)(u v)=(l \circ \varphi)(v) \cdot(l \circ \varphi)(u)$. Reciprocamente, suponha que $l \circ \varphi$ é um antihomomorfismo e que $\varphi$ é um homomorfismo de $\Phi$-módulos que preserva a $\mathbb{Z}_{2}$-graduação. Dados $x, y \in \mathcal{B}_{0} \cup \mathcal{B}_{1}$, queremos provar que a igualdade $\varphi(x y)=(-1)^{|x||y|} \varphi(y) \varphi(x)$ é válida. Vamos dividir a prova em quatro casos:

(1) Se $x, y \in \mathcal{B}_{0}$, então, $\varphi(x), \varphi(y), \varphi(x y) \in \mathcal{A}_{0} \mathrm{e}$

$$
\begin{aligned}
\varphi(x y) & =l(\varphi(x y)) \\
& =(l \circ \varphi)(y)(l \circ \varphi)(x)=\varphi(y) \varphi(x) ;
\end{aligned}
$$

(2) Se $x \in \mathcal{B}_{0}$ e $y \in \mathcal{B}_{1}$, então, $\varphi(x) \in \mathcal{A}_{0}, \varphi(y), \varphi(x y) \in \mathcal{A}_{1}$ e

$$
\begin{aligned}
\varphi(x y) & =-i l(\varphi(x y)) \\
& =-i(l \circ \varphi)(y)(l \circ \varphi)(x) \\
& =-i^{2} \varphi(y) \varphi(x)=\varphi(y) \varphi(x)
\end{aligned}
$$

(3) Se $x \in \mathcal{B}_{1}$ e $y \in \mathcal{B}_{0}$, então, $\varphi(x), \varphi(x y) \in \mathcal{A}_{1}, \varphi(y) \in \mathcal{A}_{0}$ e

$$
\begin{aligned}
\varphi(x y) & =-i l(\varphi(x y)) \\
& =-i(l \circ \varphi)(y)(l \circ \varphi)(x) \\
& =-i^{2} \varphi(y) \varphi(x)=\varphi(y) \varphi(x)
\end{aligned}
$$

(4) Se $x, y \in \mathcal{B}_{1}$, então, $\varphi(x), \varphi(y) \in \mathcal{A}_{1}, \varphi(x y) \in \mathcal{A}_{0}$ e

$$
\begin{aligned}
\varphi(x y) & =l(\varphi(x y)) \\
& =(l \circ \varphi)(y)(l \circ \varphi)(x) \\
& =i \varphi(y) i \varphi(x)=-\varphi(y) \varphi(x)
\end{aligned}
$$


Logo, $\varphi$ é um superantihomomorfismo de superálgebras.

Exemplo 4.2.2. Seja $\psi: M_{r} \rightarrow M_{r}$ um antihomomorfismo, onde $M_{r}$ é a álgebra das matrizes $r \times r$. Defina $\varphi: M(r) \rightarrow M(r)$ por

$$
\varphi\left(\left[\begin{array}{ll}
A & B \\
C & D
\end{array}\right]\right)=\left[\begin{array}{cc}
\psi(D) & -\psi(B) \\
\psi(C) & \psi(A)
\end{array}\right]
$$

onde $M(r)$ é a superálgebra das matrizes $2 r \times 2 r$ com $\mathbb{Z}_{2}$-graduação dada por

$$
M(r)_{0}=\left\{\left[\begin{array}{cc}
A & 0 \\
0 & D
\end{array}\right] ; A, D \in M_{r}\right\} \text { e } M(r)_{1}=\left\{\left[\begin{array}{cc}
0 & B \\
C & 0
\end{array}\right] ; B, C \in M_{r}\right\} .
$$

Desse modo, $\varphi$ é homomorfismo de $\Phi$-módulos e

$$
\begin{gathered}
\varphi\left(\left[\begin{array}{ll}
A & 0 \\
0 & D
\end{array}\right]\right)=\left[\begin{array}{cc}
\psi(D) & 0 \\
0 & \psi(A)
\end{array}\right] \in M(r)_{0} \mathrm{e} \\
\varphi\left(\left[\begin{array}{ll}
0 & B \\
C & 0
\end{array}\right]\right)=\left[\begin{array}{cc}
0 & -\psi(B) \\
\psi(C) & 0
\end{array}\right] \in M(r)_{1},
\end{gathered}
$$

implicam que $\varphi$ é graduada. Além disso, por exemplo, para $\left[\begin{array}{cc}0 & A \\ B & 0\end{array}\right],\left[\begin{array}{cc}0 & C \\ D & 0\end{array}\right] \in M(r)_{1}$, temos

$$
\begin{aligned}
\varphi\left(\left[\begin{array}{cc}
0 & A \\
B & 0
\end{array}\right]\left[\begin{array}{cc}
0 & C \\
D & 0
\end{array}\right]\right) & =\varphi\left(\left[\begin{array}{cc}
A D & 0 \\
0 & B C
\end{array}\right]\right)=\left[\begin{array}{cc}
\psi(C) \psi(B) & 0 \\
0 & \psi(D) \psi(A)
\end{array}\right] \\
& =-\left[\begin{array}{cc}
0 & -\psi(C) \\
\psi(D) & 0
\end{array}\right]\left[\begin{array}{cc}
0 & -\psi(A) \\
\psi(B) & 0
\end{array}\right] \\
& =(-1)^{1 \cdot 1} \varphi\left(\left[\begin{array}{cc}
0 & C \\
D & 0
\end{array}\right]\right) \varphi\left(\left[\begin{array}{cc}
0 & A \\
B & 0
\end{array}\right]\right)
\end{aligned}
$$

Fazendo cálculos semelhantes, podemos concluir que $\varphi$ é um superantihomomorfismo.

Exemplo 4.2.3. Seja $\mathcal{A}=\mathcal{A}_{0} \oplus \mathcal{A}_{1}$ uma superálgebra que é comutativa como álgebra. Escolha $a_{0} \in \mathcal{A}_{0}$ e defina $\varphi: \mathcal{A} \rightarrow \mathcal{A}$ por $\varphi\left(x_{0}+x_{1}\right)=x_{0}+a_{0} x_{1}$, para todo $x_{0}+x_{1} \in \mathcal{A}$. Então, $\varphi$ é um superhomomorfismo de Jordan que não necessariamente é um superhomomorfismo nem um superantihomomorfismo. De fato, claramente $\varphi$ é um homomorfismo de $\Phi$-módulos que é graduado. Agora, como $\mathcal{A}$ é álgebra comutativa, se $x_{0} \in \mathcal{A}_{0}$, então, $x_{0} \circ_{s} y=y \circ_{s} x_{0}=x_{0} y$, para todo $y \in \mathcal{A} ;$ e se $x_{1}, y_{1} \in \mathcal{A}_{1}$, então, $x_{1} \circ_{s} y_{1}=0$. Assim, seguem as igualdades $\varphi\left(x \circ_{s} y\right)=\varphi\left(x_{0} y_{0}+x_{0} y_{1}+x_{1} y_{0}\right)=x_{0} y_{0}+a_{0} x_{0} y_{1}+a_{0} x_{1} y_{0}=\left(x_{0}+a_{0} x_{1}\right) \circ_{s}\left(y_{0}+a_{0} y_{1}\right)=\varphi(x) \circ_{s} \varphi(y)$.

Por outro lado, para $x_{1}, y_{1} \in \mathcal{A}_{1}$, temos $\varphi\left(x_{1} y_{1}\right)=a_{0} x_{1} y_{1}$, enquanto $\varphi\left(x_{1}\right) \varphi\left(y_{1}\right)=a_{0}^{2} x_{1} y_{1} \mathrm{e}$ $-\varphi\left(y_{1}\right) \varphi\left(x_{1}\right)=-a_{0}^{2} x_{1} y_{1}$.

Exemplo 4.2.4. Sejam $\gamma \in \Phi$ um elemento invertível tal que $\gamma \neq \gamma^{-1}$, e $\mathcal{Q}(\alpha, \beta)$ a superálgebra 
dos quatérnios definida no Exemplo 2.2.2. Se $\varphi: \mathcal{Q}(\alpha, \beta) \rightarrow \mathcal{Q}(\alpha, \beta)$ é tal que

$$
\varphi\left(\lambda_{0}+\lambda_{1} u v+\lambda_{2} u+\lambda_{3} v\right)=\lambda_{0}+\lambda_{1} u v+\lambda_{2} \gamma u+\lambda_{3} \gamma^{-1} v
$$

para todo $\lambda_{0}+\lambda_{1} u v+\lambda_{2} u+\lambda_{3} v \in \mathcal{Q}(\alpha, \beta)$, então, $\varphi$ é um superhomomorfismo de Jordan que não é um superhomomorfismo nem um superantihomomorfismo, pois $\varphi(u u)=\varphi(\alpha)=\alpha$, mas $\varphi(u) \varphi(u)=\gamma^{2} \alpha$.

A partir de agora, para uma aplicação entre (super)álgebras $\varphi: \mathcal{B} \rightarrow \mathcal{A}$, denotamos $\tau(x, y)=$ $\varphi(x y)-\varphi(x) \varphi(y), \omega(x, y)=\varphi(x y)-\varphi(y) \varphi(x)$ e $\rho(x, y)=\varphi(x y)+\varphi(y) \varphi(x)$, para todos $x, y \in \mathcal{B}$.

Proposição 4.2.1. Seja $\varphi$ um homomorfismo sobrejetor de $\Phi$-módulos de uma álgebra $\mathcal{B}$ em uma álgebra $\mathcal{A}$. Suponha que existam subálgebras $\mathcal{B}_{0}$ de $\mathcal{B}$ e $\mathcal{A}_{0}$ de $\mathcal{A}$ tais que as seguintes condições são satisfeitas:

(i) $\varphi\left(x \circ x_{0}\right)=\varphi(x) \circ \varphi\left(x_{0}\right)$, para todos $x \in \mathcal{B}$ e $x_{0} \in \mathcal{B}_{0}$;

(ii) $\varphi\left(\mathcal{B}_{0}\right) \subseteq \mathcal{A}_{0}$;

(iii) $a_{0} \mathcal{A} b_{0} \mathcal{A} a_{0}=0$, onde $a_{0}, b_{0} \in \mathcal{A}_{0}$, implica $a_{0}=0$ ou $b_{0}=0$.

Então, $\left.\varphi\right|_{\mathcal{B}_{0}}$, a restrição de $\varphi$ a $\mathcal{B}_{0}$, é um homomorfismo ou um antihomomorfismo.

Demonstração. Dados $x \in \mathcal{B}$ e $x_{0} \in \mathcal{B}_{0}$, temos

$$
\begin{aligned}
2 x_{0} \circ\left(x_{0} \circ x\right) & =x_{0} \circ\left(x_{0} x+x x_{0}\right) \\
& =x_{0} x x_{0}+\frac{1}{2}\left(x_{0}^{2} x+x x_{0}^{2}\right) \\
& =x_{0} x x_{0}+x_{0}^{2} \circ x .
\end{aligned}
$$

Assim,

$$
\begin{aligned}
\varphi\left(x_{0} x x_{0}\right)= & 2 \varphi\left(x_{0} \circ\left(x_{0} \circ x\right)\right)-\varphi\left(x_{0}^{2} \circ x\right) \\
= & 2 \varphi\left(x_{0}\right) \circ\left(\varphi\left(x_{0}\right) \circ \varphi(x)\right)-\varphi\left(x_{0}^{2}\right) \circ \varphi(x) \\
= & \frac{1}{2}\left(\varphi\left(x_{0}\right)\left(\varphi\left(x_{0}\right) \circ \varphi(x)\right)+\left(\varphi\left(x_{0}\right) \circ \varphi(x)\right) \varphi\left(x_{0}\right)\right)-\frac{1}{2}\left(\varphi\left(x_{0}^{2}\right) \varphi(x)+\varphi(x) \varphi\left(x_{0}^{2}\right)\right) \\
= & \frac{1}{2}\left(\varphi\left(x_{0}\right)^{2} \varphi(x)+\varphi\left(x_{0}\right) \varphi(x) \varphi\left(x_{0}\right)+\varphi\left(x_{0}\right) \varphi(x) \varphi\left(x_{0}\right)+\varphi(x) \varphi\left(x_{0}\right)^{2}\right. \\
& \left.-\varphi\left(x_{0}^{2}\right) \varphi(x)+\varphi(x) \varphi\left(x_{0}^{2}\right)\right) \\
= & \frac{1}{2}\left(\varphi\left(x_{0}\right)^{2} \varphi(x)+\varphi(x) \varphi\left(x_{0}\right)^{2}-\varphi\left(x_{0}^{2}\right) \varphi(x)+\varphi(x) \varphi\left(x_{0}^{2}\right)\right)+\varphi\left(x_{0}\right) \varphi(x) \varphi\left(x_{0}\right) \\
= & \varphi\left(x_{0}\right) \varphi(x) \varphi\left(x_{0}\right)
\end{aligned}
$$

pois $\varphi\left(x_{0} \circ x_{0}\right)=\varphi\left(x_{0}\right) \circ \varphi\left(x_{0}\right)$ implica $\varphi\left(x_{0}^{2}\right)=\varphi\left(x_{0}\right)^{2}$. Portanto, está provada a seguinte igualdade

$$
\varphi\left(x_{0} x x_{0}\right)=\varphi\left(x_{0}\right) \varphi(x) \varphi\left(x_{0}\right)
$$

cuja linearização em $x_{0}$ resulta em

$$
\varphi\left(x_{0} x y_{0}+y_{0} x x_{0}\right)=\varphi\left(x_{0}\right) \varphi(x) \varphi\left(y_{0}\right)+\varphi\left(y_{0}\right) \varphi(x) \varphi\left(x_{0}\right),
$$


para todos $x_{0}, y_{0} \in \mathcal{B}_{0}$ e $x \in \mathcal{B}$. Agora, considerando o elemento $W=\varphi\left(x_{0} y_{0} x y_{0} x_{0}+y_{0} x_{0} x x_{0} y_{0}\right)$, então, por (4.10), temos

$$
\begin{aligned}
W & =\varphi\left(x_{0}\left(y_{0} x y_{0}\right) x_{0}\right)+\varphi\left(y_{0}\left(x_{0} x x_{0}\right) y_{0}\right) \\
& =\varphi\left(x_{0}\right) \varphi\left(y_{0} x y_{0}\right) \varphi\left(x_{0}\right)+\varphi\left(y_{0}\right) \varphi\left(x_{0} x x_{0}\right) \varphi\left(y_{0}\right) \\
& =\varphi\left(x_{0}\right) \varphi\left(y_{0}\right) \varphi(x) \varphi\left(y_{0}\right) \varphi\left(x_{0}\right)+\varphi\left(y_{0}\right) \varphi\left(x_{0}\right) \varphi(x) \varphi\left(x_{0}\right) \varphi\left(y_{0}\right),
\end{aligned}
$$

e por outro lado, por (4.11), obtemos

$$
\begin{aligned}
W & =\varphi\left(\left(x_{0} y_{0}\right) x\left(y_{0} x_{0}\right)+\left(y_{0} x_{0}\right) x\left(x_{0} y_{0}\right)\right) \\
& =\varphi\left(x_{0} y_{0}\right) \varphi(x) \varphi\left(y_{0} x_{0}\right)+\varphi\left(y_{0} x_{0}\right) \varphi(x) \varphi\left(x_{0} y_{0}\right)
\end{aligned}
$$

Consequentemente, como $\varphi\left(x_{0} y_{0}\right)+\varphi\left(y_{0} x_{0}\right)=\varphi\left(x_{0}\right) \varphi\left(y_{0}\right)+\varphi\left(y_{0}\right) \varphi\left(x_{0}\right)$, o segundo bloco de igualdades se tornam

$$
\begin{aligned}
W= & \varphi\left(x_{0} y_{0}\right) \varphi(x) \varphi\left(x_{0}\right) \varphi\left(y_{0}\right)+\varphi\left(x_{0} y_{0}\right) \varphi(x) \varphi\left(y_{0}\right) \varphi\left(x_{0}\right)-\varphi\left(x_{0} y_{0}\right) \varphi(x) \varphi\left(x_{0} y_{0}\right) \\
& +\varphi\left(x_{0}\right) \varphi\left(y_{0}\right) \varphi(x) \varphi\left(x_{0} y_{0}\right)+\varphi\left(y_{0}\right) \varphi\left(x_{0}\right) \varphi(x) \varphi\left(x_{0} y_{0}\right)-\varphi\left(x_{0} y_{0}\right) \varphi(x) \varphi\left(x_{0} y_{0}\right),
\end{aligned}
$$

e comparando com o primeiro bloco de expressões para $W$, chegamos a

$$
\tau\left(x_{0}, y_{0}\right) \varphi(x) \omega\left(x_{0}, y_{0}\right)+\omega\left(x_{0}, y_{0}\right) \varphi(x) \tau\left(x_{0}, y_{0}\right)=0
$$

para todos $x_{0}, y_{0} \in \mathcal{B}_{0}$ e $x \in \mathcal{B}$. Nosso objetivo é mostrar que ocorre $\tau\left(\mathcal{B}_{0}, \mathcal{B}_{0}\right)=0$ ou $\omega\left(\mathcal{B}_{0}, \mathcal{B}_{0}\right)=0$.

Para isso, sejam $x_{0}, y_{0} \in \mathcal{B}_{0}$, e denotemos $\tau_{0}=\tau\left(x_{0}, y_{0}\right)$ e $\omega_{0}=\omega\left(x_{0}, y_{0}\right)$. Em vista de $\varphi$ ser sobrejetor, a igualdade (4.12) pode ser escrita como $\tau_{0} a \omega_{0}+\omega_{0} a \tau_{0}=0$, para todo $a \in \mathcal{A}$. Desse modo, obtemos

$$
\tau_{0} a\left(\omega_{0} b \tau_{0}\right)=-\tau_{0}\left(a \tau_{0} b\right) \omega_{0}=\left(\omega_{0} a \tau_{0}\right) b \tau_{0}=-\tau_{0} a \omega_{0} b \tau_{0},
$$

para quaisquer $a, b \in \mathcal{A}$, o que implica $\tau_{0} \mathcal{A} \omega_{0} \mathcal{A} \tau_{0}=0$, pois $\frac{1}{2} \in \Phi$. Agora, pela condição (ii) das hipóteses, temos $\tau_{0}, \omega_{0} \in \mathcal{A}_{0}$, e portanto pela condição (iii), concluímos $\tau_{0}=0$ ou $\omega_{0}=0$. Logo, para cada par de elementos $x_{0}, y_{0} \in \mathcal{B}_{0}$, temos $\tau\left(x_{0}, y_{0}\right)=0$ ou $\omega\left(x_{0}, y_{0}\right)=0$.

Prosseguindo, fixemos $x_{0} \in \mathcal{B}_{0}$ e definamos os conjuntos $\mathcal{B}_{0}^{1}=\left\{y_{0} \in \mathcal{B}_{0} \mid \tau\left(x_{0}, y_{0}\right)=0\right\}$ e $\mathcal{B}_{0}^{2}=\left\{y_{0} \in \mathcal{B}_{0} \mid \omega\left(x_{0}, y_{0}\right)=0\right\}$. Note que $\mathcal{B}_{0}^{1}$ e $\mathcal{B}_{0}^{2}$ são subgrupos aditivos de $\mathcal{B}_{0}$ tais que $\mathcal{B}_{0}=\mathcal{B}_{0}^{1} \cup \mathcal{B}_{0}^{2}$. Assim, como um grupo não pode ser escrito como união de dois subgrupos próprios, concluímos $\mathcal{B}_{0}=\mathcal{B}_{0}^{1}$ ou $\mathcal{B}_{0}=\mathcal{B}_{0}^{2}$, isto é, $\tau\left(x_{0}, \mathcal{B}_{0}\right)=0$ ou $\omega\left(x_{0}, \mathcal{B}_{0}\right)=0$. Analogamente, definindo os conjuntos $\mathcal{B}_{0}^{3}=\left\{x_{0} \in \mathcal{B}_{0} \mid \tau\left(x_{0}, \mathcal{B}_{0}\right)=0\right\}$ e $\mathcal{B}_{0}^{4}=\left\{x_{0} \in \mathcal{B}_{0} \mid \omega\left(x_{0}, \mathcal{B}_{0}\right)=0\right\}$, temos que $\mathcal{B}_{0}^{3}$ e $\mathcal{B}_{0}^{4}$ são subgrupos aditivos de $\mathcal{B}_{0}$ tais que $\mathcal{B}_{0}=\mathcal{B}_{0}^{3} \cup \mathcal{B}_{0}^{4}$, e novamente concluímos $\mathcal{B}_{0}=\mathcal{B}_{0}^{3}$ ou $\mathcal{B}_{0}=\mathcal{B}_{0}^{4}$, isto é, $\tau\left(\mathcal{B}_{0}, \mathcal{B}_{0}\right)=0$ ou $\omega\left(\mathcal{B}_{0}, \mathcal{B}_{0}\right)=0$.

A partir de agora, até o final dessa seção, a aplicação $\varphi$ denotará um superhomomorfismo de Jordan sobrejetor de uma superálgebra associativa $\mathcal{B}=\mathcal{B}_{0} \oplus \mathcal{B}_{1}$ em uma superálgebra associativa prima $\mathcal{A}=\mathcal{A}_{0} \oplus \mathcal{A}_{1}$, e $Z(\mathcal{A})$ e $Z\left(\mathcal{A}_{0}\right)$ serão os centros das álgebras $\mathcal{A}$ e $\mathcal{A}_{0}$, respectivamente. Além disso, assumiremos que $\mathcal{A}_{0}$ não é comutativa, ou seja, $Z\left(\mathcal{A}_{0}\right) \neq \mathcal{A}_{0}$. Desse modo, 


$$
\begin{aligned}
\tau\left(x, x_{0}\right)+\tau\left(x_{0}, x\right) & =\varphi\left(x x_{0}\right)-\varphi(x) \varphi\left(x_{0}\right)+\varphi\left(x_{0} x\right)-\varphi\left(x_{0}\right) \varphi(x) \\
& =2\left(\varphi\left(x \circ_{s} x_{0}\right)-\varphi(x) \circ_{s} \varphi\left(x_{0}\right)\right)=0,
\end{aligned}
$$

para todos $x \in \mathcal{B}, x_{0} \in \mathcal{B}_{0}$, isto é, $\tau\left(x, x_{0}\right)=-\tau\left(x_{0}, x\right)$. Analogamente, $\omega\left(x, x_{0}\right)=-\omega\left(x_{0}, x\right)$.

Além disso,

$$
\begin{aligned}
\tau\left(x_{1}, y_{1}\right)-\tau\left(y_{1}, x_{1}\right) & =\varphi\left(x_{1} y_{1}\right)-\varphi\left(x_{1}\right) \varphi\left(y_{1}\right)-\varphi\left(y_{1} x_{1}\right)+\varphi\left(y_{1}\right) \varphi\left(x_{1}\right) \\
& =2\left(\varphi\left(x_{1} \circ_{s} y_{1}\right)-\varphi\left(x_{1}\right) \circ_{s} \varphi\left(y_{1}\right)\right)=0,
\end{aligned}
$$

para quaisquer $x_{1}, y_{1} \in \mathcal{B}_{1}$, ou seja, $\tau\left(x_{1}, y_{1}\right)=\tau\left(y_{1}, x_{1}\right)$. Analogamente, $\rho\left(x_{1}, y_{1}\right)=\rho\left(y_{1}, x_{1}\right)$.

Prosseguindo, dados $y=y_{0}+y_{1} \in \mathcal{B}$ e $x_{1} \in \mathcal{B}_{1}$, temos

$$
\begin{aligned}
2\left[x_{1}, x_{1} \circ y_{0}\right]+2 x_{1} \circ\left[x_{1}, y_{1}\right]= & x_{1}\left(x_{1} y_{0}+y_{0} x_{1}\right)-\left(x_{1} y_{0}+y_{0} x_{1}\right) x_{1}+x_{1}\left(x_{1} y_{1}-y_{1} x_{1}\right) \\
& +\left(x_{1} y_{1}-y_{1} x_{1}\right) x_{1} \\
= & x_{1}^{2} y_{0}-y_{0} x_{1}^{2}+x_{1}^{2} y_{1}-y_{1} x_{1}^{2} \\
= & {\left[x_{1}^{2}, y\right] . }
\end{aligned}
$$

Portanto, pela definição de superhomomorfismo de Jordan, segue

$$
\begin{aligned}
\varphi\left(\left[x_{1}^{2}, y\right]\right)= & 2 \varphi\left(\left[x_{1}, x_{1} \circ y_{0}\right]\right)+2 \varphi\left(x_{1} \circ\left[x_{1}, y_{1}\right]\right) \\
= & \varphi\left(x_{1} \circ_{s}\left(x_{1} \circ_{s} y_{0}\right)\right)+\varphi\left(x_{1} \circ_{s}\left(x_{1} \circ_{s} y_{1}\right)\right) \\
= & \varphi\left(x_{1}\right) \circ_{s}\left(\varphi\left(x_{1}\right) \circ_{s} \varphi\left(y_{0}\right)\right)+\varphi\left(x_{1}\right) \circ_{s}\left(\varphi\left(x_{1}\right) \circ_{s} \varphi\left(y_{1}\right)\right) \\
= & \varphi\left(x_{1}\right)\left(\varphi\left(x_{1}\right) \varphi\left(y_{0}\right)+\varphi\left(y_{0}\right) \varphi\left(x_{1}\right)\right)-\left(\varphi\left(x_{1}\right) \varphi\left(y_{0}\right)+\varphi\left(y_{0}\right) \varphi\left(x_{1}\right)\right) \varphi\left(x_{1}\right) \\
& +\varphi\left(x_{1}\right)\left(\varphi\left(x_{1}\right) \varphi\left(y_{1}\right)-\varphi\left(y_{1}\right) \varphi\left(x_{1}\right)\right)+\left(\varphi\left(x_{1}\right) \varphi\left(y_{1}\right)-\varphi\left(y_{1}\right) \varphi\left(x_{1}\right)\right) \varphi\left(x_{1}\right) \\
= & \varphi\left(x_{1}\right)^{2} \varphi\left(y_{0}\right)-\varphi\left(y_{0}\right) \varphi\left(x_{1}\right)^{2}+\varphi\left(x_{1}\right)^{2} \varphi\left(y_{1}\right)-\varphi\left(y_{1}\right) \varphi\left(x_{1}\right)^{2} \\
= & {\left[\varphi\left(x_{1}\right)^{2}, \varphi(y)\right], }
\end{aligned}
$$

Logo, podemos concluir

$$
\varphi\left(\left[x_{1}^{2}, y\right]\right)=\left[\varphi\left(x_{1}\right)^{2}, \varphi(y)\right]
$$

para todos $x_{1} \in \mathcal{B}_{1}, y \in \mathcal{B}$.

Lema 4.2.1. A aplicaç ão $\left.\varphi\right|_{\mathcal{B}_{0}}$ é um homomorfismo sobrejetor ou um antihomomorfismo sobrejetor de $\mathcal{B}_{0}$ em $\mathcal{A}_{0}$.

Demonstração. Sabemos que $\varphi$ é um homomorfismo de $\Phi$-módulos que preserva a $\mathbb{Z}_{2}$-gaduação, pois $\varphi$ é superhomomorfismo de Jordan; e $\varphi$ é sobrejetor. Além disso, pela definição de $\mathbb{Z}_{2}$-graduação, as partes pares $\mathcal{B}_{0}$ de $\mathcal{B}$ e $\mathcal{A}_{0}$ de $\mathcal{A}$ são subálgebras de $\mathcal{B}$ e $\mathcal{A}$, respectivamente, tais que $\varphi\left(x \circ x_{0}\right)=$ $\varphi\left(x \circ_{s} x_{0}\right)=\varphi(x) \circ_{s} \varphi\left(x_{0}\right)=\varphi(x) \circ \varphi\left(x_{0}\right) ;$ e se $a_{0}, b_{0} \in \mathcal{A}_{0}$ são tais que $a_{0} \mathcal{A} b_{0} \mathcal{A} a_{0}=0$, então, $a_{0}=0$ ou $b_{0}=0$, visto que $\mathcal{A}$ é uma superálgebra associativa prima.

Portanto, podemos aplicar a Proposição 4.2.1, o que implica que $\left.\varphi\right|_{\mathcal{B}_{0}}$ é um homomorfismo ou um antihomomorfismo. Claramente, se $a_{0} \in \mathcal{A}_{0}$, então, como $\varphi$ é sobrejetor, existe $x=x_{0}+x_{1} \in \mathcal{B}$, onde $x_{i} \in \mathcal{B}_{i}$ com $i=0,1$, tal que $\varphi(x)=a_{0}$. Portanto, $a_{0}-\varphi\left(x_{0}\right)=\varphi\left(x_{1}\right) \in \mathcal{A}_{0} \cap \mathcal{A}_{1}=0$, de onde segue $a_{0}=\varphi\left(x_{0}\right) \in \varphi\left(\mathcal{B}_{0}\right)$.

Lema 4.2.2. Se $\left.\varphi\right|_{\mathcal{B}_{0}}$ é um homomorfismo, então, $\varphi$ é um superhomomorfismo. 
Demonstração. Primeiramente, note que por hipótese seguem

$$
\begin{aligned}
\varphi\left(\left[x_{1}^{2}, x_{0}\right]\right) & =\varphi\left(x_{1}^{2}\right) \varphi\left(x_{0}\right)-\varphi\left(x_{0}\right) \varphi\left(x_{1}^{2}\right) \\
& =\left[\varphi\left(x_{1}^{2}\right), \varphi\left(x_{0}\right)\right],
\end{aligned}
$$

para todos $x_{0} \in \mathcal{B}_{0}, x_{1} \in \mathcal{B}_{1}$ que comparando com (4.13) implica $\left[\tau\left(x_{1}, x_{1}\right), \varphi\left(x_{0}\right)\right]=0$. Assim, como $\varphi\left(\mathcal{B}_{0}\right)=\mathcal{A}_{0}$, temos $\tau\left(x_{1}, x_{1}\right) \in Z\left(\mathcal{A}_{0}\right)$, para todo $x_{1} \in \mathcal{B}_{1}$. Desse modo, linearizando a última igualdade, obtemos

$$
\left[\tau\left(x_{1}, y_{1}\right)+\tau\left(y_{1}, x_{1}\right), \varphi\left(x_{0}\right)\right]=0,
$$

o que implica

$$
\tau\left(x_{1}, y_{1}\right) \in Z\left(\mathcal{A}_{0}\right),
$$

para todos $x_{1}, y_{1} \in \mathcal{B}_{1}$, pois $\tau\left(x_{1}, y_{1}\right)=\tau\left(y_{1}, x_{1}\right)$.

Agora, consideremos o elemento $\varphi\left(x_{0} x_{1} y_{1}\right)$, onde $x_{0} \in \mathcal{B}_{0}$ e $x_{1}, y_{1} \in \mathcal{B}_{1}$. Por um lado, temos

$$
\begin{aligned}
\varphi\left(x_{0}\left(x_{1} y_{1}\right)\right) & =\varphi\left(x_{0}\right) \varphi\left(x_{1} y_{1}\right) \\
& =\varphi\left(x_{0}\right) \varphi\left(x_{1}\right) \varphi\left(y_{1}\right)+\varphi\left(x_{0}\right) \tau\left(x_{1}, y_{1}\right)
\end{aligned}
$$

e por outro lado, obtemos

$$
\begin{aligned}
\varphi\left(\left(x_{0} x_{1}\right) y_{1}\right) & =\varphi\left(x_{0} x_{1}\right) \varphi\left(y_{1}\right)+\tau\left(x_{0} x_{1}, y_{1}\right) \\
& =\varphi\left(x_{0}\right) \varphi\left(x_{1}\right) \varphi\left(y_{1}\right)+\tau\left(x_{0}, x_{1}\right) \varphi\left(y_{1}\right)+\tau\left(x_{0} x_{1}, y_{1}\right) .
\end{aligned}
$$

A comparação das igualdades encontradas, resulta em

$$
\tau\left(x_{0}, x_{1}\right) \varphi\left(y_{1}\right)=\varphi\left(x_{0}\right) \tau\left(x_{1}, y_{1}\right)-\tau\left(x_{0} x_{1}, y_{1}\right),
$$

para todos $x_{0} \in \mathcal{B}_{0}, x_{1}, y_{1} \in \mathcal{B}_{1}$.

Analogamente, considerando o elemento $\varphi\left(y_{1} x_{1} x_{0}\right)$, as seguintes igualdades são válidas

$$
\begin{gathered}
\varphi\left(\left(y_{1} x_{1}\right) x_{0}\right)=\varphi\left(y_{1} x_{1}\right) \varphi\left(x_{0}\right) \\
=\varphi\left(y_{1}\right) \varphi\left(x_{1}\right) \varphi\left(x_{0}\right)+\tau\left(y_{1}, x_{1}\right) \varphi\left(x_{0}\right) \mathrm{e} \\
\varphi\left(y_{1}\left(x_{1} x_{0}\right)\right)=\varphi\left(y_{1}\right) \varphi\left(x_{1} x_{0}\right)+\tau\left(y_{1}, x_{1} x_{0}\right) \\
=\varphi\left(y_{1}\right) \varphi\left(x_{1}\right) \varphi\left(x_{0}\right)+\varphi\left(y_{1}\right) \tau\left(x_{1}, x_{0}\right)+\tau\left(y_{1}, x_{1} x_{0}\right) .
\end{gathered}
$$

Novamente, comparando os blocos de igualdades, segue

$$
\varphi\left(y_{1}\right) \tau\left(x_{0}, x_{1}\right)=-\tau\left(x_{1}, y_{1}\right) \varphi\left(x_{0}\right)+\tau\left(x_{1} x_{0}, y_{1}\right),
$$

para todos $x_{0} \in \mathcal{B}_{0}, x_{1}, y_{1} \in \mathcal{B}_{1}$, pois $\tau\left(x_{1}, x_{0}\right)=-\tau\left(x_{0}, x_{1}\right), \tau\left(y_{1}, x_{1}\right)=\tau\left(x_{1}, y_{1}\right)$ e $\tau\left(y_{1}, x_{1} x_{0}\right)=$ $\tau\left(x_{1} x_{0}, y_{1}\right)$.

Além disso, por (4.14) e (4.15), temos

$$
\begin{aligned}
{\left[\tau\left(x_{0}, x_{1}\right) \varphi\left(y_{1}\right), \varphi\left(x_{0}\right)\right] } & =\left[\varphi\left(x_{0}\right) \tau\left(x_{1}, y_{1}\right), \varphi\left(x_{0}\right)\right]-\left[\tau\left(x_{0} x_{1}, y_{1}\right), \varphi\left(x_{0}\right)\right] \\
& =\varphi\left(x_{0}\right)\left[\tau\left(x_{1}, y_{1}\right), \varphi\left(x_{0}\right)\right]-\left[\tau\left(x_{0} x_{1}, y_{1}\right), \varphi\left(x_{0}\right)\right]=0 .
\end{aligned}
$$


Portanto, $\left[\tau\left(x_{0}, \mathcal{B}_{1}\right) \mathcal{A}_{1}, \varphi\left(x_{0}\right)\right]=0$, para todo $x_{0} \in \mathcal{B}_{0}$, pois $\varphi\left(\mathcal{B}_{1}\right)=\mathcal{A}_{1}$. Consequentemente, para quaisquer $a_{0} \in \mathcal{A}_{0}$ e $a_{1} \in \mathcal{A}_{1}$, como sabemos que vale $a_{1} a_{0} \in \mathcal{A}_{1}$, as igualdades abaixo são válidas:

$$
\begin{aligned}
\tau\left(x_{0}, x_{1}\right) a_{1}\left[a_{0}, \varphi\left(x_{0}\right)\right]= & \tau\left(x_{0}, x_{1}\right) a_{1} a_{0} \varphi\left(x_{0}\right)-\tau\left(x_{0}, x_{1}\right) a_{1} \varphi\left(x_{0}\right) a_{0} \\
= & \tau\left(x_{0}, x_{1}\right) a_{1} a_{0} \varphi\left(x_{0}\right)-\varphi\left(x_{0}\right) \tau\left(x_{0}, x_{1}\right) a_{1} a_{0}+\varphi\left(x_{0}\right) \tau\left(x_{0}, x_{1}\right) a_{1} a_{0} \\
& -\tau\left(x_{0}, x_{1}\right) a_{1} \varphi\left(x_{0}\right) a_{0} \\
= & {\left[\tau\left(x_{0}, x_{1}\right)\left(a_{1} a_{0}\right), \varphi\left(x_{0}\right)\right]-\left[\tau\left(x_{0}, x_{1}\right) a_{1}, \varphi\left(x_{0}\right)\right] a_{0}=0, }
\end{aligned}
$$

isto é,

$$
\tau\left(x_{0}, \mathcal{B}_{1}\right) \mathcal{A}_{1}\left[\mathcal{A}_{0}, \varphi\left(x_{0}\right)\right]=0
$$

para todo $x_{0} \in \mathcal{B}_{0}$.

Do mesmo modo, por (4.14) e (4.16), valem

$$
\begin{aligned}
{\left[\varphi\left(y_{1}\right) \tau\left(x_{0}, x_{1}\right), \varphi\left(x_{0}\right)\right] } & =-\left[\tau\left(x_{1}, y_{1}\right) \varphi\left(x_{0}\right), \varphi\left(x_{0}\right)\right]+\left[\tau\left(x_{1} x_{0}, y_{1}\right), \varphi\left(x_{0}\right)\right] \\
& =-\left[\tau\left(x_{1}, y_{1}\right), \varphi\left(x_{0}\right)\right] \varphi\left(x_{0}\right)+\left[\tau\left(x_{1} x_{0}, y_{1}\right), \varphi\left(x_{0}\right)\right]=0 .
\end{aligned}
$$

Logo, $\left[\mathcal{A}_{1} \tau\left(x_{0}, \mathcal{B}_{1}\right), \varphi\left(x_{0}\right)\right]=0$, para qualquer $x_{0} \in \mathcal{B}_{0}$, pois $\varphi\left(\mathcal{B}_{1}\right)=\mathcal{A}_{1}$.

Agora, dados $a_{0} \in \mathcal{A}_{0}$ e $a_{1} \in \mathcal{A}_{1}$, também temos

$$
\begin{aligned}
{\left[a_{0}, \varphi\left(x_{0}\right)\right] a_{1} \tau\left(x_{0}, x_{1}\right)=} & a_{0} \varphi\left(x_{0}\right) a_{1} \tau\left(x_{0}, x_{1}\right)-\varphi\left(x_{0}\right) a_{0} a_{1} \tau\left(x_{0}, x_{1}\right) \\
= & a_{0} \varphi\left(x_{0}\right) a_{1} \tau\left(x_{0}, x_{1}\right)-a_{0} a_{1} \tau\left(x_{0}, x_{1}\right) \varphi\left(x_{0}\right)+a_{0} a_{1} \tau\left(x_{0}, x_{1}\right) \varphi\left(x_{0}\right) \\
& -\varphi\left(x_{0}\right) a_{0} a_{1} \tau\left(x_{0}, x_{1}\right) \\
= & -a_{0}\left[a_{1} \tau\left(x_{0}, x_{1}\right), \varphi\left(x_{0}\right)\right]+\left[\left(a_{0} a_{1}\right) \tau\left(x_{0}, x_{1}\right), \varphi\left(x_{0}\right)\right]=0,
\end{aligned}
$$

ou seja,

$$
\left[\mathcal{A}_{0}, \varphi\left(x_{0}\right)\right] \mathcal{A}_{1} \tau\left(x_{0}, \mathcal{B}_{1}\right)=0 .
$$

para todo $x_{0} \in \mathcal{B}_{0}$. Assim, como $\tau\left(x_{0}, \mathcal{B}_{1}\right) \subseteq \mathcal{A}_{1}$ e $\left[\mathcal{A}_{0}, \varphi\left(x_{0}\right)\right] \subseteq \mathcal{A}_{0}$, por (2) do Lema 1.2.5, (4.17) e (4.18) concluímos $\tau\left(x_{0}, \mathcal{B}_{1}\right)=0$ ou $\varphi\left(x_{0}\right) \in Z\left(\mathcal{A}_{0}\right)$, para cada $x_{0} \in \mathcal{B}_{0}$.

Pela mesma técnica usada na demonstração da Proposição 4.2.1, definimos os conjuntos $\mathcal{B}_{0}^{\prime}=$ $\left\{x_{0} \in \mathcal{B}_{0} \mid \tau\left(x_{0}, \mathcal{B}_{1}\right)=0\right\}$ e $\mathcal{B}_{0}^{\prime \prime}=\left\{x_{0} \in \mathcal{B}_{0} \mid \varphi\left(x_{0}\right) \in Z\left(\mathcal{A}_{0}\right)\right\}$, os quais são subgrupos do grupo aditivo $\mathcal{B}_{0}$ tais que $\mathcal{B}_{0}=\mathcal{B}_{0}^{\prime} \cup \mathcal{B}_{0}^{\prime \prime}$, o que implica $\mathcal{B}_{0}=\mathcal{B}_{0}^{\prime}$ ou $\mathcal{B}_{0}=\mathcal{B}_{0}^{\prime \prime}$, isto é, $\tau\left(\mathcal{B}_{0}, \mathcal{B}_{1}\right)=0$ ou $\varphi\left(\mathcal{B}_{0}\right) \subseteq Z\left(\mathcal{A}_{0}\right)$. Porém, o segundo caso não ocorre, pois $\varphi\left(\mathcal{B}_{0}\right)=\mathcal{A}_{0}$ e estamos assumindo que $\mathcal{A}_{0}$ não é comutativa. Portanto, $\tau\left(\mathcal{B}_{0}, \mathcal{B}_{1}\right)=0$; e consequentemente, $\tau\left(\mathcal{B}_{1}, \mathcal{B}_{0}\right)=0$.

Dados $x_{1}, y_{1} \in \mathcal{B}_{1}$, temos $x_{1}^{2} \in \mathcal{B}_{0}$,

$$
\begin{aligned}
\varphi\left(\left[x_{1}^{2}, y_{1}\right]\right) & =\varphi\left(x_{1}^{2} y_{1}\right)-\varphi\left(y_{1} x_{1}^{2}\right) \\
& =\varphi\left(x_{1}^{2}\right) \varphi\left(y_{1}\right)-\varphi\left(y_{1}\right) \varphi\left(x_{1}^{2}\right) \\
& =\left[\varphi\left(x_{1}^{2}\right), \varphi\left(y_{1}\right)\right]
\end{aligned}
$$

e $\varphi\left(\left[x_{1}^{2}, y_{1}\right]\right)=\left[\varphi\left(x_{1}\right)^{2}, \varphi\left(y_{1}\right)\right]$, por (4.13). Desse modo, vale $\left[\tau\left(x_{1}, x_{1}\right), \varphi\left(y_{1}\right)\right]=0$, cuja linearização resulta em

$$
\left[\tau\left(x_{1}, z_{1}\right), \varphi\left(y_{1}\right)\right]+\left[\tau\left(z_{1}, x_{1}\right), \varphi\left(y_{1}\right)\right]=0
$$


de onde concluímos $\left[\tau\left(x_{1}, z_{1}\right), \varphi\left(y_{1}\right)\right]=0$, pois $\tau\left(x_{1}, z_{1}\right)=\tau\left(z_{1}, x_{1}\right)$. Portanto, como sabemos que ocorre $\varphi\left(\mathcal{B}_{1}\right)=\mathcal{A}_{1}$, temos $\left[\tau\left(x_{1}, z_{1}\right), \mathcal{A}_{1}\right]=0$, o que implica $\tau\left(x_{1}, z_{1}\right) \in Z(\mathcal{A})$, para todos $x_{1}, z_{1} \in \mathcal{B}_{1}$, por (4.14). Assim, a igualdade de (4.16) nos mostra $\tau\left(x_{1}, y_{1}\right) \varphi\left(x_{0}\right)=\tau\left(x_{1} x_{0}, y_{1}\right) \in Z(\mathcal{A})$, para todos $x_{1}, y_{1} \in \mathcal{B}_{1}, x_{0} \in \mathcal{B}_{0}$. Logo, $\tau\left(\mathcal{B}_{1}, \mathcal{B}_{1}\right) \mathcal{A}_{0} \subseteq Z(\mathcal{A})$, pois $\varphi\left(\mathcal{B}_{0}\right)=\mathcal{A}_{0}$. Consequentemente, valem as seguintes igualdades

$$
\begin{aligned}
\tau\left(x_{1}, y_{1}\right)\left[a_{0}, b_{0}\right] & =\left(\tau\left(x_{1}, y_{1}\right)\right) a_{0} b_{0}-\left(\tau\left(x_{1}, y_{1}\right) b_{0}\right) a_{0} \\
& =a_{0} \tau\left(x_{1}, y_{1}\right) b_{0}-a_{0} \tau\left(x_{1}, y_{1}\right) b_{0}=0
\end{aligned}
$$

para todos $x_{1}, y_{1} \in \mathcal{B}_{1}$ e $a_{0}, b_{0} \in \mathcal{A}_{0}$.

Finalmente, como $\left[\mathcal{A}_{0}, \mathcal{A}_{0}\right] \neq 0$, existem $a_{0}, b_{0} \in \mathcal{A}_{0}$ tais que $\left[a_{0}, b_{0}\right] \neq 0$. Assim, se $a \in \mathcal{A}$ e $x_{1}, y_{1} \in \mathcal{B}_{1}$, então,

$$
\tau\left(x_{1}, y_{1}\right) a\left[a_{0}, b_{0}\right]=a \tau\left(x_{1}, y_{1}\right)\left[a_{0}, b_{0}\right]=0,
$$

isto é, $\tau\left(x_{1}, y_{1}\right) \mathcal{A}\left[a_{0}, b_{0}\right]=0$, o que implica $\tau\left(x_{1}, y_{1}\right)=0$, pois $\mathcal{A}$ é uma superálgebra associativa prima. Desse modo, $\tau\left(\mathcal{B}_{1}, \mathcal{B}_{1}\right)=0$, e por hipótese, como $\left.\varphi\right|_{\mathcal{B}_{0}}$ é homomorfismo, também temos $\tau\left(\mathcal{B}_{0}, \mathcal{B}_{0}\right)=0$. Logo, $\tau(\mathcal{B}, \mathcal{B})=0$, ou seja, $\varphi$ é um superhomomorfismo.

Lema 4.2.3. Se $\left.\varphi\right|_{\mathcal{B}_{0}}$ é um antihomomorfismo, então, $\varphi$ é um superantihomomorfismo.

Demonstração. Inicialmente, por hipótese, note

$$
\begin{aligned}
\varphi\left(\left[x_{1}^{2}, x_{0}\right]\right) & =\varphi\left(x_{0}\right) \varphi\left(x_{1}^{2}\right)-\varphi\left(x_{1}^{2}\right) \varphi\left(x_{0}\right) \\
& =\left[-\varphi\left(x_{1}^{2}\right), \varphi\left(x_{0}\right)\right],
\end{aligned}
$$

para todos $x_{0} \in \mathcal{B}_{0}, x_{1} \in \mathcal{B}_{1}$, que comparando com (4.13) implica $\left[\rho\left(x_{1}, x_{1}\right), \varphi\left(x_{0}\right)\right]=0$. Assim, como $\varphi\left(\mathcal{B}_{0}\right)=\mathcal{A}_{0}$, temos $\rho\left(x_{1}, x_{1}\right) \in Z\left(\mathcal{A}_{0}\right)$, para todo $x_{1} \in \mathcal{B}_{1}$. Desse modo, linearizando a última igualdade, obtemos

$$
\left[\rho\left(x_{1}, y_{1}\right)+\rho\left(y_{1}, x_{1}\right), \varphi\left(x_{0}\right)\right]=0,
$$

o que implica

$$
\rho\left(x_{1}, y_{1}\right) \in Z\left(\mathcal{A}_{0}\right),
$$

para todos $x_{1}, y_{1} \in \mathcal{B}_{1}$, pois $\rho\left(x_{1}, y_{1}\right)=\rho\left(y_{1}, x_{1}\right)$.

Agora, consideremos o elemento $\varphi\left(x_{0} x_{1} y_{1}\right)$, onde $x_{0} \in \mathcal{B}_{0}$ e $x_{1}, y_{1} \in \mathcal{B}_{1}$. Por um lado, temos

$$
\begin{aligned}
\varphi\left(x_{0}\left(x_{1} y_{1}\right)\right) & =\varphi\left(x_{1} y_{1}\right) \varphi\left(x_{0}\right) \\
& =-\varphi\left(y_{1}\right) \varphi\left(x_{1}\right) \varphi\left(x_{0}\right)+\rho\left(x_{1}, y_{1}\right) \varphi\left(x_{0}\right),
\end{aligned}
$$

e por outro lado, obtemos

$$
\begin{aligned}
\varphi\left(\left(x_{0} x_{1}\right) y_{1}\right) & =-\varphi\left(y_{1}\right) \varphi\left(x_{0} x_{1}\right)+\rho\left(x_{0} x_{1}, y_{1}\right) \\
& =-\varphi\left(y_{1}\right) \varphi\left(x_{1}\right) \varphi\left(x_{0}\right)-\varphi\left(y_{1}\right) \omega\left(x_{0}, x_{1}\right)+\rho\left(x_{0} x_{1}, y_{1}\right) .
\end{aligned}
$$

A comparação das igualdades encontradas, resulta em

$$
\varphi\left(y_{1}\right) \omega\left(x_{0}, x_{1}\right)=-\rho\left(x_{1}, y_{1}\right) \varphi\left(x_{0}\right)+\rho\left(x_{0} x_{1}, y_{1}\right)
$$

para todos $x_{0} \in \mathcal{B}_{0}, x_{1}, y_{1} \in \mathcal{B}_{1}$. 
Analogamente, considerando o elemento $\varphi\left(y_{1} x_{1} x_{0}\right)$, as seguintes igualdades são válidas:

$$
\begin{gathered}
\varphi\left(\left(y_{1} x_{1}\right) x_{0}\right)=\varphi\left(x_{0}\right) \varphi\left(y_{1} x_{1}\right) \\
=-\varphi\left(x_{0}\right) \varphi\left(x_{1}\right) \varphi\left(y_{1}\right)+\varphi\left(x_{0}\right) \rho\left(y_{1}, x_{1}\right) \mathrm{e} \\
\varphi\left(y_{1}\left(x_{1} x_{0}\right)\right)=-\varphi\left(x_{1} x_{0}\right) \varphi\left(y_{1}\right)+\rho\left(y_{1}, x_{1} x_{0}\right) \\
=-\varphi\left(x_{0}\right) \varphi\left(x_{1}\right) \varphi\left(y_{1}\right)-\omega\left(x_{1}, x_{0}\right) \varphi\left(y_{1}\right)+\rho\left(y_{1}, x_{1} x_{0}\right) .
\end{gathered}
$$

Novamente, comparando os blocos de igualdades, segue

$$
\omega\left(x_{0}, x_{1}\right) \varphi\left(y_{1}\right)=\varphi\left(x_{0}\right) \rho\left(x_{1}, y_{1}\right)-\rho\left(x_{1} x_{0}, y_{1}\right)
$$

para todos $x_{0} \in \mathcal{B}_{0}, x_{1}, y_{1} \in \mathcal{B}_{1}$, pois $\omega\left(x_{1}, x_{0}\right)=-\omega\left(x_{0}, x_{1}\right), \rho\left(y_{1}, x_{1}\right)=\rho\left(x_{1}, y_{1}\right)$ e $\rho\left(y_{1}, x_{1} x_{0}\right)=$ $\rho\left(x_{1} x_{0}, y_{1}\right)$.

Além disso, por (4.19) e (4.20), temos

$$
\begin{aligned}
{\left[\varphi\left(y_{1}\right) \omega\left(x_{0}, x_{1}\right), \varphi\left(x_{0}\right)\right] } & =-\left[\rho\left(x_{1}, y_{1}\right) \varphi\left(x_{0}\right), \varphi\left(x_{0}\right)\right]+\left[\rho\left(x_{0} x_{1}, y_{1}\right), \varphi\left(x_{0}\right)\right] \\
& =-\left[\rho\left(x_{1}, y_{1}\right), \varphi\left(x_{0}\right)\right] \varphi\left(x_{0}\right)-\left[\rho\left(x_{0} x_{1}, y_{1}\right), \varphi\left(x_{0}\right)\right]=0 .
\end{aligned}
$$

Portanto, $\left[\mathcal{A}_{1} \omega\left(x_{0}, \mathcal{B}_{1}\right), \varphi\left(x_{0}\right)\right]=0$, para todo $x_{0} \in \mathcal{B}_{0}$. Consequentemente, para quaisquer $a_{0} \in \mathcal{A}_{0}$ e $a_{1} \in \mathcal{A}_{1}$, como sabemos que vale $a_{1} a_{0} \in \mathcal{A}_{1}$, as próximas igualdades são válidas:

$$
\begin{aligned}
{\left[a_{0}, \varphi\left(x_{0}\right)\right] a_{1} \omega\left(x_{0}, x_{1}\right)=} & a_{0} \varphi\left(x_{0}\right) a_{1} \omega\left(x_{0}, x_{1}\right)-\varphi\left(x_{0}\right) a_{0} a_{1} \omega\left(x_{0}, x_{1}\right) \\
= & a_{0} \varphi\left(x_{0}\right) a_{1} \omega\left(x_{0}, x_{1}\right)-a_{0} a_{1} \omega\left(x_{0}, x_{1}\right) \varphi\left(x_{0}\right)+a_{0} a_{1} \omega\left(x_{0}, x_{1}\right) \varphi\left(x_{0}\right) \\
& -\varphi\left(x_{0}\right) a_{0} a_{1} \omega\left(x_{0}, x_{1}\right) \\
= & -a_{0}\left[a_{1} \omega\left(x_{0}, x_{1}\right), \varphi\left(x_{0}\right)\right]-\left[\left(a_{0} a_{1}\right) \omega\left(x_{0}, x_{1}\right), \varphi\left(x_{0}\right)\right]=0,
\end{aligned}
$$

isto é,

$$
\left[\mathcal{A}_{0}, \varphi\left(x_{0}\right)\right] \mathcal{A}_{1} \omega\left(x_{0}, \mathcal{B}_{1}\right)=0,
$$

para todo $x_{0} \in \mathcal{B}_{0}$. Do mesmo modo, por (4.19) e (4.21), seguem

$$
\begin{aligned}
{\left[\omega\left(x_{0}, x_{1}\right) \varphi\left(y_{1}\right), \varphi\left(x_{0}\right)\right] } & =\left[\varphi\left(x_{0}\right) \rho\left(x_{1}, y_{1}\right), \varphi\left(x_{0}\right)\right]-\left[\rho\left(x_{1} x_{0}, y_{1}\right), \varphi\left(x_{0}\right)\right] \\
& =\varphi\left(x_{0}\right)\left[\rho\left(x_{1}, y_{1}\right), \varphi\left(x_{0}\right)\right]-\left[\rho\left(x_{1} x_{0}, y_{1}\right), \varphi\left(x_{0}\right)\right]=0 .
\end{aligned}
$$

Logo, $\left[\omega\left(x_{0}, \mathcal{B}_{1}\right) \mathcal{A}_{1}, \varphi\left(x_{0}\right)\right]=0$, para qualquer $x_{0} \in \mathcal{B}_{0}$. Agora, dados $a_{0} \in \mathcal{A}_{0}$ e $a_{1} \in \mathcal{A}_{1}$, também temos

$$
\begin{aligned}
\omega\left(x_{0}, x_{1}\right) a_{1}\left[a_{0}, \varphi\left(x_{0}\right)\right]= & \omega\left(x_{0}, x_{1}\right) a_{1} a_{0} \varphi\left(x_{0}\right)-\omega\left(x_{0}, x_{1}\right) a_{1} \varphi\left(x_{0}\right) a_{0} \\
= & \omega\left(x_{0}, x_{1}\right) a_{1} a_{0} \varphi\left(x_{0}\right)-\varphi\left(x_{0}\right) \omega\left(x_{0}, x_{1}\right) a_{1} a_{0}+\varphi\left(x_{0}\right) \omega\left(x_{0}, x_{1}\right) a_{1} a_{0} \\
& -\omega\left(x_{0}, x_{1}\right) a_{1} \varphi\left(x_{0}\right) a_{0} \\
= & {\left[\omega\left(x_{0}, x_{1}\right)\left(a_{1} a_{0}\right), \varphi\left(x_{0}\right)\right]-\left[\omega\left(x_{0}, x_{1}\right) a_{1}, \varphi\left(x_{0}\right)\right] a_{0}=0, }
\end{aligned}
$$

ou seja,

$$
\omega\left(x_{0}, \mathcal{B}_{1}\right) \mathcal{A}_{1}\left[\mathcal{A}_{0}, \varphi\left(x_{0}\right)\right]=0,
$$

para todo $x_{0} \in \mathcal{B}_{0}$. Assim, como $\omega\left(x_{0}, \mathcal{B}_{1}\right) \subseteq \mathcal{A}_{1}$ e $\left[\mathcal{A}_{0}, \varphi\left(x_{0}\right)\right] \subseteq \mathcal{A}_{0}$, por (2) do Lema 1.2.5, (4.22) 
e (4.23) concluímos $\omega\left(x_{0}, \mathcal{B}_{1}\right)=0$ ou $\varphi\left(x_{0}\right) \in Z\left(\mathcal{A}_{0}\right)$, para cada $x_{0} \in \mathcal{B}_{0}$. Desse modo, definindo os conjuntos $\mathcal{B}_{0}^{*}=\left\{x_{0} \in \mathcal{B}_{0} \mid \omega\left(x_{0}, \mathcal{B}_{1}\right)=0\right\}$ e $\mathcal{B}_{0}^{* *}=\left\{x_{0} \in \mathcal{B}_{0} \mid \varphi\left(x_{0}\right) \in Z\left(\mathcal{A}_{0}\right)\right\}$, os quais são subgrupos do grupo aditivo $\mathcal{B}_{0}$ tais que $\mathcal{B}_{0}=\mathcal{B}_{0}^{*} \cup \mathcal{B}_{0}^{* *}$, temos $\omega\left(\mathcal{B}_{0}, \mathcal{B}_{1}\right)=0$ ou $\varphi\left(\mathcal{B}_{0}\right) \subseteq Z\left(\mathcal{A}_{0}\right)$. Entretanto, o segundo caso não acontece, pois $\varphi\left(\mathcal{B}_{0}\right)=\mathcal{A}_{0}$ e estamos assumindo que $\mathcal{A}_{0}$ não é comutativa. Portanto, $\omega\left(\mathcal{B}_{0}, \mathcal{B}_{1}\right)=0$, e consequentemente, também temos $\omega\left(\mathcal{B}_{1}, \mathcal{B}_{0}\right)=0$.

Dados $x_{1}, y_{1} \in \mathcal{B}_{1}$, temos $x_{1}^{2} \in \mathcal{B}_{0}$,

$$
\begin{aligned}
\varphi\left(\left[x_{1}^{2}, y_{1}\right]\right) & =\varphi\left(x_{1}^{2} y_{1}\right)-\varphi\left(y_{1} x_{1}^{2}\right) \\
& =\varphi\left(y_{1}\right) \varphi\left(x_{1}^{2}\right)-\varphi\left(x_{1}^{2}\right) \varphi\left(y_{1}\right) \\
& =\left[-\varphi\left(x_{1}^{2}\right), \varphi\left(y_{1}\right)\right],
\end{aligned}
$$

e $\varphi\left(\left[x_{1}^{2}, y_{1}\right]\right)=\left[\varphi\left(x_{1}\right)^{2}, \varphi\left(y_{1}\right)\right]$, logo $\left[\rho\left(x_{1}, x_{1}\right), \varphi\left(y_{1}\right)\right]=0$, por (4.13), cuja linearização resulta em

$$
\left[\rho\left(x_{1}, z_{1}\right), \varphi\left(y_{1}\right)\right]+\left[\rho\left(z_{1}, x_{1}\right), \varphi\left(y_{1}\right)\right]=0,
$$

de onde concluímos $\left[\rho\left(x_{1}, z_{1}\right), \varphi\left(y_{1}\right)\right]=0$, pois $\rho\left(x_{1}, z_{1}\right)=\rho\left(z_{1}, x_{1}\right)$. Portanto, $\left[\rho\left(x_{1}, z_{1}\right), \mathcal{A}_{1}\right]=0$, e como sabemos que vale (4.19), temos $\rho\left(x_{1}, z_{1}\right) \in Z(\mathcal{A})$, para todos $x_{1}, z_{1} \in \mathcal{B}_{1}$. Assim, a igualdade (4.20), nos mostra $\rho\left(x_{1}, y_{1}\right) \varphi\left(x_{0}\right)=\rho\left(x_{0} x_{1}, y_{1}\right) \in Z(\mathcal{A})$, para todos $x_{1}, y_{1} \in \mathcal{B}_{1}, x_{0} \in \mathcal{B}_{0}$. Logo, $\rho\left(\mathcal{B}_{1}, \mathcal{B}_{1}\right) \mathcal{A}_{0} \subseteq Z(\mathcal{A})$. Consequentemente, valem as seguintes igualdades

$$
\begin{aligned}
\rho\left(x_{1}, y_{1}\right)\left[a_{0}, b_{0}\right] & =\left(\rho\left(x_{1}, y_{1}\right)\right) a_{0} b_{0}-\left(\rho\left(x_{1}, y_{1}\right) b_{0}\right) a_{0} \\
& =a_{0} \rho\left(x_{1}, y_{1}\right) b_{0}-a_{0} \rho\left(x_{1}, y_{1}\right) b_{0} \\
& =0
\end{aligned}
$$

para todos $x_{1}, y_{1} \in \mathcal{B}_{1}$ e $a_{0}, b_{0} \in \mathcal{A}_{0}$

Finalmente, como $\left[\mathcal{A}_{0}, \mathcal{A}_{0}\right] \neq 0$, existem $a_{0}, b_{0} \in \mathcal{A}_{0}$ tais que $\left[a_{0}, b_{0}\right] \neq 0$. Assim, se $a \in \mathcal{A}$ e $x_{1}, y_{1} \in \mathcal{B}_{1}$, então,

$$
\rho\left(x_{1}, y_{1}\right) a\left[a_{0}, b_{0}\right]=a \rho\left(x_{1}, y_{1}\right)\left[a_{0}, b_{0}\right]=0,
$$

isto é, $\rho\left(x_{1}, y_{1}\right) \mathcal{A}\left[a_{0}, b_{0}\right]=0$, o que implica $\rho\left(x_{1}, y_{1}\right)=0$, pois $\mathcal{A}$ é uma superálgebra associativa prima. Desse modo, $\rho\left(\mathcal{B}_{1}, \mathcal{B}_{1}\right)=0$, e por hipótese, como também temos que $\varphi \mid \mathcal{B}_{0}$ é antihomomorfismo, segue $\omega\left(\mathcal{B}_{0}, \mathcal{B}_{0}\right)=0$. Logo, $\varphi$ é um superantihomomorfismo.

Encerramos o capítulo enunciando e demonstrando o resultado principal dessa seção.

Teorema 4.2.1. (Beidar, Brešar e Chebotar, [3]) Se $\varphi: \mathcal{B} \rightarrow \mathcal{A}$ um superhomomorfismo de Jordan sobrejetor da superálgebra associativa $\mathcal{B}=\mathcal{B}_{0} \oplus \mathcal{B}_{1}$ na superálgebra associativa prima $\mathcal{A}=\mathcal{A}_{0} \oplus \mathcal{A}_{1}$, onde $\mathcal{A}_{0}$ não é comutativa, então, $\varphi$ é um superhomomorfismo ou um superantihomomorfismo.

Demonstração. Pelo Lema 4.2.1, $\left.\varphi\right|_{\mathcal{B}_{0}}$ é um homomorfismo ou um antihomomorfismo. Se $\left.\varphi\right|_{\mathcal{B}_{0}}$ é um homomorfismo, concluímos que $\varphi$ é um superhomomorfismo, pelo Lema 4.2.2. Caso contrário, isto é, se $\left.\varphi\right|_{\mathcal{B}_{0}}$ é um antihomomorfismo, então, pelo Lema 4.2 .3 chegamos à conclusão que $\varphi$ é um superantihomomorfismo. 


\section{Capítulo 5}

\section{Homomorfismos de Jordan em anéis semiprimos}

Nesse capítulo vamos estudar resultados de W. E. Baxter e W. S. Martindale, $3^{\circ}$, [25], a respeito de homomorfismos de Jordan em anés semiprimos.

Inicialmente, recordemos que um homomorfismo de Jordan de um anel $T$ em um anel $R$ é uma aplicação aditiva $\varphi$ tal que $\varphi(x y+y x)=\varphi(x) \varphi(y)+\varphi(y) \varphi(x)$, para quaisquer $x, y \in T$. Do mesmo modo ao que apresentamos no capítulo anterior, estaremos assumindo que $\varphi: T \rightarrow R$ é um homomorfismo de Jordan sobrejetor e que $R$ é um anel semiprimo com característica diferente de 2. Entretanto, o resultado que apresentaremos é um tanto dissemelhante do encontrado no capítulo anterior e vamos precisar da seguinte noção.

Definição 5.0.3. Uma aplicação entre anéis $\varphi: T \rightarrow R$ é soma direta de aplicações $\sigma_{1}: T \rightarrow R$ e $\sigma_{2}: T \rightarrow R$ se existem ideais $V_{1}, V_{2}$ de $R$ tais que $V_{1} \cap V_{2}=0, \sigma_{1}(T) \subseteq V_{1}, \sigma_{2}(T) \subseteq V_{2}$ e $\varphi(a)=\sigma_{1}(a)+\sigma_{2}(a)$, para todo $a \in T$. Denotamos $\varphi=\sigma_{1} \oplus \sigma_{2}$.

O seguinte exemplo mostra uma primeira conjectura de como o resultado deveria ser no caso semiprimo.

Exemplo 5.0.5. Seja $S$ um anel primo com caraterística diferente de 2 e com involução * não trivial, isto é, ${ }^{*}$ é uma aplicação de $S$ em $S$ não nula que é aditiva e satisfaz $\left(a^{*}\right)^{*}=a$ e $(a b)^{*}=b^{*} a^{*}$. Se $R=S \oplus S$ e $\varphi: R \rightarrow R$ é tal que $\varphi(s, t)=\left(s, t^{*}\right)$, então, $R$ é semiprimo, tem característica diferente de $2, \varphi$ é aditiva e

$$
\begin{aligned}
\varphi((s, t)(m, n)+(m, n)(s, t)) & =\left(s m+m s,(t n+n t)^{*}\right) \\
& =\left(s m+m s, n^{*} t^{*}+t^{*} n^{*}\right) \\
& =\left(s, t^{*}\right)\left(m, n^{*}\right)+\left(m, n^{*}\right)\left(s, t^{*}\right)
\end{aligned}
$$

para todos $(s, t),(m, n) \in R$, isto é, $\varphi$ é um homomorfismo de Jordan. Claramente, $\varphi$ é a soma direta do homomorfismo $\sigma_{1}: R \rightarrow R$, dado por $\sigma_{1}(s, t)=(s, 0)$, com o antihomomorfismo $\sigma_{2}: R \rightarrow R$, definido por $\sigma_{2}(s, t)=\left(0, t^{*}\right)$. De fato, $V_{1}=S \oplus 0$ e $V_{2}=0 \oplus S$ são ideais de $R$ tais que $\sigma_{1}(R) \subseteq V_{1}$, $\sigma_{2}(R) \subseteq V_{2}, V_{1} \cap V_{2}=0$ e $\varphi(s, t)=\sigma_{1}(s, t)+\sigma_{2}(s, t)$.

Desse modo, este exemplo sugere a seguinte conjectura: "todo homomorfismo de Jordan sobrejetor de um anel arbitrário em um anel semiprimo de característica diferente de 2 , é soma direta de 
um homomorfismo e um antihomomorfismo". Infelizmente, esta conjectura é falsa, como pode ser visto no próximo contraexemplo, o qual foi sugerido por Kaplansky.

Exemplo 5.0.6. Seja $A$ o ideal dos elementos de termo constante nulo, da álgebra não comutativa livre gerada por $x$ e $y$ sobre um corpo $F$, e seja * a involução em $A$ determinada por $x \rightarrow x$ e $y \rightarrow y$. Se $R=A \oplus A \oplus F$ é a álgebra obtida pela adjunção de uma identidade à álgebra $A \oplus A$ pelo processo usual e $\varphi: R \rightarrow R$ é dada por $\varphi(a, b, \lambda)=\left(a, b^{*}, \lambda\right)$, para todo $(a, b, \lambda) \in R$, então, $R$ é semiprimo e $\varphi$ é um automorfismo de Jordan. De fato, $\varphi$ é uma aplicação biunívoca, aditiva e

$$
\begin{aligned}
\varphi((a, b, \lambda)(c, d, \mu)+(c, d, \mu)(a, b, \lambda)) \\
=\varphi(a c+c a+2 \lambda c+2 \mu a, b d+d b+2 \lambda d+2 \mu b, 2 \lambda \mu) \\
=\left(a c+c a+2 \lambda c+2 \mu a, d^{*} b^{*}+b^{*} d^{*}+2 \lambda d^{*}+2 \mu b^{*}, 2 \lambda \mu\right) \\
=\left(a, b^{*}, \lambda\right)\left(c, d^{*}, \mu\right)+\left(c, d^{*}, \mu\right)\left(a, b^{*}, \lambda\right)
\end{aligned}
$$

para todos $(a, b, \lambda),(c, d, \mu) \in R$.

Agora, suponha que existam um homomorfismo $\sigma_{1}: R \rightarrow R$ e um antihomomorfismo $\sigma_{2}: R \rightarrow$ $R$ tais que $\varphi=\sigma_{1}+\sigma_{2}$. O único elemento idempotente de $R$ não nulo é a identidade $(0,0,1)$. Observando que $\varphi(0,0,1)=(0,0,1), \sigma_{1}(0,0,1)$ e $\sigma_{2}(0,0,1)$ devem ser idempotentes, pois $\sigma_{1}$ é homomorfismo e $\sigma_{2}$ é antihomomorfismo, podemos concluir, sem perda de generalidade, $\sigma_{1}(0,0,1)=$ $(0,0,1)$ e $\sigma_{2}(0,0,1)=0$, o que implica $\sigma_{2}(R)=0$. Portanto, $\varphi=\sigma_{1}$ é um homomorfismo, o que claramente é uma contradição. Com efeito, por exemplo, para os elementos $(0, x, 0)$ e $(0, y, 0)$, temos $\varphi((0, x, 0)(0, y, 0))=(0, y x, 0)$, enquanto $\varphi(0, x, 0) \varphi(0, y, 0)=(0, x y, 0)$, e $y x \neq x y$.

Definição 5.0.4. Um ideal $E$ de um anel $T$ é dito essencial se $E \cap I \neq 0$, para todo ideal $I$ não nulo de $T$.

Esta definição será importante para o nosso estudo e é necessária para a seguinte conjectura: "se $\varphi$ é um homomorfismo de Jordan sobrejetor de um anel $T$ em um anel semiprimo $R$ com característica diferente de 2, então, existe um ideal essencial $E$ de $T$ tal que a restrição de $\varphi$ a $E$ é a soma direta de um homomorfismo $\sigma_{1}: E \rightarrow R$ com um antihomomorfismo $\sigma_{2}: E \rightarrow R$ "; a qual é verdadeira e com o intuito de prová-la precisaremos de alguns resultados preliminares.

Lema 5.0.4. Se $R$ é um anel semiprimo tal que $[[R, R], R]=0$, então, $R$ é comutativo.

Demonstração. Para quaisquer $x, y, z \in R$, temos $[[x, y], z]=0$, isto é, $[x, y] z=z[x, y]$. Além disso, também ocorrem as igualdades:

$$
\begin{aligned}
{[x, y]^{2} } & =[x, y] x y-[x, y] y x=[x, y x] y-y[x, y] x \\
& =y[x, y x]-y[x, y] x=y[x, y] x-y[x, y] x=0
\end{aligned}
$$

as quais implicam $[x, y] z[x, y]=[x, y]^{2} z=0$, ou seja, $[x, y] R[x, y]=0$. Portanto, como $R$ é semiprimo, ocorre $[x, y]=0$, para quaisquer $x, y \in R$, ou seja, $R$ é comutativo.

A seguir, vamos nos focar no caso em que $T$ também é semiprimo e $\varphi$ é um isomorfismo de Jordan. A demonstração da conjectura será consequência desse caso particular. Repare que como $R$ tem característica diferente de 2 e $\varphi: T \rightarrow R$ é um isomorfismo de Jordan, necessariamente $T$ tem característica diferente de 2 . 
Em virtude de $T$ ser semiprimo, já vimos na seção 4 do Capítulo 1, que existe um conjunto de ideais primos, $\left\{\mathcal{Q}_{\alpha} \mid \alpha \in \Lambda\right\}$, de $T$ tais que $\bigcap_{\alpha} \mathcal{Q}_{\alpha}=0$. Agora, definimos anéis quocientes $T_{\alpha}=T / \mathcal{Q}_{\alpha}$. Note que estes anéis são primos, pois os ideais $\mathcal{Q}_{\alpha}$ são primos. Podemos supor também, sem perda de generalidade, que os $T_{\alpha}$ 's têm característica diferente de 2. Com efeito, definimos os subconjuntos do conjunto de índices $\Lambda: \Omega=\left\{\beta \in \Lambda \mid T_{\beta}\right.$ tem característica diferente de 2$\}$ e $\Delta=$ $\left\{\gamma \in \Lambda \mid T_{\gamma}\right.$ tem característica 2$\}$. Desse modo, dados $t \in T$ e $\gamma \in \Delta$, temos $2 t+\mathcal{Q}_{\gamma}=2\left(t+\mathcal{Q}_{\gamma}\right)=0$, isto é, $2 t \in \mathcal{Q}_{\gamma}$. Assim, $2 T \subseteq \bigcap_{\gamma \in \Delta} \mathcal{Q}_{\gamma}$. Portanto, se $x \in \bigcap_{\beta \in \Omega} \mathcal{Q}_{\beta}$, então, $2 x \in \bigcap_{\alpha \in \Lambda} \mathcal{Q}_{\alpha}=0$, e necessariamente $x=0$, ou seja, $\left\{\mathcal{Q}_{\beta} \mid \beta \in \Omega\right\}$ é um conjunto de ideais primos de $T$, tais que $\bigcap_{\beta \in \Omega} \mathcal{Q}_{\beta}=0$ e $T_{\beta}$ tem característica diferente de 2 .

Lema 5.0.5. Se $I$ é o ideal de $T$ gerado por $[[T, T], T]$, então, existe um ideal $J$ de $T$ contido em $Z(T)$, tal que $I \cap J=0$ e $E=I \oplus J$ é um ideal essencial de $T$.

Demonstração. Para o conjunto de ideais $\Omega=\{K$ ideal de $T \mid I \cap K=0\}$ ordenado parcialmente pela inclusão, temos que toda cadeia $\left(K_{n}\right)_{n \in \mathbb{N}}$, onde $K_{n} \subseteq K_{n+1}$, de elementos de $\Omega$ admite a cota superior $\bigcup_{n} K_{n}$. Assim, pelo Lema de Zorn, podemos concluir que $\Omega$ possui elemento maximal $J$. Afirmamos que $E=I \oplus J$ é essencial. De fato, dado um ideal não nulo $K$ de $T$, se $I \cap K=0$, então, $(K+J) \cap I=0$, logo $J \subseteq(K+J) \in \Omega$, o que implica $J=K+J$, pois $J$ é maximal em $\Omega$. Desse modo, $K \subseteq J$ e $0 \neq K=K \cap J \subseteq K \cap E$; se $I \cap K \neq 0$, então, também $0 \neq I \cap K \subseteq E \cap K$. Como observado anteriormente, temos um conjunto de ideais primos de $T,\left\{\mathcal{Q}_{\alpha} \mid \alpha \in \Lambda\right\}$, tais que $\bigcap \mathcal{Q}_{\alpha}=0$. Portanto, valem $I J \subseteq I \cap J=0 \subseteq \mathcal{Q}_{\alpha}$, para todo $\alpha \in \Lambda$, o que implica $I \subseteq \mathcal{Q}_{\alpha}$ ou $\stackrel{\alpha}{J} \subseteq \mathcal{Q}_{\alpha}$, pois $\mathcal{Q}_{\alpha}$ é primo. Se $I \subseteq \mathcal{Q}_{\alpha}$, então, em particular, [[T $\left.\left.T_{\alpha}, T_{\alpha}\right], T_{\alpha}\right]=0$, pela definição de $I$. Logo, pelo Lema 5.0.4, temos que $T_{\alpha}$ é comutativo. Assim, $[J, T] \subseteq \mathcal{Q}_{\alpha}$. Por outro lado, se $J \subseteq \mathcal{Q}_{\alpha}$, então, $[J, T] \subseteq \mathcal{Q}_{\alpha}$, pois $\mathcal{Q}_{\alpha}$ é ideal. Em qualquer dos casos, chegamos a $[J, T] \subseteq \mathcal{Q}_{\alpha}$, o que nos permite concluir $[J, T] \subseteq \bigcap_{\alpha} \mathcal{Q}_{\alpha}=0$, isto é, $J \subseteq Z(T)$.

Prosseguindo, fixamos o ideal essencial $E$ dado pelo lema anterior. Afirmamos que, sem perda de generalidade, podemos supor que para cada $\alpha, E$ não está contido em $\mathcal{Q}_{\alpha}$, onde $\mathcal{Q}_{\alpha}$ pertence ao conjunto de ideais supracitado. De fato, defina os subconjuntos do conjunto de índices $\Lambda: \Omega=\{\beta \in$ $\left.\Lambda \mid E \nsubseteq \mathcal{Q}_{\beta}\right\}$ e $\Delta=\left\{\gamma \in \Lambda \mid E \subseteq \mathcal{Q}_{\gamma}\right\}$. Se $\bigcap_{\beta \in \Omega} \mathcal{Q}_{\beta} \neq 0$, então, $0 \neq \bigcap_{\beta \in \Omega} \mathcal{Q}_{\beta} \cap E \subseteq \bigcap_{\alpha \in \Lambda} \mathcal{Q}_{\alpha}=0$, o que é uma contradição. Portanto, vamos fixar também um conjunto de ideais primos de $T,\left\{\mathcal{Q}_{\alpha} \mid \alpha \in \Lambda\right\}$, tais que $\bigcap_{\alpha \in \Lambda} \mathcal{Q}_{\alpha}=0, T_{\alpha}$ tem característica diferente de 2 e $E \nsubseteq \mathcal{Q}_{\alpha}$, para cada $\alpha \in \Lambda$.

Dado $\alpha \in \Lambda$, considere $\tau_{\alpha}$ a projeção de $T$ sobre $T_{\alpha}$. Assim, como $\varphi: T \rightarrow R$ é um isomorfismo de Jordan, temos que $\tau_{\alpha} \varphi^{-1}$, a composta de $\tau_{\alpha}$ e $\varphi^{-1}$, é um homomorfismo de Jordan sobrejetor de $R$ em $T_{\alpha}$. Com efeito, para $a, b \in R$ arbitrários, temos

$$
\begin{aligned}
\tau_{\alpha} \varphi^{-1}(a \circ b) & =\tau_{\alpha} \varphi^{-1}\left(\varphi\left(\varphi^{-1}(a)\right) \circ \varphi\left(\varphi^{-1}(b)\right)\right) \\
& =\tau_{\alpha} \varphi^{-1}\left(\varphi\left(\varphi^{-1}(a) \circ \varphi^{-1}(b)\right)\right) \\
& =\tau_{\alpha}\left(\varphi^{-1}(a) \circ \varphi^{-1}(b)\right) \\
& =\tau_{\alpha} \varphi^{-1}(a) \circ \tau_{\alpha} \varphi^{-1}(b)
\end{aligned}
$$


o que implica que $\tau_{\alpha} \varphi^{-1}$ é um homomorfismo ou um antihomomorfismo, pelo Teorema 4.1.1, pois $T_{\alpha}$ é primo e tem característica diferente de 2. Em qualquer dos casos, obtemos que $P_{\alpha}=\operatorname{Ker}\left(\tau_{\alpha} \varphi^{-1}\right)$, o núcleo de $\tau_{\alpha} \varphi^{-1}$, é um ideal de $R$. Além disso, $P_{\alpha}$ é um ideal primo, pois se $K$ e $L$ são ideais de $R$ tais que $K L \subseteq P_{\alpha}$, então, $\tau_{\alpha} \varphi^{-1}(K L)=0$ e isso implica $\tau_{\alpha} \varphi^{-1}(K) \tau_{\alpha} \varphi^{-1}(L)=0$ ou $\tau_{\alpha} \varphi^{-1}(L) \tau_{\alpha} \varphi^{-1}(K)=0$. Portanto, como $T_{\alpha}$ é primo, concluímos $\tau_{\alpha} \varphi^{-1}(K)=0$ ou $\tau_{\alpha} \varphi^{-1}(L)=0$, isto é, $K \subseteq P_{\alpha}$ ou $L \subseteq P_{\alpha}$. Claramente $P_{\alpha}=\varphi\left(\mathcal{Q}_{\alpha}\right)$ e $E \nsubseteq \mathcal{Q}_{\alpha}$ implicam $\varphi(E) \nsubseteq P_{\alpha}$. Denotamos $R_{\alpha}=R / P_{\alpha}$ e formamos o produto direto $S=\prod_{\alpha} R_{\alpha}$. Sejam $\eta_{\alpha}: R \rightarrow R_{\alpha}$ a projeção natural e $\eta=\prod_{\alpha} \eta_{\alpha}$ o isomorfismo de $R$ em $\eta(R) \subseteq S$, isto é, $\eta(r)=\left(\eta_{\alpha}(r)\right)_{\alpha}$, para todo $r \in R$. Note que vale $\eta(r)=0$ se, e somente se, $r \in P_{\alpha}=\varphi\left(\mathcal{Q}_{\alpha}\right)$, para todo $\alpha \in \Lambda$. Assim, $\varphi^{-1}(r) \in \bigcap_{\alpha} \mathcal{Q}_{\alpha}=0$, o que implica $r=0$, isto é, $\eta$ é injetiva. Para cada $\alpha \in \Lambda, \varphi_{\alpha}=\eta_{\alpha} \varphi$, a composta de $\eta_{\alpha}$ com $\varphi$, é um homomorfismo de Jordan sobrejetor de $T$ em $R_{\alpha}$, e $R_{\alpha}$ é um anel primo de característica diferente de 2 , de onde concluímos que $\varphi_{\alpha}$ é um homomorfismo ou um antihomomorfismo, pelo Teorema 4.1.1, e restrito ao ideal $J$, ideal tal que $E=I \oplus J$ é essencial, $\varphi_{\alpha}$ é simultaneamente um homomorfismo e um antihomomorfismo, pois $J \subseteq Z(T)$. Agora, particionamos o conjunto de índices $\Lambda$ da seguinte maneira:

$$
\begin{aligned}
\Omega= & \left\{\beta \in \Lambda \mid \varphi_{\beta} \text { é um homomorfismo não nulo em } I\right\}, \\
\Delta= & \left\{\gamma \in \Lambda \mid \varphi_{\gamma} \text { é um antihomomorfismo não nulo em } I\right. \text { mas não é um } \\
& \text { homomorfismo em } I\}, \\
\Sigma= & \left\{\delta \in \Lambda \mid \varphi_{\delta} \text { é o homomorfismo nulo em } I\right\},
\end{aligned}
$$

e definimos os seguintes homomorfismos: $\epsilon_{1}: S \rightarrow S$ dado por $\epsilon_{1}\left(\left\{r_{\alpha}\right\}\right)=\left\{s_{\alpha}\right\}$, onde

$$
s_{\alpha}=\left\{\begin{array}{lll}
r_{\alpha} & \text { se } & \alpha \in \Omega \\
0 & \text { se } & \alpha \notin \Omega
\end{array}\right.
$$

$\epsilon_{2}: S \rightarrow S$ definido por $\epsilon_{2}\left(\left\{r_{\alpha}\right\}\right)=\left\{t_{\alpha}\right\}$, onde

$$
t_{\alpha}=\left\{\begin{array}{lll}
r_{\alpha} & \text { se } & \alpha \in \Delta \\
0 & \text { se } & \alpha \notin \Delta
\end{array}\right.
$$

e $\epsilon_{3}: S \rightarrow S$ tal que $\epsilon_{3}\left(\left\{r_{\alpha}\right\}\right)=\left\{v_{\alpha}\right\}$, onde

$$
v_{\alpha}=\left\{\begin{array}{lll}
r_{\alpha} & \text { se } & \alpha \in \Sigma \\
0 & \text { se } & \alpha \notin \Sigma
\end{array}\right.
$$

É fácil ver que essas aplicações são ortogonais, isto é, $\epsilon_{i} \epsilon_{j}=0$, para $i \neq j, i, j=1,2,3$, e são idempotentes, ou seja, $\epsilon_{i}^{2}=\epsilon_{i}$, para $i=1,2,3$. Além disso, como temos uma união disjunta $\Lambda=\Omega \dot{\cup} \Delta \dot{\cup} \Sigma$, segue $\epsilon_{1}+\epsilon_{2}+\epsilon_{3}=\mathrm{id}_{S}$, a aplicação identidade de $S$.

Adotando a notação $\tau_{1}=\epsilon_{1}+\epsilon_{3}$ e $\tau_{2}=\epsilon_{2}$, o seguinte resultado é válido.

Lema 5.0.6. A aplicação $\tau_{1} \eta \varphi$ é um homomorfismo de $T$ em $S$, a aplicação $\tau_{2} \eta \varphi$ é um antihomo- 
morfismo de $T$ em $S$ e $\eta \varphi=\tau_{1} \eta \varphi+\tau_{2} \eta \varphi$.

Demonstraçãa. Inicialmente, observe que temos $\eta \varphi(t)=\left\{\varphi_{\alpha}(t)\right\}_{\alpha}$, para todo $t \in T$. Desse modo, $\tau_{1} \eta \varphi(t)=\left\{\overline{\varphi_{\alpha}(t)}\right\}_{\alpha}$, onde $\overline{\varphi_{\alpha}(t)}=\varphi_{\alpha}(t)$, se $\alpha \in \Omega \cup \Sigma$; e $\overline{\varphi_{\alpha}(t)}=0$, caso contrário. Porém, para estes índices, $\varphi_{\alpha}$ é um homomorfismo de $T$ em $S$, o que implica que $\tau_{1} \eta \varphi$ é homomorfismo, pois a multiplicação é termo a termo. Analogamente, $\tau_{2} \eta \varphi(t)=\left\{\overline{\varphi_{\alpha}(t)}\right\}_{\alpha}$, onde $\overline{\varphi_{\alpha}(t)}=\varphi_{\alpha}(t)$, se $\alpha \in \Delta$; e $\overline{\varphi_{\alpha}(t)}=0$, caso contrário. Entretanto, como para estes índices, $\varphi_{\alpha}$ é um antihomomorfismo de $T$ em $S$, segue que $\tau_{2} \eta \varphi$ é um antihomomorfismo. Finalmente, podemos concluir

$$
\eta \varphi=\operatorname{id}_{S}(\eta \varphi)=\left(\epsilon_{1}+\epsilon_{2}+\epsilon_{3}\right) \eta \varphi=\tau_{1} \eta \varphi+\tau_{2} \eta \varphi
$$

Agora, definimos $\varphi_{i}=\epsilon_{i} \eta \varphi, i=1,2,3$. Neste caso, pelo Lema 5.0.6, observemos que $\varphi_{1}$ e $\varphi_{3}$ são homomorfismos de $T$ em $S$ e $\varphi_{2}$ é um antihomomorfismo de $T$ em $S$.

Lema 5.0.7. A inclusão $\varphi_{i}([T, T]) \subseteq \eta(R)$ é válida, para quaisquer $i=1,2,3$.

Demonstração. Seja $\psi=\eta \varphi$. Dados $x, y \in T$, temos

$$
\begin{aligned}
\psi(x) \psi(y) & =\left(\epsilon_{1}+\epsilon_{2}+\epsilon_{3}\right)(\psi(x))\left(\epsilon_{1}+\epsilon_{2}+\epsilon_{3}\right)(\psi(y)) \\
& =\left(\varphi_{1}(x)+\varphi_{2}(x)+\varphi_{3}(x)\right)\left(\varphi_{1}(y)+\varphi_{2}(y)+\varphi_{3}(y)\right) \\
& =\varphi_{1}(x) \varphi_{1}(y)+\varphi_{2}(x) \varphi_{2}(y)+\varphi_{3}(x) \varphi_{3}(y)
\end{aligned}
$$

pois $\varphi_{i}(x) \varphi_{j}(y)=0$, para $i \neq j$. De fato, sem perda de generalidade, por exemplo para $i=1 \mathrm{e}$ $j=2$, enquanto $\varphi_{i}(x)$ tem todas as entradas com índices pertencentes a $\Delta \dot{U} \Sigma$ nulas, $\varphi_{2}(y)$ possui as entradas com índices pertencentes a $\Omega \dot{\cup} \Sigma$ nulas. Por outro lado, também valem

$$
\begin{aligned}
\psi(x y) & =\varphi_{1}(x y)+\varphi_{2}(x y)+\varphi_{3}(x y) \\
& =\varphi_{1}(x) \varphi_{1}(y)+\varphi_{2}(x) \varphi_{2}(y)+\varphi_{3}(x) \varphi_{3}(y) .
\end{aligned}
$$

Pelos dois últimos blocos de igualdades, obtemos

$$
\psi(x y)-\psi(x) \psi(y)=\varphi_{2}(x y-y x)
$$

o que mostra que $\varphi_{2}([T, T]) \subseteq \eta(R)$ é verificada, pois $\psi(x y)-\psi(x) \psi(y)=\eta(\varphi(x y)-\varphi(x) \varphi(y))$. Analogamente, temos $\psi(x) \psi(y)=\varphi_{1}(x) \varphi_{1}(y)+\varphi_{2}(x) \varphi_{2}(y)+\varphi_{3}(x) \varphi_{3}(y) \mathrm{e}$

$$
\psi(y x)=\varphi_{1}(y) \varphi_{1}(x)+\varphi_{2}(x) \varphi_{2}(y)+\varphi_{3}(y) \varphi_{3}(x)
$$

o que implica

$$
\varphi_{1}(x y-y x)+\varphi_{3}(x y-y x)=\psi(x) \psi(y)-\psi(y x),
$$

isto é, $\varphi_{1}([x, y])+\varphi_{3}([x, y]) \in \eta(R)$.

Agora, afirmamos que vale $\varphi_{3}([T, T])=0$. Com efeito, sabemos que para $\delta \in \Sigma$, $\varphi_{\delta}$ é o homomorfismo nulo em $I$, então, se $x, y, z \in T, \varphi_{\delta}([[x, y], z])=0$ implica $\varphi_{\delta}([x, y]) \varphi_{\delta}(z)=\varphi_{\delta}(z) \varphi_{\delta}([x, y])$. 
Desse modo, $\varphi_{\delta}([x, y]) \varphi_{\delta}(z) \varphi_{\delta}([x, y])=\varphi_{\delta}\left([x, y]^{2}\right) \varphi_{\delta}(z)$. Além disso, temos

$$
\begin{aligned}
{[x, y]^{2} } & =[x, y] x y-[x, y] y x \\
& =[x, y] x y-x y[x, y]+x y[x, y]-[x, y] y x \\
& =[[x, y], x y]+x[y x, y]-[y x, y] x+[y x, y] x-[x y, y] x \\
& =[[x, y], x y]-[[y x, y], x]+[[y, x], y] x \in I .
\end{aligned}
$$

Portanto, $\varphi_{\delta}\left([x, y]^{2}\right)=0$; e como $\varphi_{\delta}$ é sobrejetora, chegamos a $\varphi_{\delta}([x, y]) R_{\delta} \varphi_{\delta}([x, y])=0$. Logo, $\varphi_{\delta}([x, y])=0$, pois $R_{\delta}$ é um anel primo; e podemos concluir $\varphi_{1}([T, T]) \subseteq \eta(R)$ e também $\varphi_{3}([T, T])=0 \subseteq \eta(R)$.

Lema 5.0.8. Para $i=1,2,3$, temos $\varphi_{i}(I) \subseteq \eta(R)$.

Demonstração. Dados $a \in[T, T]$ e $b \in T$, decorrem do Lema 5.0.7 as igualdades:

$$
\begin{aligned}
\varphi_{1}(a) \varphi_{1}([a, b]) & =\varphi_{1}(a)\left(\varphi_{1}(a) \varphi_{1}(b)-\varphi_{1}(b) \varphi_{1}(a)\right) \\
& =\varphi_{1}(a) \varphi_{1}(a b)-\varphi_{1}(a b) \varphi_{1}(a) \\
& =\varphi_{1}([a, a b]) \in \eta(R)
\end{aligned}
$$

isto é, $\varphi_{1}(a) \varphi_{1}([a, b])=\varphi_{1}([a, a b]) \in \eta(R)$, cuja linearização em $a$ é dada por

$$
\varphi_{1}(a) \varphi_{1}([x, b])+\varphi_{1}(x) \varphi_{1}([a, b]) \in \eta(R) .
$$

Substituindo $a$ por $[c, d]$, onde $c, d \in T$, obtemos

$$
\varphi_{1}([c, d]) \varphi_{1}([x, b])+\varphi_{1}(x) \varphi_{1}([[c, d], b]) \in \eta(R)
$$

o que implica $\varphi_{1}(x) \varphi_{1}([[c, d], b]) \in \eta(R)$, pois $\varphi_{1}([c, d]) \varphi_{1}([x, b]) \in \eta(R)$, pelo Lema 5.0.7, isto é,

$$
\varphi_{1}\{x[[c, d], b]\} \in \eta(R) .
$$

Do mesmo modo, temos $\varphi_{1}([a, b]) \varphi_{1}(a)=\varphi_{1}([a, b a]) \in \eta(R)$, cuja linearização em $a$ resulta em

$$
\varphi_{1}([a, b]) \varphi_{1}(x)+\varphi_{1}([x, b]) \varphi_{1}(a) \in \eta(R),
$$

Escolhendo $a=[c, d]$, obtemos

$$
\varphi_{1}([[c, d], b]) \varphi_{1}(x)+\varphi_{1}([x, b]) \varphi_{1}([c, d]) \in \eta(R) .
$$

Assim, como $\varphi_{1}([x, b]) \varphi_{1}([c, d]) \in \eta(R)$, podemos concluir

$$
\varphi_{1}\{[[c, d], b] x\} \in \eta(R) .
$$

Agora, por (5.1) e (5.2), seguem as igualdades

$$
\begin{aligned}
\varphi_{1}\{y[[c, d], b] x\} & =\varphi_{1}\{y[[c, d], b] x-x y[[c, d], b]+x y[[c, d], b]\} \\
& =\varphi_{1}\{[y[[c, d], b], x]\}+\varphi_{1}\{x y[[c, d], b]\} \in \eta(R) .
\end{aligned}
$$


Portanto $\varphi_{1}(I) \subseteq \eta(R)$. Prosseguindo, vamos repetir o mesmo raciocínio para $\varphi_{2}$. Dados $a \in$ $[T, T]$ e $b \in T$, temos

$$
\varphi_{2}(a) \varphi_{2}([a, b])=\varphi_{2}([a, b a]) \in \eta(R),
$$

cuja linearização em $a$, resulta em

$$
\varphi_{2}(a) \varphi_{2}([x, b])+\varphi_{2}(x) \varphi_{2}([a, b]) \in \eta(R) .
$$

Substituindo $a$ por $[c, d], c, d \in T$, obtemos

$$
\varphi_{2}([c, d]) \varphi_{2}([x, b])+\varphi_{2}(x) \varphi_{2}([[c, d], b]) \in \eta(R) .
$$

Assim, como $\varphi_{2}([c, d]) \varphi_{2}([x, b]) \in \eta(R)$, pelo Lema 5.0.7, segue

$$
\varphi_{2}(x) \varphi_{2}([[c, d], b]) \in \eta(R)
$$

o que implica

$$
\varphi_{2}\{[[c, d], b] x\} \in \eta(R) .
$$

Analogamente, temos $\varphi_{2}([a, b]) \varphi_{2}(a)=\varphi_{2}([a, a b]) \in \eta(R)$, que linearizando em $a$ nos fornece $\varphi_{2}([a, b]) \varphi_{2}(x)+\varphi_{2}([x, b]) \varphi_{2}(a) \in \eta(R)$. Desse modo, tomando $a=[c, d]$, obtemos

$$
\varphi_{2}([[c, d], b]) \varphi_{2}(x)+\varphi_{2}([x, b]) \varphi_{2}([c, d]) \in \eta(R),
$$

de onde concluímos

$$
\varphi_{2}\{x[[c, d], b]\} \in \eta(R),
$$

pois $\varphi_{2}([x, b]) \varphi_{2}([c, d]) \in \eta(R)$. Portanto, de (5.3) e (5.4), seguem

$$
\begin{aligned}
\varphi_{2}\{x[[c, d], b] y\} & =\varphi_{2}\{x[[c, d], b] y-y x[[c, d], b]+y x[[c, d], b]\} \\
& =\varphi_{2}\{[x[[c, d], b], y]\}+\varphi_{2}\{y x[[c, d], b]\} \in \eta(R) .
\end{aligned}
$$

Logo, $\varphi_{2}(I) \subseteq \eta(R)$. Finalmente, para $\varphi_{3}$ basta observarmos, assim como anteriormente, que vale $\varphi_{3}(I)=0 \subseteq \eta(R)$.

Lema 5.0.9. As igualdades $\varphi_{1}(J)=\varphi_{2}(J)=0$ e $\varphi_{3}(J) \subseteq \eta(R)$ são válidas.

Demonstração. Suponhamos que ocorre $\varphi_{1}(J) \neq 0$. Note que, como $\varphi_{1}=\epsilon_{1} \eta \varphi$, onde $\epsilon_{1}$ é a "projeção" nas coordenadas cujos índices pertencem a $\Omega$, deve existir $\beta \in \Omega$ tal que $\varphi_{\beta}(J) \neq 0$. Desse modo, pela definição de $\Omega$, sabemos que vale $\varphi_{\beta}(I) \neq 0$. Todavia,

$$
\varphi_{\beta}(I) \varphi_{\beta}(J)=\varphi_{\beta}(I J) \subseteq \varphi_{\beta}(I \cap J)=0,
$$

o que é uma contradição, pois $R_{\beta}$ é um anel primo. Pelo mesmo argumento, supondo $\varphi_{2}(J) \neq 0$, como $\varphi_{2}=\epsilon_{2} \eta \varphi$, onde $\epsilon_{2}$ é a "projeção" nas coordenadas cujos índices pertencem a $\Delta$, deve existir $\gamma \in \Delta$ tal que $\varphi_{\gamma}(J) \neq 0$. Assim, pela definição de $\Delta$, sabemos que ocorre $\varphi_{\gamma}(I) \neq 0$. Entretanto,

$$
\varphi_{\gamma}(I) \varphi_{\gamma}(J)=\varphi_{\gamma}(J I) \subseteq \varphi_{\beta}(J \cap I)=0,
$$


o que novamente é uma contradição por $R_{\gamma}$ ser um anel primo. Em consequência desses fatos, temos

$$
\varphi_{3}(J)=\eta \varphi(J)-\varphi_{1}(J)-\varphi_{2}(J)=\eta \varphi(J) \subseteq \eta(R) .
$$

Já estamos em condição de enunciar e provar os resultados principais desse capítulo.

Teorema 5.0.2. (Baxter e Martindale, 3rd, [25]) Se $\varphi$ é um isomorfismo de Jordan de um anel semiprimo $T$ em um anel semiprimo $R$ com característica diferente de 2 , então, existe um ideal essencial $E$ de $T$ tal que a restrição de $\varphi$ a $E$ é uma soma direta $\sigma_{1} \oplus \sigma_{2}$, onde $\sigma_{1}$ é um homomorfismo de $E$ em $R$ e $\sigma_{2}$ é um antihomomorfismo de $E$ em $R$.

Demonstração. Seja $E$ o ideal essencial dado pelo Lema 5.0.5. Recordemos que tínhamos fixado as notações: $\tau_{1} \eta \varphi=\left(\epsilon_{1}+\epsilon_{3}\right) \eta \varphi=\varphi_{1}+\varphi_{3}$ e $\tau_{2} \eta \varphi=\epsilon_{2} \eta \varphi=\varphi_{2}$. Assim, pelos Lemas 5.0.8 e 5.0.9, temos $\tau_{i} \eta \varphi(E) \subseteq \eta(R)$, onde $i=1,2$. Afirmamos que $V_{1}=\tau_{1} \eta \varphi(E)$ e $V_{2}=\tau_{2} \eta \varphi(E)$ são ideais de $\eta(R)$ tais que $V_{1} \cap V_{2}=0$. Com efeito, para $\tau_{1} \eta \varphi(u) \in V_{1}$ e $\eta \varphi(t) \in \eta(R)$, temos

$$
\begin{gathered}
\tau_{1} \eta \varphi(u) \eta \varphi(t)=\tau_{1} \eta \varphi(u)\left(\tau_{1} \eta \varphi(t)+\tau_{2} \eta \varphi(t)\right)=\tau_{1} \eta \varphi(u t) \in V_{1} \mathrm{e} \\
\eta \varphi(t) \tau_{1} \eta \varphi(u)=\left(\tau_{1} \eta \varphi(t)+\tau_{2} \eta \varphi(t)\right) \tau_{1} \eta \varphi(u)=\tau_{1} \eta \varphi(t u) \in V_{1} .
\end{gathered}
$$

Do mesmo modo, para $\tau_{2} \eta \varphi(u) \in V_{2}$ e $\eta \varphi(t) \in \eta(R)$, temos

$$
\begin{gathered}
\tau_{2} \eta \varphi(u) \eta \varphi(t)=\tau_{2} \eta \varphi(u)\left(\tau_{1} \eta \varphi(t)+\tau_{2} \eta \varphi(t)\right)=\tau_{2} \eta \varphi(t u) \in V_{2} \mathrm{e} \\
\eta \varphi(t) \tau_{2} \eta \varphi(u)=\left(\tau_{1} \eta \varphi(t)+\tau_{2} \eta \varphi(t)\right) \tau_{2} \eta \varphi(u)=\tau_{2} \eta \varphi(u t) \in V_{2} .
\end{gathered}
$$

Além disso, os elementos de $V_{1}$ possuem as coordenadas com índices pertencentes a $\Delta$ todas nulas, e os elementos de $V_{2}$ possuem as coordenadas cujos índices pertencem a $\Omega \dot{\cup} \Sigma$ todas nulas, logo $V_{1} \cap V_{2}=0$. Desse modo, temos que $\left.\eta \varphi\right|_{E}$ é a soma direta do homomorfismo $\left.\tau_{1} \eta \varphi\right|_{E}$ com o antihomomorfismo $\left.\tau_{2} \eta \varphi\right|_{E}$. Finalmente, aplicando o isomorfismo $\eta^{-1}: \eta(R) \rightarrow R$ a $\left.\tau_{1} \eta \varphi\right|_{E}$ e $\left.\tau_{2} \eta \varphi\right|_{E}$, obtemos o resultado desejado para $\sigma_{1}=\left.\eta^{-1} \tau_{1} \eta \varphi\right|_{E}$ e $\sigma_{2}=\left.\eta^{-1} \tau_{2} \eta \varphi\right|_{E}$.

Finalmente, a seguir, apresentamos a demonstração do caso mais geral.

Teorema 5.0.3. (Baxter e Martindale, 3rd, [25]) Se $\varphi$ é um homomorfismo de Jordan sobrejetor de um anel $T$ em um anel semiprimo $R$ que possui característica diferente de 2 , então, existe um ideal essencial $E$ de $T$ tal que a restrição de $\varphi$ a $E$ é uma soma direta $\sigma_{1} \oplus \sigma_{2}$, onde $\sigma_{1}$ é um homomorfismo de $E$ em $R$ e $\sigma_{2}$ é um antihomomorfismo de $E$ em $R$.

Demonstração. Inicialmente, observe que $K=\operatorname{Ker}(\varphi)$, o núcleo de $\varphi$, é um ideal de $T$. De fato, existem ideais primos $\left\{P_{\alpha} \mid \alpha \in \Lambda\right\}$ de $R$ tais que $\bigcap_{\alpha} P_{\alpha}=0$. Relembrando que $\eta_{\alpha}$ é a projeção natural de $R$ sobre $R_{\alpha}=R / P_{\alpha}$ e $\varphi_{\alpha}=\eta_{\alpha} \varphi$ é um homomorfismo de Jordan sobrejetor do anel $T$ no anel primo $R_{\alpha}$, temos que $\varphi_{\alpha}$ é um homomorfismo ou antihomomorfismo. Dados $a \in K$ e $x \in T$, ocorre $\varphi_{\alpha}(a x)=\varphi_{\alpha}(a) \varphi_{\alpha}(x)$ ou $\varphi_{\alpha}(a x)=\varphi_{\alpha}(x) \varphi_{\alpha}(a)$. Em qualquer uma das alternativas, $\varphi_{\alpha}(a)=\eta_{\alpha} \varphi(a)=0$ implica $\varphi_{\alpha}(a x)=0$, isto é, $\eta_{\alpha} \varphi(a x)=0$. Portanto, $\varphi(a x) \in P_{\alpha}$, para todo 
$\alpha \in \Lambda$, ou seja, $\varphi(a x) \in \bigcap_{\alpha} P_{\alpha}=0$. Logo, $a x \in K$. Analogamente, $x a \in K$ e podemos concluir que $K$ é um ideal.

Desse modo, podemos definir $\bar{T}=T / K$ e $\bar{\varphi}: \bar{T} \rightarrow R$ por $\bar{\varphi}(\bar{x})=\varphi(x)$, para todo $\bar{x} \in \bar{T}$. De fato, $\bar{\varphi}$ está bem definida e é um isomorfismo de Jordan de $\bar{T}$ em $R$. Agora, suponha que $a \in T$ é tal que $\bar{a} \bar{T} \bar{a}=0$. Assim, temos

$$
0=\bar{\varphi}(\bar{a} \bar{t} \bar{a})=\varphi(a) \varphi(t) \varphi(a)
$$

para todo $t \in T$. Portanto, como $\varphi$ é sobrejetora, segue $\varphi(a) R \varphi(a)=0$, o que implica $\varphi(a)=0$, pois $R$ é um anel semiprimo, ou seja, $\bar{a}=0$. Logo, $\bar{T}$ é semiprimo. Note que as condições do Teorema 5.0.2 são satisfeitas, de onde concluímos que existe um ideal essencial $\bar{E}$ de $\bar{T}$ tal que que $\left.\bar{\varphi}\right|_{\bar{E}}$ é uma soma direta $\bar{\sigma}_{1} \oplus \bar{\sigma}_{2}$, onde $\bar{\sigma}_{1}$ é um homomorfismo de $\bar{E}$ em $R$ e $\bar{\sigma}_{2}$ é um antihomomorfismo de $\bar{E}$ em $R$.

Agora, definimos $F=\rho^{-1}(\bar{E})$, onde $\rho$ é a projeção natural de $T$ sobre $\bar{T}$. Afirmamos que $F$ é um ideal essencial de $T$. Claramente, $K=\rho^{-1}(0) \subseteq \rho^{-1}(\bar{E})=F$. Seja $V$ um ideal não nulo de $T$. Se $V \subseteq K$, então, $0 \neq V=V \cap K \subseteq V \cap F$; e se $V \nsubseteq K$, então, podemos escolher $v \in V$ tal que $v \notin K$ e $\bar{v} \in \bar{E}$. De fato, se $V \nsubseteq K$, então, $K$ está contido propriamente em $V+K$, e logo existe $0 \neq \bar{x} \in \overline{V+K}$, o que significa $x=v+k, \operatorname{com} v \in V$ e $k \in K$ e $v$ é um tal elemento como queremos. Consequentemente, $v=u+k$, onde $u \in F$ e $k \in K \subseteq F$. Portanto, $0 \neq v \in V \cap F$. Finalmente, para $i=1,2$, definindo $\sigma_{i}: F \rightarrow R$ por $\sigma_{i}(u)=\bar{\sigma}_{i}(\bar{u})$, para todo $u \in F$, temos que $\sigma_{1}$ é um homomorfismo tal que $\sigma_{1}(F)=\bar{\sigma}_{1}(\bar{E}) \subseteq V_{1}$ e $\sigma_{2}$ é um antihomomorfismo tal que $\sigma_{2}(F)=\bar{\sigma}_{2}(\bar{E}) \subseteq V_{2}$, onde $V_{1}$ e $V_{2}$ são ideais de $R$ tais que $V_{1} \cap V_{2}=0$, e $\left.\varphi\right|_{F}=\sigma_{1} \oplus \sigma_{2}$. 


\section{Capítulo 6}

\section{Identidades funcionais e superhomomorfismos de Jordan}

Nesse capítulo introduzimos em linhas gerais os conceitos básicos da teoria de identidades funcionais referentes a Matej Brešar, Mikhail Chebotar e Wallace Martindale $3^{\circ}$ em [8], apresentamos a generalização dessa teoria para o contexto de superálgebras dada por Yu Wang [32], uma aplicação para a classificação dos superhomomorfismos de Jordan referente a Yao Wang e Yu Wang em [33] e uma contribuição para a classificação das superderivações de Jordan de grau 0.

\subsection{A teoria de identidades funcionais em anéis}

Ao invés de iniciarmos com uma definição rigorosa de identidade funcional, vamos primeiramente ilustrá-la com dois exemplos simples.

Exemplo 6.1.1. Sejam $A$ um anel e $E, F: A \rightarrow A$ aplicações tais que

$$
E(x) y+F(y) x=0 \quad \text { para todos } x, y \in A .
$$

Observe que não assumimos quaisquer condições a mais sobre as funções $E$ e $F$, além de serem aplicações entre conjuntos que satisfazem (6.1). Uma possibilidade trivial é $E=F=0$. Se, por exemplo, $A$ é comutativo, podemos tomar $E$ como sendo a identidade e $F=-E$. De modo mais geral, suponha que $A$ contém um ideal central $I$, isto é, $I \subseteq Z(A)$. Dado qualquer $c \in I$, podemos definir $E$ e $F$ por $E(x)=-F(x)=c x$, e (6.1) é satisfeita. Agora, se $A$ é um anel primo associativo e não comutativo, note que (6.1) implica

$$
(E(x) y z) w=-F(y z) x w=(E(x w) y) z=-(F(y) x) w z=E(x) y w z,
$$

para todos $x, y, z, w \in A$. Portanto, temos $E(A) A[A, A]=0$. Desse modo, $E=0$ e consequentemente, $F=0$.

Suponha que $A$ é semiprimo e $E \neq 0$. Seja $I=$ ideal $\langle E(A)\rangle$, isto é, o ideal gerado pela imagem de $E$. Pelos mesmos cálculos anteriores, concluímos $[I, A] A[I, A]=0$, o que implica $[I, A]=0$, ou seja, $I$ é um ideal central. Finalmente, suponha $A=M_{n}(C), n \geq 1$, onde $C$ é um anel comutativo com elemento identidade. Se $n \geq 2$, então, veremos que ideal $\langle[A, A]\rangle$ contém a matriz identidade. 
Para isso, temos que se $i \neq j$, então,

$$
\begin{gathered}
\text { ideal }\langle[A, A]\rangle \ni\left[e_{i j}, e_{j i}\right]=\left(e_{i j} e_{j i}-e_{j i} e_{i j}\right)=e_{i i}-e_{j j} \mathrm{e} \\
\text { ideal }\langle[A, A]\rangle \ni\left[e_{i i}, e_{i j}\right]=\left(e_{i i} e_{i j}-e_{i j} e_{i i}\right)=e_{i j},
\end{gathered}
$$

onde as $e_{i j}$ 's são matrizes elementares, isto é, cuja entrada $(i, j)$ é igual a 1 e todas as restantes são nulas. Assim, por exemplo, para $n=4$, temos que a matriz

$$
\left(e_{11}-e_{22}\right)+\left(e_{33}-e_{44}\right)=\left[\begin{array}{cccc}
1 & 0 & 0 & 0 \\
0 & -1 & 0 & 0 \\
0 & 0 & 1 & 0 \\
0 & 0 & 0 & -1
\end{array}\right]
$$

pertence a $I$, e portanto o seu quadrado, que é igual à matriz identidade, também pertence. Pelo mesmo raciocínio, podemos concluir este fato para qualquer $n$ par. Se, por exemplo, $n=3$, então, a matriz

$$
e_{11}-e_{22}=\left[\begin{array}{ccc}
1 & 0 & 0 \\
0 & -1 & 0 \\
0 & 0 & 0
\end{array}\right]
$$

pertence ao ideal. Consequentemente, a matriz

$$
\left[\begin{array}{lll}
1 & 0 & 0 \\
0 & 1 & 0 \\
1 & 0 & 1
\end{array}\right]\left[\begin{array}{ccc}
1 & 0 & 0 \\
0 & -1 & 0 \\
0 & 0 & 0
\end{array}\right]\left[\begin{array}{ccc}
1 & 0 & 1 \\
0 & -1 & 0 \\
0 & 0 & 1
\end{array}\right]=\left[\begin{array}{lll}
1 & 0 & 1 \\
0 & 1 & 0 \\
1 & 0 & 1
\end{array}\right]
$$

também pertence a $I$ (trocamos a terceira coluna da matriz $e_{11}-e_{22}$ pela terceira somada com a primeira, e depois trocamos a terceira linha da nova matriz pela terceira somada com a primeira). Além disso, como $e_{13}$ e $e_{31}$ estão em $I$, segue que

$$
\left[\begin{array}{lll}
1 & 0 & 1 \\
0 & 1 & 0 \\
1 & 0 & 1
\end{array}\right]-\left[\begin{array}{lll}
0 & 0 & 1 \\
0 & 0 & 0 \\
0 & 0 & 0
\end{array}\right]-\left[\begin{array}{lll}
0 & 0 & 0 \\
0 & 0 & 0 \\
1 & 0 & 0
\end{array}\right]=\left[\begin{array}{lll}
1 & 0 & 0 \\
0 & 1 & 0 \\
0 & 0 & 1
\end{array}\right]
$$

pertence a $I$, como era desejado. Pelo mesmo raciocínio, podemos concluir o resultado para qualquer $n$ ímpar. Desse modo, temos $E=0$ e, nesse caso, também $F=0$.

Dizemos que as funções $E$ e $F$ que satisfazem a identidade funcional ou a I.F. (6.1) são suas soluções, e $E=F=0$ é a solução padrão desta identidade funcional. Podemos sintetizar as observações anteriores da seguinte maneira: "se $A$ é um anel semiprimo (respectivamente, primo), então, existe uma solução não padrão de (6.1) se, e somente se, $A$ contém um ideal central não nulo; além disso, se $A=M_{n}(C)$, onde $C$ é um anel comutativo com elemento identidade, então, existe uma solução não padrão de (6.1) se, e somente se, $n=1 . "$

A seguir, vamos considerar um exemplo um pouco mais geral do que o anterior. 
Exemplo 6.1.2. Sejam $A$ um anel e $E, F: A \rightarrow A$ aplicações tais que

$$
E(x) y+F(y) x \in Z(A), \quad \text { para todos } x, y \in A
$$

Observe que podemos reescrever (6.2), incluindo mais uma variável $z \in A$, do seguinte modo: $[E(x) y+F(y) x, z]=0$, para todos $x, y, z \in A$. Assim, como no exemplo anterior, definimos $E=F=$ 0 como sendo a solução padrão de (6.2). Contudo, soluções não padrão de (6.2) existem não somente em anéis comutativos, mas também no anel $A=M_{2}(C)$, onde $C$ é anel comutativo com identidade. De fato, defina $E, F: A \rightarrow A$ por $E(x)=F(x)=x-\operatorname{tr}(x) I_{2}$, para todo $x \in A$, onde $\operatorname{tr}(x)$ é o traço da matriz $x$ e $I_{2}$ denota a matriz identidade de ordem 2. Desse modo, se $x=\left(\begin{array}{ll}x_{1} & x_{2} \\ x_{3} & x_{4}\end{array}\right)$ e $y=\left(\begin{array}{ll}y_{1} & y_{2} \\ y_{3} & y_{4}\end{array}\right)$, temos $E(x) y+F(y) x=\alpha I_{2}$, onde $\alpha=-x_{1} y_{4}-x_{4} y_{1}+x_{2} y_{3}+x_{3} y_{2}$, o que implica $E(x) y+F(y) x \in Z(A)$. Por outro lado, se $A=M_{n}(C)$, com $n \geq 3$, então, (6.2) possui apenas a solução padrão. De fato, fixe $\pi(x, y)=E(x) y+F(y) x$, para $x, y \in A$. Assuma $\pi(x, y) \in Z(A)$, para todos $x, y \in A$. Portanto, para quaisquer $x, y, t \in A$, o elemento $\pi(x t, y)-\pi(x, y) t=E(x t) y-E(x) y t$ comuta com $t$, isto é, $(E(x t) y-E(x) y t) t-t(E(x t) y-E(x) y t)=0$, o que implica

$$
-E(x) y t^{2}+(t E(x)+E(x t)) y t-t E(x t) y=0 .
$$

Prosseguindo, consideremos novamente as matrizes elementares $e_{i j}$ 's $\in A$. Seja $1 \leq i \leq n$ e fixe $t=e_{12}+e_{23}$ e $y=e_{i 1}$. Substituindo esses valores na última relação e usando que vale

$$
e_{i j} e_{k l}=\left\{\begin{array}{cl}
e_{i l}, & \text { se } j=k \\
0, & \text { caso contrário, }
\end{array}\right.
$$

obtemos $E(x) e_{i i}=0$, ou seja, para cada $i$, a $i$-ésima coluna de $E(x)$ é nula. Logo, $E(x)=0$, o que implica $E=0$ e consequentemente, também $F=0$.

Em suma, podemos afirmar: "se $A=M_{n}(C)$, onde $C$ é um anel comutativo com elemento identidade, então, existe uma solução não padrão de (6.2) se, e somente se, $n \leq 2$."

Finalmente, vamos definir o conceito de identidade funcional.

Definição 6.1.1. Sejam $A$ um anel, $R$ um subconjunto não vazio de $A$ e $F_{i}: R^{m} \rightarrow A$ funções, onde $R^{m}$ é o produto cartesiano de $m$ cópias de $R$ e $1 \leq i \leq n$. Além disso, considere $X=$ $\left\{x_{1}, y_{1}, x_{2}, y_{2}, \ldots\right\}$ um conjunto enumerável e $\mathbb{Z}\langle X\rangle$ a álgebra livre gerada por $X$ sobre $\mathbb{Z}$. Seja $f=f\left(x_{1}, x_{2}, \ldots, x_{m}, y_{1}, y_{2}, \ldots, y_{n}\right) \in \mathbb{Z}\langle X\rangle$, com $m \geq 1$ e $n \geq 0$, um polinômio tal que pelo menos um de seus monômios de maior grau tem coeficiente 1. Dizemos que $f$ é uma identidade funcional em $R$ com funções $F_{1}, \ldots, F_{n}$ se

$$
f\left(r_{1}, \ldots, r_{m}, F_{1}\left(r_{1}, \ldots, r_{m}\right), \ldots, F_{n}\left(r_{1}, \ldots, r_{m}\right)\right)=0
$$

para todos $r_{1}, \ldots, r_{m} \in R$. Neste caso, dizemos que $F_{1}, \ldots, F_{n}$ são soluções desta identidade funcional.

A fim de ilustrar esta definição, considere por exemplo a seguinte identidade funcional:

$$
E(x, y) z+F(x, z) y+G(y, z) x \in Z(A), \quad \text { para todos } x, y, z \in A .
$$


Inicialmente, note que podemos reescrevê-la como

$$
[E(x, y) z+F(x, z) y+G(y, z) x, u]=0, \quad \text { para todos } x, y, z, u \in A .
$$

Agora, escolhemos o polinômio $f$ como sendo

$$
f\left(x_{1}, x_{2}, x_{3}, x_{4}, y_{1}, y_{2}, y_{3}\right)=\left[y_{1} x_{1}+y_{2} x_{2}+y_{3} x_{3}, x_{4}\right]
$$

$m=4, n=3$ e $R=A$. As funções $F_{i}: A^{4} \rightarrow A, 1 \leq i \leq 3$, são definidas de modo que $F_{i}$ não dependa da $i$-ésima variável, como por exemplo $F_{2}\left(x_{1}, x_{2}, x_{3}, x_{4}\right)=F\left(x_{1}, x_{3}\right)$.

Na sequência, vamos introduzir um tipo mais fundamental de identidades funcionais, o qual utilizaremos no decorrer do capítulo, e que corresponde ao polinômio $\sum_{i} y_{1 i} x_{i}+\sum_{j} x_{j} y_{2 j}$. Para descrevê-las melhor, necessitaremos fixar algumas notações. Considere $m \in \mathbb{N}$ fixo. Para os elementos $x_{1}, x_{2}, \ldots, x_{m}$ do anel $A$, escrevemos

$$
\bar{x}_{m}=\left(x_{1}, \ldots, x_{m}\right) \in A^{m} .
$$

Por conveniência, definimos $A^{0}=\{0\}$. Além disso, para $1<i<m$, fixamos

$$
\bar{x}_{m}^{i}=\left(x_{1}, \ldots, x_{i-1}, x_{i+1}, \ldots, x_{m}\right) \in A^{m-1} .
$$

Observe que naturalmente $\bar{x}_{m}^{1}=\left(x_{2}, \ldots, x_{m}\right)$ e $\bar{x}_{m}^{m}=\left(x_{1}, \ldots, x_{m-1}\right)$. Analogamente, para $1 \leq i<j \leq m$, fixamos

$$
\bar{x}_{m}^{i j}=\bar{x}_{m}^{j i}=\left(x_{1}, \ldots, x_{i-1}, x_{i+1}, \ldots, x_{j-1}, x_{j+1}, \ldots, x_{m}\right) \in A^{m-2} .
$$

Novamente para $i$ e $j$ iguais a 1 ou $m$, essa notação tem um significado análogo ao da anterior: "retiramos" da $m$-upla as variáveis $i$-ésima e $j$-ésima. Em particular, temos $\bar{x}_{1}^{1}=\bar{x}_{2}^{12}=0$.

Sejam $\mathcal{I}$ e $\mathcal{J}$ subconjuntos finitos de $\mathbb{N}$ e $m \in \mathbb{N}$ tais que $\mathcal{I}, \mathcal{J} \subseteq\{1,2, \ldots, m\}$. Considere $E_{i}, F_{j}: A^{m-1} \rightarrow A, i \in \mathcal{I}, j \in \mathcal{J}$, aplicações arbitrárias. Vale ressaltar que uma aplicação definida em $A^{0}=\{0\}$ será considerada como um elemento fixo de $A$. Estamos interessados em identidades funcionais envolvendo as seguintes expressões:

$$
\begin{aligned}
& \sum_{i \in \mathcal{I}} E_{i}\left(\bar{x}_{m}^{i}\right) x_{i}=\sum_{i \in \mathcal{I}} E_{i}\left(x_{1}, \ldots, x_{i-1}, x_{i+1}, \ldots, x_{m}\right) x_{i} \mathrm{e} \\
& \sum_{j \in \mathcal{J}} x_{j} F_{j}\left(\bar{x}_{m}^{j}\right)=\sum_{j \in \mathcal{J}} x_{j} F_{j}\left(x_{1}, \ldots, x_{j-1}, x_{j+1}, \ldots, x_{m}\right) .
\end{aligned}
$$

Mais precisamente, as identidades funcionais básicas que vamos considerar são

$$
\sum_{i \in \mathcal{I}} E_{i}\left(\bar{x}_{m}^{i}\right) x_{i}+\sum_{j \in \mathcal{J}} x_{j} F_{j}\left(\bar{x}_{m}^{j}\right)=0, \quad \text { para todo } \bar{x}_{m} \in A^{m}
$$




$$
\sum_{i \in \mathcal{I}} E_{i}\left(\bar{x}_{m}^{i}\right) x_{i}+\sum_{j \in \mathcal{J}} x_{j} F_{j}\left(\bar{x}_{m}^{j}\right) \in Z(A), \quad \text { para todo } \bar{x}_{m} \in A^{m}
$$

No caso $\mathcal{I}=\emptyset$ ou $\mathcal{J}=\emptyset$, a soma sobre estes conjuntos será entendida como sendo 0. Assim, por exemplo, se $J=\emptyset,(6.3)$ se reduz a

$$
\sum_{i \in \mathcal{I}} E_{i}\left(\bar{x}_{m}^{i}\right) x_{i}=0, \quad \text { para todo } \bar{x}_{m} \in A^{m}
$$

Um questionamento que segue naturalmente é que formas as funções $E_{i}$ e $F_{j}$ podem assumir, de modo que essas identidades funcionais sejam sempre satisfeitas para anéis arbitrários. A fim de prover uma solução padrão, consideraremos primeiramente um caso particular de (6.3). Seja

$$
E_{1}\left(x_{2}, x_{3}\right) x_{1}+E_{2}\left(x_{1}, x_{3}\right) x_{2}+x_{2} F_{2}\left(x_{1}, x_{3}\right)+x_{3} F_{3}\left(x_{1}, x_{2}\right)=0
$$

para todo $\bar{x}_{3} \in A^{3}$. Notemos que neste caso $\mathcal{I}=\{1,2\}$ e $\mathcal{J}=\{2,3\}$ e $m=3$. Com certeza, podemos incluir o termo $x_{2} p_{12}\left(x_{3}\right)$, onde $p_{12}: A \rightarrow A$ é uma função arbitrária, como um somando de $E_{1}\left(x_{2}, x_{3}\right)$, pois ele pode ser cancelado com um somando $-p_{12}\left(x_{3}\right) x_{1}$ em $F_{2}\left(x_{1}, x_{3}\right)$. Similarmente, o termo $x_{3} p_{13}\left(x_{2}\right)$ pode ser incluído em $E_{1}\left(x_{2}, x_{3}\right)$, uma vez que pode ser cancelado com $-p_{13}\left(x_{2}\right) x_{1}$ em $F_{3}\left(x_{1}, x_{2}\right)$. Além disso, o termo $x_{3} p_{23}\left(x_{1}\right)$ também pode aparecer em $E_{2}\left(x_{1}, x_{3}\right)$, e ser cancelado com $-p_{23}\left(x_{1}\right) x_{2}$ em $F_{3}\left(x_{1}, x_{2}\right)$. Observemos também que em $E_{2}\left(x_{1}, x_{3}\right)$ pode ser incluso um somando central, isto é, que pertence ao centro de $A$, da forma $\lambda_{2}\left(x_{1}, x_{3}\right)$, onde $\lambda_{2}: A^{2} \rightarrow Z(A)$ é uma aplicação, pois este pode ser cancelado com $-\lambda_{2}\left(x_{1}, x_{3}\right)$ em $F_{2}\left(x_{1}, x_{3}\right)$. Contudo, neste exemplo, não deve aparecer nenhum somando central $\lambda_{1}\left(x_{2}, x_{3}\right)$ em $E_{1}\left(x_{2}, x_{3}\right)$, pois $\lambda_{1}\left(x_{2}, x_{3}\right) x_{1}$ não pode ser cancelado. Em suma, construímos aplicações

$$
\begin{aligned}
& E_{1}\left(x_{2}, x_{3}\right)=x_{2} p_{12}\left(x_{3}\right)+x_{3} p_{13}\left(x_{2}\right), \\
& E_{2}\left(x_{1}, x_{3}\right)=x_{3} p_{23}\left(x_{1}\right)+\lambda_{2}\left(x_{1}, x_{3}\right), \\
& F_{2}\left(x_{1}, x_{3}\right)=-p_{12}\left(x_{3}\right) x_{1}-\lambda_{2}\left(x_{1}, x_{3}\right), \\
& F_{3}\left(x_{1}, x_{2}\right)=-p_{13}\left(x_{2}\right) x_{1}-p_{23}\left(x_{1}\right) x_{2},
\end{aligned}
$$

onde $p_{12}, p_{13}, p_{23}: A \rightarrow A$ e $\lambda_{2}: A^{2} \rightarrow Z(A)$ são aplicações arbitrárias, que formam sempre uma solução da I.F. anterior. Notemos que o somando central $\lambda_{i}$ aparece apenas quando $i \in \mathcal{I} \cap \mathcal{J}=\{2\}$. Chamamos esta solução de solução padrão para a identidade funcional anterior.

Voltando à identidade funcional em (6.3), podemos generalizar o processo anterior obtendo a solução padrão

$$
\begin{gathered}
E_{i}\left(\bar{x}_{m}^{i}\right)=\sum_{\substack{j \in \mathcal{J} \\
j \neq i}} x_{j} p_{i j}\left(\bar{x}_{m}^{i j}\right)+\lambda_{i}\left(\bar{x}_{m}^{i}\right), \quad i \in \mathcal{I}, \\
F_{j}\left(\bar{x}_{m}^{j}\right)=-\sum_{\substack{i \in \mathcal{I} \\
i \neq j}} p_{i j}\left(\bar{x}_{m}^{i j}\right) x_{i}-\lambda_{j}\left(\bar{x}_{m}^{j}\right), \quad j \in \mathcal{J}, \\
\lambda_{k}=0 \text { se } k \notin \mathcal{I} \cap \mathcal{J} .
\end{gathered}
$$

Agora, vamos introduzir uma noção essencial na teoria de identidades funcionais. Primeiramente, 
considere um anel $A$ e suponha que exista $d \in \mathbb{N}$ tal que

(a) sempre que $\max \{|\mathcal{I}|,|\mathcal{J}|\} \leq d,(6.3)$ admite somente a solução padrão (6.5).

Por exemplo, no caso $d=2$, se (a) é válida, então, $A$ não pode ser comutativo, pois $x y-y x=0$ proveria uma solução não padrão para a identidade funcional $E_{1}\left(x_{2}\right) x_{1}+E_{2}\left(x_{1}\right) x_{2}=0$, para todos $x_{1}, x_{2} \in A$. Observe também que se $A$ é um anel com elemento identidade, então, claramente (a) é verdadeira para $d=1$. De fato, neste caso as possíveis identidades funcionais seriam $E_{1}\left(\bar{x}_{m}^{1}\right) x_{1}=0$ $(\mathcal{I}=\{1\}$ e $\mathcal{J}=\emptyset), x_{1} F_{1}\left(\bar{x}_{m}^{1}\right)=0(\mathcal{I}=\emptyset$ e $\mathcal{J}=\{1\})$ e $E_{1}\left(\bar{x}_{m}^{1}\right) x_{1}+x_{1} F_{1}\left(\bar{x}_{m}^{1}\right)=0(\mathcal{I}=\mathcal{J}=\{1\})$. Na primeira I.F., escolhendo $x_{1}=1$, obtemos $E_{1}=0$, a qual é precisamente a solução padrão (6.5), pois $\mathcal{J}=\emptyset$. Igualmente, na segunda I.F., escolhendo $x_{1}=1$, temos a solução padrão $F_{j}=0$. Finalmente, na terceira I.F., também com $x_{1}=1$, obtemos $F_{1}=-E_{1} \in Z(A)$.

Além disso, suponha que também exista $d \in \mathbb{N}$ satisfazendo a condição

(b) sempre que $\max \{|\mathcal{I}|,|\mathcal{J}|\} \leq d-1,(6.4)$ admite somente a solução padrão (6.5).

Para $d \in \mathbb{N}$, dizemos que um anel $A$ é $d$-livre, ou mais precisamente que " $A$ é um subconjunto $d$-livre de si mesmo", se ambas condições (a) e (b) são válidas.

Exemplo 6.1.3. Se $C$ é um anel simples, comutativo e com elemento identidade, e $A=M_{n}(C)$, $n>1$, então, $A$ é simples. Portanto, $A$ é primo, e não é comutativo. Desse modo, pelos cálculos dos Exemplos 6.1.1 e 6.1.2, concluímos que as I.F.'s

$$
\begin{gathered}
E(x) y+F(y) x=0 \mathrm{e} \\
E(x) y+F(y) x \in Z(A),
\end{gathered}
$$

possuem apenas a solução padrão. Estes são dois dos cálculos necessários para se mostrar que o anel $A$ é 2-livre.

Vamos generalizar um pouco mais a noção de anéis $d$-livres. Seja $Q$ um anel com identidade e centro $C$. Fixamos $m$ um inteiro positivo, $S_{1}, S_{2}, \ldots, S_{m}$ conjuntos não vazios arbitrários, e $\alpha_{i}: S_{i} \rightarrow$ $Q, i=1,2, \ldots, m$, aplicações. Definimos

$$
R_{i}=\alpha_{i}\left(S_{i}\right), i=1, \ldots, m
$$

Escreveremos $\hat{S}$ para $S_{1} \times S_{2} \times \ldots \times S_{m}$ e $\hat{R}$ para $R_{1} \times R_{2} \times \ldots \times R_{m}$. Análoga à notação anterior, para $1 \leq i \leq m$, temos

$$
\hat{S}^{i}=\prod_{\substack{k=1 \\ k \neq i}}^{m} S_{k},
$$

e para $1 \leq i<j \leq m$, fixamos

$$
\hat{S}^{i j}=\hat{S}^{j i}=\prod_{\substack{k=1 \\ k \neq i, j}}^{m} S_{k} .
$$


Similarmente, definimos $\hat{R}^{i}$ e $\hat{R}^{i j}$. Para elementos $x_{i} \in S_{i}, i=1, \ldots, m$, vamos denotar

$$
\begin{aligned}
\bar{x}_{m} & =\left(x_{1}, \ldots, x_{m}\right) \in \hat{S} \\
\bar{x}_{m}^{i} & =\left(x_{1}, \ldots, x_{i-1}, x_{i+1}, \ldots, x_{m}\right) \in \hat{S}^{i} \\
\bar{x}_{m}^{i j}=\bar{x}_{m}^{j i} & =\left(x_{1}, \ldots, x_{i-1}, x_{i+1}, \ldots, x_{j-1}, x_{j+1}, \ldots, x_{m}\right) \in \hat{S}^{i j} .
\end{aligned}
$$

No caso $S=S_{1}=\ldots=S_{m}$, escrevemos $S^{m}$ ao invés de $\hat{S}$. A seguir, introduziremos dois tipos de funções fundamentais na teoria geral de identidades funcionais.

Dada uma sequência $\left(i_{1}, \ldots, i_{p}\right)$ de elementos distintos do conjunto $\{1,2, \ldots, m\}$, definimos uma função monomial $M: \hat{S} \rightarrow Q$ dada por $M\left(x_{1}, \ldots, x_{m}\right)=\alpha_{i_{1}}\left(x_{i_{1}}\right) \ldots \alpha_{i_{p}}\left(x_{i_{p}}\right)$. Dizemos que o "domínio" de $M, \operatorname{dom}(M)$, é o conjunto $\left\{i_{1}, i_{2}, \ldots, i_{p}\right\}, M$ é dito ter grau $p$ e denotamos grau $(M)=$ p. Em particular, para cada $1 \leq i \leq m$, temos $X_{i}: \hat{S} \rightarrow Q$ dada por $X_{i}\left(x_{1}, \ldots, x_{m}\right)=\alpha_{i}\left(x_{i}\right)$. Claramente a função $M$ definida acima é o produto $X_{i_{1}} X_{i_{2}} \ldots X_{i_{p}}$ das funções $X_{i_{k}}, k=1, \ldots, p$. A função $M: \bar{x}_{m} \mapsto 1$, será considerada como uma função monomial com $\operatorname{dom}(M)=\emptyset$ e $\operatorname{grau}(M)=0$. Para cada função monomial $M=X_{i_{1}} X_{i_{2}} \ldots X_{i_{p}}$, definimos

$$
\hat{S}^{M}=S_{j_{1}} \times S_{j_{2}} \times \ldots \times S_{j_{m-p}},
$$

onde $\left\{i_{1}, \ldots, i_{p}\right\} \cup\left\{j_{1}, \ldots, j_{m-p}\right\}$ é uma partição de $\{1,2, \ldots, m\}, j_{1}<j_{2}<\ldots<j_{m}$.

Dada uma função $B: S_{j_{1}} \times S_{j_{2}} \times \ldots \times S_{j_{n}} \rightarrow Q, 0 \leq n<m, j_{1}<j_{2}<\ldots<j_{n}$, substituímos $B$ pela função dada por

$$
\left(x_{1}, x_{2}, \ldots, x_{m}\right) \mapsto B\left(x_{j_{1}}, x_{j_{2}}, \ldots, x_{j_{n}}\right),
$$

a qual cometendo um pequeno abuso de notação continuaremos a denotar por $B$. No caso em que $n=0, B$ deve ser entendida como um elemento de $Q$.

Vamos introduzir um dos tipos básicos de funções que iremos considerar. Inicialmente, fixemos $n$ tal que $0 \leq n<m$. Em seguida, seja $\left\{i_{1}, \ldots, i_{p}\right\} \cup\left\{k_{1}, \ldots, k_{r}\right\} \cup\left\{j_{1}, \ldots, j_{n}\right\}$ uma partição de $\{1,2, \ldots, m\}$, com $p+r=m-n$ e $j_{1}<j_{2}<\ldots<j_{n}$. Observe que enquanto $n$ está fixo, $p$ e $r$ podem variar. Sejam $M$ e $N$ as funções monomiais $X_{i_{1}} X_{i_{2}} \ldots X_{i_{p}}$ e $X_{k_{1}} X_{k_{2}} \ldots X_{k_{r}}$, respectivamente. Note que $M N$ é novamente uma função monomial e $\hat{S}^{M N}=S_{j_{1}} \times S_{j_{2}} \times \ldots \times S_{j_{n}}$. Para cada par $M, N$, considere uma aplicação $B_{M, N}: \hat{S}^{M N} \rightarrow Q$, cuja extensão a $\hat{S}$

$$
\left(x_{1}, x_{2}, \ldots, x_{m}\right) \mapsto B_{M, N}\left(x_{j_{1}}, \ldots, x_{j_{n}}\right),
$$

continuaremos denotando por $B_{M, N}$, como indicado anteriormente. Desse modo, o tipo básico de funções que iremos considerar é

$$
\sum_{M, N} M B_{M, N} N
$$

Nesse somatório $M$ e $N$ são funções monomiais tais que $\operatorname{dom}(M) \cap \operatorname{dom}(N)=\emptyset$ e $\operatorname{grau}(M)+$ $\operatorname{grau}(N)=m-n$. Chamaremos essas funções de funções de núcleo. Para cada somando em (6.6) $B_{M, N}$ é chamada de função de meio, $M$ e $N$ são as funções monomiais à esquerda e à direita, respectivamente. As funções de meio possuem sempre aridade $n$ (fixado). Diremos que $B_{U, V}$ é uma função de meio mais à esquerda se $B_{M, N}=0$ sempre que $\operatorname{grau}(M)<\operatorname{grau}(U)$, isto é, $U$ tem grau mínimo dentre os monômios à esquerda que de fato "aparecem" em (6.6). De modo análogo, 


\section{define-se função de meio mais à direita.}

As funções básicas envolvidas na definição de conjuntos $d$-livres são exemplos de funções de núcleo. Por exemplo, considere o caso $S_{k}=R_{k}, \alpha_{k}=\mathrm{id}_{k}, k=1,2, \ldots, m$, e também a seguinte identidade

$$
\sum_{i \in \mathcal{I}} E_{i}\left(\bar{x}_{m}^{i}\right) x_{i}+\sum_{j \in \mathcal{J}} x_{j} F_{j}\left(\bar{x}_{m}^{j}\right)=0
$$

Os conjuntos $\mathcal{I}$ e $\mathcal{J}$ são subconjuntos de $\{1,2, \ldots, m\}, n=m-1$, com $p=0$ e $r=1$ no primeiro somatório, e $p=1$ e $r=0$ no segundo. Claramente, as $E_{i}$ 's são funções de meio mais à esquerda e as $F_{j}$ 's são funções de meio mais à direita. Podemos ainda reescrever esta identidade usando a "notação de função", o que usualmente faremos a partir de agora, da seguinte maneira

$$
\sum_{i \in \mathcal{I}} E_{1, X_{i}} X_{i}+\sum_{j \in \mathcal{J}} X_{j} F_{X_{j}, 1}=0
$$

Exemplo 6.1.4. A fim de ilustrar a notação acima, tome $m=2$. No caso $\mathcal{I}, \mathcal{J}=\{1,2\}$, temos a identidade funcional

$$
E_{1}\left(x_{2}\right) x_{1}+E_{2}\left(x_{1}\right) x_{2}+x_{1} F_{1}\left(x_{2}\right)+x_{2} F_{2}\left(x_{1}\right)=0
$$

que pode ser vista como uma função de núcleo igualada a 0 . As aplicações $E_{1}, E_{2}$ são funções de meio mais à esquerda, pois os monômios que multiplicam à esquerda tem grau 0 ; e as aplicações $F_{1}, F_{2}$ são funções de meio mais à direita. Todas elas tem aridade 1. Em notação de função, escrevemos

$$
E_{1, X_{1}} X_{1}+E_{1, X_{2}} X_{2}+X_{1} F_{X_{1}, 1}+X_{2} F_{X_{2}, 1}=0
$$

Nesse momento, outro tipo de funções que introduziremos são as chamadas quasi-polinomiais, definidas como segue. Seja $L=X_{i_{1}} \ldots X_{i_{p}}$ um monômio arbitrário de grau $p, 0 \leq p \leq m$, e considere uma aplicação

$$
\lambda_{L}: S_{j_{1}} \times S_{j_{2}} \times \ldots \times S_{j_{n}} \rightarrow C,
$$

onde $C$ é o centro do anel $Q$, e $\left\{i_{1}, \ldots, i_{p}\right\} \cup\left\{j_{1}, \ldots, j_{n}\right\}$ é uma partição de $\{1,2, \ldots, m\}$ e $j_{1}<$ $j_{2}<\ldots<j_{n}$ (se $p=m$, então, $\lambda_{L}$ é um elemento de $C$ ). Cometendo o mesmo abuso de notação anterior, continuaremos denotando por $\lambda_{L}$ a função dada por $\bar{x}_{m} \mapsto \lambda_{L}\left(x_{j_{1}}, \ldots, x_{j_{n}}\right)$, que tem como domínio $\hat{S}$. Pela notação de função, dizemos que uma função do tipo

$$
P=\sum_{L} \lambda_{L} L
$$

é chamada de quasi-polinomial. Os $\lambda_{L}$ 's são os coeficientes de $P$ e $\lambda_{1}$, o coeficiente associado ao monômio $L=1$, é o coeficiente central. Se pelo menos um dos $\lambda_{L}$ 's é não nulo, dizemos que o grau de $P$ é $m$. No caso em que todos os $\lambda_{L}$ 's sejam nulos, definimos o grau de $P$ como $-\infty$. Uma quasi-polinomial de grau 0 é simplesmente um elemento não nulo de $C$, o qual identificamos com uma função constante. Por exemplo, uma quasi-polinomial de grau 1 seria uma função do tipo

$$
P=\lambda_{X_{1}} X_{1}+\lambda_{1},
$$


ou seja,

$$
P\left(x_{1}\right)=\lambda \alpha_{1}\left(x_{1}\right)+\mu\left(x_{1}\right)
$$

onde $\lambda_{X_{1}}=\lambda, \lambda_{1}=\mu, \lambda \in C$ e $\mu: S_{1} \rightarrow C$ é o coeficiente central. Além disso, $\lambda$ e $\mu$ não são simultaneamente nulos.

Uma quasi-polinomial de grau 2 pode ser descrita como

$$
P=\lambda_{X_{1} X_{2}} X_{1} X_{2}+\lambda_{X_{2} X_{1}} X_{2} X_{1}+\lambda_{X_{2}} X_{2}+\lambda_{X_{1}} X_{1}+\lambda_{1}
$$

isto é,

$$
P\left(x_{1}, x_{2}\right)=\lambda \alpha_{1}\left(x_{1}\right) \alpha_{2}\left(x_{2}\right)+\lambda^{\prime} \alpha_{2}\left(x_{2}\right) \alpha_{1}\left(x_{1}\right)+\mu\left(x_{1}\right) \alpha_{2}\left(x_{2}\right)+\mu^{\prime}\left(x_{2}\right) \alpha_{1}\left(x_{1}\right)+\gamma\left(x_{1}, x_{2}\right),
$$

onde $\lambda_{X_{1} X_{2}}=\lambda, \lambda_{X_{2} X_{1}}=\lambda^{\prime}, \lambda_{X_{2}}=\mu, \lambda_{X_{1}}=\mu^{\prime}, \lambda_{1}=\gamma, \lambda_{i} \in C, \mu_{i}: S_{i} \rightarrow C, i=1,2$, e $\gamma: S_{1} \times S_{2} \rightarrow C$.

O tipo mais geral de I.F. que iremos considerar no que se segue é uma em que se iguala uma função de núcleo a uma quasi-polinomial:

$$
\sum_{M, N} M B_{M, N} N=\sum_{L} \lambda_{L} L
$$

Nesta I.F., entenderemos que a quasi-polinomial é conhecida, enquanto as funções de meio da função de núcleo são desconhecidas. Mas, antes disso, vamos estabelecer a noção de par $d$-livre, para $d \in \mathbb{N}$.

Vamos adotar as mesmas notações fixadas anteriormente: $S_{1}, S_{2}, \ldots, S_{m}$ são conjuntos não vazios quaisquer, $\alpha_{k}: S_{k} \rightarrow Q, 1 \leq k \leq m$, são funções arbitrárias e $R_{k}=\alpha_{k}\left(S_{k}\right) \subseteq Q$. Além disso, fixamos

$$
\hat{\alpha}=\left(\alpha_{1}, \ldots, \alpha_{m}\right)
$$

e consideramos $\hat{\alpha}$ como uma aplicação sobrejetora de $\hat{S}$ em $\hat{R}$, dada por

$$
\hat{\alpha}\left(x_{1}, x_{2}, \ldots, x_{m}\right)=\left(\alpha_{1}\left(x_{1}\right), \alpha_{2}\left(x_{2}\right), \ldots, \alpha_{m}\left(x_{m}\right)\right) .
$$

As definições de $\hat{\alpha}^{i}: \hat{S}^{i} \rightarrow \hat{R}^{i}$ e de $\hat{\alpha}^{i j}: \hat{S}^{i j} \rightarrow \hat{R}^{i j}$, para $i, j \in\{1,2, \ldots, m\}$, seguem a mesma lógica das definições dadas acima para $\hat{S}^{i}$ e para $\hat{S}^{i j}$.

Sejam $\mathcal{I}, \mathcal{J} \subseteq\{1,2, \ldots, m\}, E_{i}: \hat{S}^{i} \rightarrow Q, i \in \mathcal{I}$, e $F_{j}: \hat{S}^{j} \rightarrow Q, j \in \mathcal{J}$. Considere as identidades funcionais

$$
\begin{aligned}
\sum_{i \in \mathcal{I}} E_{i}\left(\bar{x}_{m}^{i}\right) \alpha_{i}\left(x_{i}\right)+\sum_{j \in \mathcal{J}} \alpha_{j}\left(x_{j}\right) F_{j}\left(\bar{x}_{m}^{j}\right) & =0, \quad \text { para todo } \bar{x}_{m} \in \hat{S} \\
\sum_{i \in \mathcal{I}} E_{i}\left(\bar{x}_{m}^{i}\right) \alpha_{i}\left(x_{i}\right)+\sum_{j \in \mathcal{J}} \alpha_{j}\left(x_{j}\right) F_{j}\left(\bar{x}_{m}^{j}\right) & \in C, \quad \text { para todo } \bar{x}_{m} \in \hat{S}
\end{aligned}
$$


Prosseguimos naturalmente definindo uma solução padrão de (6.8) e (6.9) por

$$
\begin{gathered}
E_{i}\left(\bar{x}_{m}^{i}\right)=\sum_{\substack{j \in \mathcal{J} \\
j \neq i}} \alpha_{j}\left(x_{j}\right) p_{i j}\left(\bar{x}_{m}^{i j}\right)+\lambda_{i}\left(\bar{x}_{m}^{i}\right), \quad i \in \mathcal{I}, \\
F_{j}\left(\bar{x}_{m}^{j}\right)=-\sum_{\substack{i \in \mathcal{I} \\
i \neq j}} p_{i j}\left(\bar{x}_{m}^{i j}\right) \alpha_{i}\left(x_{i}\right)-\lambda_{j}\left(\bar{x}_{m}^{j}\right), \quad j \in \mathcal{J}, \\
\lambda_{k}=0 \text { se } k \notin \mathcal{I} \cap \mathcal{J},
\end{gathered}
$$

onde $p_{i j}: \hat{S}^{i j} \rightarrow Q, i \in \mathcal{I}, j \in \mathcal{J}, i \neq j$, e $\lambda_{k}: \hat{S}^{k} \rightarrow C, k \in \mathcal{I} \cup \mathcal{J}$.

Definição 6.1.2. Um par $(\hat{S}, \hat{\alpha})$ é dito ser $d$-livre, onde $d \in \mathbb{N}$, se para todo $\mathcal{I}, \mathcal{J} \subseteq\{1,2, \ldots, m\}$, as seguintes condições são satisfeitas:

(a) se $\max \{|\mathcal{I}|,|\mathcal{J}|\} \leq d$, então, (6.8) implica (6.10).

(b) se $\max \{|\mathcal{I}|,|\mathcal{J}|\} \leq d-1$, então, (6.9) implica (6.10).

Um importante caso particular é quando $S_{k}=R_{k}$ e $\alpha_{k}=\operatorname{id}_{S_{k}}$, para todo $k \in\{1,2, \ldots, m\}$. Neste caso, se as condições (a) e (b) da definição anterior são satisfeitas para quaisquer $\mathcal{I}, \mathcal{J} \subseteq$ $\{1,2, \ldots, m\}$, dizemos que $\hat{R}$ é um subconjunto $d$-livre de $Q^{m}$. Se $R_{i}=R$, para todo $i=1, \ldots, m$, dizemos que $R^{m}$ é um subconjunto $d$-livre de $Q^{m}$ ou que $R$ é um subconjunto $d$-livre de $Q$. Para tais subconjuntos temos o seguinte lema.

Lema 6.1.1. Se $R$ é um subconjunto $d$-livre de $Q, d \geq 1$, onde $Q$ é um anel com elemento identidade e centro $C$, então, as seguintes afirmações são satisfeitas:

(i) Se $q \in Q$ é tal que $q R=0$ (ou $R q=0$ ), então, $q=0$;

(ii) Se $d \geq 2$ e $q \in Q$ é tal que $q R \subseteq C$ (ou $R q \subseteq C$ ), então, $q=0$;

(iii) Se $q \in Q$ é tal que $[q, R]=0$, então, $q \in C$;

(iv) Se $d \geq 2$ e $q \in Q$ é tal que $[q, R] \subseteq C$, então, $q \in C$;

(v) Se $\lambda \in C^{*}$, isto é, $\lambda$ é invertível em $C$, então, $\lambda R$ é um subconjunto $d$-livre de $Q$.

Demonstração. Para provar (i), observe que $q x=0$, para todo $x \in R$, é uma identidade funcional em $R(\mathcal{I}=\{1\}$ e $\mathcal{J}=\emptyset)$. Assim, como $R$ é $d$-livre $(d \geq 1)$, segue $q=0$. A prova de (ii) é análoga a de (i). Para (iii), temos a I.F. $q x-x q=0$, para todo $x \in R(\mathcal{I}=\mathcal{J}=\{1\})$. Desse modo, como $R$ é $d$-livre, temos $q \in C$. Analogamente, prova-se (iv). Finalmente, para verificar que vale (v), basta observarmos que a aplicação $x \mapsto \lambda x, x \in R$, dá origem a uma correspondência biunívoca entre identidades funcionais e soluções sobre $R$ e sobre $\lambda R$.

Antes de enunciarmos e provarmos o próximo teorema, que relaciona pares $d$-livres com subconjuntos $d$-livres, consideremos o seguinte exemplo.

Exemplo 6.1.5. Sejam $R$ um subgrupo aditivo de $Q$, onde $Q$ é um anel com identidade e centro $C$, e $F: R \rightarrow Q$ uma aplicação aditiva satisfazendo

$$
[F(x), x]=0, \quad \text { para todo } x \in R \text {. }
$$


Um exemplo canônico de uma tal aplicação é

$$
F(x)=\lambda x+\mu(x), \lambda \in C, \mu: R \rightarrow C,
$$

onde $\mu$ é também aditiva. Nosso objetivo é encontrar hipóteses sobre $R$, que nos permitam concluir que $F$ é da forma padrão apresentada em (6.12). Primeiramente, observemos que a identidade funcional em (6.11) não se encontra em uma forma em que seja possível usar as condições de $d$-liberdade. Porém, como $F$ é aditiva, podemos linearizá-la, o que resulta

$$
F\left(x_{2}\right) x_{1}+F\left(x_{1}\right) x_{2}-x_{1} F\left(x_{2}\right)-x_{2} F\left(x_{1}\right)=0, \quad \text { para todos } x_{1}, x_{2} \in R
$$

Assumindo que $R$ é um subconjunto 2-livre de $Q$, segue que existem $p_{12}, p_{21} \in Q$ e $\lambda_{1}, \lambda_{2}: R \rightarrow C$ tais que

$$
\begin{aligned}
& E_{1}\left(x_{2}\right)=F\left(x_{2}\right)=x_{2} p_{12}+\lambda_{1}\left(x_{2}\right), \\
& E_{2}\left(x_{1}\right)=F\left(x_{1}\right)=x_{1} p_{21}+\lambda_{2}\left(x_{1}\right), \\
& F_{1}\left(x_{2}\right)=-F\left(x_{2}\right)=-p_{21} x_{2}-\lambda_{1}\left(x_{2}\right), \\
& F_{2}\left(x_{1}\right)=-F\left(x_{1}\right)=-p_{12} x_{1}-\lambda_{2}\left(x_{1}\right),
\end{aligned}
$$

para todos $x_{1}, x_{2} \in R$. Escolhendo $x_{1}=x_{2}=x$ e comparando, por exemplo, a primeira com a última igualdade, obtemos $\left[x, p_{12}\right]=\lambda_{2}(x)-\lambda_{1}(x) \in C$. Assim, por (iii) do Lema 6.1.1, temos $p_{12} \in C$, e portanto, $\lambda_{2}=\lambda_{1}$. Analogamente, por (i) do Lema 6.1.1, chegamos a $p_{21}=p_{12}$. Agora, denotamos $\lambda=p_{12}=p_{21} \in C$ e $\mu=\lambda_{1}=\lambda_{2}$, e concluímos que, de fato, $F$ tem a forma dada em (6.12). Desse modo, se $R$ é 2-livre, (6.11) implica (6.12). Prosseguindo, vamos generalizar a identidade funcional em (6.11). Sejam $S$ um grupo aditivo (não necessariamente contido em $Q$ ), $\alpha: S \rightarrow Q$ e $F: S \rightarrow Q$ aplicações aditivas satisfazendo

$$
[F(x), \alpha(x)]=0, \quad \text { para todo } x \in S \text {. }
$$

Definimos uma solução padrão de (6.13) por

$$
F(x)=\lambda \alpha(x)+\mu(x), \quad \lambda \in C, \mu: S \rightarrow C .
$$

Observe que a igualdade em (6.14) equivale a dizer que $F$ é uma quasi-polinomial de grau 1. A questão que naturalmente se coloca é se $\alpha(S)$ é um subconjunto 2-livre de $Q$, então, (6.13) implica (6.14). Observe que se $\alpha$ for injetiva, então, a resposta é positiva, pois (6.13) se reduz a $\left[\left(F \alpha^{-1}\right)(y), y\right]=0$, para todo $y \in \alpha(S)$, e voltamos ao caso anterior, com $F \alpha^{-1}$ tomando o papel de $F$. Contudo, se $\alpha$ não é injetiva, o problema não parece ser inteiramente óbvio, pois não recaímos no caso anterior. De qualquer maneira, a resposta é positiva e segue facilmente do próximo teorema, o qual descreve um caso muito mais geral.

Teorema 6.1.1. (Brešar, Chebotar e Martindale, 3rd, [8]) O par ( $\hat{S}, \hat{\alpha})$ é $d$-livre se, e somente se, o conjunto $\hat{R}$ é $d$-livre, onde $d \in \mathbb{N}$.

Demonstração. Primeiramente note que existem aplicações $\beta_{k}: R_{k} \rightarrow S_{k}$ tais que $\alpha_{k} \beta_{k}=\mathrm{id}_{R_{k}}$, para $k=1,2, \ldots, m$, pois $\alpha_{k}$ é sobrejetora. Assim, $\hat{\beta}=\left(\beta_{1}, \beta_{2}, \ldots, \beta_{m}\right)$ é uma aplicação injetora de $\hat{R}$ em $\hat{S}$, tal que $\hat{\alpha} \hat{\beta}=\operatorname{id}_{\hat{R}}$. 
Primeiramente, suponha que $(\hat{S}, \hat{\alpha})$ é $d$-livre. Vamos mostrar que $\hat{R}$ satisfaz a condição (a) da Definição 6.1.2. Assim, iniciamos com a identidade funcional

$$
\sum_{i \in \mathcal{I}} E_{i}\left(\bar{y}_{m}^{i}\right) y_{i}+\sum_{j \in \mathcal{J}} y_{j} F_{j}\left(\bar{y}_{m}^{j}\right)=0
$$

para todo $\bar{y}_{m} \in \hat{R}$, onde $\mathcal{I}$ e $\mathcal{J}$ são subconjuntos de $\{1,2, \ldots, m\}$, tais que $\max \{|\mathcal{I}|,|\mathcal{J}|\} \leq d$, $E_{i}: \hat{R}^{i} \rightarrow Q, F_{j}: \hat{R}^{j} \rightarrow Q$ são aplicações. Defina $\tilde{E}_{i}: \hat{S}^{i} \rightarrow Q$ como sendo a composição $E_{i} \hat{\alpha}^{i}$ e $\tilde{F}_{j}: \hat{S}^{j} \rightarrow Q$ como sendo a composição $F_{j} \hat{\alpha}^{j}$. Portanto, temos $\tilde{E}_{i} \hat{\beta}^{i}=E_{i} \hat{\alpha}^{i} \hat{\beta}^{i}=E_{i}$ e $\tilde{F}_{j} \hat{\beta}^{j}=$ $F_{j} \hat{\alpha}^{j} \hat{\beta}^{j}=F_{j}$. Desse modo, segue

$$
\sum_{i \in \mathcal{I}} \tilde{E}_{i}\left(\bar{x}_{m}^{i}\right) \alpha_{i}\left(x_{i}\right)+\sum_{j \in \mathcal{J}} \alpha_{j}\left(x_{j}\right) \tilde{F}_{j}\left(\bar{x}_{m}^{j}\right)=0, \quad \text { para todo } \bar{x}_{m} \in \hat{S}
$$

Assim, como $(\hat{S}, \hat{\alpha})$ é $d$-livre, existem $\tilde{p}_{i j}: \hat{S}^{i j} \rightarrow Q, i \in \mathcal{I}, j \in \mathcal{J}, i \neq j$, e $\tilde{\lambda}_{k}: \hat{S}^{k} \rightarrow C$, $k \in \mathcal{I} \cup \mathcal{J}$, tais que

$$
\begin{gathered}
\tilde{E}_{i}\left(\bar{x}_{m}^{i}\right)=\sum_{\substack{j \in \mathcal{J} \\
j \neq i}} \alpha_{j}\left(x_{j}\right) \tilde{p}_{i j}\left(\bar{x}_{m}^{i j}\right)+\tilde{\lambda}_{i}\left(\bar{x}_{m}^{i}\right), i \in \mathcal{I}, \\
\left.\tilde{F}_{j}^{(} \bar{x}_{m}^{j}\right)=-\sum_{\substack{i \in \mathcal{I} \\
i \neq j}} \tilde{p}_{i j}\left(\bar{x}_{m}^{i j}\right) \alpha_{i}\left(x_{i}\right)-\tilde{\lambda}_{j}\left(\bar{x}_{m}^{j}\right), j \in \mathcal{J}, \\
\tilde{\lambda}_{k}=0, \text { se } k \notin \mathcal{I} \cap \mathcal{J} .
\end{gathered}
$$

Agora, defina $p_{i j}=\tilde{p}_{i j} \hat{\beta}^{i j}$ e $\lambda_{k}=\tilde{\lambda}_{k} \hat{\beta}^{k}$. Em vista de que vale $\hat{\alpha}^{k} \hat{\beta}^{k}=\mathrm{id}_{\hat{R}_{k}}$, temos

$$
\begin{aligned}
E_{i}\left(\bar{y}_{m}^{i}\right) & =\tilde{E}_{i} \hat{\beta}^{i}\left(\bar{y}_{m}^{i}\right)=\tilde{E}_{i}\left(\hat{\beta}^{i}\left(\bar{y}_{m}^{i}\right)\right) \\
& =\sum_{\substack{j \in \mathcal{J} \\
j \neq i}} \alpha_{j}\left(\beta_{j}\left(y_{j}\right)\right) \tilde{p}_{i j}\left(\hat{\beta}^{i j}\left(\bar{y}_{m}^{i j}\right)\right)+\tilde{\lambda}_{i}\left(\hat{\beta}^{i}\left(\bar{y}_{m}^{i}\right)\right) \\
& =\sum_{\substack{j \in \mathcal{J} \\
j \neq i}} y_{j} p_{i j}\left(\bar{y}_{m}^{i j}\right)+\lambda_{i}\left(\bar{y}_{m}^{i}\right), \quad \mathrm{e} \\
F_{j}\left(\bar{y}_{m}^{i}\right) & =\tilde{F}_{j} \hat{\beta}^{j}\left(\bar{y}_{m}^{j}\right)=\tilde{F}_{j}\left(\hat{\beta}^{j}\left(\bar{y}_{m}^{j}\right)\right) \\
& =-\sum_{\substack{i \in \mathcal{I} \\
i \neq j}} \tilde{p}_{i j}\left(\hat{\beta}^{i j}\left(\bar{y}_{m}^{i j}\right)\right) \alpha_{i}\left(\beta_{i}\left(y_{i}\right)\right)-\tilde{\lambda}_{j}\left(\hat{\beta}^{j}\left(\bar{y}_{m}^{j}\right)\right) \\
& =-\sum_{\substack{i \in \mathcal{I} \\
i \neq j}} p_{i j}\left(\bar{y}_{m}^{i j}\right) y_{i}-\lambda_{j}\left(\bar{y}_{m}^{j}\right),
\end{aligned}
$$

com $p_{i j}: \hat{R}^{i j} \rightarrow Q, i \in \mathcal{I}, j \in \mathcal{J}, i \neq j$, e $\lambda_{k}: \hat{R}^{k} \rightarrow C, k \in \mathcal{I} \cup \mathcal{J}, \lambda_{k}=0$, se $k \notin \mathcal{I} \cap \mathcal{J}$. Assim, a I.F. em (6.15) possui apenas a solução padrão. A condição (b) pode ser verificada de maneira análoga.

Prosseguindo, assuma que $\hat{R}$ é $d$-livre. Assim, como anteriormente, verificaremos a condição (a) da Definição 6.1 .2 e (b) pode ser provada de maneira similar. Queremos mostrar que a seguinte IF em $\hat{S}$ :

$$
D\left(\bar{x}_{m}\right)=\sum_{i \in \mathcal{I}} E_{i}\left(\bar{x}_{m}^{i}\right) \alpha_{i}\left(x_{i}\right)+\sum_{j \in \mathcal{J}} \alpha_{j}\left(x_{j}\right) F_{j}\left(\bar{x}_{m}^{j}\right)=0
$$


onde $E_{i}: \hat{S}^{i} \rightarrow Q$ e $F_{j}: \hat{S}^{j} \rightarrow Q$ são aplicações, possui somente a solução padrão. A prova disso será feita por indução em $|\mathcal{I} \cup \mathcal{J}|$. Se $|\mathcal{I} \cup \mathcal{J}|=1$, então, como $\hat{R}$ é 1 -livre, pois $d \geq 1$ e se $d^{\prime} \leq d$ claramente temos que $\hat{R} d$-livre implica $\hat{R} d^{\prime}$-livre, o que nos permite concluir $E_{1}\left(S_{2} \times \ldots \times S_{m}\right) \subseteq C$, com $F_{1}=-E_{1}$. De fato, se $\mathcal{I}=\{1\}$ e $\mathcal{J}=\emptyset$, vale $E_{1}\left(\bar{x}_{m}^{1}\right) x_{1}=0$ e temos a solução padrão $E_{1}\left(\bar{x}_{m}^{1}\right)=$ $0 \in C$. O caso $\mathcal{I}=\emptyset$ e $\mathcal{J}=\{1\}$ é análogo. Se $\mathcal{I}=\mathcal{J}=\{1\}$, temos $E_{1}\left(\bar{x}_{m}^{1}\right) x_{1}+x_{1} F_{1}\left(\bar{x}_{m}^{1}\right)=0$, e segue a solução padrão $E_{1}\left(\bar{x}_{m}^{1}\right)=\lambda_{1}\left(\bar{x}_{m}^{1}\right) \in C$ e $F_{1}=-\lambda_{1}=-E_{1}$. Agora, vamos primeiro lidar com o caso $\mathcal{I} \cup \mathcal{J}=\{1,2, \ldots, m\}$. Fixe $\epsilon_{k}=\beta_{k} \alpha_{k}$ e observe $\alpha_{k} \epsilon_{k}=\alpha_{k}$. Afirmamos que $E_{i}$ tem a forma padrão quando restrita ao conjunto $\epsilon_{1}\left(S_{1}\right) \times \ldots \times \epsilon_{i-1}\left(S_{i-1}\right) \times \epsilon_{i+1}\left(S_{i+1}\right) \times \ldots \times \epsilon_{m}\left(S_{m}\right)$, e o análogo é válido para $F_{j}$. Com efeito, definindo $\bar{E}_{i}=E_{i} \hat{\beta}^{i}, \bar{F}_{j}=F_{j} \hat{\beta}^{j}$, em vista de que para todo $y_{i} \in R_{i}$, temos $y_{i}=\alpha_{i}\left(x_{i}\right)$, onde $x_{i}=\beta_{i}\left(y_{i}\right)$, segue que é valida a seguinte I.F.:

$$
\begin{aligned}
\sum_{i \in \mathcal{I}} \bar{E}_{i}\left(\bar{y}_{m}^{i}\right) y_{i}+\sum_{j \in \mathcal{J}} y_{j} \bar{F}_{j}\left(\bar{y}_{m}^{j}\right) & =\sum_{i \in \mathcal{I}} E_{i} \hat{\beta}^{i}\left(\bar{y}_{m}^{i}\right) y_{i}+\sum_{j \in \mathcal{J}} y_{j} F_{j} \hat{\beta}^{j}\left(\bar{y}_{m}^{j}\right) \\
& =\sum_{i \in \mathcal{I}} E_{i}\left(\bar{x}_{m}^{i}\right) \alpha_{i}\left(x_{i}\right)+\sum_{j \in \mathcal{J}} \alpha_{j}\left(x_{j}\right) F_{j}\left(\bar{x}_{m}^{j}\right)=0
\end{aligned}
$$

para qualquer $\bar{y}_{m} \in \hat{R}$. Assim, como $\hat{R}$ é $d$-livre, existem $\bar{p}_{i j}: \hat{R}^{i j} \rightarrow Q, i \in \mathcal{I}, j \in \mathcal{J}, i \neq j$, e $\bar{\lambda}_{k}: \hat{R}^{k} \rightarrow C, k \in \mathcal{I} \cup \mathcal{J}$, tais que

$$
\begin{gathered}
\bar{E}_{i}\left(\bar{y}_{m}^{i}\right)=\sum_{\substack{j \in \mathcal{J} \\
j \neq i}} y_{j} \bar{p}_{i j}\left(\bar{y}_{m}^{i j}\right)+\bar{\lambda}_{i}\left(\bar{y}_{m}^{i}\right), \quad i \in \mathcal{I}, \\
\bar{F}_{j}\left(\bar{y}_{m}^{j}\right)=-\sum_{\substack{i \in \mathcal{I} \\
i \neq j}} \bar{p}_{i j}\left(\bar{y}_{m}^{i j}\right) y_{i}-\bar{\lambda}_{j}\left(\bar{y}_{m}^{j}\right), \quad j \in \mathcal{J}, \\
\bar{\lambda}_{k}=0, \quad \text { se } k \notin \mathcal{I} \cap \mathcal{J} .
\end{gathered}
$$

Agora, defina $p_{i j}=\bar{p}_{i j} \hat{\alpha}^{i j}$ e $\lambda_{k}=\bar{\lambda}_{k} \hat{\alpha}^{k}$. Para $x_{i} \in S_{i}$, sejam $u_{i}=\epsilon_{i}\left(x_{i}\right)$ e $y_{i}=\alpha_{i}\left(u_{i}\right)$, o que implica $\alpha_{i}\left(u_{i}\right)=\alpha_{i} \epsilon_{i}\left(x_{i}\right)=\alpha_{i}\left(x_{i}\right)$. Consequentemente,

$$
\begin{aligned}
E_{i}\left(\bar{u}_{m}^{i}\right) & =E_{i} \hat{\epsilon}^{i}\left(\bar{x}_{m}^{i}\right)=E_{i} \hat{\beta}^{i}\left(\hat{\alpha}^{i}\left(\bar{x}_{m}^{i}\right)\right)=\bar{E}_{i}\left(\hat{\alpha}^{i}\left(\bar{x}_{m}^{i}\right)\right) \\
& =\sum_{\substack{j \in \mathcal{J} \\
j \neq i}} \alpha_{j}\left(x_{j}\right) \bar{p}_{i j}\left(\hat{\alpha}^{i j}\left(\bar{x}_{m}^{i j}\right)\right)+\bar{\lambda}_{i}\left(\hat{\alpha}^{i}\left(\bar{x}_{m}^{i}\right)\right) \\
& =\sum_{\substack{j \in \mathcal{J} \\
j \neq i}}^{j} \alpha_{j}\left(u_{j}\right) \bar{p}_{i j}\left(\hat{\alpha}^{i j}\left(\bar{u}_{m}^{i j}\right)\right)+\bar{\lambda}_{i}\left(\hat{\alpha}^{i}\left(\bar{u}_{m}^{i}\right)\right) \\
& =\sum_{\substack{j \in \mathcal{J} \\
j \neq i}} \alpha_{j}\left(u_{j}\right) p_{i j}\left(\bar{u}_{m}^{i j}\right)+\lambda_{i}\left(\bar{u}_{m}^{i}\right),
\end{aligned}
$$


para cada $i \in \mathcal{I}$ e

$$
\begin{aligned}
F_{j}\left(\bar{u}_{m}^{j}\right) & =F_{j} \hat{\epsilon}^{j}\left(\bar{x}_{m}^{j}\right)=F_{j} \hat{\beta}^{j}\left(\hat{\alpha}^{j}\left(\bar{x}_{m}^{j}\right)\right)=\bar{F}_{j}\left(\hat{\alpha}^{j}\left(\bar{x}_{m}^{j}\right)\right) \\
& =-\sum_{\substack{i \in \mathcal{I} \\
i \neq j}} \bar{p}_{i j}\left(\hat{\alpha}^{i j}\left(\bar{x}_{m}^{i j}\right)\right) \alpha_{j}\left(x_{j}\right)-\bar{\lambda}_{j}\left(\hat{\alpha}^{j}\left(\bar{x}_{m}^{j}\right)\right) \\
& =-\sum_{\substack{i \in \mathcal{I} \\
i \neq j}}^{i j} \bar{p}_{i j}\left(\hat{\alpha}^{i j}\left(\bar{u}_{m}^{i j}\right)\right) \alpha_{j}\left(u_{j}\right)-\bar{\lambda}_{j}\left(\hat{\alpha}^{j}\left(\bar{u}_{m}^{j}\right)\right) \\
& =-\sum_{\substack{i \in \mathcal{I} \\
i \neq j}} p_{i j}\left(\bar{u}_{m}^{i j}\right) \alpha_{i}\left(u_{i}\right)-\lambda_{j}\left(\bar{u}_{m}^{j}\right),
\end{aligned}
$$

para cada $j \in \mathcal{J}$. Além disso, $\lambda_{k}=0$, se $k \notin \mathcal{I} \cap \mathcal{J}$, e nossas afirmações estão provadas. A prova estaria completa se cada $\epsilon_{k}$ fosse $\operatorname{id}_{S_{k}}$, isto é, se $\alpha$ fosse injetora, mas não estamos assumindo esta hipótese.

Para cada $s=1,2, \ldots, m$, definimos $\mathcal{I}_{s}=\mathcal{I} \backslash\{s\}$ e $\mathcal{J}_{s}=\mathcal{J} \backslash\{s\}$. Além disso, dado $\bar{x}_{m} \in \hat{S}$, para cada $s=0,1,2, \ldots, m$, definimos

$$
\tilde{x}_{s}=\left(x_{1}, \ldots, x_{s}, \epsilon_{s+1}\left(x_{s+1}\right), \ldots, \epsilon_{m}\left(x_{m}\right)\right) .
$$

Note que vale $\tilde{x}_{s}^{s}=\left(x_{1}, \ldots, x_{s-1}, \epsilon_{s+1}\left(x_{s+1}\right), \ldots, \epsilon_{m}\left(x_{m}\right)\right)=\tilde{x}_{s-1}^{s}$, o que implica

$$
E_{s}\left(\tilde{x}_{s}^{s}\right)=E_{s}\left(\tilde{x}_{s-1}^{s}\right) \text { e } F_{s}\left(\tilde{x}_{s}^{s}\right)=F_{s}\left(\tilde{x}_{s-1}^{s}\right)
$$

Esta observação junto com a igualdade $\alpha_{k}\left(x_{k}\right)=\alpha_{k}\left(\epsilon_{k}\left(x_{k}\right)\right)$, para todo $k$, nos permite concluir

$$
\begin{aligned}
0 & =D\left(\tilde{x}_{s}\right)-D\left(\tilde{x}_{s-1}\right) \\
& =\sum_{i \in \mathcal{I}} E_{i}\left(\tilde{x}_{s}^{i}\right) \alpha_{i}\left(\tilde{x}_{i}\right)+\sum_{j \in \mathcal{J}} \alpha_{j}\left(\tilde{x}_{j}\right) F_{j}\left(\tilde{x}_{s}^{j}\right)-\sum_{i \in \mathcal{I}} E_{i}\left(\tilde{x}_{s-1}^{i}\right) \alpha_{i}\left(\tilde{x}_{i}\right)-\sum_{j \in \mathcal{J}} \alpha_{j}\left(\tilde{x}_{j}\right) F_{j}\left(\tilde{x}_{s-1}^{j}\right) \\
& =\sum_{i \in \mathcal{I}}\left(E_{i}\left(\tilde{x}_{s}^{i}\right)-E_{i}\left(\tilde{x}_{s-1}^{i}\right)\right) \alpha_{i}\left(\tilde{x}_{i}\right)+\sum_{j \in \mathcal{J}} \alpha_{j}\left(\tilde{x}_{j}\right)\left(F_{j}\left(\tilde{x}_{s}^{j}\right)-F_{j}\left(\tilde{x}_{s-1}^{j}\right)\right) \\
& =\sum_{i \in \mathcal{I}_{s}} G_{s i}\left(\bar{x}_{m}^{i}\right) \alpha_{i}\left(x_{i}\right)+\sum_{j \in \mathcal{J}_{s}} \alpha_{j}\left(x_{j}\right) H_{s j}\left(\bar{x}_{m}^{j}\right)
\end{aligned}
$$

onde $G_{s i}\left(\bar{x}_{m}^{i}\right)=E_{i}\left(\tilde{x}_{s}^{i}\right)-E_{i}\left(\tilde{x}_{s-1}^{i}\right), H_{s j}\left(\bar{x}_{m}^{j}\right)=F_{j}\left(\tilde{x}_{s}^{j}\right)-F_{j}\left(\tilde{x}_{s-1}^{j}\right), \tilde{x}_{i}=x_{i}$ se $i \leq s$ e $\tilde{x}_{i}=\epsilon_{i}\left(x_{i}\right)$ se $i \geq s$.

Além disso, pela hipótese de indução, cada $G_{s i}$ e $H_{s j}$ pode ser escrito na forma padrão. Para $\bar{x}_{m} \in \hat{S}$, temos

$$
\begin{aligned}
& E_{i}\left(\bar{x}_{m}^{i}\right)=\sum_{s=1}^{m} G_{s i}\left(\bar{x}_{m}^{i}\right)+E_{i}\left(\bar{u}_{m}^{i}\right) \mathrm{e} \\
& F_{j}\left(\bar{x}_{m}^{j}\right)=\sum_{s=1}^{m} H_{s j}\left(\bar{x}_{m}^{j}\right)+F_{j}\left(\bar{u}_{m}^{j}\right),
\end{aligned}
$$


onde $u_{k}=\epsilon_{k}\left(x_{k}\right)$. Com efeito,

$$
\begin{aligned}
\sum_{s=1}^{m} G_{s i}\left(\bar{x}_{m}^{i}\right)= & \sum_{s=1}^{m}\left(E_{i}\left(\tilde{x}_{s}^{i}\right)-E_{i}\left(\tilde{x}_{s-1}^{i}\right)\right) \\
= & E_{i}\left(\tilde{x}_{1}^{i}\right)-E_{i}\left(\tilde{x}_{0}^{i}\right)+E_{i}\left(\tilde{x}_{2}^{i}\right)-E_{i}\left(\tilde{x}_{1}^{i}\right)+E_{i}\left(\tilde{x}_{3}^{i}\right)-E_{i}\left(\tilde{x}_{2}^{i}\right)+\ldots \\
& \ldots+E_{i}\left(\tilde{x}_{m-1}^{i}\right)-E_{i}\left(\tilde{x}_{m-2}^{i}\right)+E_{i}\left(\tilde{x}_{m}^{i}\right)-E_{i}\left(\tilde{x}_{m-1}^{i}\right) \\
= & -E_{i}\left(\tilde{x}_{0}^{i}\right)+E_{i}\left(\tilde{x}_{m}^{i}\right) \\
= & E_{i}\left(\bar{x}_{m}^{i}\right)-E_{i}\left(\bar{u}_{m}^{i}\right), \mathrm{e} \\
\sum_{s=1}^{m} H_{s j}\left(\bar{x}_{m}^{j}\right)= & \sum_{s=1}^{m}\left(F_{j}\left(\tilde{x}_{s}^{j}\right)-F_{j}\left(\tilde{x}_{s-1}^{j}\right)\right) \\
= & F_{j}\left(\tilde{x}_{1}^{j}\right)-F_{j}\left(\tilde{x}_{0}^{j}\right)+F_{j}\left(\tilde{x}_{2}^{j}\right)-F_{j}\left(\tilde{x}_{1}^{j}\right)+F_{j}\left(\tilde{x}_{3}^{j}\right)-F_{j}\left(\tilde{x}_{2}^{j}\right)+\ldots \\
& \ldots+F_{j}\left(\tilde{x}_{m-1}^{j}\right)-F_{j}\left(\tilde{x}_{m-2}^{j}\right)+F_{j}\left(\tilde{x}_{m}^{j}\right)-F_{j}\left(\tilde{x}_{m-1}^{j}\right) \\
= & -F_{j}\left(\tilde{x}_{0}^{j}\right)+F_{j}\left(\tilde{x}_{m}^{j}\right) \\
= & F_{j}\left(\bar{x}_{m}^{j}\right)-F_{j}\left(\bar{u}_{m}^{j}\right) .
\end{aligned}
$$

Em vista dessas afirmações, a prova está completa para o caso particular $\mathcal{I} \cup \mathcal{J}=\{1,2, \ldots, m\}$. Note que assumimos o resultado válido para $|\mathcal{I} \cup \mathcal{J}|=m-1$ e o provamos válido para $|\mathcal{I} \cup \mathcal{J}|=m$.

No caso geral, podemos assumir, sem perda de generalidade, $\mathcal{I} \cup \mathcal{J}=\{1,2, \ldots, r\}$, onde $1 \leq$ $r \leq m$. Seja $z \in S_{r+1} \times \ldots \times S_{m}$, em $S_{1} \times \ldots \times S_{r}$. Defina $E_{z i}\left(\bar{x}_{r}^{i}\right)=E_{i}\left(\bar{x}_{r}^{i}, z\right)$, para $i \in \mathcal{I}$, e $F_{z j}\left(\bar{x}_{r}^{j}\right)=F_{j}\left(\bar{x}_{r}^{j}, z\right)$, para $j \in \mathcal{J}$. Claramente,

$$
\sum_{i \in \mathcal{I}} E_{z i}\left(\bar{x}_{r}^{i}\right) \alpha_{i}\left(x_{i}\right)+\sum_{j \in \mathcal{J}} \alpha_{j}\left(x_{j}\right) F_{z j}\left(\bar{x}_{m}^{j}\right)=0
$$

vale em $S_{1} \times \ldots \times S_{r}$. Desse modo, como $R_{1} \times \ldots \times R_{r}$ é $d$-livre, pois $\hat{R}$ é $d$-livre, o caso particular estudado anteriormente se aplica aqui. Com efeito, observe que a I.F. acima depende de $r$ variáveis, e as somas estão indexadas pelos conjuntos $\mathcal{I}$ e $\mathcal{J}$ tais que $|\mathcal{I} \cup \mathcal{J}|=r$. Portanto, existem aplicações $p_{z i j}: \hat{S}_{r}^{i j} \rightarrow Q, i \in \mathcal{I}, j \in \mathcal{J}, i \neq j$, e $\lambda_{z k}: \hat{S}_{r}^{k} \rightarrow Q, k \in \mathcal{I} \cup \mathcal{J}$, tais que

$$
\begin{gathered}
E_{z i}\left(\bar{x}_{r}^{i}\right)=\sum_{\substack{j \in \mathcal{J} \\
j \neq i}} \alpha_{j}\left(x_{j}\right) p_{z i j}\left(\bar{x}_{r}^{i j}\right)+\lambda_{z i}\left(\bar{x}_{r}^{i}\right), i \in \mathcal{I}, \\
F_{z j}\left(\bar{x}_{r}^{j}\right)=-\sum_{\substack{i \in \mathcal{I} \\
i \neq j}} p_{z i j}\left(\bar{x}_{r}^{i j}\right) \alpha_{i}\left(x_{i}\right)-\lambda_{z j}\left(\bar{x}_{r}^{j}\right), j \in \mathcal{J}, \\
\lambda_{z k}=0, \text { se } k \notin \mathcal{I} \cap \mathcal{J} .
\end{gathered}
$$

Finalmente, definindo $p_{i j}\left(\bar{x}_{r}^{i j}, z\right)=p_{z i j}\left(\bar{x}_{r}^{i j}\right)$ e $\lambda_{k}\left(\bar{x}_{r}^{k}, z\right)=\lambda_{z k}\left(\bar{x}_{r}^{k}\right)$, para todo $\left(\bar{x}_{r}, z\right) \in \hat{S}$, a prova está completa.

Dado um subconjunto $R$ d-livre de $Q$, podemos ainda construir novos conjuntos $d$-livres, a saber

Lema 6.1.2. Se $R$ é um subconjunto $d$-livre do anel $Q$, com elemento identidade e centro $C$, e $\delta: R \rightarrow Q$ é uma aplicação arbitrária, então, $\breve{R}=\{(x, \delta(x)) \mid x \in R\}$ é um subconjunto $d$-livre do 
anel $\breve{Q}=Q \times Q$, onde a adição em $\breve{Q}$ é definida coordenada a coordenada, e a multiplicação é dada por

$$
(x, y)(z, w)=(x z, x w+y z)
$$

para $(x, y),(z, w) \in \breve{Q}$.

Demonstração. Vamos provar somente que $\breve{R}$ satisfaz a condição (a) da Definição 6.1.2, pois a condição (b) pode ser provada de maneira análoga. Sejam $\mathcal{I}, \mathcal{J} \subseteq\{1,2, \ldots, m\}$ subconjuntos tais que $\max \{|\mathcal{I}|,|\mathcal{J}|\} \leq d$ e $\breve{E}_{i}, \breve{F}_{j}: \breve{R}^{m-1} \rightarrow \breve{Q}$ funções satisfazendo

$$
\sum_{i \in \mathcal{I}} \breve{E}_{i}\left(\bar{y}_{m}^{i}\right) y_{i}+\sum_{j \in \mathcal{J}} y_{j} \breve{F}_{j}\left(\bar{y}_{m}^{j}\right)=0, \quad \text { para todo } \bar{y}_{m} \in \breve{R}^{m}
$$

onde $y_{i}=\left(x_{i}, \delta\left(x_{i}\right)\right) \in \breve{R}$ e $x_{i} \in R$. Agora, cada aplicação $\breve{E}_{i}$ determina funções $E_{i}, G_{i}: R^{m-1} \rightarrow Q$ tais que

$$
\breve{E}_{i}\left(\bar{y}_{m}^{i}\right)=\left(E_{i}\left(\bar{x}_{m}^{i}\right), G_{i}\left(\bar{x}_{m}^{i}\right)\right)
$$

e cada aplicação $\breve{F}_{j}$ determina funções $F_{j}, H_{j}: R^{m-1} \rightarrow Q$ tais que

$$
\breve{F}_{j}\left(\bar{y}_{m}^{j}\right)=\left(F_{j}\left(\bar{x}_{m}^{j}\right), H_{j}\left(\bar{x}_{m}^{j}\right)\right)
$$

Substituindo estas expressões em (6.16), resulta

$$
\begin{gathered}
\sum_{i \in \mathcal{I}} E_{i}\left(\bar{x}_{m}^{i}\right) x_{i}+\sum_{j \in \mathcal{J}} x_{j} F_{j}\left(\bar{x}_{m}^{j}\right)=0 \mathrm{e} \\
\sum_{i \in \mathcal{I}}\left\{E_{i}\left(\bar{x}_{m}^{i}\right) \delta\left(x_{i}\right)+G_{i}\left(\bar{x}_{m}^{i}\right) x_{i}\right\}+\sum_{j \in \mathcal{J}}\left\{\delta\left(x_{j}\right) F_{j}\left(\bar{x}_{m}^{j}\right)+x_{j} H_{j}\left(\bar{x}_{m}^{j}\right)\right\}=0,
\end{gathered}
$$

para todo $\bar{x}_{m} \in R^{m}$. Assim, como $R$ é $d$-livre, existem aplicações $p_{i j}: R^{m-2} \rightarrow Q$ e $\lambda_{k}: R^{m-1} \rightarrow C$, tais que

$$
\begin{gathered}
E_{i}\left(\bar{x}_{m}^{i}\right)=\sum_{\substack{j \in \mathcal{J} \\
j \neq i}} x_{j} p_{i j}\left(\bar{x}_{m}^{i j}\right)+\lambda_{i}\left(\bar{x}_{m}^{i}\right), \\
F_{j}\left(\bar{x}_{m}^{j}\right)=-\sum_{\substack{i \in \mathcal{I} \\
i \neq j}} p_{i j}\left(\bar{x}_{m}^{i j}\right) x_{i}-\lambda_{j}\left(\bar{x}_{m}^{j}\right), \\
\lambda_{k}=0 \text { se } k \notin \mathcal{I} \cap \mathcal{J} .
\end{gathered}
$$

Desse modo, usando estas últimas igualdades em (6.18), obtemos

$$
\begin{aligned}
& \sum_{i \in \mathcal{I}} \sum_{\substack{j \in \mathcal{J} \\
j \neq i}} x_{j} p_{i j}\left(\bar{x}_{m}^{i j}\right) \delta\left(x_{i}\right)+\sum_{i \in \mathcal{I}} \lambda_{i}\left(\bar{x}_{m}^{i}\right) \delta\left(x_{i}\right)+\sum_{i \in \mathcal{I}} G_{i}\left(\bar{x}_{m}^{i}\right) x_{i} \\
& \quad-\sum_{j \in \mathcal{J}} \sum_{\substack{i \in \mathcal{I} \\
i \neq j}} \delta\left(x_{j}\right) p_{i j}\left(\bar{x}_{m}^{i j}\right) x_{i}-\sum_{j \in \mathcal{J}} \lambda_{j}\left(\bar{x}_{m}^{j}\right) \delta\left(x_{j}\right)+\sum_{j \in \mathcal{J}} x_{j} H_{j}\left(\bar{x}_{m}^{j}\right)=0 .
\end{aligned}
$$

Portanto, como $\lambda_{k}=0$, se $k \notin \mathcal{I} \cap \mathcal{J}$, e $\lambda_{k}\left(\bar{x}_{m}^{k}\right) \in C$, temos

$$
\sum_{i \in \mathcal{I}} \lambda_{i}\left(\bar{x}_{m}^{i}\right) \delta\left(x_{i}\right)-\sum_{j \in \mathcal{J}} \lambda_{j}\left(\bar{x}_{m}^{j}\right) \delta\left(x_{j}\right)=0
$$


e podemos reescrever a igualdade acima como

$$
\sum_{i \in \mathcal{I}}\left(G_{i}\left(\bar{x}_{m}^{i}\right)-\sum_{\substack{j \in \mathcal{J} \\ j \neq i}} \delta\left(x_{j}\right) p_{i j}\left(\bar{x}_{m}^{i j}\right)\right) x_{i}+\sum_{j \in \mathcal{J}} x_{j}\left(H_{j}\left(\bar{x}_{m}^{j}\right)+\sum_{\substack{i \in \mathcal{I} \\ i \neq j}} p_{i j}\left(\bar{x}_{m}^{i j}\right) \delta\left(x_{i}\right)\right)=0 .
$$

Agora, pela $d$-liberdade de $R$, segue que existem aplicações $q_{i j}: R^{m-2} \rightarrow Q$ e $\mu_{k}: R^{m-1} \rightarrow C$ tais que

$$
\begin{gathered}
G_{i}\left(\bar{x}_{m}^{i}\right)-\sum_{\substack{j \in \mathcal{J} \\
j \neq i}} \delta\left(x_{j}\right) p_{i j}\left(\bar{x}_{m}^{i j}\right)=\sum_{\substack{j \in \mathcal{J} \\
j \neq i}} x_{j} q_{i j}\left(\bar{x}_{m}^{i j}\right)+\mu_{i}\left(\bar{x}_{m}^{i}\right), \\
H_{j}\left(\bar{x}_{m}^{j}\right)+\sum_{\substack{i \in \mathcal{I} \\
i \neq j}} p_{i j}\left(\bar{x}_{m}^{i j}\right) \delta\left(x_{i}\right)=-\sum_{\substack{i \in \mathcal{I} \\
i \neq j}} q_{i j}\left(\bar{x}_{m}^{i j}\right) x_{i}-\mu_{j}\left(\bar{x}_{m}^{j}\right), \\
\mu_{k}=0 \text { se } k \notin \mathcal{I} \cap \mathcal{J} .
\end{gathered}
$$

Prosseguindo, defina as aplicações $\breve{p}_{i j}: \breve{R}^{m-2} \rightarrow \breve{Q}$ e $\breve{\lambda}_{k}: \breve{R}^{m-1} \rightarrow \breve{C}$ (o centro de $\breve{Q}$ ) por

$$
\begin{aligned}
\breve{p}_{i j}\left(\bar{y}_{m}^{i j}\right) & =\left(p_{i j}\left(\bar{x}_{m}^{i j}\right), q_{i j}\left(\bar{x}_{m}^{i j}\right)\right), \\
\breve{\lambda}_{k}\left(\bar{y}_{m}^{k}\right) & =\left(\lambda_{k}\left(\bar{x}_{m}^{k}\right), \mu_{k}\left(\bar{x}_{m}^{k}\right)\right) .
\end{aligned}
$$

Observemos que vale $\breve{C}=C \times C$. Claramente, $\breve{\lambda}_{k}=0$ se $k \notin \mathcal{I} \cap \mathcal{J}$. Além disso, temos

$$
\begin{aligned}
\breve{E}_{i}\left(\bar{y}_{m}^{i}\right)= & \left(\sum_{\substack{j \in \mathcal{J} \\
j \neq i}} x_{j} p_{i j}\left(\bar{x}_{m}^{i j}\right)+\lambda_{i}\left(\bar{x}_{m}^{i}\right), \sum_{\substack{j \in \mathcal{J} \\
j \neq i}}\left[\delta\left(x_{j}\right) p_{i j}\left(\bar{x}_{m}^{i j}\right)+x_{j} q_{i j}\left(\bar{x}_{m}^{i j}\right)\right]+\mu_{i}\left(\bar{x}_{m}^{i}\right)\right) \\
= & \sum_{\substack{j \in \mathcal{J} \\
j \neq i}}\left(x_{j}, \delta\left(x_{j}\right)\right)\left(p_{i j}\left(\bar{x}_{m}^{i j}\right), q_{i j}\left(\bar{x}_{m}^{i j}\right)\right)+\left(\lambda_{i}\left(\bar{x}_{m}^{i}\right), \mu_{i}\left(\bar{x}_{m}^{i}\right)\right) \\
= & \sum_{\substack{j \in \mathcal{J} \\
j \neq i}} y_{j} \breve{p}_{i j}\left(\bar{y}_{m}^{i j}\right)+\breve{\lambda}_{i}\left(\bar{y}_{m}^{i}\right) \mathrm{e} \\
\breve{F}_{j}\left(\bar{y}_{m}^{j}\right)= & -\left(\sum_{\substack{i \in \mathcal{I} \\
i \neq j}} p_{i j}\left(\bar{x}_{m}^{i j}\right) x_{i}+\lambda_{j}\left(\bar{x}_{m}^{j}\right), \sum_{\substack{i \in \mathcal{I} \\
i \neq j}}\left[p_{i j}\left(\bar{x}_{m}^{i j}\right) \delta\left(x_{i}\right)+q_{i j}\left(\bar{x}_{m}^{i j}\right) x_{i}\right]+\mu_{j}\left(\bar{x}_{m}^{j}\right)\right) \\
= & -\sum_{\substack{i \in \mathcal{I} \\
i \neq j}}\left(p_{i j}\left(\bar{x}_{m}^{i j}\right), q_{i j}\left(\bar{x}_{m}^{i j}\right)\right)\left(x_{i}, \delta\left(x_{i}\right)\right)-\left(\lambda_{j}\left(\bar{x}_{m}^{j}\right), \mu_{j}\left(\bar{x}_{m}^{j}\right)\right) \\
= & -\sum_{\substack{i \in \mathcal{I} \\
i \neq j}} \breve{p}_{i j}\left(\bar{y}_{m}^{i j}\right) y_{i}-\breve{\lambda}_{j}\left(\bar{y}_{m}^{j}\right) .
\end{aligned}
$$

Logo, a I.F. de (6.16) possui apenas a solução padrão.

Nesse momento, voltaremos a tratar da identidade funcional que iguala uma função de núcleo a uma quasi-polinomial

$$
\sum_{M, N} M B_{M, N} N=\sum_{L} \lambda_{L} L
$$


Nosso objetivo é mostrar que, sob algumas hipóteses, as funções de meio da função de núcleo, também são quasi-polinomiais. Antes de enunciarmos e provarmos o próximo lema, observemos que todo monômio $L$ de grau maior ou igual a 1, pode ser escrito como $L=X_{i} M$, para um certo $i$, com $\operatorname{grau}(M)=\operatorname{grau}(L)-1$. Consequentemente, toda quasi-polinomial $P=\sum_{L} \lambda_{L} L$, de grau $m \geq 1$, pode ser representada por

$$
P=\sum_{i=1}^{m} X_{i} P_{i}+\lambda_{1},
$$

onde cada $P_{i}$ é 0 ou uma quasi-polinomial de grau $m-1$.

Recordemos que estamos na situação em que $S_{1}, \ldots, S_{m}$ são conjuntos não vazios, $\alpha_{i}: S_{i} \rightarrow Q$ aplicações, e $R_{i}=\alpha_{i}\left(S_{i}\right), i=1,2, \ldots, m$. Além disso, $\hat{S}$ é o produto cartesiano dos $S_{i}$ 's, $\hat{R}$ é o produto cartesiano dos $R_{i}$ 's, e $Q$ é um anel com elemento identidade e centro $C$.

Lema 6.1.3. Seja $P=\sum_{L} \lambda_{L} L$ uma quasi-polinomial de grau $\leq m$, e suponha que $\hat{R}$ é $m$-livre e $\lambda_{1}=0$, ou $\hat{R}$ é $(m+1)$-livre. Então, $P=0$ se, e somente se, cada $\lambda_{L}$ é nulo.

Demonstração. Faremos a demonstração por indução em $m$. Se $m=1$, temos $\lambda_{X_{1}} X_{1}=-\lambda_{1}$. Se $\lambda_{1}=0$ e $\hat{R}$ é 1-livre, então, $\lambda_{X_{1}} X_{1}=0$ implica $\lambda_{X_{1}}=0$; e se $\hat{R}$ é 2-livre, então, $\lambda_{X_{1}} X_{1}=-\lambda_{1} \in C$ implica $\lambda_{X_{1}}=0$. Portanto, também vale $\lambda_{1}=0$. Agora, para $m>1$, escrevendo $P$ como em (6.19), vemos $\sum_{i=1}^{m} X_{i} P_{i}=-\lambda_{1}$. Novamente, se $\lambda_{1}=0$ e $\hat{R}$ é $m$-livre, então, $\sum_{i=1}^{m} X_{i} P_{i}=0$ implica $P_{i}=0$; e se $\hat{R}$ é $(m+1)$-livre, então, $\sum_{i=1}^{m} X_{i} P_{i}=-\lambda_{1} \in C$ implica $P_{i}=0$. Logo, $\lambda_{1}=0$. Finalmente, como $\operatorname{grau}\left(P_{i}\right)<m$, aplicando a hipótese de indução, concluímos o resultado.

Lema 6.1.4. Sejam $n, p, r$ inteiros não negativos tais que $n<m$ e $p+n+r=m$. Para cada par $M, N, \operatorname{com} \operatorname{grau}(M)=p$ e $\operatorname{grau}(N)=r$, seja $B_{M, N}: \hat{S}^{M N} \rightarrow Q$. Suponha

$$
\sum_{M, N} M B_{M, N} N=P
$$

onde $P$ é uma quasi-polinomial, com coeficiente central $\lambda_{1}$. Se $\hat{R}$ é $m$-livre e $\lambda_{1}=0$, ou $\hat{R}$ é $(m+1)$-livre, então, cada $B_{M, N}$ é uma quasi-polinomial.

Demonstração. A prova será por indução em $m$. Para $m=1, n=0$, e podemos assumir $p=1$, o que implica $r=0$. Assim, $B_{X_{1}, 1}=a \in Q$ e (6.20) se reduz a $X_{1} a=\lambda_{X_{1}} X_{1}+\lambda_{1}$, ou seja,

$$
X_{1}\left(a-\lambda_{X_{1}}\right)=\lambda_{1},
$$

pois $\lambda_{X_{1}} \in C$ e $\lambda_{1}: S \rightarrow C$. Portanto, se $\lambda_{1}=0$ e $\hat{R}$ é 1-livre, então, $X_{1}\left(a-\lambda_{X_{1}}\right)=0$ implica $a-\lambda_{X_{1}}=0$. Do mesmo modo, se $\hat{R}$ é 2-livre, então, $X_{1}\left(a-\lambda_{X_{1}}\right)=\lambda_{1} \in C$ também implica $a-\lambda_{X_{1}}=0$, e consequentemente, $\lambda_{1}=0$. Desse modo, concluímos que $B_{X_{1}, 1}=a=\lambda_{X_{1}} \in C$ é uma quasi-polinomial. No caso indutivo temos $m>1$, podendo assumir $p>0$. Note que como cada função monomial $M$ pode ser escrita como $M=X_{i} K$, para um $i$ adequado, onde $\operatorname{grau}(K)=p-1$ 
e $K$ não "depende" de $X_{i}$, podemos reescrever (6.20) como

$$
\sum_{i=1}^{m} X_{i}\left(\sum_{K, N} K B_{X_{i} K, N} N\right)=\sum_{i=1}^{m} X_{i} P_{i}+\lambda_{1}
$$

onde $P_{i}$ é uma quasi-polinomial e $\lambda_{1}: \hat{S} \rightarrow C$. Agora, se $\lambda_{1}=0$ e $\hat{R}$ é $m$-livre, então,

$$
\sum_{i=1}^{m} X_{i}\left(\sum_{K, N} K B_{X_{i} K, N} N-P_{i}\right)=0
$$

implica $\sum_{K, N} K B_{X_{i} K, N} N=P_{i}$, para cada $i=1,2, \ldots, m$. Analogamente, se $\hat{R}$ é $(m+1)$-livre, então, $\sum_{i=1}^{m} X_{i}\left(\sum_{K, N} K B_{X_{i} K, N} N-P_{i}\right)=\lambda_{1} \in C$ implica $\sum_{K, N} K B_{X_{i} K, N} N=P_{i}$, para cada $i=1,2, \ldots, m$. Portanto, como esta identidade não envolve $X_{i}$, podemos aplicar a hipótese de indução, obtendo que cada $B_{M, N}=B_{X_{i} K, N}$ é uma quasi-polinomial.

Lema 6.1.5. Seja $0 \leq n \leq m$, e para cada monômio $M$, com grau $(M)=m-n$, seja $B_{M}: \hat{S}^{M} \rightarrow Q$. Suponha

$$
\sum_{M} M B_{M}=\sum_{i=1}^{m} F_{i} X_{i}+\lambda
$$

onde $F_{i}: \hat{S}^{X_{i}} \rightarrow Q$ e $\lambda: \hat{S} \rightarrow C$. Se $\hat{R}$ é $m$-livre e $\lambda=0$, ou $\hat{R}$ é $(m+1)$-livre, então, cada $B_{M}$ é da forma

$$
B_{M}=\sum_{i \notin \operatorname{dom}(M)} p_{i M} X_{i}+\mu_{M}
$$

onde $p_{i M}: \hat{S}^{M X_{i}} \rightarrow Q$ e $\mu_{M}: \hat{S}^{M} \rightarrow C$.

Demonstração. A prova será por indução em $\operatorname{grau}(M)=m-n$. Se grau $(M)=0$, não há nada a ser feito. Suponha $\operatorname{grau}(M)>0$ e reescreva (6.21) como

$$
\sum_{j=1}^{m} X_{j}\left(\sum_{K} K B_{X_{j} K}\right)-\sum_{i=1}^{m} F_{i} X_{i}=\lambda
$$

onde $\operatorname{grau}(K)=\operatorname{grau}(M)-1$. Se $\hat{R}$ é $m$-livre e $\lambda=0$ ou $\hat{R}$ é $(m+1)$-livre, segue

$$
\sum_{K} K B_{X_{j} K}=\sum_{\substack{i=1 \\ i \neq j}}^{m} p_{i j} X_{i}+\mu_{j}
$$

onde $p_{i j}: \hat{S}^{X_{i} X_{j}} \rightarrow Q$ e $\mu_{j}: \hat{S}^{X_{j}} \rightarrow C$. Assim, aplicando a hipótese de indução, concluímos que cada $B_{M}=B_{X_{j} K}$ tem a forma desejada.

Teorema 6.1.2. (Brešar, Chebotar e Martindale, 3rd, [8]) Seja $0 \leq n<m$, e para cada par $M, N$ de monômios, tais que grau $(M N)=m-n$, seja $B_{M, N}: \hat{S}^{M N} \rightarrow Q$. Suponha

$$
\sum_{M, N} M B_{M, N} N=P
$$


onde $P$ é uma quasi-polinomial com coeficiente central $\lambda_{1}$. Se $\hat{R}$ é $m$-livre e $\lambda_{1}=0$, ou $\hat{R}$ é $(m+1)$ livre, então, para cada $M, N$,

$$
B_{M, N}=\sum_{i \notin \operatorname{dom}(M N)}\left(p_{M, N, X_{i}} X_{i}+X_{i} q_{X_{i}, M, N}\right)+\mu_{M, N}
$$

onde $p_{M, N, X_{i}}, q_{X_{i}, M, N}: \hat{S}^{M N X_{i}} \rightarrow Q$ e $\mu_{M, N}: \hat{S}^{M N} \rightarrow C$. Além disso, se $B_{M, N}$ é uma função de meio mais à direita (respectivamente, função de meio mais à esquerda), então,

$$
\begin{gathered}
q_{X_{i}, M, N}=0 \text { para todo } M \text { e } i \\
\text { (respectivamente, } p_{M, N, X_{i}}=0 \text { para todo } N \text { e } i \text { ). }
\end{gathered}
$$

Demonstração. A demonstração será por indução em $m$. Para $m=1$, temos $n=0$. Desse modo, (6.22) se reduz a

$$
X_{1} a+b X_{1}=\lambda_{X_{1}} X_{1}+\lambda_{1},
$$

onde $a, b \in Q, \lambda_{X_{1}} \in C$ e $\lambda_{1}: S_{1} \rightarrow C$. Rearranjando os termos, chegamos a

$$
X_{1} a+\left(b-\lambda_{X_{1}}\right) X_{1}=\lambda_{1}
$$

Em decorrência das hipóteses de " $d$-liberdade", concluímos $a, b-\lambda_{X_{1}} \in C$. Portanto, $a, b \in C$.

Seja $m>1$, considere $v$ o grau mínimo entre os graus dos monômios $N$, tais que $B_{M, N} \neq 0$ e suponha que $v$ é um inteiro positivo. Desse modo, como cada $N$ em (6.22) é tal que $N=K L$, onde $\operatorname{grau}(L)=v$, podemos reescrever (6.22) na forma

$$
\sum_{\operatorname{grau}(L)=v}\left[\sum_{M, K} M B_{M, K L} K\right] L=P .
$$

Logo, pelo Lema (6.1.4), concluímos que para cada $L$, com grau $(L)=v$, temos que

$$
\sum_{M, K} M D_{M, K} K \quad\left(D_{M, K}=B_{M, K L}\right)
$$

é uma quasi-polinomial. Assim, como (6.25) só depende das variáveis de $\hat{S}^{L}$ (desse modo, $m$ é substituído por $m-v$ ), pela hipótese de indução, concluímos que cada $B_{M, K L}=D_{M, K}$ é da forma requerida em (6.23). Agora, suponha $B_{M, L} \neq 0$, para algum $M$, ou seja, $B_{M, L}$ é função de meio mais à direita. Assim, $D_{M, 1} \neq 0$ para essa escolha de $M\left(D_{M, 1}\right.$ é também função de meio mais à direita), e pela hipótese de indução aplicada a (6.25), vemos que $D_{M, 1}$, e portanto $B_{M, L}$, tem a forma requerida em (6.24). Portanto, (6.24) foi provada para $N$ de grau mínimo $v$, e por simetria, segue que (6.24) também está provada para $M$ de grau mínimo $u$. Como resultado disso, podemos assumir $v=0$, e similarmente podemos assumir $u=0$.

Em vista de que vale $N=U X_{i}$, se $\operatorname{grau}(N)>0$, e $M=X_{j} V$, se $\operatorname{grau}(M)>0$, reescrevemos (6.22) como

$$
\sum_{i=1}^{m}\left[\sum_{M, U} M B_{M, U X_{i}} U\right] X_{i}+\sum_{j=1}^{m} X_{j}\left[\sum_{V} V B_{X_{j} V, 1}\right]=\sum_{i=1}^{m} P_{i} X_{i}+\lambda_{1}
$$


onde cada $P_{i}$ é uma quasi-polinomial. Pelas condições de " $d$-liberdade", vale que, para cada $j$,

$$
\sum_{V} V B_{X_{j} V, 1}=\sum_{\substack{i=1 \\ i \neq j}}^{m} r_{i j} X_{i}+\mu_{j}
$$

onde $r_{i j}: \hat{S}^{X_{i} X_{j}} \rightarrow Q$ e $\mu_{j}: \hat{S}^{X_{j}} \rightarrow C$. Assim, pelo Lema 6.1.5, vemos

$$
B_{M, 1}=B_{X_{j} V, 1}=\sum_{i \notin \operatorname{dom}(M)} p_{M, X_{i}} X_{i}+\gamma_{M}
$$

onde $p_{M, X_{i}}: \hat{S}^{M X_{i}} \rightarrow Q$ e $\gamma_{M}: \hat{S}^{M} \rightarrow C$. Por simetria, pode-se provar

$$
B_{1, N}=\sum_{j \notin \operatorname{dom}(N)} X_{j} q_{X_{j}, N}+\gamma_{N}
$$

Novamente voltamos a (6.22), desta vez reescrevendo-a como

$$
\sum_{\substack{M, N \\ \operatorname{grau}(N)>0}} M B_{M, N} N+\sum_{W} W B_{W, 1}
$$

sendo uma quasi-polinomial. Escrevendo $N=U X_{i}, W=M X_{j}$ e usando (6.26), temos que (6.27) se traduz como

$$
\begin{array}{r}
\sum_{i=1}^{m}\left[\sum_{\substack{M, U \\
\operatorname{grau}(U)>0}} M B_{M, U X_{i}} U\right] X_{i}+\sum_{i=1}^{m}\left[\sum _ { \operatorname { g r a u } ( M ) = m - n - 1 } M \left(B_{M, X_{i}}\right.\right. \\
\left.\left.+\sum_{j \notin \operatorname{dom}\left(M X_{i}\right)} X_{j} p_{M, X_{j}, X_{i}}\right)\right] X_{i}
\end{array}
$$

é uma quasi-polinomial, para algumas funções $p_{M, X_{j}, X_{i}}$. Pelo Lema 6.1.4, segue que, para cada $i$,

$$
\sum_{\substack{M, U \\ \operatorname{grau}(U)>0}} M B_{M, U X_{i}} U+\sum_{\operatorname{grau}(M)=m-n-1} M\left(B_{M, X_{i}}+\sum_{j \notin \operatorname{dom}\left(M X_{i}\right)} X_{j} p_{M, X_{j}, X_{i}}\right)
$$

é uma quasi-polinomial. Portanto, como esta relação somente depende das variáveis de $\hat{S}^{X_{i}}$ (desse modo, $m$ é substituído por $m-1$ ) concluímos da hipótese de indução, que $B_{M, U X_{i}}$ é da forma requerida em (6.23), se $\operatorname{grau}(U)>0$, e

$$
B_{M, X_{i}}+\sum_{j \notin \operatorname{dom}\left(M X_{i}\right)} X_{j} p_{M, X_{j}, X_{i}}=\sum_{k \notin \operatorname{dom}\left(M X_{i}\right)} q_{X_{k}, M, X_{i}} X_{k}+\mu_{M, X_{i}}
$$

Logo, $B_{M, X_{i}}$ é também da forma requerida em (6.23).

A fim de esclarecer um pouco mais a tese do teorema anterior, vamos enunciá-lo nos casos $n=0$ ou $n=1$. 
Lema 6.1.6. Nas hipóteses do Teorema 6.1.2, temos que as seguintes afirmações são válidas:

(i) Se $n=0$, então, cada $B_{M, N}$ pertence a $C$;

(ii) Se $n=1$, então, para cada $M, N$, existem aplicações $\mu_{M, N}: S_{i} \rightarrow C$, onde $X_{i}$ não é um fator de $M N$, e elementos $b_{M, N}, c_{M, N} \in Q$, tais que

$$
B_{M, N}=b_{M, N} X_{i}+X_{i} c_{M, N}+\mu_{M, N}
$$

Vamos considerar agora o caso $S_{1}=S_{2}=\ldots=S_{m}=S, R_{1}=R_{2}=\ldots=R_{m}=R$ e $\alpha_{1}=\alpha_{2}=\ldots=\alpha_{m}=\alpha$. Uma das importantes hipóteses adicionais que faremos no próximo resultado é que cada função de meio $B_{M, N}$ é, a menos de uma constante de $C$, igual a uma função de meio mais à esquerda $B_{U, V}$, para que seja possível usar a conclusão (6.24) do Teorema 6.1.2. Enfatizamos que, com a notação $B_{M, N}=c B_{U, V}, c \in C$, queremos dizer

$$
B_{M, N}\left(x_{1}, x_{2}, \ldots, x_{n}\right)=c B_{U, V}\left(x_{\pi(1)}, x_{\pi(2)}, \ldots, x_{\pi(n)}\right)
$$

para todos $x_{1}, x_{2}, \ldots, x_{n} \in S$, e para alguma permutação $\pi \in S_{n}$. Além disso, $n$ é a aridade das funções de meio.

Teorema 6.1.3. (Brešar, Chebotar e Martindale, 3rd, [8]) Seja $0 \leq n<m$, e para cada par de monômios $M, N, \operatorname{com} \operatorname{grau}(M N)=m-n$, seja $B_{M, N}: S^{n} \rightarrow Q$. Suponha

$$
\sum_{M, N} M B_{M, N} N=P
$$

onde $P$ é uma quasi-polinomial com coeficiente central $\lambda_{1}$. Se $R$ é $m$-livre e $\lambda_{1}=0$, ou $R$ é $(m+1)$ livre, e para toda função de meio $B_{M, N}$, existe uma função de meio mais à esquerda $B_{U, V}$ e um elemento $c \in C$, tais que $B_{M, N}=c B_{U, V}$, então, todas as $B_{M, N}$ 's são quasi-polinomiais.

Demonstração. Procederemos por indução em $n$. O caso $n=0$ é dado por (i) do Lema 6.1.6. Seja $n>0$. Pelo Teorema 6.1.2, toda função de meio mais à esquerda $B_{U, V}$ e, pela nossa hipótese, também toda função de meio $B_{M, N}$, é da forma

$$
B_{M, N}=\sum_{i \notin \operatorname{dom}(M N)} X_{i} q_{X_{i}, M, N}+\mu_{M, N}
$$

onde $q_{X_{i}, M, N}=c q_{X_{j}, U, V}$, para algum $j$. Substituindo (6.29) em (6.28), produzimos uma nova I.F., na qual as funções de meio da função de núcleo, são as $q_{X_{i}, M, N}$ 's, e os termos $\mu_{M, N} M N\left(\mu_{M, N} \in C\right)$ são incorporados pela quasi-polinomial original. Assim, como $n$ foi agora substituído por $n-1$, a aridade das $q_{X_{i}, M, N}$ 's, e as hipóteses restantes do teorema ainda valem (note que o grau mínimo da funções mais à esquerda $u$ é $u+1$ ), podemos aplicar a hipótese de indução e concluir que cada $q_{X_{i}, M, N}$ é uma quasi-polinomial. Portanto, (6.29) nos mostra que $B_{M, N}$ também é uma quasi-polinomial.

Como consequência deste último teorema, temos o caso em que, a menos de uma constante em $C$, as $B_{M, N}$ 's são todas iguais a uma mesma função $B$. 
Corolário 6.1.1. Sejam $0 \leq n<m, B: S^{n} \rightarrow Q$ e para cada par de monômios $M, N$, com $\operatorname{grau}(M N)=m-n$, considere $c_{M, N} \in C$. Suponha

$$
\sum_{M, N} c_{M, N} M B N=P
$$

onde $P$ é uma quasi-polinomial com coeficiente central $\lambda_{1}$. Se $R$ é $m$-livre e $\lambda_{1}=0$, ou $R$ é $(m+1)$ livre, e além disso, se pelo menos um $c_{M, N}, \operatorname{com} B$ função de meio mais à esquerda, isto é, $M$ tem grau mínimo entre os graus dos monômios que multiplicam $B$ à esquerda, é invertível em $C$, então, $B$ é uma quasi-polinomial.

Demonstração. Se $c_{U, V}$ é invertível, onde $B_{U, V}=B$ é uma função de meio mais à esquerda, então, para cada $M, N$, temos

$$
c_{M, N} B=c_{M, N} c_{U, V}^{-1} c_{U, V} B
$$

Logo, pelo Teorema 6.1.3, concluímos o resultado.

A partir de agora, nosso objetivo é aplicar a teoria de identidades funcionais em anéis. Para isso, enunciaremos e provaremos dois resultados relacionados a problemas já estudados na dissertação, com as hipóteses convenientes.

No próximo resultado, soma direta tem um sentido diferente do que utilizamos anteriormente na dissertação. A aplicação $\alpha$ é a soma direta de um homomorfismo e um antihomomorfismo, se existir um idempotente central $\epsilon \in Q$ tal que $x \mapsto \alpha(x), x \in A$, é um homomorfismo e $x \mapsto$ $(1-\epsilon) \alpha(x), x \in A$, é um antihomomorfismo.

Teorema 6.1.4. (Brešar, Chebotar e Martindale, 3rd, [8]) Sejam $A$ um anel qualquer e $Q$ um anel com identidade e centro $C$, tal que $\frac{1}{2} \in Q$. Se $\alpha: A \rightarrow Q$ é um homomorfismo de Jordan tal que $\alpha(A)$ é um subconjunto 4-livre de $Q$, então, $\alpha$ é a soma direta de um homomorfismo e um antihomomorfismo.

Demonstração. É fácil ver que vale

$$
[[s, t], u]=s \circ(t \circ u)-t \circ(s \circ u)
$$

para todos $s, t, u \in A$. Assim, como $\alpha$ é um homomorfismo de Jordan, segue

$$
\begin{aligned}
\alpha([[s, t], u]) & =\alpha(s) \circ(\alpha(t) \circ \alpha(u))-\alpha(t) \circ(\alpha(s) \circ \alpha(u)) \\
& =[[\alpha(s), \alpha(t)], \alpha(u)],
\end{aligned}
$$

para todos $s, t, u \in A$. Além disso, temos

$$
\begin{aligned}
{[[\alpha(x), \alpha(y)], \alpha([z, w])] } & =\alpha([[x, y],[z, w]])=-\alpha([[z, w],[x, y]]) \\
& =-[[\alpha(z), \alpha(w)], \alpha([x, y])]=[\alpha([x, y]),[\alpha(z), \alpha(w)]]
\end{aligned}
$$

para quaisquer $x, y, z, w \in A$, o que podemos reescrever como

$$
[[\alpha(x), \alpha(y)], B(z, w)]=[B(x, y),[\alpha(z), \alpha(w)]],
$$


para todos $x, y, z, w \in A$, onde $B(x, y)=\alpha([x, y])$. Observe que (6.30) fornece uma I.F. que iguala uma função de núcleo a uma quasi-polinomial (nula), com coeficiente central igual a 0. Desse modo, como $\alpha(A)$ é um subconjunto 4-livre, é fácil ver que as hipóteses do Teorema 6.1.3 são satisfeitas. Consequentemente, concluímos que $B$ é uma quasi-polinomial. Assim, existem $\lambda, \lambda^{\prime} \in C$, $\mu_{1}, \mu_{2}: A \rightarrow C$ e $\gamma: A^{2} \rightarrow C$, tais que

$$
B(x, y)=\lambda \alpha(x) \alpha(y)+\lambda^{\prime} \alpha(y) \alpha(x)+\mu_{1}(x) \alpha(y)+\mu_{2}(y) \alpha(x)+\gamma(x, y),
$$

para todos $x, y \in A$. Agora, substituindo esta última igualdade em (6.30) e expandindo-a, obtemos

$$
\begin{array}{r}
\left(\lambda+\lambda^{\prime}\right) \alpha(x) \alpha(y) \alpha(z) \alpha(w)+\left(\lambda+\lambda^{\prime}\right) \alpha(z) \alpha(w) \alpha(y) \alpha(x)-\left(\lambda+\lambda^{\prime}\right) \alpha(y) \alpha(x) \alpha(z) \alpha(w) \\
-\left(\lambda+\lambda^{\prime}\right) \alpha(w) \alpha(z) \alpha(x) \alpha(y)+\mu_{1}(z)[[\alpha(x), \alpha(y)], \alpha(w)]+\mu_{2}(w)[[\alpha(x), \alpha(y)], \alpha(z)] \\
-\mu_{1}(x)[\alpha(y),[\alpha(z), \alpha(w)]]-\mu_{2}(y)[\alpha(x),[\alpha(z), \alpha(w)]]=0,
\end{array}
$$

de onde segue $\lambda+\lambda^{\prime}=0$ e $\mu_{1}=\mu_{2}=0$, pelo Lema 6.1.3, pois $\alpha(A)$ é 4-livre. Desse modo, $B(x, y)=\alpha([x, y])=\lambda[\alpha(x), \alpha(y)]+\gamma(x, y)$, ou seja,

$$
\alpha(x y)=\lambda \alpha(x) \alpha(y)-\lambda \alpha(y) \alpha(x)+\alpha(y x)+\gamma(x, y) .
$$

Além disso, $\alpha(x \circ y)=\alpha(x) \circ \alpha(y)$ implica

$$
\alpha(x y)=\alpha(x) \alpha(y)+\alpha(y) \alpha(x)-\alpha(y x)
$$

Comparando estas duas últimas igualdades, chegamos a

$$
\alpha(x y)=\epsilon \alpha(x y)+\epsilon^{\prime} \alpha(y x)+\frac{1}{2} \gamma(x, y),
$$

para todos $x, y \in A$, onde $\epsilon=\frac{1}{2}(1+\lambda)$ e $\epsilon^{\prime}=\frac{1}{2}(1-\lambda)$. Observe que vale $\epsilon+\epsilon^{\prime}=1$ e $\lambda \in C$ implica que $\epsilon$ e $\epsilon^{\prime}$ também pertencem a $C$. Vamos calcular agora, de duas maneiras diferentes, o elemento $\alpha(x y z), x, y, z \in A$. Por um lado, temos

$$
\begin{aligned}
\alpha((x y) z)= & \epsilon \alpha(x y) \alpha(z)+\epsilon^{\prime} \alpha(z) \alpha(x y)+\gamma(x y, z) \\
= & \epsilon^{2} \alpha(x) \alpha(y) \alpha(z)+\epsilon \epsilon^{\prime} \alpha(y) \alpha(x) \alpha(z)+\epsilon \gamma(x, y) \alpha(z)+\epsilon \epsilon^{\prime} \alpha(z) \alpha(x) \alpha(y) \\
& +\epsilon^{\prime 2} \alpha(z) \alpha(y) \alpha(x)+\epsilon^{\prime} \gamma(x, y) \alpha(z)+\gamma(x y, z),
\end{aligned}
$$

e por outro lado, segue

$$
\begin{aligned}
\alpha(x(y z))= & \epsilon \alpha(x) \alpha(y z)+\epsilon^{\prime} \alpha(y z) \alpha(x)+\gamma(x, y z) \\
= & \epsilon^{2} \alpha(x) \alpha(y) \alpha(z)+\epsilon \epsilon^{\prime} \alpha(x) \alpha(z) \alpha(y)+\epsilon \gamma(y, z) \alpha(x)+\epsilon \epsilon^{\prime} \alpha(y) \alpha(z) \alpha(x) \\
& +\epsilon^{\prime 2} \alpha(z) \alpha(y) \alpha(x)+\epsilon^{\prime} \gamma(y, z) \alpha(x)+\gamma(x, y z) .
\end{aligned}
$$

Comparando as duas expressões encontradas, obtemos

$$
\epsilon \epsilon^{\prime}[\alpha(y),[\alpha(x), \alpha(z)]]+\gamma(x, y) \alpha(z)-\gamma(y, z) \alpha(x) \in C .
$$

Esta última pertinência depende de 3 variáveis, e nos leva a uma I.F. que iguala uma quasi- 
polinomial a 0 . Assim, como $\alpha(A)$ é um subconjunto 4-livre, pelo Lema 6.1.3, concluímos $\epsilon \epsilon^{\prime}=0$ e $\gamma=0$. Consequentemente,

$$
\alpha(x y)=\epsilon \alpha(x) \alpha(y)+\epsilon^{\prime} \alpha(y) \alpha(x)
$$

para todos $x, y \in A$. Além disso, de $\epsilon+\epsilon^{\prime}=1$ e $\epsilon \epsilon^{\prime}=0$, obtemos

$$
\epsilon=\epsilon\left(\epsilon+\epsilon^{\prime}\right)=\epsilon^{2}
$$

ou seja, $\epsilon$ é um idempotente central. Finalmente, as igualdades

$$
\begin{aligned}
\epsilon \alpha(x y) & =\epsilon\left(\epsilon \alpha(x) \alpha(y)+\epsilon^{\prime} \alpha(y) \alpha(x)\right) \\
& =(\epsilon \alpha(x))(\epsilon \alpha(y)) \mathrm{e} \\
\epsilon^{\prime} \alpha(x y) & =\epsilon^{\prime}\left(\epsilon \alpha(x) \alpha(y)+\epsilon^{\prime} \alpha(y) \alpha(x)\right) \\
& =\left(\epsilon^{\prime} \alpha(y)\right)\left(\epsilon^{\prime} \alpha(x)\right)
\end{aligned}
$$

nos mostram que $x \mapsto \epsilon \alpha(x)$ é um homomorfismo, e $x \mapsto(1-\epsilon) \alpha(x)$ é um antihomomorfismo.

Corolário 6.1.2. Seja $Q$ um anel com identidade e centro $C$, tal que $\frac{1}{2} \in Q$. Se $A$ é um subanel 4-livre de $Q$, então, toda derivação de Jordan $\delta: A \rightarrow Q$ é uma derivação.

Demonstração. Seja $\delta: A \rightarrow Q$ uma derivação de Jordan. Defina $\breve{Q}=Q \times Q$ como no Lema 6.1.2 e $\alpha: A \rightarrow \breve{Q}$ por $\alpha(x)=(x, \delta(x))$, para todo $x \in A$. A aplicação $\alpha$ claramente é aditiva e

$$
\begin{aligned}
\alpha(x \circ y) & =(x \circ y, \delta(x) \circ y+x \circ \delta(y)) \\
& =(x, \delta(x))(y, \delta(y))+(y, \delta(y))(x, \delta(x)) \\
& =\alpha(x) \circ \alpha(y),
\end{aligned}
$$

para todos $x, y \in A$, isto é, $\alpha$ é um homomorfismo de Jordan. Assim, como $A$ é 4-livre em $Q$, pelo Lema 6.1.2, $\alpha(A)$ é 4-livre em $\breve{Q}$. Desse modo, podemos aplicar o teorema anterior e concluir que $\alpha$ é a soma direta de um homomorfismo e um antihomomorfismo. Suponha que $\epsilon^{\prime}$ é um idempotente central em $\breve{Q}$, tal que $x \mapsto \epsilon^{\prime} \alpha(x)$ é um antihomomorfismo. Denotando $\epsilon^{\prime}=(a, b) \in \breve{Q}$, temos $(a, b)(c, d)=(c, d)(a, b)$, isto é, $(a c, a d+b c)=(c a, c b+d a)$, para todo $(c, d) \in \breve{Q}$. Logo, $a \in C$. Agora, para $(c, d)=(a, b)$, temos $\left(a^{2}, 2 a b\right)=(a, b)$, o que implica que $a$ é idempotente e $2 a b=b$. Consequentemente, $2 a^{2} b=a b$ e, então, $a b=0$. Portanto, $b=0$ e temos $\epsilon^{\prime}=(\omega, 0)$, onde $\omega$ é um idempotente central de $Q$. Finalmente, pela igualdade $\epsilon^{\prime} \alpha(x y)=\epsilon^{\prime} \alpha(y) \alpha(x)$, inferimos em particular, $\omega x y=\omega y x$, para todos $x, y \in A$. Logo, como $A$ é 4-livre, e portanto é 2-livre, segue pelo Lema 6.1.3, que vale $\omega=0$. Consequentemente, $\epsilon^{\prime}=0$, o que mostra que necessariamente $\alpha$ é um homomorfismo e nos permite concluir que $\delta$ é uma derivação.

\subsection{A teoria de identidades funcionais em superálgebras}

Nesta seção, apresentamos uma breve generalização da teoria da seção anterior, para o contexto das superálgebras, a qual é dada por Yu Wang em [32]. Uma vez que estamos interessados nas propriedades de $d$-superliberdade e suas relações com as superquasi-polinomiais, estudamos apenas a generalização de um quadro mais geral das identidades funcionais. 
Seja $\mathcal{Q}=\mathcal{Q}_{0} \oplus \mathcal{Q}_{1}$ uma superálgebra com identidade e centro $\mathcal{C}=\mathcal{C}_{0} \oplus \mathcal{C}_{1}$. Recordamos que o centro de uma superálgebra é uma subálgebra graduada. Além disso, seja $\sigma$ o automorfismo "graduante" associado à $\mathbb{Z}_{2}$-graduação de $\mathcal{Q}$. Denotamos por $\mathcal{C}_{0}^{*}$ o grupo dos elementos invertíveis de $\mathcal{C}_{0}$. Vamos definir um elemento $\omega \in \mathcal{Q}$, da seguinte forma: se $\sigma=\mathrm{id}_{\mathcal{Q}}$ ou $\sigma$ é um automorfismo externo, então, $\omega=0$. Caso contrário, $\omega$ denotará um elemento invertível em $\mathcal{Q}$, tal que $\sigma(a)=$ $\omega a \omega^{-1}$, para todo $a \in \mathcal{Q}$. Vejamos algumas propriedades de $\omega$.

(1) $\omega \in \mathcal{Q}_{0}$, pois $\omega=0 \in \mathcal{Q}_{0}$ ou

$$
\omega_{0}+\omega_{1}=\omega=\omega \omega \omega^{-1}=\sigma(\omega)=\omega_{0}-\omega_{1},
$$

pela definição de $\sigma$, o que implica $\omega_{1}=0$;

(2) $\omega^{2} \in \mathcal{C}_{0}=\mathcal{C} \cap \mathcal{Q}_{0}$, pois como $\omega \in \mathcal{Q}_{0}$, então, também $\omega^{2} \in \mathcal{Q}_{0}$, e para cada $a \in \mathcal{Q}$, temos

$$
\omega^{2} a=\omega\left(\omega a \omega^{-1}\right) \omega=\omega \sigma(a) \omega^{-1} \omega^{2}=\sigma(\sigma(a)) \omega^{2}=a \omega^{2},
$$

pois $\sigma^{2}=\operatorname{id}_{\mathcal{Q}}$

(3) $\omega a_{0}=\sigma\left(a_{0}\right) \omega=a_{0} \omega$, para cada $a_{0} \in \mathcal{Q}_{0}$;

(4) $\omega a_{1}=\sigma\left(a_{1}\right) \omega=-a_{1} \omega$, para todo $a_{1} \in \mathcal{Q}_{1}$.

O elemento $\omega$ será chamado de elemento graduante de $\mathcal{Q}$.

Seja $m$ um inteiro positivo. Para cada $1 \leq i \leq m$, seja $\mathcal{U}_{i}$ um subconjunto de $\mathcal{Q}$, tal que $\mathcal{U}_{i} \subseteq \mathcal{Q}_{0}$ ou $\mathcal{U}_{i} \subseteq \mathcal{Q}_{1}$. Fixamos, para cada $i, \epsilon_{i}=1$, se $\mathcal{U}_{i} \subseteq \mathcal{Q}_{0}$, ou $\epsilon_{i}=-1$, se $\mathcal{U}_{i} \subseteq \mathcal{Q}_{1}$. Sejam $S_{1}, S_{2}, \ldots, S_{m}$ conjuntos não vazios. Para $\mathcal{I}, \mathcal{J} \subseteq\{1,2, \ldots, m\}, \delta_{i}: S_{i} \rightarrow \mathcal{U}_{i}, i \in \mathcal{I} \cup \mathcal{J}$, são aplicações sobrejetivas. Sejam $\hat{S}=\prod_{k=1}^{m} S_{k}, \hat{\mathcal{U}}=\prod_{k=1}^{m} \mathcal{U}_{k}$ e $\Delta=\left\{\delta_{l} \mid l \in \mathcal{I} \cup \mathcal{J}\right\}$.

Para cada $1 \leq i \leq m$ e $G: \prod_{k \neq i} S_{k} \rightarrow \mathcal{Q}$, definimos

$$
G^{i}\left(\bar{x}_{m}\right)=G\left(x_{1}, \ldots, x_{i-1}, x_{i+1}, \ldots, x_{m}\right),
$$

para todo $\bar{x}_{m} \in \hat{S}$. Analogamente, para cada $1 \leq i<j \leq m$ e $H: \prod_{k \neq i, j} S_{k} \rightarrow \mathcal{Q}$, definimos

$$
H^{i j}\left(\bar{x}_{m}\right)=H\left(x_{1}, \ldots, x_{i-1}, x_{i+1}, \ldots, x_{j-1}, x_{j+1}, \ldots, x_{m}\right),
$$

para todo $\bar{x}_{m} \in \hat{S}$, e $H^{j i}=H^{i j}$. Consideraremos identidades funcionais em $\hat{S}$ dos tipos

$$
\begin{gathered}
\sum_{i \in \mathcal{I}} E_{i}^{i}\left(\bar{x}_{m}\right) \delta_{i}\left(x_{i}\right)+\sum_{j \in \mathcal{J}} \delta_{j}\left(x_{j}\right) F_{j}^{j}\left(\bar{x}_{m}\right)=0, \\
\sum_{i \in \mathcal{I}} E_{i}^{i}\left(\bar{x}_{m}\right) \delta_{i}\left(x_{i}\right)+\sum_{j \in \mathcal{J}} \delta_{j}\left(x_{j}\right) F_{j}^{j}\left(\bar{x}_{m}\right) \in \mathcal{C}+\mathcal{C} \omega,
\end{gathered}
$$

para todo $\bar{x}_{m} \in \hat{S}$. Com o intuito de simplificar a notação, abreviaremos as I.F.'s mencionadas como 
segue,

$$
\begin{gathered}
\sum_{i \in \mathcal{I}} E_{i}^{i} \delta_{i}+\sum_{j \in \mathcal{J}} \delta_{j} F_{j}^{j}=0, \\
\sum_{i \in \mathcal{I}} E_{i}^{i} \delta_{i}+\sum_{j \in \mathcal{J}} \delta_{j} F_{j}^{j} \in \mathcal{C}+\mathcal{C} \omega .
\end{gathered}
$$

Pelo que foi feito na seção anterior, podemos definir soluções padrão para essas identidades e é o que faremos a seguir.

Suponha $\omega=0$ ou cada $\mathcal{U}_{i} \subseteq \mathcal{Q}_{0}$. Definindo

$$
\begin{gathered}
E_{i}^{i}=\sum_{\substack{j \in \mathcal{J} \\
j \neq i}} \delta_{j} p_{i j}^{i j}+\lambda_{i}^{i}, \\
F_{j}^{j}=-\sum_{\substack{i \in \mathcal{I} \\
i \neq j}} p_{i j}^{i j} \delta_{i}-\lambda_{j}^{j},
\end{gathered}
$$

onde $p_{i j}: \prod_{k \neq i, j} S_{k} \rightarrow \mathcal{Q}, i \in \mathcal{I}, j \in \mathcal{J}, i \neq j, \lambda_{l}: \prod_{k \neq l} S_{k} \rightarrow \mathcal{C}+\mathcal{C} \omega, l \in \mathcal{I} \cup \mathcal{J}$, e $\lambda_{l}=0$ se $l \notin \mathcal{I} \cap \mathcal{J}$, temos que as I.F.'s são satisfeitas.

Agora, suponha que acontece o caso contrário, isto é, $\omega \neq 0$ e pelo menos um dos $\mathcal{U}_{i}$ 's está contido em $\mathcal{Q}_{1}$. Definindo

$$
\begin{gathered}
E_{i}^{i}=\sum_{\substack{j \in \mathcal{J} \\
j \neq i}} \delta_{j} p_{i j}^{i j}+\lambda_{i}^{i}+\mu_{i}^{i} \omega, \\
F_{j}^{j}=-\sum_{\substack{i \in \mathcal{I} \\
i \neq j}} p_{i j}^{i j} \delta_{i}-\lambda_{j}^{j}-\epsilon_{j} \mu_{j}^{j} \omega,
\end{gathered}
$$

onde $p_{i j}: \prod_{k \neq i, j} S_{k} \rightarrow \mathcal{Q}, i \in \mathcal{I}, j \in \mathcal{J}, i \neq j, \lambda_{l}, \mu_{l}: \prod_{k \neq l} S_{k} \rightarrow \mathcal{C}, l \in \mathcal{I} \cup \mathcal{J}$, e $\lambda_{l}=0=\mu_{l}$ se $l \notin \mathcal{I} \cap \mathcal{J}$, temos novamente que as I.F.'s são satisfeitas.

Desse modo, definimos (6.34) e (6.35) como sendo as soluções padrão de (6.32) e (6.33).

Vamos agora generalizar o conceito de subconjuntos $d$-livres.

Definição 6.2.1. Seja $d$ um inteiro positivo. Uma tripla $(\hat{S}, \Delta, \hat{\mathcal{U}})$ é chamada $d$-superlivre se as seguintes condições são satisfeitas.

(a) Para todo $m$ inteiro positivo e $\mathcal{I}, \mathcal{J} \subseteq\{1,2, \ldots, m\}$, com $\max \{|\mathcal{I}|,|\mathcal{J}|\} \leq d$, temos que (6.32) implica (6.34) e (6.35).

(b) Para todo $m$ inteiro positivo e $\mathcal{I}, \mathcal{J} \subseteq\{1,2, \ldots, m\}$, com $\max \{|\mathcal{I}|,|\mathcal{J}|\} \leq d-1$, temos que (6.33) implica (6.34) e (6.35).

Se cada $S_{i}=\mathcal{U}_{i}$ e $\delta_{i}=\operatorname{id}_{\mathcal{U}_{i}}$, o subconjunto $\hat{\mathcal{U}}$ é dito ser $d$-superlivre se a tripla $(\hat{S}, \Delta, \hat{\mathcal{U}})$ o for. Se $\mathcal{R}=\mathcal{R}_{0} \oplus \mathcal{R}_{1}$ é um $\Phi$-submódulo graduado de $\mathcal{Q}$, onde $\mathcal{Q}$ é uma álgebra sobre um anel comutativo $\Phi$ e $\frac{1}{2} \in \Phi$, tal que para todo $1 \leq i \leq m, \mathcal{U}_{i}=\mathcal{R}_{0}$ ou $\mathcal{U}_{i}=\mathcal{R}_{1}$, então, $\mathcal{R}$ é dito $d$-superlivre se $\hat{\mathcal{U}}$ é $d$-superlivre.

Observemos que o conceito de subconjuntos $d$-superlivres coincide com o de subconjuntos $d$ livres no caso em que temos uma superálgebra trivial. Além disso, se $\mathcal{I}=\emptyset$ ou $\mathcal{J}=\emptyset$, novamente consideramos que as somas indexadas por estes conjuntos são nulas. 
A seguir, apresentamos as generalizações dos conceitos de funções de núcleo e de funções quasipolinomiais. Sejam $\left\{x_{1}, x_{2}, \ldots, x_{m}\right\}$ um conjunto finito e $k \in \mathbb{N}$, com $k \leq m$. Denotamos por $\mathcal{M}_{m}^{k}$, o conjunto de todos os monômios multilineares em $\left\{x_{1}, x_{2}, \ldots, x_{m}\right\}$ de grau $k$, e fixamos $\mathcal{M}_{m}=\bigcup_{k=0}^{m} \mathcal{M}_{m}^{k}$, onde $\mathcal{M}_{m}^{0}=\{1\}$. Seja $M=X_{i_{1}} X_{i_{2}} \ldots X_{i_{u}} \in \mathcal{M}_{m}$ (aqui denotamos $\operatorname{dom}(M)=$ $\left\{i_{1}, i_{2}, \ldots, i_{u}\right\}$ e $\left.\operatorname{grau}(M)=u\right)$, onde $u \leq(m-k)$. Em seguida, adotamos a notação $\mathcal{M}_{m}^{k}(M)$ para o conjunto de todos os monômios multilineares em $\left\{x_{1}, x_{2}, \ldots, x_{m}\right\} \backslash\left\{x_{i_{1}}, x_{i_{2}}, \ldots, x_{i_{u}}\right\}$ de grau $k$ e fixamos $\mathcal{M}_{m}(M)=\bigcup_{k=0}^{m-u} \mathcal{M}_{m}^{k}$.

Para cada $1 \leq t \leq m$, seja $S_{t}$ um conjunto não vazio e $\delta_{t}: S_{t} \rightarrow \mathcal{U}_{t}$ uma aplicação sobrejetora. Definimos

$$
M\left(\bar{s}_{m}\right)=\delta_{i_{1}}\left(s_{i_{1}}\right) \delta_{i_{2}}\left(s_{i_{2}}\right) \ldots \delta_{i_{u}}\left(s_{i_{u}}\right) \text { e } \epsilon_{M}=\epsilon_{i_{1}} \epsilon_{i_{2}} \ldots \epsilon_{i_{u}}
$$

onde $s_{i_{t}} \in S_{i_{t}}$. Seja $\hat{S}=\prod_{t=1}^{m} S_{i}$ e $\hat{S}^{M}=\prod_{t=1}^{m-u} S_{j_{t}}$, onde

$$
\left\{j_{1}, j_{2}, \ldots, j_{m-u}\right\}=\{1,2, \ldots, m\} \backslash\left\{i_{1}, i_{2}, \ldots, i_{u}\right\} .
$$

Dada $F: \hat{S}^{M} \rightarrow \mathcal{Q}$, definimos

$$
F^{M}\left(\bar{s}_{m}\right)=F\left(s_{j_{1}}, s_{j_{2}}, \ldots, s_{j_{m-u}}\right)
$$

para todo $\bar{s}_{m} \in \hat{S}$. Seja $M \in \mathcal{M}_{m}^{k}$ e $\lambda_{M}: \hat{S}^{M} \rightarrow \mathcal{C}+\mathcal{C} \omega$. Uma aplicação $\hat{S} \rightarrow \mathcal{Q}$ definida pela regra $\bar{s}_{m} \mapsto \lambda_{M}^{M}\left(\bar{s}_{m}\right) M\left(\bar{s}_{m}\right)$, para todo $\bar{s}_{m} \in \hat{S}$, é chamada de superquasi-monomial e é denotada por $\lambda_{M} M$. Uma soma, $\sum_{M \in \mathcal{M}_{m}} \lambda_{M} M$, de diferentes superquasi-monomiais, é dita uma superquasipolinomial de grau menor ou igual a $m, \lambda_{1}$ é o coeficiente central. Em relação às superquasipolinomiais, analogamente à seção anterior, temos o seguinte resultado.

Lema 6.2.1. Sejam $\lambda_{L}: \hat{S}^{L} \rightarrow \mathcal{C}+\mathcal{C} \omega, L \in \mathcal{M}_{m}$, aplicações tais que

$$
\sum_{L \in \mathcal{M}_{m}} \lambda_{L}^{L} L=0
$$

Além disso, suponha que $\lambda_{1}=0$ e $\hat{\mathcal{U}}$ é $m$-superlivre, ou $\hat{\mathcal{U}}$ é $(m+1)$-superlivre. Então, cada $\lambda_{L}$ é nulo.

Demonstração. A demonstração é análoga a do Lema 6.1.3.

Nesse contexto, as funções de núcleo possuem um sentido similar ao da seção anterior: são funções da forma $\sum_{M, N \in \mathcal{M}_{m}} M B_{M, N}^{M N} N$, onde cada par $M, N \in \mathcal{M}_{m}$ de monômios dessa soma, é tal que $\operatorname{dom}(M) \cap \operatorname{dom}(N)=\emptyset$ e $\operatorname{grau}(M N)=m-n$, e $B_{M, N}: \hat{S}^{M N} \rightarrow \mathcal{Q}$ são chamadas de funções de meio. Analogamente, pode-se definir os conceitos de função de meio mais à esquerda e função de meio mais à direita.

As generalizações dos resultados finais da seção anterior podem ser sintetizadas no seguinte teorema, cuja demonstração pode ser encontrada em [32]. 
Teorema 6.2.1. (Yu Wang, [32]) Sejam $m, n \in \mathbb{Z}$, com $0 \leq n<m$. Para cada $i$ tal que $3 \leq$ $i \leq m$, suponha que ou $S_{i}=S_{1}$ e $\delta_{i}=\delta_{1}$, ou $S_{i}=S_{2}$ e $\delta_{i}=\delta_{2}$. Para $M, N \in \mathcal{M}_{m}$, com $\operatorname{dom}(M) \cap \operatorname{dom}(N)=\emptyset$ e $\operatorname{grau}(M N)=m-n$, sejam $a_{M, N} \in \mathcal{C}_{0}$ e $B_{M, N}: \hat{S}^{M N} \longrightarrow \mathcal{Q}$ uma aplicação, e para $L \in \mathcal{M}_{m}$ seja $\lambda_{L}: \hat{S}^{L} \longrightarrow \mathcal{C}+\mathcal{C} \omega$ uma aplicação. Suponha que essas aplicações são tais que

$$
\sum_{M, N} a_{M, N} M B_{M, N}^{M N} N+\sum_{L} \lambda_{L}^{L} L=0
$$

Defina $u=\min \left\{\operatorname{grau}(M) \mid a_{M, N} B_{M, N} \neq 0\right.$ para algum $\left.N\right\}$ e suponha que, para todo $M, N$ tal que $a_{M, N} B_{M, N} \neq 0$, existem $P=P(M, N), Q=Q(M, N) \in \mathcal{M}_{m}, \operatorname{com} \operatorname{dom}(P) \cap \operatorname{dom}(Q)=\emptyset$ e $\operatorname{grau}(P Q)=m-n$, tais que $\operatorname{grau}(P)=u, B_{M, N}=B_{P, Q}$ e $a_{P, Q} \in \mathcal{C}_{0}^{*}$. Além disso, assuma que ou $\hat{\mathcal{U}}$ é $m$-livre e $\lambda_{1}=0$, ou $\hat{\mathcal{U}}$ é $(m+1)$-livre. Então $\lambda_{L}=0$, para todo $L \in \mathcal{M}_{m}, \operatorname{com} \operatorname{grau}(L)<m-n$, e se $a_{M, N} B_{M, N} \neq 0$, existem aplicações $\mu_{M, K, N}: \hat{S}^{M K N} \longrightarrow \mathcal{C}+\mathcal{C} \omega, K \in \mathcal{M}_{m}(M N)$, tais que

$$
B_{M, N}^{M N}=\sum_{K \in \mathcal{M}_{m}(M N)} \mu_{M, K, N}^{M K N} K
$$

Além disso, se $B_{M, N}: \hat{S}^{M N} \longrightarrow \mathcal{Q}_{i}, i=0,1$, então, $\mu_{M, K, N}: \hat{S}^{M K N} \longrightarrow \mathcal{C}_{t}+\mathcal{C}_{t} \omega$, onde $t=$ $\frac{\left|1-\epsilon_{K}-2 i\right|}{2}$.

\subsection{Superhomomorfismos de Jordan em superálgebras}

Como uma aplicação da teoria de identidades funcionais em superálgebras, apresentamos finalmente um resultado tratando dos superhomomorfismos de Jordan.

Vamos recordar algumas notações e definições já fixadas. Seja $\mathcal{A}=\mathcal{A}_{0} \oplus \mathcal{A}_{1}$ uma superálgebra. Para $a, b \in \mathcal{A},[a, b]$ (respectivamente, $a \circ b$ ) denota o comutador (respectivamente, o produto de Jordan) e $[a, b]_{s}$ (respectivamente, $a \circ_{s} b$ ) denota o supercomutador (respectivamente, o superproduto de Jordan). Sejam $\mathcal{B}=\mathcal{B}_{0} \oplus \mathcal{B}_{1}$ uma superálgebra e $\mathcal{I}=\mathcal{I}_{0} \oplus \mathcal{I}_{1}$ uma supersubálgebra de Jordan graduada de $\mathcal{A}$, isto é, $\mathcal{I}$ é um $\Phi$-submódulo graduado de $\mathcal{A}$, tal que para $x, y \in \mathcal{I}$, temos $x \circ_{s} y \in \mathcal{I}$. Uma aplicação graduada $\Phi$-linear $\alpha: \mathcal{I} \rightarrow \mathcal{B}$ é um superhomomorfismo de Jordan se

$$
\alpha\left(x \circ_{s} y\right)=\alpha(x) \circ_{s} \alpha(y)
$$

para todos $x, y \in \mathcal{I}$. Efetuando alguns cálculos, pode-se verificar a validade da seguinte identidade

$$
\left[[s, t]_{s}, u\right]_{s}=s \circ_{s}\left(t \circ_{s} u\right)-(-1)^{|t||s|} t \circ_{s}\left(s \circ_{s} u\right),
$$

para todos $s, t, u \in \mathcal{I}_{0} \cup \mathcal{I}_{1}$, o que implica que, se $\alpha$ é um superhomomorfismo de Jordan, então,

$$
\begin{aligned}
\alpha\left(\left[[s, t]_{s}, u\right]_{s}\right) & =\alpha\left(s \circ_{s}\left(t \circ_{s} u\right)-(-1)^{|t||s|} t \circ_{s}\left(s \circ_{s} u\right)\right) \\
& =\alpha(s) \circ_{s}\left(\alpha(t) \circ_{s} \alpha(u)\right)-(-1)^{|\alpha(t)||\alpha(s)|} \alpha(t) \circ_{s}\left(\alpha(s) \circ_{s} \alpha(u)\right) \\
& =\left[[\alpha(s), \alpha(t)]_{s}, \alpha(u)\right]_{s},
\end{aligned}
$$

isto é,

$$
\alpha\left(\left[[s, t]_{s}, u\right]_{s}\right)=\left[[\alpha(s), \alpha(t)]_{s}, \alpha(u)\right]_{s} .
$$


Seja $\mathcal{Q}=\mathcal{Q}_{0} \oplus \mathcal{Q}_{1}$ uma superálgebra com identidade e centro $\mathcal{C}=\mathcal{C}_{0} \oplus \mathcal{C}_{1}$. Assim como definimos na seção anterior, $\omega$ denotará o elemento graduante de $\mathcal{Q}$. Se $a=b+c \omega$, onde $b, c \in \mathcal{C}$, fixamos $\bar{a}=b-c \omega$. Observe que, pelas propriedades de $\omega$, se $x_{0} \in \mathcal{Q}_{0}$ e $x_{1} \in \mathcal{Q}_{1}$, então, $a x_{0}=x_{0} a$ e $a x_{1}=x_{1} \bar{a}$. Além disso, claramente $\overline{\bar{a}}=a$.

Já estamos em condições de provar o seguinte teorema. Observemos que a seguir, a expressão "soma direta" tem o mesmo significado da seção anterior, isto é, existe um idempotente central $\epsilon \in \mathcal{Q}$, tal que $x \mapsto \epsilon x$ é um superhomomorfismo, e $x \mapsto(1-\epsilon) x$ é um superantihomomorfismo.

Teorema 6.3.1. (Yao Wang e Yu Wang, [33]) Sejam $\mathcal{A}=\mathcal{A}_{0} \oplus \mathcal{A}_{1}$ uma superálgebra e $\mathcal{Q}=\mathcal{Q}_{0} \oplus \mathcal{Q}_{1}$ uma superálgebra com identidade e centro $\mathcal{C}=\mathcal{C}_{0} \oplus \mathcal{C}_{1}$. Se $\alpha: \mathcal{A} \rightarrow \mathcal{Q}$ é um superhomomorfismo de Jordan tal que $\alpha(\mathcal{A})$ é um subconjunto 4-superlivre de $\mathcal{Q}$, então, $\alpha$ é soma direta de um superhomomorfismo com um superantihomomorfismo.

Demonstração. Por hipótese, de (6.36), obtemos

$$
\begin{aligned}
{\left[[\alpha(x), \alpha(y)]_{s}, \alpha\left([z, w]_{s}\right)\right]_{s} } & =\alpha\left(\left[[x, y]_{s},[z, w]_{s}\right]_{s}\right) \\
& = \pm \alpha\left(\left[[z, w]_{s},[x, y]_{s}\right]_{s}\right) \\
& = \pm\left[[\alpha(z), \alpha(w)]_{s}, \alpha\left([x, y]_{s}\right)\right]_{s} \\
& =\left[\alpha\left([x, y]_{s}\right),[\alpha(z), \alpha(w)]_{s}\right]_{s}
\end{aligned}
$$

para todos $x, y, z, w \in \mathcal{A}_{0} \cup \mathcal{A}_{1}$, o que reescrevemos do seguinte modo

$$
\left[[\alpha(x), \alpha(y)]_{s}, B(z, w)\right]_{s}=\left[B(x, y),[\alpha(z), \alpha(w)]_{s}\right]_{s}
$$

para todos $x, y, z, w \in \mathcal{A}_{0} \cup \mathcal{A}_{1}$, onde $B(x, y)=\alpha\left([x, y]_{s}\right)$. Assim, como $\alpha(\mathcal{A})$ é um subconjunto 4-superlivre de $\mathcal{Q}$, segue do Teorema 6.2.1, que $B$ é uma superquasi-polinomial, isto é,

$$
\begin{aligned}
B\left(x_{0}, y_{0}\right)= & \lambda_{0}^{1} \alpha\left(x_{0}\right) \alpha\left(y_{0}\right)+\lambda_{1}^{1} \alpha\left(y_{0}\right) \alpha\left(x_{0}\right)+\mu_{1}^{1}\left(x_{0}\right) \alpha\left(y_{0}\right) \\
& +\mu_{2}^{1}\left(y_{0}\right) \alpha\left(x_{0}\right)+\gamma^{1}\left(x_{0}, y_{0}\right), \\
B\left(x_{0}, y_{1}\right)= & \lambda_{0}^{2} \alpha\left(x_{0}\right) \alpha\left(y_{1}\right)+\lambda_{1}^{2} \alpha\left(y_{1}\right) \alpha\left(x_{0}\right)+\mu_{1}^{2}\left(x_{0}\right) \alpha\left(y_{1}\right) \\
& +\mu_{2}^{2}\left(y_{1}\right) \alpha\left(x_{0}\right)+\gamma^{2}\left(x_{0}, y_{1}\right), \\
B\left(x_{1}, y_{1}\right)= & \lambda_{0}^{3} \alpha\left(x_{1}\right) \alpha\left(y_{1}\right)+\lambda_{1}^{3} \alpha\left(y_{1}\right) \alpha\left(x_{1}\right)+\mu_{1}^{3}\left(x_{1}\right) \alpha\left(y_{1}\right) \\
& +\mu_{2}^{3}\left(y_{1}\right) \alpha\left(x_{1}\right)+\gamma^{3}\left(x_{1}, y_{1}\right),
\end{aligned}
$$

para todos $x_{0}, y_{0} \in \mathcal{A}_{0}, x_{1}, y_{1} \in \mathcal{A}_{1}$, onde $\lambda_{0}^{i}, \lambda_{1}^{i} \in \mathcal{C}_{0}+\mathcal{C}_{0} \omega, i=1,2,3, \mu_{1}^{1}, \mu_{1}^{2}, \mu_{2}^{1}: \mathcal{A}_{0} \rightarrow \mathcal{C}_{0}+\mathcal{C}_{0} \omega$, $\mu_{1}^{3}, \mu_{2}^{2}, \mu_{2}^{3}: \mathcal{A}_{1} \rightarrow \mathcal{C}_{1}+\mathcal{C}_{1} \omega, \gamma^{1}: \mathcal{A}_{0}^{2} \rightarrow \mathcal{C}_{0}+\mathcal{C}_{0} \omega, \gamma^{2}: \mathcal{A}_{0} \times \mathcal{A}_{1} \rightarrow \mathcal{C}_{1}+\mathcal{C}_{1} \omega$, e $\gamma^{3}: \mathcal{A}_{1}^{2} \rightarrow \mathcal{C}_{0}+\mathcal{C}_{0} \omega . \mathrm{Em}$ particular, de (6.37), obtemos

$$
\left[\left[\alpha\left(x_{0}\right), \alpha\left(y_{0}\right)\right]_{s}, B\left(z_{0}, w_{0}\right)\right]_{s}=\left[B\left(x_{0}, y_{0}\right),\left[\alpha\left(z_{0}\right), \alpha\left(w_{0}\right)\right]_{s}\right]_{s},
$$

para todos $x_{0}, y_{0}, z_{0}, w_{0} \in \mathcal{A}_{0}$. Agora, substituindo (6.38) em (6.41), da última igualdade encontrada, temos que

- o coeficiente de $\alpha\left(x_{0}\right) \alpha\left(y_{0}\right) \alpha\left(w_{0}\right) \alpha\left(z_{0}\right)$ é $\lambda_{0}^{1}+\lambda_{1}^{1}$;

- o coeficiente de $\alpha\left(x_{0}\right) \alpha\left(y_{0}\right) \alpha\left(w_{0}\right)$ é $\mu_{1}^{1}\left(z_{0}\right)$; 
- o coeficiente de $\alpha\left(x_{0}\right) \alpha\left(y_{0}\right) \alpha\left(z_{0}\right)$ é $\mu_{2}^{1}\left(w_{0}\right)$.

Desse modo, como $\alpha(\mathcal{A})$ é 4-superlivre e o coeficiente central da superquasi-polinomial, dada pela substituição anterior, é igual a 0 , resulta $\lambda_{0}^{1}+\lambda_{1}^{1}=0$ e que $\mu_{1}^{1}=\mu_{2}^{1}=0$, pelo Lema 6.2.1. Assim,

$$
\begin{aligned}
\alpha\left(\left[x_{0}, y_{0}\right]_{s}\right) & =B\left(x_{0}, y_{0}\right) \\
& =\lambda_{0}^{1}\left[\alpha\left(x_{0}\right), \alpha\left(y_{0}\right)\right]+\gamma^{1}\left(x_{0}, y_{0}\right), \text { para todos } x_{0}, y_{0} \in \mathcal{A}_{0} .
\end{aligned}
$$

Analogamente, por (6.37), temos

$$
\left[\left[\alpha\left(x_{0}\right), \alpha\left(y_{1}\right)\right]_{s}, B\left(z_{0}, w_{1}\right)\right]_{s}=\left[B\left(x_{0}, y_{1}\right),\left[\alpha\left(z_{0}\right), \alpha\left(w_{1}\right)\right]_{s}\right]_{s}
$$

para todos $x_{0}, z_{0} \in \mathcal{A}_{0}, y_{1}, w_{1} \in \mathcal{A}_{1}$. Portanto, substituindo (6.39) em (6.42), da igualdade imediatamente obtida, segue que

- o coeficiente de $\alpha\left(x_{0}\right) \alpha\left(y_{1}\right) \alpha\left(w_{1}\right) \alpha\left(z_{0}\right)$ é $\overline{\lambda_{1}^{2}}+\lambda_{0}^{2}$;

- o coeficiente de $\alpha\left(x_{0}\right) \alpha\left(y_{1}\right) \alpha\left(z_{0}\right) \alpha\left(w_{1}\right)$ é $\overline{\lambda_{0}^{2}}-\lambda_{0}^{2}$;

- o coeficiente de $\alpha\left(x_{0}\right) \alpha\left(y_{1}\right) \alpha\left(w_{1}\right)$ é $\overline{\mu_{1}^{2}\left(z_{0}\right)}$;

- o coeficiente de $\alpha\left(x_{0}\right) \alpha\left(y_{1}\right) \alpha\left(z_{0}\right)$ é $\overline{\mu_{2}^{2}\left(w_{1}\right)}$.

Assim, pelo Lema 6.2.1, temos $\overline{\lambda_{1}^{2}}+\lambda_{0}^{2}=0, \overline{\lambda_{0}^{2}}-\lambda_{0}^{2}$ e $\overline{\mu_{1}^{2}}=\overline{\mu_{2}^{2}}=0$, o que implica $\lambda_{0}^{2}=-\lambda_{1}^{2} \in \mathcal{C}_{0}$, pois é igual ao seu conjugado, e $\mu_{1}^{2}=\mu_{2}^{2}=0$. Logo,

$$
\begin{aligned}
\alpha\left(\left[x_{0}, y_{1}\right]_{s}\right) & =B\left(x_{0}, y_{1}\right) \\
& =\lambda_{0}^{2}\left[\alpha\left(x_{0}\right), \alpha\left(y_{1}\right)\right]+\gamma^{2}\left(x_{0}, y_{1}\right), \quad \text { para todos } x_{0} \in \mathcal{A}_{0} \text { e } y_{1} \in \mathcal{A}_{1} .
\end{aligned}
$$

Agora, por (6.37), temos

$$
\left[\left[\alpha\left(x_{0}\right), \alpha\left(y_{0}\right)\right]_{s}, B\left(z_{0}, w_{1}\right)\right]_{s}=\left[B\left(x_{0}, y_{0}\right),\left[\alpha\left(z_{0}\right), \alpha\left(w_{1}\right)\right]_{s}\right]_{s}
$$

para todos $x_{0}, y_{0}, z_{0} \in \mathcal{A}_{0}$ e $w_{1} \in \mathcal{A}_{1}$. Substituindo (6.38) e (6.39) em (6.43), da identidade obtida podemos concluir que o coeficiente de $\alpha\left(x_{0}\right) \alpha\left(y_{0}\right) \alpha\left(z_{0}\right) \alpha\left(w_{1}\right)$ é $\lambda_{0}^{2}-\lambda_{0}^{1}$. Desse modo, pelo Lema (6.2.1), temos $\lambda_{0}^{2}=\lambda_{0}^{1}$. Além disso, como

$$
\begin{aligned}
B\left(x_{1}, y_{0}\right) & =\alpha\left(\left[x_{1}, y_{0}\right]_{s}\right)=-\alpha\left(\left[y_{0}, x_{1}\right]_{s}\right) \\
& =-B\left(y_{0}, x_{1}\right), \text { para todos } x_{1} \in \mathcal{A}_{1} \text { e } y_{0} \in \mathcal{A}_{0}
\end{aligned}
$$

as seguintes igualdades são válidas:

$$
\begin{aligned}
\alpha\left(\left[x_{1}, y_{0}\right]_{s}\right) & =B\left(x_{1}, y_{0}\right)=-B\left(y_{0}, x_{1}\right) \\
& =-\lambda_{0}^{2}\left[\alpha\left(y_{0}\right), \alpha\left(x_{1}\right)\right]-\gamma^{2}\left(y_{0}, x_{1}\right) \\
& =\lambda_{0}^{1}\left[\alpha\left(x_{1}\right), \alpha\left(y_{0}\right)\right]-\gamma^{2}\left(y_{0}, x_{1}\right), \text { para todos } x_{1} \in \mathcal{A}_{1} \text { e } y_{0} \in \mathcal{A}_{0} .
\end{aligned}
$$

Finalmente, por (6.37), obtemos uma última igualdade:

$$
\left[\left[\alpha\left(x_{1}\right), \alpha\left(y_{1}\right)\right]_{s}, B\left(z_{0}, w_{1}\right)\right]_{s}=\left[B\left(x_{1}, y_{1}\right),\left[\alpha\left(z_{0}\right), \alpha\left(w_{1}\right)\right]_{s}\right]_{s},
$$


para todos $z_{0} \in \mathcal{A}_{0}$ e $x_{1}, y_{1}, z_{1} \in \mathcal{A}_{1}$. Assim, substituindo ambos (6.39) e (6.40) em (6.44), da igualdade obtida segue que

- o coeficiente de $\alpha\left(x_{1}\right) \alpha\left(y_{1}\right) \alpha\left(w_{1}\right) \alpha\left(z_{0}\right)$ é $-\lambda_{0}^{1}+\lambda_{0}^{3}$;

- o coeficiente de $\alpha\left(y_{1}\right) \alpha\left(x_{1}\right) \alpha\left(z_{0}\right) \alpha\left(w_{1}\right)$ é $\lambda_{0}^{1}-\lambda_{1}^{3}$;

- o coeficiente de $\alpha\left(x_{1}\right) \alpha\left(z_{0}\right) \alpha\left(w_{1}\right)$ é $\mu_{2}^{3}\left(y_{1}\right)$;

- o coeficiente de $\alpha\left(y_{1}\right) \alpha\left(z_{0}\right) \alpha\left(w_{1}\right)$ é $\mu_{1}^{3}\left(x_{1}\right)$.

Portanto, pelo Lema 6.2.1, obtemos $\lambda_{0}^{1}=\lambda_{0}^{3}=\lambda_{1}^{3}$ e $\mu_{1}^{3}=\mu_{2}^{3}=0$. Assim,

$$
\begin{aligned}
\alpha\left(\left[x_{1}, y_{1}\right]_{s}\right) & =B\left(x_{1}, y_{1}\right) \\
& =\lambda_{0}^{1}\left[\alpha\left(x_{1}\right), \alpha\left(y_{1}\right)\right]_{s}+\gamma^{3}\left(x_{1}, y_{1}\right), \text { para todos } x_{1}, y_{1} \in \mathcal{A}_{1} .
\end{aligned}
$$

Fixe $\lambda=\lambda_{0}^{1}$. As identidades anteriores podem ser reescritas como

$$
\alpha\left([x, y]_{s}\right)=\lambda[\alpha(x), \alpha(y)]_{s}+\gamma(x, y)
$$

para todos $x, y \in \mathcal{A}_{0} \cup \mathcal{A}_{1}$, onde $\gamma: \mathcal{A}^{2} \rightarrow \mathcal{C}+\mathcal{C} \omega$ é uma aplicação ( $\gamma$ "alterna" entre $\gamma^{1}, \gamma^{2}$ e $\gamma^{3}$ ).

Desse modo, como $\alpha$ é um superhomomorfismo de Jordan, vale $\alpha\left(x \circ_{s} y\right)=\alpha(x) \circ_{s} \alpha(y)$, para $x, y \in \mathcal{A}_{0} \cup \mathcal{A}_{1}$. Reescrevendo esta igualdade, temos

$$
\alpha(x y)=\alpha(x) \alpha(y)+(-1)^{|\alpha(x)||\alpha(y)|} \alpha(y) \alpha(x)-(-1)^{|x||y|} \alpha(y x) .
$$

Logo, por (6.45), segue

$$
\alpha(x y)=\lambda \alpha(x) \alpha(y)-(-1)^{|\alpha(x)||\alpha(y)|} \lambda \alpha(y) \alpha(x)+\gamma(x, y)+(-1)^{|x||y|} \alpha(y x) .
$$

Somando membro a membro essas duas últimas igualdades e usando o fato que $\alpha$ preserva a $\mathbb{Z}_{2 \text {-graduação, chegamos a }}$

$$
\alpha(x y)=\epsilon \alpha(x) \alpha(y)+(-1)^{|x||y|} \epsilon^{\prime} \alpha(y) \alpha(x)+\frac{1}{2} \gamma(x, y),
$$

onde $\epsilon=\frac{1}{2}(1+\lambda)$ e $\epsilon^{\prime}=\frac{1}{2}(1-\lambda)$, e portanto $\epsilon+\epsilon^{\prime}=1$.

Agora, para $x_{0} \in \mathcal{A}_{0}$ e $x, z \in \mathcal{A}_{0} \cup \mathcal{A}_{1}$, vamos calcular, de dois modos distintos, $\alpha\left(x_{0} y z\right)$. Por um lado, temos

$$
\begin{aligned}
\alpha\left(\left(x_{0} y\right) z\right)= & \epsilon \alpha\left(x_{0} y\right) \alpha(z)+(-1)^{|y||z|} \epsilon^{\prime} \alpha(z) \alpha\left(x_{0} y\right)+\frac{1}{2} \gamma\left(x_{0} y, z\right) \\
= & \epsilon^{2} \alpha\left(x_{0}\right) \alpha(y) \alpha(z)+\epsilon \epsilon^{\prime} \alpha(y) \alpha\left(x_{0}\right) \alpha(z)+\frac{1}{2} \epsilon \gamma\left(x_{0}, y\right) \alpha(z) \\
& +(-1)^{|y||z|} \epsilon \epsilon^{\prime} \alpha(z) \alpha\left(x_{0}\right) \alpha(y)+(-1)^{|y||z|} \epsilon^{\prime 2} \alpha(z) \alpha(y) \alpha\left(x_{0}\right) \\
& +(-1)^{|y||z|} \frac{1}{2} \epsilon^{\prime} \alpha(z) \gamma\left(x_{0}, y\right)+\frac{1}{2} \gamma\left(x_{0} y, z\right),
\end{aligned}
$$


onde $\lambda=\lambda_{0}^{1}=\lambda_{0}^{2} \in \mathcal{C}_{0}$, e portanto, $\epsilon$ e $\epsilon^{\prime}$ pertencem a $\mathcal{C}_{0}$. Por outro lado, também temos

$$
\begin{aligned}
\alpha\left(x_{0}(y z)\right)= & \epsilon \alpha\left(x_{0}\right) \alpha(y z)+\epsilon^{\prime} \alpha(y z) \alpha\left(x_{0}\right)+\frac{1}{2} \gamma\left(x_{0}, y z\right) \\
= & \epsilon^{2} \alpha\left(x_{0}\right) \alpha(y) \alpha(z)+(-1)^{|y||z|} \epsilon \epsilon^{\prime} \alpha\left(x_{0}\right) \alpha(z) \alpha(y)+\frac{1}{2} \epsilon \gamma(y, z) \alpha\left(x_{0}\right) \\
& +\epsilon \epsilon^{\prime} \alpha(y) \alpha(z) \alpha\left(x_{0}\right)+(-1)^{|y||z|} \epsilon^{\prime 2} \alpha(z) \alpha(y) \alpha\left(x_{0}\right)+\frac{1}{2} \epsilon^{\prime} \gamma(y, z) \alpha\left(x_{0}\right) \\
& +\frac{1}{2} \gamma\left(x_{0}, y z\right) .
\end{aligned}
$$

Comparando estas duas expressões, obtemos

$$
\begin{array}{r}
\epsilon \epsilon^{\prime}\left[\alpha(y),\left[\alpha\left(x_{0}\right), \alpha(z)\right]\right]_{s}+\frac{1}{2} \epsilon \gamma\left(x_{0}, y\right) \alpha(z)+(-1)^{|y||z|} \frac{1}{2} \epsilon^{\prime} \alpha(z) \gamma\left(x_{0}, y\right)-\frac{1}{2} \gamma(y, z) \alpha\left(x_{0}\right) \\
=-\frac{1}{2}\left(\gamma\left(x_{0} y, z\right)-\gamma\left(x_{0}, y z\right)\right),
\end{array}
$$

ou seja,

$$
\begin{aligned}
\epsilon \epsilon^{\prime}\left[\alpha(y),\left[\alpha\left(x_{0}\right), \alpha(z)\right]\right]_{s}+\frac{1}{2} \epsilon \gamma\left(x_{0}, y\right) \alpha(z) \\
\quad+(-1)^{|y||z|} \frac{1}{2} \epsilon^{\prime} \alpha(z) \gamma\left(x_{0}, y\right)-\frac{1}{2} \gamma(y, z) \alpha\left(x_{0}\right) \in \mathcal{C}+\mathcal{C} \omega .
\end{aligned}
$$

Em particular, tomando $z=z_{0} \in \mathcal{A}_{0}$ em (6.46), segue

$$
\epsilon \epsilon^{\prime}\left[\alpha(y),\left[\alpha\left(x_{0}\right), \alpha\left(z_{0}\right)\right]\right]_{s}+\frac{1}{2} \gamma\left(x_{0}, y\right) \alpha\left(z_{0}\right)-\frac{1}{2} \gamma\left(y, z_{0}\right) \alpha\left(x_{0}\right) \in \mathcal{C}+\mathcal{C} \omega
$$

para todos $x_{0}, z_{0} \in \mathcal{A}_{0}$ e $y \in \mathcal{A}_{0} \cup \mathcal{A}_{1}$, pois $\alpha\left(z_{0}\right) \in \mathcal{Q}_{0}$ e $\gamma\left(x_{0}, y\right) \in \mathcal{C}+\mathcal{C} \omega$. Observe que essa última pertinência, resulta em uma I.F. que iguala uma superquasi-polinomial em 3 variáveis a 0 . Portanto, pelo Lema 6.2.1, seguem $\epsilon \epsilon^{\prime}=0$ e $\gamma\left(y, z_{0}\right)=0$, para todos $y \in \mathcal{A}_{0} \cup \mathcal{A}_{1}$ e $z_{0} \in \mathcal{A}_{0}$.

Analogamente, tomando $z=z_{1} \in \mathcal{A}_{1}$ em (6.46), obtemos

$$
\epsilon \epsilon^{\prime}\left[\alpha(y),\left[\alpha\left(x_{0}\right), \alpha\left(z_{1}\right)\right]\right]_{s}+\frac{1}{2}\left(\epsilon \gamma\left(x_{0}, y\right)+(-1)^{|y|} \epsilon^{\prime} \overline{\gamma\left(x_{0}, y\right)}\right) \alpha\left(z_{1}\right)-\frac{1}{2} \gamma\left(y, z_{0}\right) \alpha\left(x_{0}\right) \in \mathcal{C}+\mathcal{C} \omega,
$$

e novamente aplicando o Lema 6.2.1, concluímos $\gamma\left(y, z_{1}\right)=0$, para todos $y \in \mathcal{A}_{0} \cup \mathcal{A}_{1}$ e $z_{1} \in \mathcal{A}_{1}$. Assim, $\gamma(y, z)=0$, para quaisquer $y, z \in \mathcal{A}_{0} \cup \mathcal{A}_{1}$, isto é, $\gamma$ é identicamente nula.

Além disso, $\epsilon$ e $\epsilon^{\prime}$ são idempotentes contidos no centro de $\mathcal{Q}$. De fato, já observamos anteriormente $\epsilon, \epsilon^{\prime} \in \mathcal{C}_{0}$. Assim, como $\epsilon \epsilon^{\prime}=0$ e $\epsilon+\epsilon^{\prime}=1$, obtemos

$$
\begin{gathered}
\epsilon=\epsilon\left(\epsilon+\epsilon^{\prime}\right)=\epsilon^{2} \mathrm{e} \\
\epsilon^{\prime}=\epsilon^{\prime}\left(\epsilon+\epsilon^{\prime}\right)=\epsilon^{\prime 2} .
\end{gathered}
$$

Agora, temos

$$
\alpha(x y)=\epsilon \alpha(x) \alpha(y)+(-1)^{|x||y|} \epsilon^{\prime} \alpha(y) \alpha(x),
$$

para todos $x, y \in \mathcal{A}_{0} \cup \mathcal{A}_{1}$. Definindo $\alpha_{1}: \mathcal{A} \rightarrow \mathcal{Q}$ por $\alpha_{1}(x)=\epsilon \alpha(x)$, para todo $x \in \mathcal{A}$, e $\alpha_{2}: \mathcal{A} \rightarrow \mathcal{Q}$ por $\alpha_{2}(x)=\epsilon^{\prime} \alpha(x)$, para qualquer $x \in \mathcal{A}$, segue que $\alpha_{1}$ e $\alpha_{2}$ são aplicações $\Phi$-lineares que preservam a $\mathbb{Z}_{2 \text {-graduação, satisfazendo }}$

$$
\begin{aligned}
\alpha_{1}(x y) & =\epsilon \alpha(x y)=\epsilon\left(\epsilon \alpha(x) \alpha(y)+(-1)^{|x||y|} \epsilon^{\prime} \alpha(y) \alpha(x)\right) \\
& =(\epsilon \alpha(x))(\epsilon \alpha(y))=\alpha_{1}(x) \alpha_{1}(y) \mathrm{e}
\end{aligned}
$$




$$
\begin{aligned}
\alpha_{2}(x y) & =\epsilon^{\prime} \alpha(x y)=\epsilon^{\prime}\left(\epsilon \alpha(x) \alpha(y)+(-1)^{|x||y|} \epsilon^{\prime} \alpha(y) \alpha(x)\right) \\
& =(-1)^{|x||y|}\left(\epsilon^{\prime} \alpha(y)\right)\left(\epsilon^{\prime} \alpha(x)\right)=(-1)^{|x||y|} \alpha_{2}(y) \alpha_{2}(x),
\end{aligned}
$$

ou seja, $\alpha_{1}$ é um superhomomorfismo e $\alpha_{2}$ é um superantihomomorfismo. Desse modo, existe um idempotente central $\epsilon$, tal que $\alpha(x)=\epsilon \alpha(x)+(1-\epsilon) \alpha(x)$, para todo $x \in \mathcal{A}, \epsilon \alpha$ é um superhomomorfismo e $\epsilon^{\prime} \alpha$ é um superantihomomorfismo.

Dando sequência, analogamente ao que foi feito na primeira seção deste capítulo, desejamos demonstrar uma generalização do Corolário 6.1.2. No entanto, nos restringiremos às superderivações de Jordan de grau 0. Inicialmente, é necessário que façamos a seguinte generalização do Lema 6.1.2. Vale ressaltar que os próximos resultados são inéditos.

Lema 6.3.1. Se $\mathcal{A}=\mathcal{A}_{0} \oplus \mathcal{A}_{1}$ é um $\Phi$-submódulo graduado $d$-superlivre da superálgebra $\mathcal{Q}=$ $\mathcal{Q}_{0} \oplus \mathcal{Q}_{1}$, com identidade e centro $\mathcal{C}=\mathcal{C}_{0} \oplus \mathcal{C}_{1}$, e $\delta: \mathcal{A} \rightarrow \mathcal{Q}$ é uma aplicação $\Phi$-linear, que preserva a $\mathbb{Z}_{2}$-graduação, então, $\breve{\mathcal{A}}=\{(x, \delta(x)) \mid x \in \mathcal{A}\}$ é um $\Phi$-submódulo graduado $d$-superlivre de $\breve{\mathcal{Q}}$, onde $\breve{\mathcal{Q}}=\mathcal{Q} \times \mathcal{Q}$ é a superálgebra com as operações

- $(x, y)+(z, w)=(x+z, y+w)$,

- $k(x, y)=(k x, k y)$

- $(x, y)(z, w)=(x z, x w+y z)$,

para todos $x, y, z, w \in \mathcal{Q}$, para todo $k \in \Phi$ ( $\mathcal{Q}$ é uma álgebra sobre um anel comutativo $\Phi$, tal que $\left.\frac{1}{2} \in \Phi\right)$, e com a $\mathbb{Z}_{2}$-graduação $\breve{\mathcal{Q}}_{0}=\mathcal{Q}_{0} \times \mathcal{Q}_{0}, \breve{\mathcal{Q}}_{1}=\mathcal{Q}_{1} \times \mathcal{Q}_{1}$.

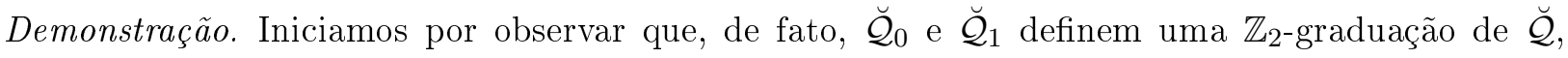
pois estes são $\Phi$-submódulos de $\breve{\mathcal{Q}}$ (já que $\mathcal{Q}_{0}$ e $\mathcal{Q}_{1}$ são $\Phi$-submódulos de $\mathcal{Q}$ ), tais que, para cada $(x, y) \in \breve{\mathcal{Q}}$

$$
\begin{gathered}
(x, y)=\left(x_{0}+x_{1}, y_{0}+y_{1}\right)=\left(x_{0}, y_{0}\right)+\left(x_{1}, y_{1}\right) \in \breve{\mathcal{Q}}_{0}+\breve{\mathcal{Q}}_{1}, \\
\breve{\mathcal{Q}}_{0} \cap \breve{\mathcal{Q}}_{1}=\left(\mathcal{Q}_{0} \times \mathcal{Q}_{0}\right) \cap\left(\mathcal{Q}_{1} \times \mathcal{Q}_{1}\right)=\left(\mathcal{Q}_{0} \cap \mathcal{Q}_{1}\right) \times\left(\mathcal{Q}_{0} \cap \mathcal{Q}_{1}\right)=0 \times 0=0 \mathrm{e}
\end{gathered}
$$

$\breve{\mathcal{Q}}_{i} \breve{\mathcal{Q}}_{j} \subseteq \breve{\mathcal{Q}}_{i+j(\bmod 2)}$. Também temos que $\breve{\mathcal{A}}$ é um $\Phi$-submódulo, pois $\delta$ é uma aplicação $\Phi$-linear, e se $(x, \delta(x)) \in \breve{\mathcal{A}}$, como $\delta$ preserva a $\mathbb{Z}_{2}$-graduação, segue que valem

$$
\begin{aligned}
\breve{\sigma}(x, \delta(x)) & =\breve{\sigma}\left(\left(x_{0}, \delta\left(x_{0}\right)\right)+\left(x_{1}, \delta\left(x_{1}\right)\right)\right) \\
& =\left(x_{0}, \delta\left(x_{0}\right)\right)-\left(x_{1}, \delta\left(x_{1}\right)\right) \\
& =(\sigma(x), \delta(\sigma(x))) \in \breve{\mathcal{A}},
\end{aligned}
$$

onde $\breve{\sigma}$ é o automorfismo definido pela $\mathbb{Z}_{2}$-graduação de $\breve{\mathcal{Q}}$ e $\sigma$ é o automorfismo definido pela $\mathbb{Z}_{2}$-graduação de $\mathcal{Q}$, pois $\sigma(\mathcal{A})=\mathcal{A}$, uma vez que $\mathcal{A}$ é graduado. Logo, $\breve{\sigma}(\breve{\mathcal{A}}) \subseteq \breve{\mathcal{A}}$, o que implica $\breve{\sigma}^{2}(\breve{\mathcal{A}}) \subseteq \breve{\sigma}(\breve{\mathcal{A}})$. Portanto, $\breve{\mathcal{A}} \subseteq \breve{\sigma}(\breve{\mathcal{A}})$, pois $\breve{\sigma}^{2}=\mathrm{id}_{\breve{\mathcal{Q}}}$. Logo, $\breve{\mathcal{A}}$ é graduado.

Agora, vamos provar somente a condição (a) da Definição 6.2.1, pois a demonstração de (b) é análoga. Sejam $m$ um inteiro positivo e $\mathcal{I}, \mathcal{J} \subseteq\{1,2, \ldots, m\}$ subconjuntos, tais que $\max \{|\mathcal{I}|,|\mathcal{J}|\} \leq d$. Para cada $i \in\{1,2, \ldots, m\}$, considere conjuntos $U_{i}$, tais que $U_{i}=\breve{\mathcal{A}}_{0}=\breve{\mathcal{A}} \cap \breve{\mathcal{Q}}_{0}=\left\{\left(x_{0}, \delta\left(x_{0}\right)\right) ; x_{0} \in\right.$ 
$\left.\mathcal{A}_{0}\right\}$ ou $U_{i}=\breve{\mathcal{A}}_{1}=\breve{\mathcal{A}} \cap \breve{\mathcal{Q}}_{1}=\left\{\left(x_{1}, \delta\left(x_{1}\right)\right) ; x_{1} \in \mathcal{A}_{1}\right\}$. Sejam $\breve{E}_{i}: \hat{U}^{i} \rightarrow \breve{\mathcal{Q}}, \breve{F}_{j}: \hat{U}^{j} \rightarrow \breve{\mathcal{Q}}$ aplicações, e consideremos a identidade funcional

$$
\sum_{i \in \mathcal{I}} \breve{E}_{i}\left(\bar{u}_{m}^{i}\right) u_{i}+\sum_{j \in \mathcal{J}} u_{j} \breve{F}_{j}\left(\bar{u}_{m}^{j}\right)=0
$$

Desejamos provar que esta I.F. possui apenas a solução padrão. Para cada $i, u_{i}=\left(x_{i}, \delta\left(x_{i}\right)\right)$, onde $x_{i} \in \mathcal{A}_{0}$ ou $x_{i} \in \mathcal{A}_{1}$. Desse modo, $u_{i}$ é univocamente determinado por $x_{i}$, o que nos permite escrever

$$
\begin{aligned}
& \breve{E}_{i}\left(\bar{u}_{m}^{i}\right)=\left(E_{i}\left(\bar{x}_{m}^{i}\right), G_{i}\left(\bar{x}_{m}^{i}\right)\right), \\
& \breve{F}_{j}\left(\bar{u}_{m}^{j}\right)=\left(F_{j}\left(\bar{x}_{m}^{j}\right), H_{j}\left(\bar{x}_{m}^{j}\right)\right),
\end{aligned}
$$

onde $E_{i}, G_{i}: \hat{V}^{i} \rightarrow \mathcal{Q}$ e $F_{j}, H_{j}: \hat{V}^{j} \rightarrow \mathcal{Q}$ são aplicações, $\hat{V}=\prod_{i=1}^{m} V_{i}, V_{i}=\mathcal{A}_{0}$ ou $V_{i}=\mathcal{A}_{1}$. Substituindo na I.F. (6.47), temos

$$
\sum_{i \in \mathcal{I}}\left(E_{i}\left(\bar{x}_{m}^{i}\right), G_{i}\left(\bar{x}_{m}^{i}\right)\right)\left(x_{i}, \delta\left(x_{i}\right)\right)+\sum_{j \in \mathcal{J}}\left(x_{j}, \delta\left(x_{j}\right)\right)\left(F_{j}\left(\bar{x}_{m}^{j}\right), H_{j}\left(\bar{x}_{m}^{j}\right)\right)=0
$$

isto é,

$$
\sum_{i \in \mathcal{I}}\left(E_{i}\left(\bar{x}_{m}^{i}\right) x_{i}, E_{i}\left(\bar{x}_{m}^{i}\right) \delta\left(x_{i}\right)+G_{i}\left(\bar{x}_{m}^{i}\right) x_{i}\right)+\sum_{j \in \mathcal{J}}\left(x_{j} F_{j}\left(\bar{x}_{m}^{j}\right), x_{j} H_{j}\left(\bar{x}_{m}^{j}\right)+\delta\left(x_{j}\right) F_{j}\left(\bar{x}_{m}^{j}\right)\right)=0
$$

de onde retiramos duas identidades funcionais:

$$
\begin{gathered}
\sum_{i \in \mathcal{I}} E_{i}\left(\bar{x}_{m}^{i}\right) x_{i}+\sum_{j \in \mathcal{J}} x_{j} F_{j}\left(\bar{x}_{m}^{j}\right)=0, \\
\sum_{i \in \mathcal{I}}\left\{E_{i}\left(\bar{x}_{m}^{i}\right) \delta\left(x_{i}\right)+G_{i}\left(\bar{x}_{m}^{i}\right) x_{i}\right\}+\sum_{j \in \mathcal{J}}\left\{\delta\left(x_{j}\right) F_{j}\left(\bar{x}_{m}^{j}\right)+x_{j} H_{j}\left(\bar{x}_{m}^{j}\right)\right\}=0 .
\end{gathered}
$$

A seguir, dividiremos a demonstração em dois casos. Primeiramente, assumimos $\breve{\omega}=0$ (o elemento graduante de $\breve{\mathcal{Q}}$ é nulo), ou $U_{i}=\breve{\mathcal{A}}_{0}$, para todo $i \in\{1,2, \ldots, m\}$. Certamente a segunda premissa implica $V_{i}=\mathcal{A}_{0}$, para todo $i \in\{1,2, \ldots, m\}$. Agora, $\breve{\omega}=0$ implica $\breve{\sigma}=\mathrm{id}_{\breve{Q}}$ ou $\breve{\sigma}$ é um automorfismo externo. Para $(x, y) \in \breve{\mathcal{Q}}$, temos

$$
\begin{aligned}
\breve{\sigma}(x, y) & =\breve{\sigma}\left(\left(x_{0}, y_{0}\right)+\left(x_{1}, y_{1}\right)\right) \\
& =\left(x_{0}, y_{0}\right)-\left(x_{1}, y_{1}\right)=\left(x_{0}-x_{1}, y_{0}-y_{1}\right) \\
& =(\sigma(x), \sigma(y))
\end{aligned}
$$

então, $\breve{\sigma}=\operatorname{id}_{\breve{Q}}$ implica $\sigma=\operatorname{id}_{\mathcal{Q}}$. Se $\sigma$ é um automorfismo interno, então $\sigma(a)=\omega a \omega^{-1}$, para todo $a \in \mathcal{Q}$ ( $\omega$ é um elemento graduante de $\mathcal{Q}$ ). Desse modo, definindo $\rho=(\omega, 0) \in \breve{\mathcal{Q}}$, temos

$$
\rho\left(\omega^{-1}, 0\right)=(\omega, 0)\left(\omega^{-1}, 0\right)=\left(\omega \omega^{-1}, 0\right)=(1,0),
$$


isto é, $\rho$ é invertível e $\rho^{-1}=\left(\omega^{-1}, 0\right)$. Além disso, para todo $(x, y) \in \breve{\mathcal{Q}}$,

$$
\begin{aligned}
\rho(x, y) \rho^{-1} & =(\omega, 0)(x, y)\left(\omega^{-1}, 0\right) \\
& =(\omega x, \omega y)\left(\omega^{-1}, 0\right) \\
& =\left(\omega x \omega^{-1}, \omega y \omega^{-1}\right)=(\sigma(x), \sigma(y)) \\
& =\breve{\sigma}(x, y) .
\end{aligned}
$$

Portanto, $\breve{\sigma}$ é um automorfismo interno. As observações anteriores provam que $\breve{\omega}=0$ implica $\breve{\sigma}=\operatorname{id}_{\breve{Q}}$ ou $\breve{\sigma}$ é externo, o que implica $\sigma=\operatorname{id}_{\mathcal{Q}}$ ou $\sigma$ é externo, e concluímos $\omega=0$. Assim, estamos no caso em que vale $\omega=0$ ou $V_{i}=\mathcal{A}_{0}$, para todo $i$. Logo, como $\mathcal{A}$ é um submódulo graduado $d$-superlivre de $\mathcal{Q}$, concluímos que a identidade funcional de (6.48) possui apenas a solução padrão:

$$
\begin{gathered}
E_{i}\left(\bar{x}_{m}^{i}\right)=\sum_{\substack{j \in \mathcal{J} \\
j \neq i}} x_{j} p_{i j}\left(\bar{x}_{m}^{i j}\right)+\lambda_{i}\left(\bar{x}_{m}^{i}\right), \\
F_{j}\left(\bar{x}_{m}^{j}\right)=-\sum_{\substack{i \in \mathcal{I} \\
i \neq j}} p_{i j}\left(\bar{x}_{m}^{i j}\right) x_{i}-\lambda_{j}\left(\bar{x}_{m}^{j}\right),
\end{gathered}
$$

onde $p_{i j}: \hat{V}^{i j} \rightarrow \mathcal{Q}, \lambda_{k}: \hat{V}^{k} \rightarrow \mathcal{C}+\mathcal{C} \omega$, e $\lambda_{k}=0$ se $k \notin \mathcal{I} \cap \mathcal{J}$.

Substituindo na identidade funcional de (6.49), segue

$$
\begin{aligned}
& \sum_{i \in \mathcal{I}} \sum_{\substack{j \in \mathcal{J} \\
j \neq i}} x_{j} p_{i j}\left(\bar{x}_{m}^{i j}\right) \delta\left(x_{i}\right)+\sum_{i \in \mathcal{I}} \lambda_{i}\left(\bar{x}_{m}^{i}\right) \delta\left(x_{i}\right)+\sum_{i \in \mathcal{I}} G_{i}\left(\bar{x}_{m}^{i}\right) x_{i} \\
& -\sum_{j \in \mathcal{J}} \sum_{\substack{i \in \mathcal{I} \\
i \neq j}} \delta\left(x_{j}\right) p_{i j}\left(\bar{x}_{m}^{i j}\right) x_{i}-\sum_{j \in \mathcal{J}} \delta\left(x_{j}\right) \lambda_{j}\left(\bar{x}_{m}^{j}\right)+\sum_{j \in \mathcal{J}} x_{j} H_{j}\left(\bar{x}_{m}^{j}\right)=0
\end{aligned}
$$

Desse modo, como estamos no caso em que vale $\omega=0$ ou $V_{i}=\mathcal{A}_{0}$, para todo $i$, e $\delta$ preserva a

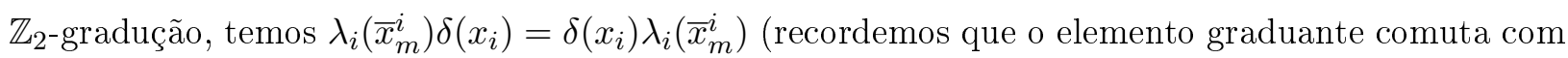
os elementos de grau 0 e anticomuta com os elementos de grau 1), para cada $i \in \mathcal{I} \cap \mathcal{J}$. Portanto, concluímos a validade de

$$
\sum_{i \in \mathcal{I}} \lambda_{i}\left(\bar{x}_{m}^{i}\right) \delta\left(x_{i}\right)-\sum_{j \in \mathcal{J}} \delta\left(x_{j}\right) \lambda_{j}\left(\bar{x}_{m}^{j}\right)=0 .
$$

Logo, rearranjando os somatórios, segue

$$
\sum_{i \in \mathcal{I}}\left[G_{i}\left(\bar{x}_{m}^{i}\right)-\sum_{\substack{j \in \mathcal{J} \\ j \neq i}} \delta\left(x_{j}\right) p_{i j}\left(\bar{x}_{m}^{i j}\right)\right] x_{i}+\sum_{j \in \mathcal{J}} x_{j}\left[H_{j}\left(\bar{x}_{m}^{j}\right)+\sum_{\substack{i \in \mathcal{I} \\ i \neq j}} p_{i j}\left(\bar{x}_{m}^{i j}\right) \delta\left(x_{i}\right)\right]=0 .
$$

de onde obtemos uma nova identidade funcional. Assim, como $\mathcal{A}$ é $d$-superlivre e estamos no caso $\omega=0$ ou $V_{i}=\mathcal{A}_{0}$, para todo $i$, essa identidade funcional possui apenas a solução padrão

$$
G_{i}\left(\bar{x}_{m}^{i}\right)-\sum_{\substack{j \in \mathcal{J} \\ j \neq i}} \delta\left(x_{j}\right) p_{i j}\left(\bar{x}_{m}^{i j}\right)=\sum_{\substack{j \in \mathcal{J} \\ j \neq i}} x_{j} q_{i j}\left(\bar{x}_{m}^{i j}\right)+\mu_{i}\left(\bar{x}_{m}^{i}\right),
$$




$$
H_{j}\left(\bar{x}_{m}^{j}\right)+\sum_{\substack{i \in \mathcal{I} \\ i \neq j}} p_{i j}\left(\bar{x}_{m}^{i j}\right) \delta\left(x_{i}\right)=-\sum_{\substack{i \in \mathcal{I} \\ i \neq j}} q_{i j}\left(\bar{x}_{m}^{i j}\right) x_{i}-\mu_{j}\left(\bar{x}_{m}^{j}\right),
$$

onde $q_{i j}: \hat{V}^{i j} \rightarrow \mathcal{Q}, \mu_{k}: \hat{V}^{k} \rightarrow \mathcal{C}+\mathcal{C} \omega$.

Agora, defina $\breve{p}_{i j}: \hat{U}^{i j} \rightarrow \breve{\mathcal{Q}}$ e $\breve{\lambda}_{k}: \hat{U}^{k} \rightarrow \breve{\mathcal{C}}+\breve{\mathcal{C}} \breve{\omega}$ por

$$
\begin{gathered}
\left.\breve{p}_{i j}\left(\bar{u}_{m}^{i j}\right)=\left(p_{i j}(\overline{(x})_{m}^{i j}\right), q_{i j}\left(\bar{x}_{m}^{i j}\right)\right), \\
\breve{\lambda}_{k}\left(\bar{u}_{m}^{k}\right)=\left(\lambda_{k}\left(\bar{x}_{m}^{k}\right), \mu_{k}\left(\bar{x}_{m}^{k}\right)\right),
\end{gathered}
$$

para todos $\bar{u}_{m}^{i j} \in \hat{U}^{i j}, \bar{u}_{m}^{k} \in \hat{U}^{k}$. Observe que se $k \notin \mathcal{I} \cap \mathcal{J}, \breve{\lambda}_{k}=(0,0)$. Além disso, $\breve{\lambda}_{k}\left(\hat{U}^{k}\right) \subseteq \breve{\mathcal{C}}+\breve{\mathcal{C}} \omega$. De fato, $\breve{\lambda}_{k}\left(\hat{U}^{k}\right) \subseteq(\mathcal{C}+\mathcal{C} \omega) \times(\mathcal{C}+\mathcal{C} \omega) \subseteq \breve{\mathcal{C}}+\breve{\mathcal{C}} \omega$, pois

$$
\begin{aligned}
(\mathcal{C}+\mathcal{C} \omega) \times(\mathcal{C}+\mathcal{C} \omega) \ni(a+b \omega, c+d \omega) & =(a, c)+(b \omega, d \omega) \\
& =(a, c)+(b, d)(\omega, 0) \\
& =(a, c)+(b, d) \breve{\omega} \in(\mathcal{C} \times \mathcal{C})+(\mathcal{C} \times \mathcal{C}) \breve{\omega}
\end{aligned}
$$

Observamos também que um elemento graduante de $\breve{\mathcal{Q}}$ é $\breve{\omega}=(\omega, 0)$. Para tanto, já sabemos que se $\breve{\omega}=0$, então, $\omega=0$ e logo $\breve{\omega}=(0,0)=(\omega, 0)$. Se $\breve{\omega} \neq 0$, então, $\breve{\sigma}$ é um automorfismo interno, isto é, existe $\rho=(a, b) \in \breve{\mathcal{Q}}$ invertível, tal que

$$
\breve{\sigma}(x, y)=\rho(x, y) \rho^{-1}, \text { para todo }(x, y) \in \breve{\mathcal{Q}} \text {. }
$$

Fazendo alguns cálculos, sendo $(1,0)$ a identidade de $\breve{\mathcal{Q}}$, concluímos $\rho^{-1}=\left(a^{-1},-a^{-1} b a^{-1}\right)$, de onde segue

$$
\begin{aligned}
\breve{\sigma}(x, y) & =(a, b)(x, y)\left(a^{-1},-a^{-1} b a^{-1}\right) \\
& =(a x, a y+b x)\left(a^{-1},-a^{-1} b a^{-1}\right) \\
& =\left(a x a^{-1},-a x a^{-1} b a^{-1}+a y a^{-1}+b x a^{-1}\right)
\end{aligned}
$$

para todo $(x, y) \in \breve{\mathcal{Q}}$. Entretanto, como $\breve{\sigma}(x, y)=(\sigma(x), \sigma(y))$, podemos concluir $\sigma(x)=a x a^{-1}$, para todo $x \in \mathcal{Q}$. Logo, $\sigma$ é automorfismo interno e $0 \neq a$ é um elemento graduante de $\mathcal{Q}$. Assim, como $\rho$ é um elemento graduante de $\breve{\mathcal{Q}}$, temos também que $\rho$ comuta com os elementos de $\breve{\mathcal{Q}}_{0}$ e anticomuta com os elementos de $\breve{\mathcal{Q}}_{1}$, ou seja, $(a, b)\left(x_{0}, y_{0}\right)=\left(x_{0}, y_{0}\right)(a, b)$ e $(a, b)\left(x_{1}, y_{1}\right)=$ $-\left(x_{1}, y_{1}\right)(a, b)$. Estas igualdades implicam $a x_{0}=x_{0} a, b x_{0}=x_{0} b, a x_{1}=-x_{1} a$ e $b x_{1}=-x_{1} b$, para todos $x_{0}, y_{0} \in \mathcal{Q}_{0}, x_{1}, y_{1} \in \mathcal{Q}_{1}$. Desse modo, ax $=\sigma(x) a$ e $b x=\sigma(x) b$, para todo $x \in \mathcal{Q}$ $\left(\sigma(x)=x_{0}-x_{1}\right)$, o que nos permite concluir

$$
\begin{aligned}
\breve{\sigma}(x, y) & =\left(a x a^{-1},-a x a^{-1} b a^{-1}+a y a^{-1}+b x a^{-1}\right) \\
& =\left(a x a^{-1},-\sigma(x) b a^{-1}+a y a^{-1}+\sigma(x) b a^{-1}\right) \\
& =\left(a x a^{-1}, a y a^{-1}\right) \\
& =(a, 0)(x, y)\left(a^{-1}, 0\right),
\end{aligned}
$$

para todo $(x, y) \in \breve{\mathcal{Q}}$. Portanto, podemos tomar como um elemento graduante de $\breve{\mathcal{Q}}, \breve{\omega}=(\omega, 0)$, onde $\omega$ é um elemento graduante de $\mathcal{Q}$. Finalmente, pelo Lema 6.1 .2 , já sabemos que vale $\mathcal{C} \times \mathcal{C}=\breve{\mathcal{C}}$, o que prova aquilo que queríamos. 
Definidas as aplicações $\breve{p}_{i j}$ e $\breve{\lambda}_{k}$, seguem as igualdades

$$
\begin{aligned}
\breve{E}_{i}\left(\bar{u}_{m}^{i}\right) & =\left(E_{i}\left(\bar{x}_{m}^{i}\right), G_{i}\left(\bar{x}_{m}^{i}\right)\right) \\
& =\left(\sum_{\substack{j \in \mathcal{J} \\
j \neq i}} x_{j} p_{i j}\left(\bar{x}_{m}^{i j}\right)+\lambda_{i}\left(\bar{x}_{m}^{i}\right), \sum_{\substack{j \in \mathcal{J} \\
j \neq i}}\left[\delta\left(x_{j}\right) p_{i j}\left(\bar{x}_{m}^{i j}\right)+x_{j} q_{i j}\left(\bar{x}_{m}^{i j}\right)\right]+\mu_{i}\left(\bar{x}_{m}^{i}\right)\right) \\
& =\left(\sum_{\substack{j \in \mathcal{J} \\
j \neq i}} x_{j} p_{i j}\left(\bar{x}_{m}^{i j}\right), \sum_{\substack{j \in \mathcal{J} \\
j \neq i}} \delta\left(x_{j}\right) p_{i j}\left(\bar{x}_{m}^{i j}\right)+x_{j} q_{i j}\left(\bar{x}_{m}^{i j}\right)\right)+\left(\lambda_{i}\left(\bar{x}_{m}^{i}\right), \mu_{i}\left(\bar{x}_{m}^{i}\right)\right) \\
& =\sum_{\substack{j \in \mathcal{J} \\
j \neq i}}\left(x_{j} p_{i j}\left(\bar{x}_{m}^{i j}\right), \delta\left(x_{j}\right) p_{i j}\left(\bar{x}_{m}^{i j}\right)+x_{j} q_{i j}\left(\bar{x}_{m}^{i j}\right)\right)+\breve{\lambda}_{i}\left(\bar{u}_{m}^{i}\right) \\
& =\sum_{\substack{j \in \mathcal{J} \\
j \neq i}}\left(x_{j}, \delta\left(x_{j}\right)\right)\left(p_{i j}\left(\bar{x}_{m}^{i j}\right), q_{i j}\left(\bar{x}_{m}^{i j}\right)\right)+\breve{\lambda}_{i}\left(\bar{u}_{m}^{i}\right) \\
& =\sum_{\substack{j \in \mathcal{J} \\
j \neq i}} u_{j} \breve{p}_{i j}\left(\bar{u}_{m}^{i j}\right)+\breve{\lambda}_{i}\left(\bar{u}_{m}^{i}\right) .
\end{aligned}
$$

Analogamente,

$$
\begin{aligned}
\breve{F}_{j}\left(\bar{u}_{m}^{j}\right)= & \left(F_{j}\left(\bar{x}_{m}^{j}\right), H_{j}\left(\bar{x}_{m}^{j}\right)\right) \\
= & \left(-\sum_{\substack{i \in \mathcal{I} \\
i \neq j}} p_{i j}\left(\bar{x}_{m}^{i j}\right) x_{i}-\lambda_{j}\left(\bar{x}_{m}^{j}\right),-\sum_{\substack{i \in \mathcal{I} \\
i \neq j}}\left[p_{i j}\left(\bar{x}_{m}^{i j}\right) \delta\left(x_{i}\right)+q_{i j}\left(\bar{x}_{m}^{i j}\right) x_{i}\right]-\mu_{j}\left(\bar{x}_{m}^{j}\right)\right) \\
= & \left(-\sum_{\substack{i \in \mathcal{I} \\
i \neq j}} p_{i j}\left(\bar{x}_{m}^{i j}\right) x_{i},-\sum_{\substack{i \in \mathcal{I} \\
i \neq j}}\left[p_{i j}\left(\bar{x}_{m}^{i j}\right) \delta\left(x_{i}\right)+q_{i j}\left(\bar{x}_{m}^{i j}\right) x_{i}\right]\right)-\left(\lambda_{j}\left(\bar{x}_{m}^{j}\right), \mu_{j}\left(\bar{x}_{m}^{j}\right)\right) \\
= & -\sum_{\substack{i \in \mathcal{I} \\
i \neq j}}\left(p_{i j}\left(\bar{x}_{m}^{i j}\right) x_{i}, p_{i j}\left(\bar{x}_{m}^{i j}\right) \delta\left(x_{i}\right)+q_{i j}\left(\bar{x}_{m}^{i j}\right) x_{i}\right)-\breve{\lambda}_{j}\left(\bar{u}_{m}^{j}\right) \\
= & -\sum_{\substack{i \in \mathcal{I} \\
i \neq j}}\left(p_{i j}\left(\bar{x}_{m}^{i j}\right), q_{i j}\left(\bar{x}_{m}^{i j}\right)\right)\left(x_{i}, \delta\left(x_{i}\right)\right)-\breve{\lambda}_{j}\left(\bar{u}_{m}^{j}\right) \\
= & -\sum_{\substack{i \in \mathcal{I} \\
i \neq j}} \breve{p}_{i j}\left(\bar{u}_{m}^{i j}\right) u_{i}-\breve{\lambda}_{j}\left(\bar{u}_{m}^{j}\right) .
\end{aligned}
$$

Portanto, neste primeiro caso, a I.F. em (6.47) possui apenas a solução padrão. Prosseguimos, então, para o segundo caso. Suponha $\breve{\omega} \neq 0$ e que exista $i \in\{1,2, \ldots, m\}$, tal que $U_{i}=\breve{\mathcal{A}}_{1}$. Sabemos, que vale $\omega \neq 0(\breve{\omega}=(\omega, 0))$ e que existe $i \in\{1,2, \ldots, m\}$, tal que $V_{i}=\mathcal{A}_{1}$. Desse modo, retornamos às identidades funcionais de (6.48) e de (6.49). Neste caso, como $\mathcal{A}$ é $d$-livre em $\mathcal{Q}$, a I.F. em (6.48) possui apenas a seguinte solução padrão

$$
E_{i}\left(\bar{x}_{m}^{i}\right)=\sum_{\substack{j \in \mathcal{J} \\ j \neq i}} x_{j} p_{i j}\left(\bar{x}_{m}^{i j}\right)+\lambda_{i}\left(\bar{x}_{m}^{i}\right)+\mu_{i}\left(\bar{x}_{m}^{i}\right) \omega
$$




$$
F_{j}\left(\bar{x}_{m}^{j}\right)=-\sum_{\substack{i \in \mathcal{I} \\ i \neq j}} p_{i j}\left(\bar{x}_{m}^{i j}\right) x_{i}-\lambda_{j}\left(\bar{x}_{m}^{j}\right)-\epsilon_{j} \mu_{j}\left(\bar{x}_{m}^{j}\right) \omega
$$

onde $p_{i j}: \hat{V}^{i j} \rightarrow \mathcal{Q}, \lambda_{k}, \mu_{k}: \hat{V}^{k} \rightarrow \mathcal{C}, \lambda_{k}=\mu_{k}=0$, se $k \notin \mathcal{I} \cap \mathcal{J}, \epsilon_{j}=1$, se $V_{j}=\mathcal{A}_{0}$, e $\epsilon_{j}=-1$, se $V_{j}=\mathcal{A}_{1}$.

Substituindo em (6.49), obtemos

$$
\begin{aligned}
\sum_{i \in \mathcal{I}} \sum_{\substack{j \in \mathcal{J} \\
j \neq i}} x_{j} p_{i j}\left(\bar{x}_{m}^{i j}\right) \delta\left(x_{i}\right)+\sum_{i \in \mathcal{I}} \lambda_{i}\left(\bar{x}_{m}^{i}\right) \delta\left(x_{i}\right)+\sum_{i \in \mathcal{I}} \mu_{i}\left(\bar{x}_{m}^{i}\right) \omega \delta\left(x_{i}\right)+\sum_{i \in \mathcal{I}} G_{i}\left(\bar{x}_{m}^{i}\right) x_{i} \\
\quad-\sum_{j \in \mathcal{J}} \sum_{\substack{i \in \mathcal{I} \\
i \neq j}} \delta\left(x_{j}\right) p_{i j}\left(\bar{x}_{m}^{i j}\right) x_{i}-\sum_{j \in \mathcal{J}} \delta\left(x_{j}\right) \lambda_{j}\left(\bar{x}_{m}^{j}\right)-\sum_{j \in \mathcal{J}} \delta\left(x_{j}\right) \epsilon_{j} \mu_{j}\left(\bar{x}_{m}^{j}\right) \omega+\sum_{j \in \mathcal{J}} x_{j} H_{j}\left(\bar{x}_{m}^{j}\right)=0 .
\end{aligned}
$$

Agora, como $\lambda_{k}\left(\bar{x}_{m}^{k}\right) \in \mathcal{C}$ e $\lambda_{k}=0$, se $k \notin \mathcal{I} \cap \mathcal{J}$, temos

$$
\sum_{i \in \mathcal{I}} \lambda_{i}\left(\bar{x}_{m}^{i}\right) \delta\left(x_{i}\right)-\sum_{j \in \mathcal{J}} \delta\left(x_{j}\right) \lambda_{j}\left(\bar{x}_{m}^{j}\right)=0
$$

Além disso, $\mu_{k}\left(\bar{x}_{m}^{k}\right) \in \mathcal{C}$ e $\mu_{k}=0$, se $k \notin \mathcal{I} \cap \mathcal{J}$. Se $V_{k}=\mathcal{A}_{0}$, então, como $\delta$ é uma aplicação graduada, segue $\delta\left(x_{k}\right) \in \mathcal{Q}_{0}$ e $\epsilon_{k}=1$. Logo,

$$
\mu_{k}\left(\bar{x}_{m}^{k}\right) \omega \delta\left(x_{k}\right)-\delta\left(x_{k}\right) \epsilon_{k} \mu_{k}\left(\bar{x}_{m}^{k}\right)=0
$$

e se $V_{k}=\mathcal{A}_{1}$, então, $\delta\left(x_{k}\right) \in \mathcal{Q}_{1}$ e $\epsilon_{k}=-1$. Assim, novamente temos a igualdade anterior. Estas observações implicam

$$
\sum_{i \in \mathcal{I}} \mu_{i}\left(\bar{x}_{m}^{i}\right) \omega \delta\left(x_{i}\right)-\sum_{j \in \mathcal{J}} \delta\left(x_{j}\right) \epsilon_{j} \mu_{j}\left(\bar{x}_{m}^{j}\right) \omega=0 .
$$

Reescrevendo os somatórios, obtemos a seguinte I.F.

$$
\begin{aligned}
\sum_{i \in \mathcal{I}}\left[G_{i}\left(\bar{x}_{m}^{i}\right)\right. & \left.-\sum_{\substack{j \in \mathcal{J} \\
j \neq i}} \delta\left(x_{j}\right) p_{i j}\left(\bar{x}_{m}^{i j}\right)\right] x_{i}+ \\
& +\sum_{j \in \mathcal{J}} x_{j}\left[H_{j}\left(\bar{x}_{m}^{j}\right)+\sum_{\substack{i \in \mathcal{I} \\
i \neq j}} p_{i j}\left(\bar{x}_{m}^{i j}\right) \delta\left(x_{i}\right)\right]=0 .
\end{aligned}
$$

Assim, como $\mathcal{A}$ é $d$-superlivre em $\mathcal{Q}$, concluímos que a última identidade funcional possui apenas a solução padrão.

$$
\begin{aligned}
G_{i}\left(\bar{x}_{m}^{i}\right)-\sum_{\substack{j \in \mathcal{J} \\
j \neq i}} \delta\left(x_{j}\right) p_{i j}\left(\bar{x}_{m}^{i j}\right)=\sum_{\substack{j \in \mathcal{J} \\
j \neq i}} x_{j} q_{i j}\left(\bar{x}_{m}^{i j}\right)+\alpha_{i}\left(\bar{x}_{m}^{i}\right)+\beta_{i}\left(\bar{x}_{m}^{i}\right) \omega, \\
H_{j}\left(\bar{x}_{m}^{j}\right)+\sum_{\substack{i \in \mathcal{I} \\
i \neq j}} p_{i j}\left(\bar{x}_{m}^{i j}\right) \delta\left(x_{i}\right)=-\sum_{\substack{i \in \mathcal{I} \\
i \neq j}} q_{i j}\left(\bar{x}_{m}^{i j}\right) x_{i}-\alpha_{j}\left(\bar{x}_{m}^{j}\right)-\epsilon_{j} \beta_{j}\left(\bar{x}_{m}^{j}\right) \omega,
\end{aligned}
$$

onde $q_{i j}: \hat{V}^{i j} \rightarrow \mathcal{Q}, \alpha_{k}, \beta_{k}: \hat{V}^{k} \rightarrow \mathcal{C}, \alpha_{k}=\beta_{k}=0$, se $k \notin \mathcal{I} \cap \mathcal{J}$. 
Prosseguindo, defina $\breve{p}_{i j}: \hat{U}^{i j} \rightarrow \breve{\mathcal{Q}}, \breve{\lambda}_{k}, \breve{\mu}_{k}: \hat{U}^{k} \rightarrow \breve{\mathcal{C}}$ por

$$
\begin{aligned}
\breve{p}_{i j}\left(\bar{u}_{m}^{i j}\right) & =\left(p_{i j}\left((\bar{x})_{m}^{i j}\right), q_{i j}\left(\bar{x}_{m}^{i j}\right)\right), \\
\breve{\lambda}_{k}\left(\bar{u}_{m}^{k}\right) & =\left(\lambda_{k}\left(\bar{x}_{m}^{k}\right), \alpha_{k}\left(\bar{x}_{m}^{k}\right)\right), \\
\breve{\mu}_{k}\left(\bar{u}_{m}^{k}\right) & =\left(\epsilon_{k} \mu_{k}\left(\bar{x}_{m}^{k}\right), \epsilon_{k} \beta_{k}\left(\bar{x}_{m}^{k}\right)\right)
\end{aligned}
$$

para todos $\bar{u}_{m}^{i j} \in \hat{U}^{i j}, \bar{u}_{m}^{k} \in \hat{U}^{k}$. Observe que, se $k \notin \mathcal{I} \cap \mathcal{J}$, então, $\breve{\lambda}_{k}=\breve{\mu}_{k}=(0,0)$. Além disso, $\breve{\lambda}_{k}\left(\hat{U}^{k}\right), \breve{\mu}_{k}\left(\hat{U}^{k}\right) \subseteq \breve{\mathcal{C}}=\mathcal{C} \times \mathcal{C}$. Finalmente, segue

$$
\begin{aligned}
\breve{E}_{i}\left(\bar{u}_{m}^{i}\right)= & \left(E_{i}\left(\bar{x}_{m}^{i}\right), G_{i}\left(\bar{x}_{m}^{i}\right)\right) \\
= & \left(\sum_{\substack{j \in \mathcal{J} \\
j \neq i}} x_{j} p_{i j}\left(\bar{x}_{m}^{i j}\right)+\lambda_{i}\left(\bar{x}_{m}^{i}\right)+\epsilon_{i} \mu_{i}\left(\bar{x}_{m}^{i}\right) \omega,\right. \\
\left.\left.\sum_{\substack{j \in \mathcal{J} \\
j \neq i}}\left[\delta\left(x_{j}\right) p_{i j}\left(\bar{x}_{m}^{i j}\right)+x_{j} q_{i j}\left(\bar{x}_{m}^{i j}\right)\right]+\alpha_{i}\left(\bar{x}_{m}^{i}\right)+\epsilon_{i} \beta_{i}\left(\bar{x}_{m}^{i}\right) \omega\right)\right) & \left(\sum_{\substack{j \in \mathcal{J} \\
j \neq i}} x_{j} p_{i j}\left(\bar{x}_{m}^{i j}\right), \sum_{j \in \mathcal{J}} \delta\left(x_{j}\right) p_{i j}\left(\bar{x}_{m}^{i j}\right)+x_{j} q_{i j}\left(\bar{x}_{m}^{i j}\right)\right)+\left(\lambda_{i}\left(\bar{x}_{m}^{i}\right), \alpha_{i}\left(\bar{x}_{m}^{i}\right)\right)+ \\
& +\left(\epsilon_{i} \mu_{i}\left(\bar{x}_{m}^{i}\right), \epsilon_{i} \beta_{i}\left(\bar{x}_{m}^{i}\right)\right)(\omega, 0) \\
= & \sum_{\substack{j \in \mathcal{J} \\
j \neq i}}\left(x_{j} p_{i j}\left(\bar{x}_{m}^{i j}\right), \delta\left(x_{j}\right) p_{i j}\left(\bar{x}_{m}^{i j}\right)+x_{j} q_{i j}\left(\bar{x}_{m}^{i j}\right)\right)+\breve{\lambda}_{i}\left(\bar{u}_{m}^{i}\right)+\breve{\mu}_{i}\left(\bar{u}_{m}^{i}\right) \breve{\omega} \\
= & \sum_{\substack{j \in \mathcal{J} \\
j \neq i}}\left(x_{j}, \delta\left(x_{j}\right)\right)\left(p_{i j}\left(\bar{x}_{m}^{i j}\right), q_{i j}\left(\bar{x}_{m}^{i j}\right)\right)+\breve{\lambda}_{i}\left(\bar{u}_{m}^{i}\right)+\breve{\mu}_{i}\left(\bar{u}_{m}^{i}\right) \breve{\omega} \\
= & \sum_{\substack{j \in \mathcal{J} \\
j \neq i}} u_{j} \breve{p}_{i j}\left(\bar{u}_{m}^{i j}\right)+\breve{\lambda}_{i}\left(\bar{u}_{m}^{i}\right)+\breve{\mu}_{i}\left(\bar{u}_{m}^{i}\right) \breve{\omega} .
\end{aligned}
$$


Analogamente,

$$
\begin{aligned}
& \breve{F}_{j}\left(\bar{u}_{m}^{j}\right)=\left(F_{j}\left(\bar{x}_{m}^{j}\right), H_{j}\left(\bar{x}_{m}^{j}\right)\right) \\
& =\left(-\sum_{\substack{i \in \mathcal{I} \\
i \neq j}} p_{i j}\left(\bar{x}_{m}^{i j}\right) x_{i}-\lambda_{j}\left(\bar{x}_{m}^{j}\right)-\epsilon_{j} \mu_{j}\left(\bar{x}_{m}^{j}\right) \omega,\right. \\
& \left.-\sum_{\substack{i \in \mathcal{I} \\
i \neq j}}\left[p_{i j}\left(\bar{x}_{m}^{i j}\right) \delta\left(x_{i}\right)+q_{i j}\left(\bar{x}_{m}^{i j}\right) x_{i}\right]-\alpha_{j}\left(\bar{x}_{m}^{j}\right)-\epsilon_{j} \beta_{j}\left(\bar{x}_{m}^{j}\right) \omega\right) \\
& =\left(-\sum_{\substack{i \in \mathcal{I} \\
i \neq j}} p_{i j}\left(\bar{x}_{m}^{i j}\right) x_{i},-\sum_{\substack{i \in \mathcal{I} \\
i \neq j}}\left[p_{i j}\left(\bar{x}_{m}^{i j}\right) \delta\left(x_{i}\right)+q_{i j}\left(\bar{x}_{m}^{i j}\right) x_{i}\right]\right)-\left(\lambda_{j}\left(\bar{x}_{m}^{j}\right), \alpha_{j}\left(\bar{x}_{m}^{j}\right)\right) \\
& -\left(\epsilon_{j} \mu_{j}\left(\bar{x}_{m}^{j}\right), \epsilon_{j} \beta_{j}\left(\bar{x}_{m}^{j}\right)\right)(\omega, 0) \\
& =-\sum_{i \in \mathcal{I}}\left(p_{i j}\left(\bar{x}_{m}^{i j}\right) x_{i}, p_{i j}\left(\bar{x}_{m}^{i j}\right) \delta\left(x_{i}\right)+q_{i j}\left(\bar{x}_{m}^{i j}\right) x_{i}\right)-\breve{\lambda}_{j}\left(\bar{u}_{m}^{j}\right)-\breve{\mu}_{j}\left(\bar{u}_{m}^{j}\right) \breve{\omega} \\
& =-\sum_{i \in \mathcal{I}}^{i \neq j}\left(p_{i j}\left(\bar{x}_{m}^{i j}\right), q_{i j}\left(\bar{x}_{m}^{i j}\right)\right)\left(x_{i}, \delta\left(x_{i}\right)\right)-\breve{\lambda}_{j}\left(\bar{u}_{m}^{j}\right)-\breve{\mu}_{j}\left(\bar{u}_{m}^{j}\right) \breve{\omega} \\
& =-\sum_{\substack{i \in \mathcal{I} \\
i \neq j}}^{i \neq j} \breve{p}_{i j}\left(\bar{u}_{m}^{i j}\right) u_{i}-\breve{\lambda}_{j}\left(\bar{u}_{m}^{j}\right)-\breve{\mu}_{j}\left(\bar{u}_{m}^{j}\right) \breve{\omega} .
\end{aligned}
$$

Portanto, neste segundo caso, a I.F. de (6.47) também possui apenas a solução padrão.

Finalmente, estamos em condições de provar o seguinte

Corolário 6.3.1. Seja $\mathcal{Q}=\mathcal{Q}_{0} \oplus \mathcal{Q}_{1}$ uma superálgebra com identidade e centro $\mathcal{C}=\mathcal{C}_{0} \oplus \mathcal{C}_{1}$. Se $\mathcal{A}=\mathcal{A}_{0} \oplus \mathcal{A}_{1}$ é uma subálgebra de Jordan graduada 4-superlivre de $\mathcal{Q}$, então, toda superderivação de Jordan de grau $0, \delta: \mathcal{A} \rightarrow \mathcal{Q}$, é uma superderivação de grau 0.

Demonstração. Seja $\delta: \mathcal{A} \rightarrow \mathcal{Q}$ uma superderivação de Jordan de grau 0. Considere $\breve{\mathcal{Q}}=\mathcal{Q} \times \mathcal{Q}$ a superálgebra como no Lema 6.3.1. Defina $\alpha: \mathcal{A} \rightarrow \breve{\mathcal{Q}}$, tal que $\alpha(x)=(x, \delta(x))$, para todo $x \in \mathcal{A}$. Vamos provar que $\alpha$ é um superhomomorfismo de Jordan.

(i) $\alpha$ é $\Phi$-linear:

$$
\begin{aligned}
\alpha(k x+y) & =(k x+y, \delta(k x+y)) \\
& =(k x, k \delta(x))+(y,+\delta(y)) \\
& =k \alpha(x)+\alpha(y) .
\end{aligned}
$$

(ii) $\alpha$ preserva a $\mathbb{Z}_{2}$-graduação:

- $x_{0} \in \mathcal{A}_{0}$ implica que $\alpha\left(x_{0}\right)=\left(x_{0}, \delta\left(x_{0}\right)\right) \in \mathcal{Q}_{0} \times \mathcal{Q}_{0}=\breve{\mathcal{Q}}_{0}$;

- $x_{1} \in \mathcal{A}_{1}$ implica que $\alpha\left(x_{1}\right)=\left(x_{1}, \delta\left(x_{1}\right)\right) \in \mathcal{Q}_{1} \times \mathcal{Q}_{1}=\breve{\mathcal{Q}}_{1}$. 
(iii) Para todos $x, y \in \mathcal{A}_{0} \cup \mathcal{A}_{1}$, temos

$$
\begin{aligned}
\alpha\left(x \circ_{s} y\right)= & \left(x \circ_{s} y, \delta\left(x \circ_{s} y\right)\right) \\
= & \left(\frac{1}{2} x y+\frac{1}{2}(-1)^{|x||y|} y x, \delta(x) \circ_{s} y+x \circ_{s} \delta(y)\right) \\
= & \left(\frac{1}{2} x y+\frac{1}{2}(-1)^{|x||y|} y x, \frac{1}{2} \delta(x) y+\frac{1}{2}(-1)^{|\delta(x)||y|} y \delta(x)+\frac{1}{2} x \delta(y)+\right. \\
& \left.+\frac{1}{2}(-1)^{|x||\delta(y)|} \delta(y) x\right) \\
= & \frac{1}{2}\left(x y+(-1)^{|x||y|} y x, \delta(x) y+(-1)^{|x||y|} y \delta(x)+x \delta(y)+\right. \\
& \left.+(-1)^{|x||y|} \delta(y) x\right) \\
= & \frac{1}{2}(x y, \delta(x) y+x \delta(y))+\frac{1}{2}(-1)^{|x||y|}(y x, y \delta(x)+\delta(x) y) \\
= & \frac{1}{2}(x, \delta(x))(y, \delta(y))+\frac{1}{2}(-1)^{|(x, \delta(x))||(y, \delta(y))|}(y, \delta(y))(x, \delta(x)) \\
= & (x, \delta(x)) \circ_{s}(y, \delta(y)) \\
= & \alpha(x) \circ_{s} \alpha(y) .
\end{aligned}
$$

Aplicando o Lema 6.3.1, como $\delta$ é uma superderivação de Jordan de grau 0 , temos que $\breve{\mathcal{A}}=$ $\{(x, \delta(x)) \mid x \in \mathcal{A}\}=\alpha(\mathcal{A})$ é um $\Phi$-submódulo graduado 4-superlivre de $\breve{\mathcal{Q}}$, e como $\alpha$ é um superhomomorfismo de Jordan, segue do Teorema 6.3.1, que $\alpha$ é a soma direta de um superhomomorfismo com um superantihomomorfismo. Desse modo, existe $\epsilon \in \breve{\mathcal{C}}$ um idempotente, tal que $x \mapsto \epsilon \alpha(x)$ é um superhomomorfismo, $x \mapsto(1-\epsilon) \alpha(x)$ é um superantihomomorfismo, e

$$
\alpha(x y)=\epsilon \alpha(x) \alpha(y)+(-1)^{|x||y|}(1-\epsilon) \alpha(y) \alpha(x),
$$

para todos $x, y \in \mathcal{A}_{0} \cup \mathcal{A}_{1}$.

Seja $\epsilon^{\prime}=(1-\epsilon)$, vamos provar que vale $\epsilon^{\prime}=(\omega, 0)$, onde $\omega$ é um idempotente central de $\mathcal{Q}$. Note que, como $\epsilon^{\prime}=(a, b) \in \breve{\mathcal{Q}}$, temos que para todo $(c, d) \in \breve{\mathcal{Q}}$, segue $(a, b)(c, d)=(c, d)(a, b)$, isto é, $(a c, a d+b c)=(c a, c b+d a)$, de onde concluímos $a \in \mathcal{C}=Z(\mathcal{Q})$. Desse modo, $b c=c b$, e portanto também temos $b \in \mathcal{C}$. Além disso, como $\epsilon^{\prime}$ é idempotente, segue $(a, b)(a, b)=(a, b)$, ou seja, $\left(a^{2}, a b+b a\right)=(a, b)$. Logo, $a^{2}=a$, ou seja, $a$ é um idempotente, e $2 a b=b$ implica $a b=2 a^{2} b=2 a b$, isto é, $a b=0$, e consequentemente $b=0$. Portanto, $\epsilon^{\prime}$ tem a forma desejada.

Dados $x, y \in \mathcal{A}_{0} \cup \mathcal{A}_{1}$, temos $\epsilon^{\prime} \alpha(x y)=(-1)^{|x||y|} \epsilon^{\prime} \alpha(y) \alpha(x)$, o que nos permite concluir $\omega x y=$ $(-1)^{|x||y|} \omega y x$, e nos conduz à seguinte I.F.

$$
\omega x y-(-1)^{|x||y|} \omega y x=0
$$

Como $\mathcal{A}$ é 4 -superlivre, então, $\mathcal{A}$ é 2-superlivre, e pelo Lema 6.2.1, segue $\omega=0$. Portanto, $\epsilon^{\prime}=(0,0)$. Desse modo, temos $\epsilon=(1,0)$ (o elemento identidade de $\breve{\mathcal{Q}}$ ) e $\alpha$ é um superhomomorfismo. Portanto, para $x, y \in \mathcal{A}_{0} \cup \mathcal{A}_{1}$, são válidas

$$
\begin{aligned}
\alpha(x y) & =\alpha(x) \alpha(y) \\
(x y, \delta(x y)) & =(x, \delta(x))(y, \delta(y)) \\
(x y, \delta(x y)) & =(x y, x \delta(y)+\delta(x) y) .
\end{aligned}
$$

Logo, $\delta(x y)=\delta(x) y+(-1)^{0|x|} x \delta(y)$, ou seja, $\delta$ é uma superderivação de grau 0. 


\section{Referências Bibliográficas}

[1] G. Ancochea. Le théorem de von staudt en géometrie projective quaternionienne. J. Reine Angew. Math., 184:192-198, 1942.

[2] G. Ancochea. On semi-automorphisms of division algebras. Ann. of Math., 48:147-154, 1947.

[3] K. I. Beidar, M. Brešar, and M. A. Chebotar. Jordan superhomomorphisms. Comm. Algebra, 31(2):633-644, 2003.

[4] K. I. Beidar and M. A. Chebotar. On functional identities and d-free subsets of rings. i. Comm. Algebra, 28(8):3925-3951, 2000.

[5] K. I. Beidar and M. A. Chebotar. On functional identities and d-free subsets of rings. ii. Comm. Algebra, 28(8):3953-3972, 2000.

[6] K. I. Beidar, A. V. Mikhalev, and M. A. Chebotar. Functional identities in rings and their applications. Russian Math. Surveys, 59(3):403-428, 2004.

[7] M. Brešar. Jordan derivations on semiprime rings. Proc. Amer. Math. Soc., 104(4):1003-1006, 1988.

[8] M. Brešar, W. S. Martindale, 3rd, and M. A. Chebotar. Functional Identities. Birkhauser Verlag, first edition, 2007.

[9] J. M. Cusack. Jordan derivations on rings. Proceedings of the American Mathematical Society, $53(2): 321-324,1975$.

[10] M. Fošner. Jordan superderivations. Comm. Algebra, 31(9):4533-4543, 2003.

[11] M. Fošner. Jordan superderivations ii. Int. J. Math. Sci., 41-44:2357-2369, 2004.

[12] C. Gómez-Ambrosi, J. Laliena, and I. P. Shestakov. On the lie structure of the skew elements of a prime superalgebra with superinvolution. Comm. Algebra, 2:3277-3291, 2000.

[13] C. Gómez-Ambrosi and F. Montaner. On herstein's constructions relating jordan and associative superalgebras. Comm. Algebra, 28:3743-3762, 2000.

[14] C. Gómez-Ambrosi and I. P. Shestakov. On the lie structure of the skew elements of a simple superalgebra with superinvolution. J. Algebra, 208:43-71, 1998.

[15] I. N. Herstein. Jordan homomorphisms. Trans. Amer. Math. Soc., 81:331-341, 1956.

[16] I. N. Herstein. Jordan derivations of prime rings. Proc. Amer. Math. Soc., 8:1104-1110, 1957. 
[17] I. N. Herstein. Topics in ring theory. The University of Chicago Press, 1969.

[18] I. N. Herstein. Rings with Involution. The University of Chicago Press, 1976.

[19] L. K. Hua. On the automorphisms of a field. Proc. Nat. Acad. Sci. U.S.A., 35:386-389, 1949.

[20] N. Jacobson and C. E. Rickart. Jordan homomorphisms of rings. Trans. Amer. Math. Soc., 69:479-502, 1950.

[21] J. Jolie. Supersimetria. Scientific American Brasil, 3:60-67, 2002.

[22] V. G. Kac. Classification of simple $z$-graded lie superalgebras and simple jordan superalgebras. Comm. Algebra, 13:1375-1400, 1977.

[23] V. G. Kac. Lie superalgebras. Advances in Mathematics, 26:8-96, 1977.

[24] I. Kaplansky. Semi-automorphisms of rings. Duke Math. J., 14:521-527, 1947.

[25] W. S. Martindale, 3rd, and W. E. Baxter. Jordan homomorphisms of semiprime rings. J. Algebra, 56:457-471, 1979.

[26] N. H. McCoy. The theory of rings. Chelsea Publishing Company, 1973.

[27] K. McCrimmon. A taste of Jordan Algebras. Springer, first edition, 2003.

[28] F. Montaner. On the lie structure of associative superalgebras. Comm. Algebra, 26:2337-2349, 1998.

[29] S. Montgomery. Constructing simple lie superalgebras from associative graded algebras. J. Algebra, 195:558-579, 1997.

[30] I. P. Shestakov. Prime alternative superalgebras of arbitrary characteristic. Algebra and logic, 36:389-420, 1997.

[31] M. F. Smiley. Jordan homomorphisms onto prime rings. Trans. Amer. Math. Soc., 84:426-429, 1957.

[32] Y. Wang. Functional identities in superalgebras. J. Algebra, 382:144-176, 2013.

[33] Y. Wang and Y. Wang. Jordan superhomomorphisms of superalgebras. Comm. Algebra, 42(1):122-129, 2014. 